\title{
Processing of nano-micro copper materials for the production of conductive circuits
}

\section{Bahaa Hanou Abbas}

A thesis submitted in partial fulfilment of requirements of Swansea University for the degree of Doctor of Philosophy in Materials Engineering

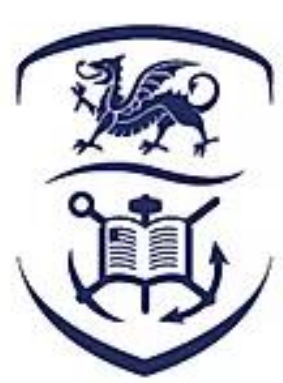

\section{Prifysgol Abertawe Swansea University}

College of Engineering

Swansea University

2020 


\begin{abstract}
Copper inks potentially provide a cost-effective alternative to silver for printed electronic circuits. In glass-based applications such as PV or smart glass, they can provide a means of conductivity enhancement or additional functionality. Three inks consisting of a mixture of nano and micro copper particles were systematically studied to examine the relationship between sintering temperature, sintering time and gaseous environment on the electrical qualities of the sintered printed films deposited on FTO coated glass. There is a definite interaction between the particulate nature of the ink, the sintering conditions, and the conductive properties of the film. Films containing only nano-particles provide the most conductive films with optimum sintering conditions of temperature of $225^{\circ} \mathrm{C}$ for 60 minutes. The inclusion of micro particles increased the ideal sintering temperature but lowered the sintering time. An ink containing an equal mixture of nano and micro particles exhibited the lowest performance. This could be attributed to partial oxidation of the nano-particles along the conductive path, which occurs as a result of the presence of the micro particles. Other samples were photonically sintered using a PulseForge 1200 laboratory photonic sintering unit where the number of pulses, pulse power, pulse frequency and the intra pulse gap could be varied. An initial optimization study identified an operational range of photonic energy profile. The best possible line conductivity obtained using these optimum conditions was around a $1 / 3$ of that obtained by conventional thermal sintering. This relative conductivity of photonically sintered features further deviated from conventionally sintered features as the film thickness increased and as the line width reduced. Laser / NIR techniques were found ineffective to sinter the copper ink used in this study. The possibility to manually blend copper and silver paste ink was investigated and an optimum blend of $25 \%$ silver and $75 \%$ copper could be used which had maintained conductivity, cost, and adhesion benefits.
\end{abstract}




\section{DECLARATIONS}

This work has not previously been accepted in substance for any degree and is not being concurrently submitted in candidature for any degree.

Signed

Date: $01 / 09 / 2021$

This thesis is the result of my own investigations, except where otherwise stated. Other sources are acknowledged by footnotes giving explicit references. A bibliography is appended.

Signed

Date: $01 / 09 / 2021$

I hereby give my consent for my work, if relevant and accepted, to be available for photocopying and for inter-library loans after expiry of a bar on access approved by the University.

Signed

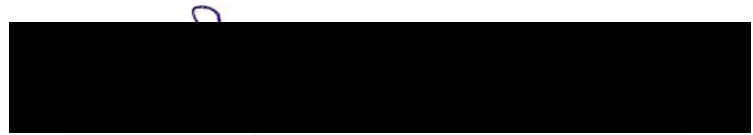

Date: $01 / 09 / 2021$

The University's ethical procedures have been followed and, where appropriate, that ethical approval has been granted.

Signed

Date: $01 / 09 / 2021$ 
I should like to thank Swansea University, Engineering and Physical Sciences Research Council Fund, and Intrinsiq Materials ltd for supplying the materials for the opportunity to study my doctorate at Swansea University. I should also like to thank my academic supervisors, Eifion Jewell, Justin Sealer, and all my colleagues at Swansea University Materials Research Centre for their advice, encouragement and help during various stages of this project. I would also like to thank SPECIFIC team, Youmna Mohamed and Eifion Jewell for their assistance with poking various parties for samples and supplies.

I would also like to thank my wife, ASTA, for the tremendous support during my study. Final thanks go to my brother Alaa Abbas, the best Father Hanou Abbas and my Mother Sabriya Mutalq for their continued support during my university studies. 


\section{Supporting Peer Reviewed Work}

Journal publication, conference presentation and posters relating to this work are listed below.

\section{Publication}

1. B. Abbas, Y. Mohammad, E. Jewell, and J. Searle, "Thermal sintering of printable copper for enhanced conductivity of FTO coated glass substrates," J. Mater. Sci. Mater. Electron., no. 0123456789, pp. 17-19, 2019, doi: 10.1007/s10854-019-02358-x.

\section{Conference Presentation and Posters}

1. Bahaa Abbas, John Lau, Youmna Mohammad, Eifion Jewell, Justin Searle \& Tim Claypole A study of the potential for photonic processing of nano copper Swansea University. innoLAE 2018.

2. Y.Mouhamad, B. Abbas, E. Jewell, J.R. Searle. S. Meroni, F. De Rossi and T.Watson. "Electrode micro patterning for enhanced charge extraction from pv cell", XXVII International Materials Research Congress, Cancun, Mexico, August 21-24 2018. (Conference) 


\section{$\underline{\text { Table of Contents }}$}

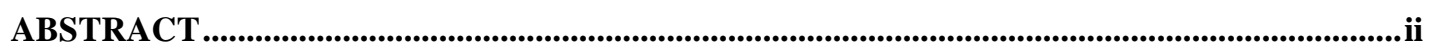

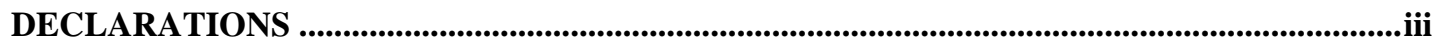

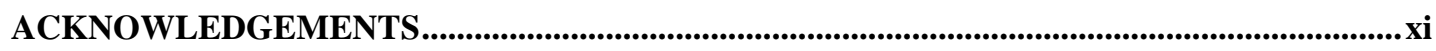

LIST OF FIGURES ................................................................................................................

LIST OF TABLES ............................................................................................................................

ABBREVIATIONS ...............................................................................................................................................

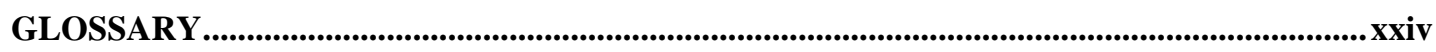

CHAPTER 1: INTRODUCTION ..............................................................................................................

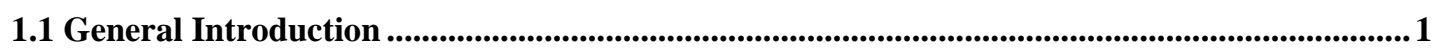

1.2 Aim of the research ...................................................................................................................................

1.3 Literature Review ..................................................................................................................

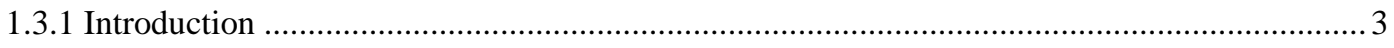

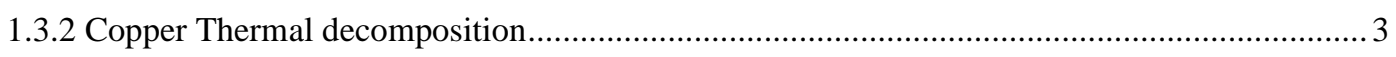

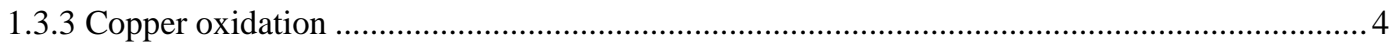

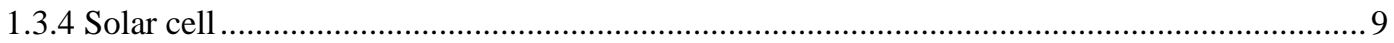

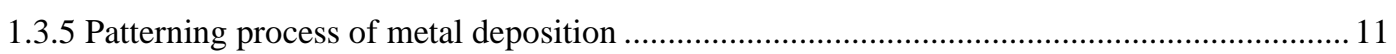

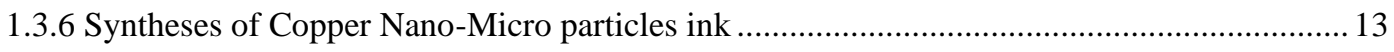

1.3.7 Thermal Sintering of Copper Nano-Micro particles .............................................................. 15

1.3.7.1 Thermal Sintering under nitrogen reducing atmosphere. .............................................. 15

1.3.7.2 Thermal Sintering under Formic Acid atmosphere ....................................................... 17

1.3.7.3 Thermal Sintering under mixed gas atmosphere. ....................................................... 19

1.3.8 Photonic Sintering of Copper Nano-Micro particles ............................................................ 21

1.3.9 Laser Sintering of Copper Nano-Micro particles................................................................ 26

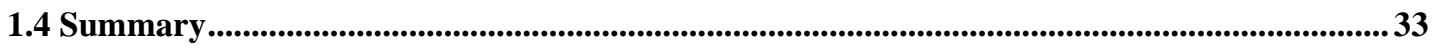

CHAPTER 2. MATERIALS AND EXPERIMENTAL METHODS.................................................34

2.1 Introduction .......................................................................................................................................................34 
2. 2 Materials selection and preparation ............................................................................................34

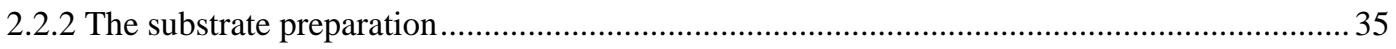

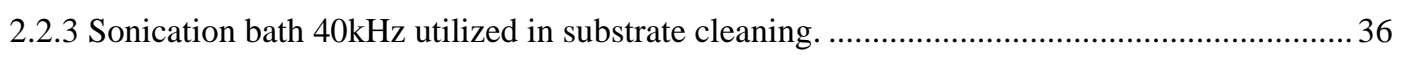

2.2.4: ATMA semi-automatic printer utilized in the screen printing .............................................. 36

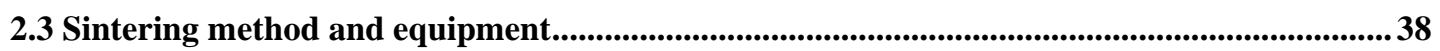

2.3.1 Thermal sintering using Controlled atmosphere oven ......................................................... 38

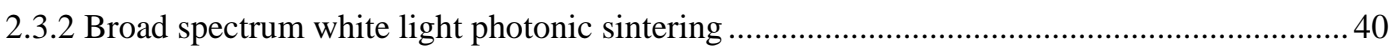

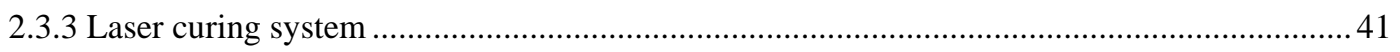

2.3.4 Near infrared (NIR) sintering .................................................................................. 42

2.4. Measurement and Characterization equipment......................................................................43

2.4.1 Brookfield Rheometer ………………………............................................................ 43

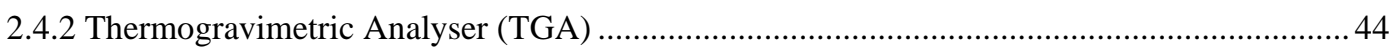

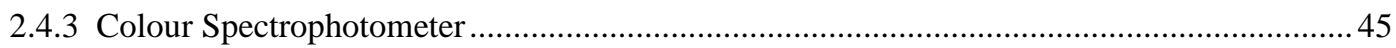

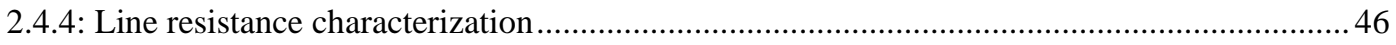

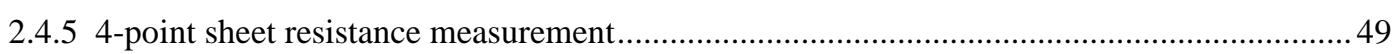

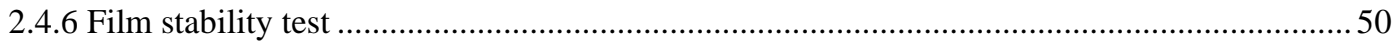

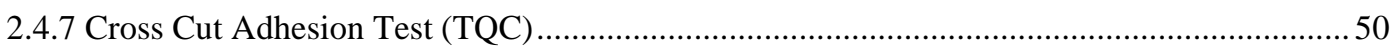

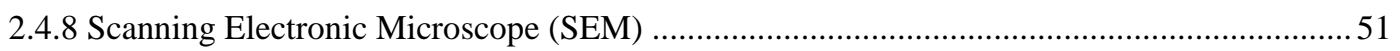

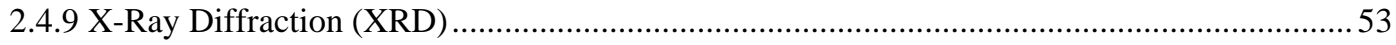

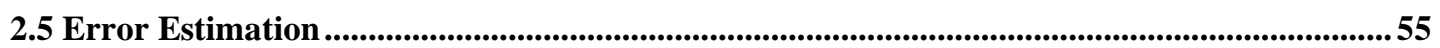

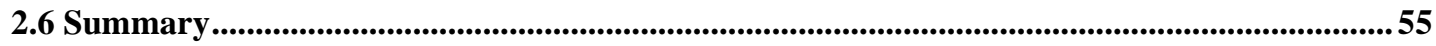

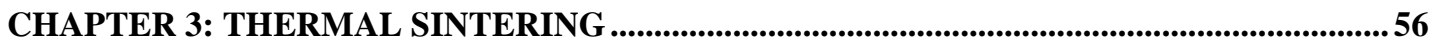

Introduction .............................................................................................................................................................56

3.1 Materials Characterization of the wet ink ..................................................................................56

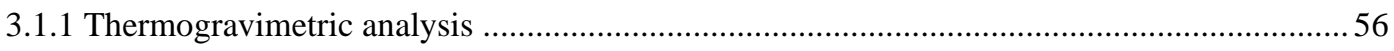

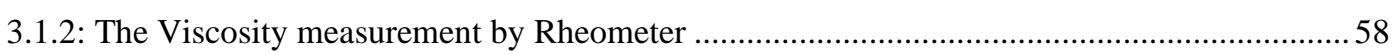

3.2: Material characterization of the dry, un-sintered film ....................................................................59

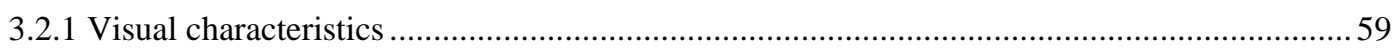

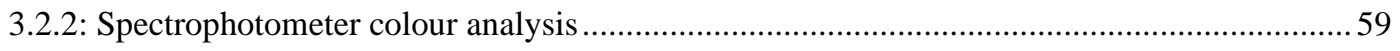

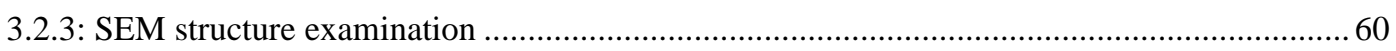

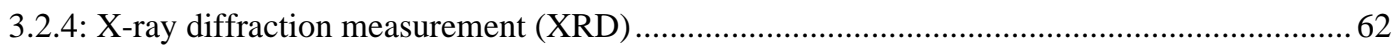

3.3: Thermal sintering under the ambient condition ..............................................................62

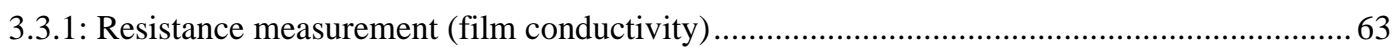

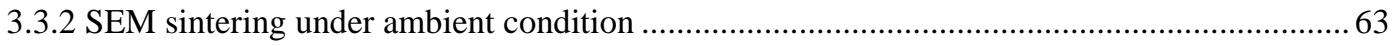


3.3.3: (EDS) examination sintering under the ambient condition.....

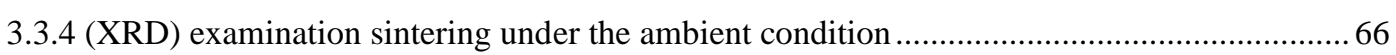

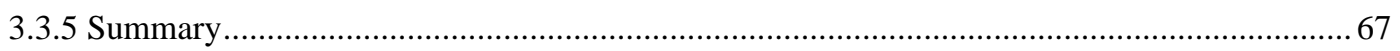

3.4 Thermal sintering under controlled oven atmosphere.....................................................67

3.4.1: Resistance measurement (film conductivity) under controlled atmosphere ........................6 67

3.4.2: Sheet resistance by 4-point probe under controlled atmosphere ........................................ 70

3.4. 3: TLM measurement of sintering under controlled atmosphere ............................................. 72

3.4.4: Adhesion test after thermal sintering under controlled atmosphere .................................... 74

3.4.5: SEM examination sintering under controlled atmosphere ............................................. 75

3.4.5.1: SEM examination Formic acid sintering condition .................................................... 78

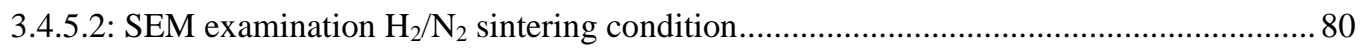

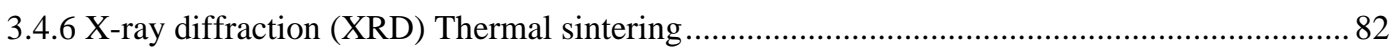

3.4.7: Stability test after Thermal sintering under reducing atmosphere ......................................... 84

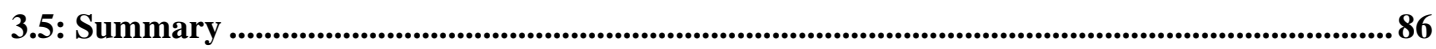

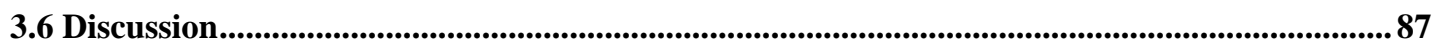

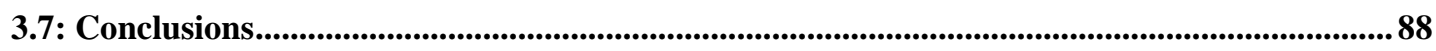

CHAPTER 4: PHOTONIC SINTERING ..............................................................................89

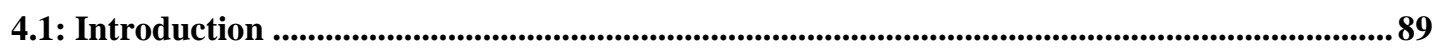

4.2: Optimization the PulseForge Energy ...................................................................................... 89

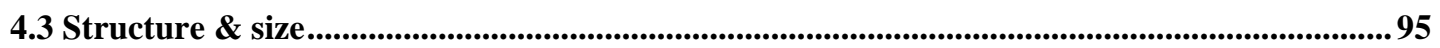

4.4: TLM measurement of Photonically sintered samples.......................................................99

4.5: Sheet resistance by 4-point probe photonically sintered samples ........................................99

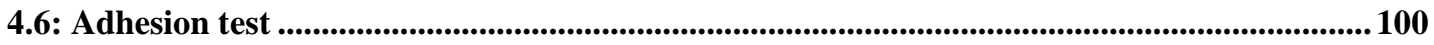

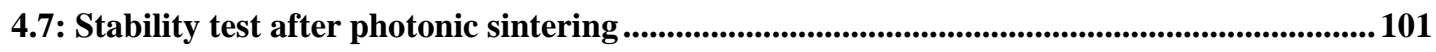

4.8: SEM photonically sintered materials ..................................................................................... 102

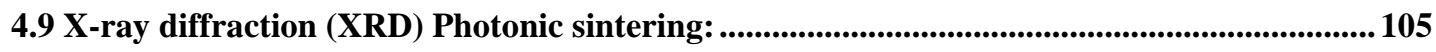

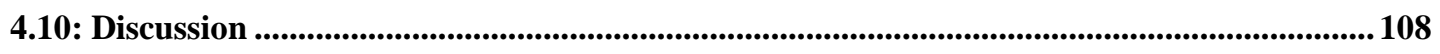

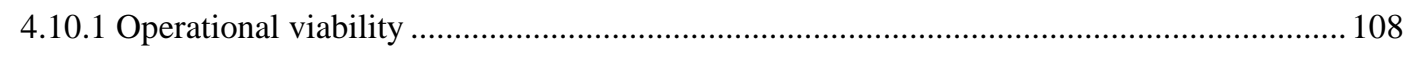

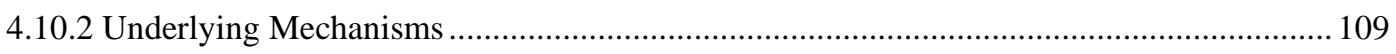


5.1 Introduction

5.2 Optimization of Laser Sintering .

5.3: Near infrared (NIR) sintering.

5.4 Discussion.

5.5: Conclusion

CHAPTER 6: BLENDED CONDUCTIVE MATERIALS

6.1 Introduction

6.2: Optimization of mixed inks

6.3: Resistance measurement (mixed film conductivity)

6.4: Contact resistance of the sintered samples

7.1 Performance of nano vs micro particle inks

7.2 Thermal sintering conditions

7.3 Photonic sintering 
CHAPTER 8: CONCLUSION \& FUTURE WORK

8.1: Future work.

APPENDIX A: ERROR MITIGATION

CHAPTER 9: WORKS CITED .144 


\section{List of Figures}

Figure 1. $1 \mathrm{XRD}$ spectra of thin $\mathrm{Cu}$ films after oxidation in air at different time \& temperatures [32] 6

Figure 1. 2 Copper oxidization mechanism 6

Figure 1. 3 SEM micrographs of a side view of the whiskers formed at the surface ..8

Figure 1. 4 Solar cell pattern grid of the fingers and busbars .9

Figure 1.5 Schematic diagram of solar cell 10

Figure 1. $6 \mathrm{~A}$ schematic cross section of the screen-printing process 11

Figure 1. 7 Schematics of the main type of inkjet head drop on demand piezo electric ink jet

Figure 1. 8 XRD analyses of copper nano-particles stored for (a) 0 month, (b) 3 months [53] 14

Figure 1. 9 SEM (i) $180{ }^{\circ} \mathrm{C}$, (ii) $200{ }^{\circ} \mathrm{C}$, (iii) $230{ }^{\circ} \mathrm{C}$, and (iv) $260{ }^{\circ} \mathrm{C}$ under nitrogen atmosphere for 30min. [65] .16

Figure 1. 10 Resistivity of copper pattern on a polyimide substrate as a function of time sintered at $200^{\circ} \mathrm{C}$ under a reducing atmosphere [72].

Figure 1. 11 The variations of electrical resistivity of copper films under a certain temperature as sintering time increase under $\operatorname{Ar}(96 \%)-\mathrm{H}_{2}(4 \%)[53]$ 20

Figure 1. 12 light generating diagram. 21

Figure 1. 13 XRD of copper nano-ink sintered at different light intensities [82] ......22

Figure 1. 14 Copper resistivity as function of pulse duration (4-15ms). [10]. .23

Figure 1. 15 SEM images of the flashlight irradiated copper nanofilms as changing irradiation energy; (a) $2 \mathrm{~J} / \mathrm{cm}^{2}$, (b) $4 \mathrm{~J} / \mathrm{cm}^{2}$, (c) $6 \mathrm{~J} / \mathrm{cm}^{2}$, (d) $8 \mathrm{~J} / \mathrm{cm}^{2}$, (e) $10 \mathrm{~J} / \mathrm{cm}^{2}$ and (f) $12 \mathrm{~J} / \mathrm{cm}^{2}$. [7]. .24

Figure 1. 16 the skin effect region, the emissivity of copper and silver [84]. .25 
Figure 1. 17 The resistance of laser sintered samples as a function of the optical power of the laser diode. $(50 \mathrm{~mm} / \mathrm{s}, 100, \mathrm{~mm} / \mathrm{s}, 200 \mathrm{~mm} / \mathrm{s}, 400 \mathrm{~mm} / \mathrm{s})$

Figure 1. 18 XRD. (a) As-printed, (b) thermally sintered in ambient air, (c) thermally sintered in $\mathrm{N}_{2}$ atmosphere, (d) laser-sintered in $\mathrm{N}_{2}$ atmosphere[98]. .28

Figure 1. 19 SEM of films sintered at different laser powers of (a) $0.8 \mathrm{~W}$, (b) $1.0 \mathrm{~W}$, and (c) $1.4 \mathrm{~W}[98]$. .29

Figure 1. 20 (a) Sheet resistances plotted as a function of the scan rate and laser power.

Figure 1. 21 The effect of ink drying conditions on sintered $\mathrm{Cu}$ ink film for three different drying levels [99]. .30

Figure 1. 22 Copper line resistivity versus laser irradiance (constant laser scan velocity, $v=3 \mathrm{mms}^{-1}$ ). The insets are bright field microscopy images of the scanned copper line [99]. 31

Figure 1.23 Time dependence of the calculated surface reflectivity (a) and absorption coefficient (b) for a single-shot laser pulse [100] 32

Figure 2. 1 FTO Glass sheet substrate- cut to 36 samples of $50 \times 50 \mathrm{~mm}$ .36 Figure 2. 2 (a): ATMA AT-25PA/AT-45PA Digital Electric Flat Screen Printer, (b): mesh and ink setting.

Figure 2. 3 Screen pattern design. .38

Figure 2. 4 Controlled atmosphere oven .39

Figure 2. 5 (a) photograph of the PulseForge 1200, (b) schematic 40

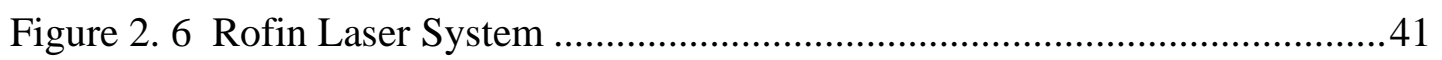

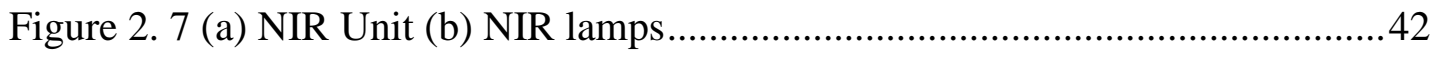

Figure 2. 8 (a) Schematic (b) Photograph of the brookfield rheometer.....................44 
Figure 2. 9 (a) Photograph of the TGA (b) Schematic .45

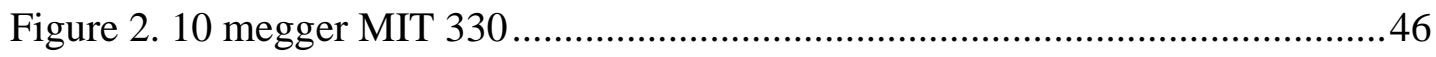

Figure 2. 11 Printed line Diagram.................................................................. 47

Figure 2. 12 Plot to extract transfer length from the TLM method ..........................48

Figure 2. 13: Plot to extract transfer length from the TLM method ..........................48

Figure 2. 14 The 4-points probe sheet resistance measurement ..............................49

Figure 2. 15 Lattice pattern with six cuts and peel profile...................................50

Figure 2. 16 Classification of Adhesion Test (Tape after the test) .........................51

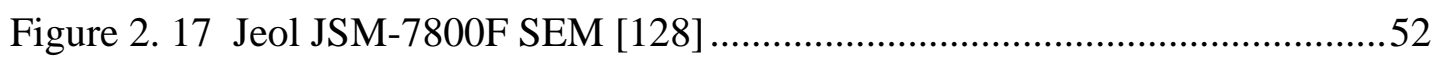

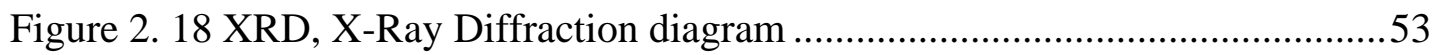

Figure 3. 1 TGA Thermal characteristics .........................................................56

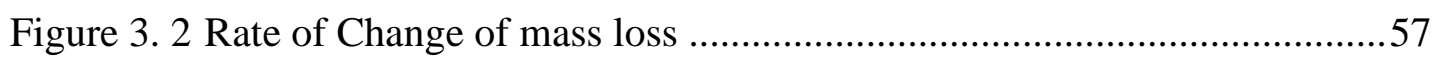

Figure 3. 3 Rheological characteristics of 3 materials over a 0 - 150 shear rate range.

Figure 3. 4 (10X) Dry screen-printed inks at $80^{\circ} \mathrm{C}$ for 30 minutes ..........................59

Figure 3.5 identifying the difference between nano and micro-particles ................60

Figure 3. 6 Dry screen-printed inks at $80^{\circ} \mathrm{C}$ for 30 minutes ..................................61

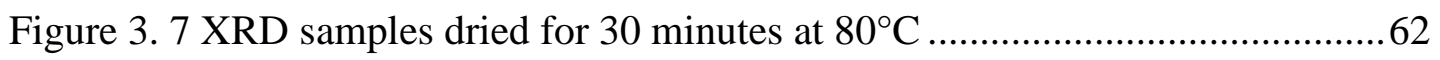

Figure 3. 8 Thermally Sintered samples for 60 mins at $200{ }^{\circ} \mathrm{C}$ in the oven ..............63

Figure 3. 9 SEM of films sintered in air at $200^{\circ} \mathrm{C}$ for 30 minutes ...........................64

Figure 3. 10 EDS: 100N ink sintered under ambient condition..............................65

Figure 3. 11 EDS: 50N50M ink sintered under ambient condition .........................65

Figure 3. 12 EDS: 20N80M ink sintered under ambient condition .........................65

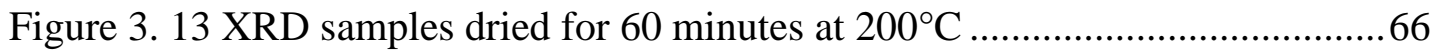


Figure 3. 14 Copper Nanoparticles in the Presence of Capping Agents in Organic Solvent [143]

Figure 3. 15 Line resistance for a nominal $600 \mu \mathrm{m}$ wide line of $30 \mathrm{~mm}$ length under each sintering environment at residence times of 30, 60, 90 and 120 minutes. .69

Figure 3. 16 Sheet resistance of each ink under the $\mathrm{N}_{2}$, Formic acid and $5 \% \mathrm{H}_{2} / \mathrm{N}_{2}$ sintering environements at residence times of 30, 60, 90 and 120 minutes..... .71 Figure 3. 17 Contact resistance of each ink under the N2, Formic acid and $5 \% \mathrm{H}_{2} / \mathrm{N}_{2}$ sintering environements at residence times of 30,60 and 90. .73

Figure 3. 18 Contact resistance under nitrogen atmosphere at $275^{\circ} \mathrm{C}$ for 30 minutes

Figure 3. 19 100N SEM of the dried and nitrogen sintered films. .76

Figure 3. 20 SEM images of the dry and $\mathrm{N}_{2}$ sintered film. .77

Figure 3. 21 SEM images of the dry and formic acidsintered films $100 \mathrm{~N}$ .78

Figure 3. 22 SEM images of the dry and formic sintered films (50N50M and 20N80M) .79

Figure 3. 23 SEM images of the dry and $\mathrm{H}_{2} / \mathrm{N}_{2}$ sintered films $100 \mathrm{~N}$ 80

Figure 3. 24 SEM images of the dry and $\mathrm{H}_{2} / \mathrm{N}_{2}$ sintered films (50N50M and 20N80M) .81

Figure 3. 25 XRD comparison of each film for each ink in a dry, $\mathrm{N}_{2}$, Formic acid and $\mathrm{H}_{2} / \mathrm{N}_{2}$

Figure 3. 26 Line resistance change over 4-week interval (labelled 1-4) for the three materials sintered at 30 minutes in each sintering environment. .85

Figure 3. 27 Charge transfer through the particles. .87

Figure 3. 28 Charge transfer between the electrode and the conductive film. .88 
Figure 4. 1 Energy - pulses relationship ........................................................... 90

Figure 4. 2 Energy - simulated PulseForge data ................................................ 92

Figure 4. 3 Line resistance for the $600 \mu \mathrm{m}$ wide line for each ink under each photonic illumination regime.

Figure 4. 4 Examples where the degree of sintering differs between adjacent features at different geometric sizes. .95

Figure 4. 5 Normalised resistance as a function of energy for each ink and line width.

Figure 4. 6 Resistivity under photonic sintering condition .97

Figure 4. 7 Contact resistsiance for the photonically sintered samples.

Figure 4. $8100 \mathrm{~N}, 50 \mathrm{~N} 50 \mathrm{M}$ and 20N80M ink, sheet resistance photonically sintered with different energy setting 100

Figure 4. 9 Line resistance change over time in weeks for the three materials sintered 101

Figure 4. 10 SEM images of the $100 \mathrm{~N}$ ink ( $\mathrm{a}$ and $\mathrm{b}$ ) thermally sintered (formic acid) and (c and d) photonically sintered films 102

Figure 4. $11 \mathrm{FTO}-100 \mathrm{~N}$ Interface when photonically sintered 103

Figure 4. 12 SEM images of the thermally and photonically sintered films for the 50N50M ink. 104

Figure 4. 13 SEM images of the Formic and photonically sintered films for the 50N50M ink. 105

Figure 4. 14 XRD of the 100N, 50N50M ink -copper patterns $(600 \mu \mathrm{m}$ line width) 106

Figure 4. 15 XRD 20N80M ink $(600 \mu \mathrm{m}$ line width $)$ 107 
Figure 5. 1: SEM images of Photonic sintering vs laser sintering for laser samples

4,5 and 6 115

Figure 5. 2: SEM images of Dry only vs NIR sintering at $50 \% \& 40 \mathrm{~s}$. 120

Figure 6. 1: Mixed ink (silver-copper)

Figure 6. 2: The impact of silver content on the resistance of the 600-micron line 125 Figure 6. 3: Mixed ink line resistance $\mathrm{H}_{2} / \mathrm{N}_{2}$ condition compare to $\mathrm{Ag}$ and $\mathrm{Cu} \ldots . . .125$ Figure 6. 4: Mixed ink absolute resistivity sintered under $\mathrm{H}_{2} / \mathrm{N}_{2}$ condition compare to silver nano-particle resistivity 126 Figure 6. 5: Mixed ink contact resistance $\mathrm{H}_{2} / \mathrm{N}_{2}$ sintered related to pure silver and 20N80M copper.

Figure 6. 6: Mixed ink, sheet resistance $\mathrm{H}_{2} / \mathrm{N}_{2}$ sintered related to pure silver and 20N80M copper 128

Figure 6. 7 .SEM Nano-silver, 20N80M copper with the mixed ink of Ag 25\% .... 130 Figure 6. 8: SEM Nano-silver, 20N80M copper ink blended with (a) 25\%, (b) 54\% and (c) $75 \% \mathrm{Ag}$ 131

Figure 6. 9 EDS: Ag ink sintered under ambient condition. 132

Figure 6. 10: EDS of $\mathrm{Ag} \% 25 \mathrm{ink}$ sintered under $\mathrm{H}_{2} / \mathrm{N}_{2}$ condition 132

Figure 6. 11: EDS of $\mathrm{Ag} \% 54$ ink sintered under $\mathrm{H}_{2} / \mathrm{N}_{2}$ condition 133

Figure 6. 12: EDS of $\mathrm{Ag} \% 75$ ink sintered under $\mathrm{H}_{2} / \mathrm{N}_{2}$ condition 133

Figure 6. 13: XRD response of each mixed metal ink before (dry) and after the sintering process for 30 mins in a $\mathrm{H}_{2} / \mathrm{N}_{2}$ environment for $250{ }^{\circ} \mathrm{C}$. 134 
Figure 6. 14: XRD comparison of each film for each film sintered at $5 \% \mathrm{H}_{2} / \mathrm{N}_{2}$ for 30 min @ $250{ }^{\circ} \mathrm{C}$ in comparison to the unmixed material........................................ 135 


\section{List of Tables}

Table 1. 1 The summary of sample identification (1D) and pulse conditions to the total

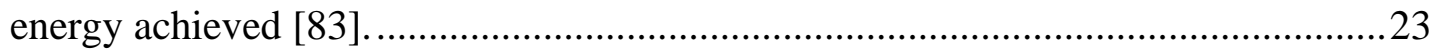

Table 1. 2 Optical properties of undoped and V-doped copper oxide thin films [85] .

Table 2. 1 Copper inks examined in the study (visually sized by SEM) .34

Table 2. 2 The print setting .36

Table 3. 1 (L*a*b* absolute Colour) .59

Table 3. 2 (Adhesion tape test result after thermal sintering) .74

Table 3. 3 Optimum conditions and resultant sheet resistance and relative resistivity for each ink and atmosphere (Relative resistivity to copper at $\rho_{0}=1.68 \times 10^{-8} \Omega \mathrm{m}$ ). 86

Table 4. 1 Energy - simulated PulseForge data... .92

Table 4. 2 Absolute resistivity relationship for each material for the 600um line..... 98

Table 4. 3 Optimum sintering conditions for each material for the 600um line .98

Table 4. 4 Adhesion tape test result 100

Table 5. 1 Laser settings used in the investigation at wavelength of $532 \mathrm{~nm}$. 113

Table 5. 2: NIR power used in the investigation (20N80M ink) 117

Table 6. 1 Mixed ink ratio.

Table 6. 2: Absolute resistivity relationship for each material for the 500 $\mu \mathrm{m}$ line 126 Table 6. 3: Adhesion tape test results for each mixed metal ink sintered at $250^{\circ} \mathrm{C}$ for 30 minutes. 129 


\section{Abbreviations}

$100 \mathrm{~N}$

20N80M

100\% Nano-particles

20\% Nano-particles 80\% Micro-

particles

50N50M

50\% Nano-particles 50\% Micro-

particles

$\mathrm{Cu}_{2} \mathrm{O}$

Copper (I) oxide

$\mathrm{CuO}$

Copper (II) oxide

$\mathrm{CuSO}_{4}$

Copper (II) sulphate

CVD

Chemical vapour deposition

$\mathrm{H}_{2} / \mathrm{N}_{2}$

5\% Hydrogen: 95\% Nitrogen

EDX

$\mathrm{eV}$

FTO

$\mathrm{HCHO}$

LT

$\mathrm{MPa}$

NIR

PCB

PVP

Rc

Rs

$\mathrm{Rt}$

Energy-dispersive X-ray spectroscopy

Electron volt

Fluorine- doped Tin Oxide

Formaldehyde

Transfer length

Megapascal Pressure Unit

Near infrared

Printed circuit board

Poly (N-vinyl-2-pyrrolidone)

Contact resistance

Sheet resistance

Total Resistance

SEM

Scanning Electron Microscopy

SP

TGA

Sphere spectrophotometer

Thermogravimetric Analyser

TLM

Transmission line measurement

XRD

X-ray Diffraction 


\section{Glossary}

CP007- Commercial name for $100 \%$ nano copper ink

IMCE4101- Commercial name for 50\% nano/50\% micro copper ink

IMCE4118- Commercial name for $20 \%$ nano/80\% micro copper ink

Intrinsiq - Commercial name for the copper ink supplier

NovaCentrix-Commercial name for the provider of the photonic sintering system

ORGACON- - Commercial name for the nano-silver ink

PulseForge 1200 - Commercial name for the photonic sintering system 


\section{Chapter 1: Introduction}

\subsection{General Introduction}

Copper is the material of choice for circuit boards manufactured using subtractive processes due to its conductivity and low cost [1]. For circuitry manufactured using additive technology, silver is the material of choice as the dispersion of metallic particles is readily manufactured, but copper particles are easily oxidized to their nonconducting oxide [2], [3]. In the last decades, the synthesis of copper nano-particles ink has attracted research attention in manufacturing conductive circuits, because replacing silver nano-particles ink potentially leads to lower cost products.

The conductive circuit is the fundamental part of any electrical or electronic device, and while silver has excellent conductivity, is stable in organic suspensions and has a good lifetime, it is expensive and subject to fluctuations in price. Numerous applications for copper ink have already been proven, and these include radiofrequency identification aerials [4], hybrid silicon/printed flexible circuits [5] and thin film transistor electrodes [6]. Each of these applications focuses on polymer substrates, most notably polyimide because of its ability to withstand higher sintering temperatures, product flexibility and subsequent compatibility with reel to reel processes [7].

Formulation of copper inks can be classified into four main groups: thermal decomposition of soluble copper salts [8], copper oxide/hydroxide nano-particles which are chemically/thermally reduced to copper [9], copper nano/micro-particles which can be sintered and hybrid inks which use a combination of these technologies [10]. The main challenge with using copper nano-particles is oxidation during sintering, as nano-copper is readily oxidized at temperatures over $130{ }^{\circ} \mathrm{C}$ [11]. Proprietary copper inks overcome this problem with an organic coating (capping agent) such as carboxylic acid, or PVP [12], [13], which protects the reactive copper during suspension, deposition and drying, but undergoes decomposition and sublimation during the sintering process creating a conducting copper network of linked particles [14]. 
The particles are linked by copper-copper interfaces which are less susceptible to oxidation because of their lower surface area to volume ratio. Although nano-particles are preferred for their lower sintering temperatures [15], they have an appreciable cost penalty associated with manufacturing in controlled argon atmospheric conditions and filtering. There is, therefore, an economic driver for the move to mixed micro/nano copper systems.

Photonic sintering has proven popular due to its rapid processing speed. Laser sintering has been proven as has broad-spectrum intense white light photonic sintering [16]. Multi-spectrum photonic sintering (IR and white light) [17], [18] also allows for rapid sintering under ambient atmospheric conditions on flexible substrates. All these are highly sensitive to the circuit pattern, film thickness, ink composition, substrate, and other physical and optical properties. Substrates with high thermal mass act as a thermal sink [19], retarding the rapid sintering process, and are particularly problematic and are less suited to photonic sintering.

Most copper printing applications have focused on polymer substrates [20]. However, in glass-based applications such as photovoltaics (PV) or smart glass, it can provide a means of conductivity enhancement or additional functionality [21]. Fluorine doped tin oxide (FTO) coated glass offers a low-cost alternative transparent conductive substrate to indium tin oxide (ITO) coated glass and has been widely adopted by the third generation PV community for organic and perovskite (particularly carbon rear electrode) technologies [22]. These PV technologies have transitioned to the module scale where larger dimensions demand enhanced conductivity of transparent electrodes by printing fine electrode structures between higher transparency and low conductivity FTO for more efficient charge extraction [23]. This concept currently proposes silver but the deposition of printed copper inks for the bus bars and fine charge extraction electrodes within the structure would further reduce the bill of materials through silver ink elimination and thinner and more transparent FTO [24]. 


\subsection{Aim of the research}

The main aim of the study is to evaluate the performance of copper inks printed to FTO conductive glass substrates. Specifically, it aims to identify and understand the relationship and interactions between the final electrical performance of printed film and copper ink characteristics, sintering time, sintering temperature and the atmosphere required for sintering. The findings will help identify processing productivity and capital, and safety requirements in the manufacture of transparent electrodes.

\subsection{Literature Review}

\subsubsection{Introduction}

Throughout this literature review, several subjects need to be observed. The main areas to review are the materials commonly used within printed circuit board manufacturing and the previous work which has been performed in this field. Subsequently, copper forms and how they are transferred from a liquid to a solid state, and nano-particles and thermal degradation of its salts are studied as well as the solar cell screen printing process and possible processes for depositing copper in a pattern. A literature review of copper deposition, plating, and ovens with reducing atmosphere, photonic and other fast sintering processes is undertaken. The challenge during sintering, which is mainly the copper oxidation [25], will be reviewed. From there, potential sintering mechanisms will be evaluated, and appropriate test methods identified. Initially, thermal decomposition and copper oxidation are essential processes to understand the challenges and the behaviour of the material.

\subsubsection{Copper Thermal decomposition}

Thermal decomposition can be simply defined as a chemical decomposition reaction caused by heat [26]. The metal carbonates such as copper carbonate can break down when high temperature directly applied to it. Equation 1.3 illustrates the thermal decomposition for copper carbonate.

$$
\mathrm{CuCO}_{3} \stackrel{\Delta}{\rightarrow} \mathrm{CuO}+\mathrm{CO}_{2} \quad \mathrm{Eq} \ldots 1.1
$$


Applying heat energy causes one compound to break into two or more substances and can simply be explained as the heat breaking down the bond between the atoms of the original molecule. However, thermal reaction considers as high energy consumption which is less demandable compared to renewable energy resources.

There are two main types of thermal decomposing in the gas phase and the solid phase. The thermal decomposition in the solid phase is commonly used in the preparation of nano-particles. There are a few parameters can affect the development of the coppernano-particles such as reaction time which can affect particle size [27]. The temperature required and the amount of solvent used also can be reduced when smaller particles prepared [28]. However, several factors can also impact the thermal decomposition process of copper in organic material[29] such as solvent, surfactant and reducing agent and considering the concentration of these materials.

In 2018 Fukuda studied the mechanisms of thermal decomposition of copper (II) hydroxide as a possible method to produce $\mathrm{CuO}$ nano-particles. The kinetic demonstrating of the decomposition reaction examined via the kinetic analysis of the mass-loss at different temperatures $\left(30^{\circ} \mathrm{C}-50^{\circ} \mathrm{C}\right)$. The key finding of this study is that the mass loss linearly increasing with temperature applied [30]. The crystallite size of the obtained $\mathrm{CuO}$ on nano-particles range and the specific surface area increased steadily. Further heating used led to the crystal growth size of $\mathrm{CuO}$. Therefore, the most efficient reaction stage to obtain $\mathrm{CuO}$ nano-particles achievable was with lower temperature.

\subsubsection{Copper oxidation}

Copper oxidation can be described as when copper is exposed to air, will be oxidized due to a reaction with oxygen. This takes place when the copper atom initially reacts with oxygen molecules. The first primary reaction of copper with the oxygen in the ambient atmosphere will convert copper to copper (I) oxide, as shown in Equation 1.2. This conversion of copper to copper (I) oxide is followed by further oxidation to form $\mathrm{CuO}$, as shown in Equation 1.3.

$$
\begin{aligned}
& 4 \mathrm{Cu}+\mathrm{O}_{2} \rightarrow 2 \mathrm{Cu}_{2} \mathrm{O} \\
& 2 \mathrm{Cu}_{2} \mathrm{O}+\mathrm{O}_{2} \rightarrow 4 \mathrm{CuO}
\end{aligned}
$$


As copper can easily be oxidized in the ambient atmosphere, this is considered as a significant challenge in the solar cell industrial applications. The reduction can be achieved by providing an additive. This is one of the solutions to overcome this challenge. The reduction process can be chemically defined as a chemical reaction in which hydrogen is added or oxygen is removed from a compound. It is also the process used to convert metal oxide into metal [31].

Nowadays, the noble nano-particles inks such as silver are widely utilized in solar cell manufacturing because of their stability and the excellent conductivity they provide [21]. However, the main reason for their popularity is the sintering under the ambient condition in contrast to copper. However, the copper nano-particles printed ink cannot be sintered thermally in the ambient condition. A potential solution for this major issue has been investigated by many researchers around the world using a variety of sintering methods.

The oxidation mechanism in a thin copper film was investigated by Choudhary et al. [32]. Thin copper films with various thicknesses were deposited on glass and silicon substrates by employing the vacuum thermal evaporation deposition process. This research specifies that nanostructured thin films of $\mathrm{Cu}_{2} \mathrm{O}$ and $\mathrm{CuO}$ can easily be reached with the simple thermal method in the ambient condition. X-ray diffraction (XRD) examination shows the formation of highly crystalline films of copper oxides at different times and temperatures (Figure 1.1). The study also suggests that oxidation of the thin copper film is highly sensitive to the diffusion kinetics, which can be related to the film particle sizes [33]. However, after thermal exposure for $25 \mathrm{~h}$, the final oxide state of the thin copper film was found to be correlated with the temperature applied. The thin copper film was initially oxidized to form $\mathrm{Cu}_{2} \mathrm{O}$ when thermally treated at a temperature above $150^{\circ} \mathrm{C}$, which is considered as a p-type semiconductor [34]. Further curing at a temperature above $330^{\circ} \mathrm{C}$ in an ambient condition led to the formation of a $\mathrm{CuO}$ layer on the previously obtained $\mathrm{Cu}_{2} \mathrm{O}$ film. 

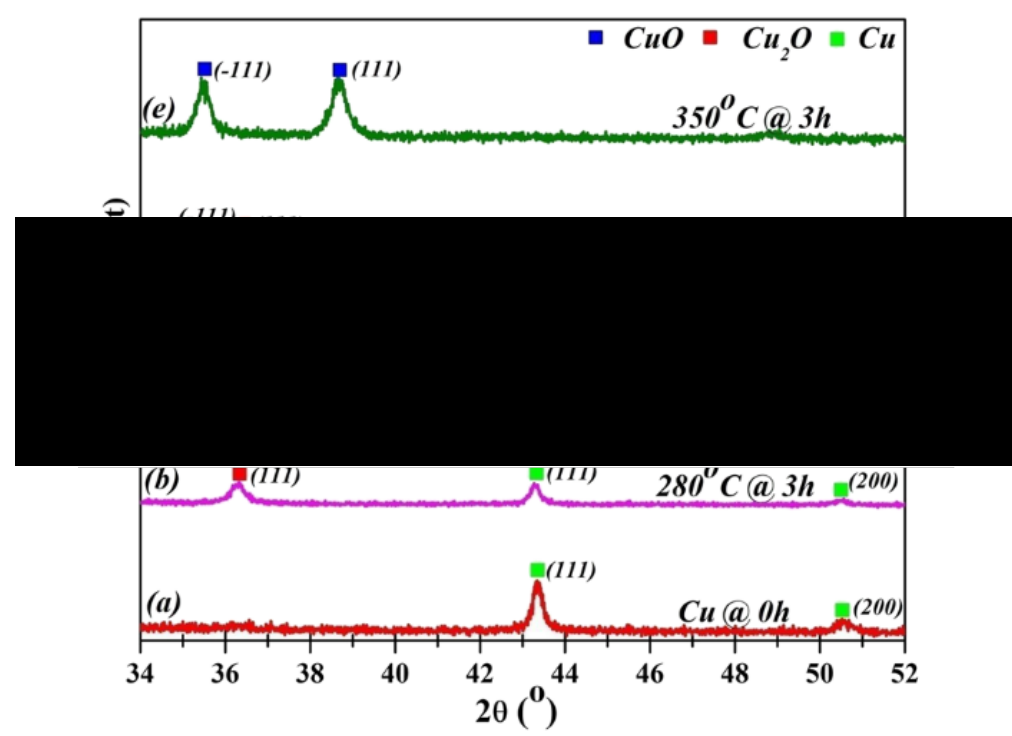

Figure 1.1 XRD spectra of thin $\mathrm{Cu}$ films after oxidation in air at different times and temperatures [32]

A simple illustration of the copper oxidization mechanism is presented in Figure 1.2, which shows a schematic of the surface oxidation steps of thin copper films and the effect of temperature on the oxidation levels.

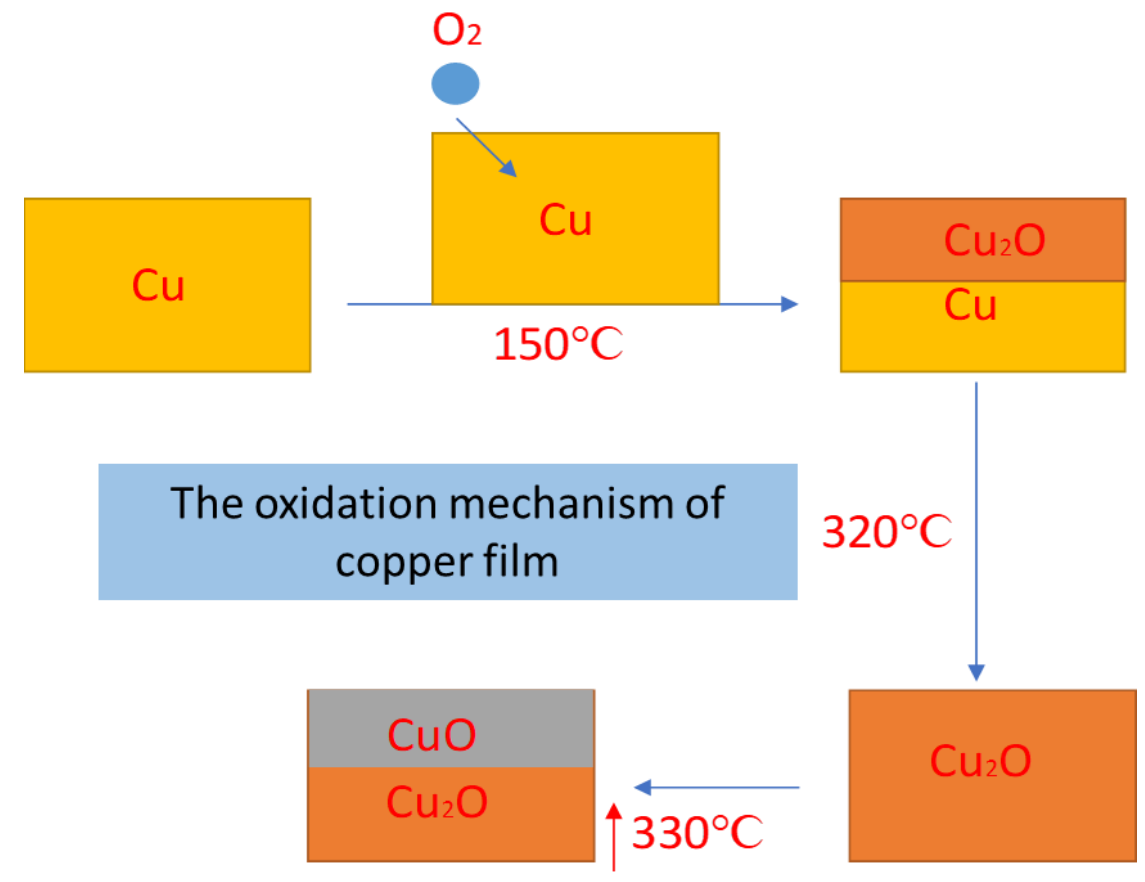

Figure 1.2 Copper oxidization mechanism 
Moreover, in 2002, Zhu et al. investigated the copper oxidation mechanism at a high temperature $\left(350^{\circ} \mathrm{C}-1050^{\circ} \mathrm{C}\right)$; the oxidation process was carried out under $0.1 \mathrm{MPa}$ oxygen. Several studies showed that above $600^{\circ} \mathrm{C}$, the thin $\mathrm{CuO}$ film is secure and impervious to oxygen, and the oxygen potential at the $\mathrm{Cu}_{2} \mathrm{O} / \mathrm{CuO}$ interface is determined by the dissociation pressure of $\mathrm{CuO}$, which is constant at high temperature. The results suggested that the oxidation kinetics of copper is dependent on the ambient oxygen pressure in this temperature range. The amounts of impurities also have a significant effect on copper oxidation, as high purity means less oxidation [35].

Several studies have reported that the temperature is the main parameter in copper oxidation. Lee et al. studied the copper oxidation behaviour with a range of low temperatures between $200^{\circ} \mathrm{C}$ and $300^{\circ} \mathrm{C}$. They defined the oxide layer as three areas: a thin $\mathrm{CuO}$ layer, a thick $\mathrm{Cu}_{2} \mathrm{O}$ layer, and an oxygen-containing area. The main finding was that oxidation at any temperature below $300^{\circ} \mathrm{C}$ can be controlled by diffusion around the boundaries of the $\mathrm{Cu}_{2} \mathrm{O}$ particles. Increasing the size of the $\mathrm{Cu}_{2} \mathrm{O}$ crystals in the surface oxide will reduce the paths for mass transportation. However, the study concluded that the oxidation rate could be reduced by producing larger particle sizes of $\mathrm{Cu}_{2} \mathrm{O}$. Accordingly, pre-oxidation treatment was required by applying a high temperature of $600^{\circ} \mathrm{C}$. This treatment was applied to produce large $\mathrm{Cu}_{2} \mathrm{O}$ crystals in the surface oxide; subsequently, the oxidation was significantly reduced at a temperature below $300^{\circ} \mathrm{C}$ [36].

Haugsrud investigated the water vapour effect on copper oxidation at the University of Oslo in 2002. A different oxygen pressure and water vapour were applied to oxidize the high purity copper used in this study, with a complex flowmeter controlling the gas mixer. The gas mixture was divided into two parts; the first part was dried over $\mathrm{P}_{2} \mathrm{O}_{5}$ ( Phosphorus pentoxide) leaving three ppm of $\mathrm{H}_{2} \mathrm{O}$ in the gas, and the other part was fed through the saturated $\mathrm{KBr}$ solution giving a water vapour pressure of $0.02 \mathrm{~atm}$ at room temperature. The oxidation rate of high purity copper was measured as a function of the oxygen pressure and water vapour pressure at $500^{\circ} \mathrm{C}$ and as a function of temperature in dry and wet air in the range $400^{\circ} \mathrm{C}-1000^{\circ} \mathrm{C}$. The scanning electron microscope (SEM) image in Figure 1.3 shows that the oxide whiskers were covering the surface, and the amount of these whiskers increased when the water vapour and the oxygen pressure were increased at $500^{\circ} \mathrm{C}$. The study concluded that the effect of 
water vapour at room temperature on copper oxidation depends on the grain boundary effect and cannot be relied on, as the researcher suggested; therefore, further studies are required to observe this effect fully [37].

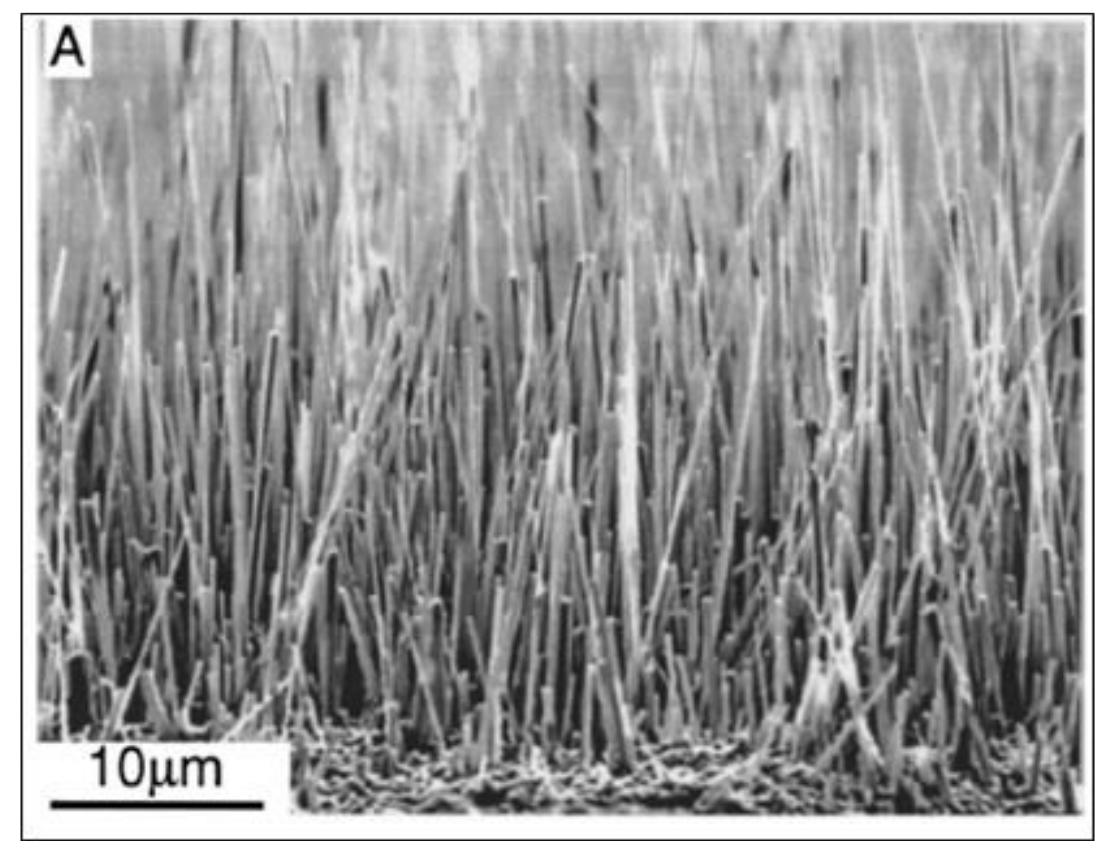

Figure 1.3 SEM micrographs of a side view of the whiskers formed at the surface

$\mathrm{CuO}$ ) and $\mathrm{Cu}_{2} \mathrm{O}$ in nano-crystals have also been investigated by several researchers due to the high potential of using them in high-temperature semiconductor and solar cell applications. Yen et al. investigated the optical properties of the nano-crystals as a function of size. The monodisperse stable $\mathrm{Cu}_{2} \mathrm{O}$ nano-crystals were synthesized by using a novel wet chemistry method. This reliable method have an advantage of nanocrystals and narrow size distributions on copper nano-crystal stability [38].

Generally, in solar cell manufacturing, the front contact consists of a pattern grid comprising the fingers and busbars. Silver paste is the most common material used to form these grids deposited by screen printing [39]. The solar cell is reviewed in the next section. 


\subsubsection{Solar cell}

A solar cell is a viable way to convert the sun's rays directly into electricity that can be utilized. The active part of a solar cell is a wafer made of a semiconductive material (typically silicon), which can be made more conductive via a process called doping. Doping can be defined as the process of intentionally adding impurities to a semiconductor to change its electrical properties [40]. The semiconductor part of the solar cell has three layers. The thin top layer, also known as the negative-type or ntype, contains silicon doped with phosphorus, which has more electrons than silicon. This gives the top layer access to electrons that are free to move and makes the material more conductive. The bottom layer, also known as the positive-type or p-type, contains silicon doped with boron, which has fewer electrons than silicon. This gives the bottom layer fewer electrons that are free to move, thereby making the material less conductive. A missing electron or the hole can be described as an effective positive charge. The thicker middle layer has only slightly fewer electrons, which makes it marginally a p-type [41].

The final step in standard solar cell manufacturing is the formation of a conductive grid pattern comprising fingers and busbars to collect the light-generated electrons. This study focuses on the thick metal lines that are printed on the top n-type layer (typically made of silver). Figure 1.4 shows the printed pattern of fingers and busbars. The bottom p-type layer is in contact with the aluminium plate.

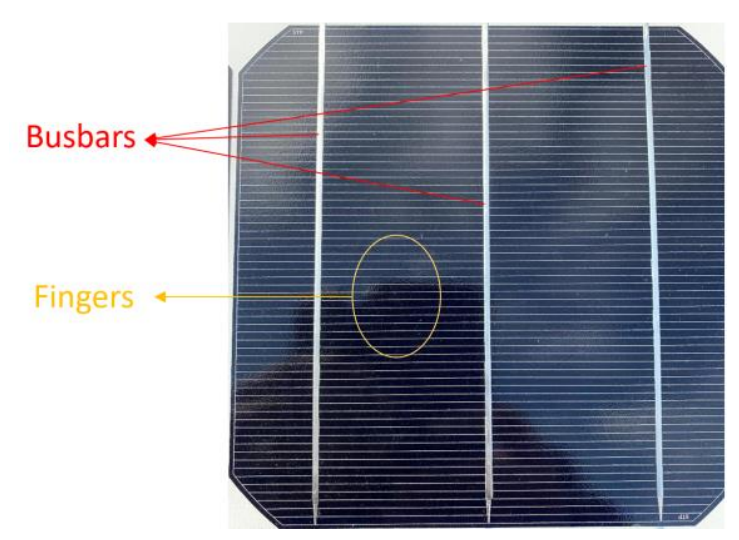

Figure 1.4 Solar cell pattern grid of the fingers and busbars 
The description of the solar cell structure helps to explain how a solar cell generates electricity. In simple terms, the sun emits energy in the form of waves. These waves can range in length, from the short ultraviolet waves through the rainbow of the visible spectrum to long infrared waves. When the sunlight hits the solar cell's top surface, only light with a wavelength of $350 \mathrm{~nm}-1140 \mathrm{~nm}$ is absorbed into the middle layer of the solar cell. This range of wavelengths includes the visible spectrum. Ultraviolet wavelengths are so short so that they stop at the surface. On the other hand, infrared wavelengths are so long that they cannot be absorbed and pass through the cell or are reflected if FTO glass is used.

The light wave knocks an electron off the silicon atom in the middle layer, setting the electron free and leaving an area of positive charge (a hole). The free electron then moves towards the top and reaches the top n-type layer, which readily accepts electrons. Similarly, the free hole moves towards the bottom and reaches the p-type layer, which readily accepts holes. This process continues as long as the sunlight shines on the solar cell.

The electrons and holes are now separated, linking a wire between the top electrode made from printed silver, and the bottom aluminium electrode provides a pathway for the electrons to move towards the holes. This can be used as a source of electricity. The solar cell structure is illustrated in Figure 1.5.

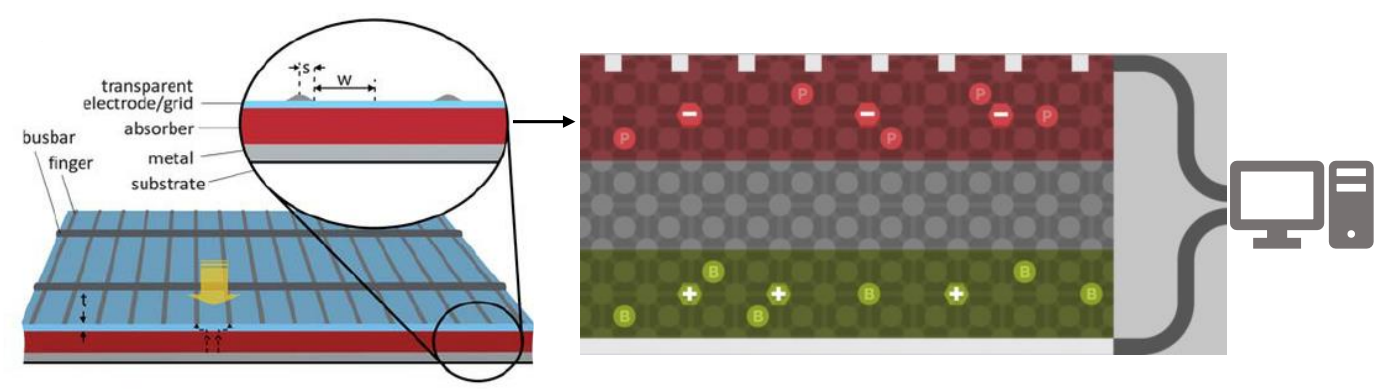

Figure 1.5 Schematic diagram of solar cell 


\subsubsection{Patterning process of metal deposition}

The most common processes for depositing copper in a pattern are screen printing and inkjet printing [42] [39]. Pattern printing can be used to produce sensors, flexible connectors, single- and double-sided PCBs, touch panel bezel contacts, conductive fabrics, batteries, and solar cells.

Screen printing is the deposition and patterning method that is being used, as it provides a method by which a liquid paste can be placed on a substrate such as glass, silicon, and epoxy. It offers the ability to deposit a thin or thick film with a wide rheological window with minimum pressure over a wide area at reasonable production rates [43]. The screen printing of copper ink is the method of choice for a wide range of applications, such as PCB resistors and smart glass [21] or carbon in production of resistive heating elements [44]. Many of these applications present substantial challenges for the material formulator and sintering process. The principle of screen printing is shown in Figure 1.6. A screen holds the pattern image while the ink is held on the top side of the screen.

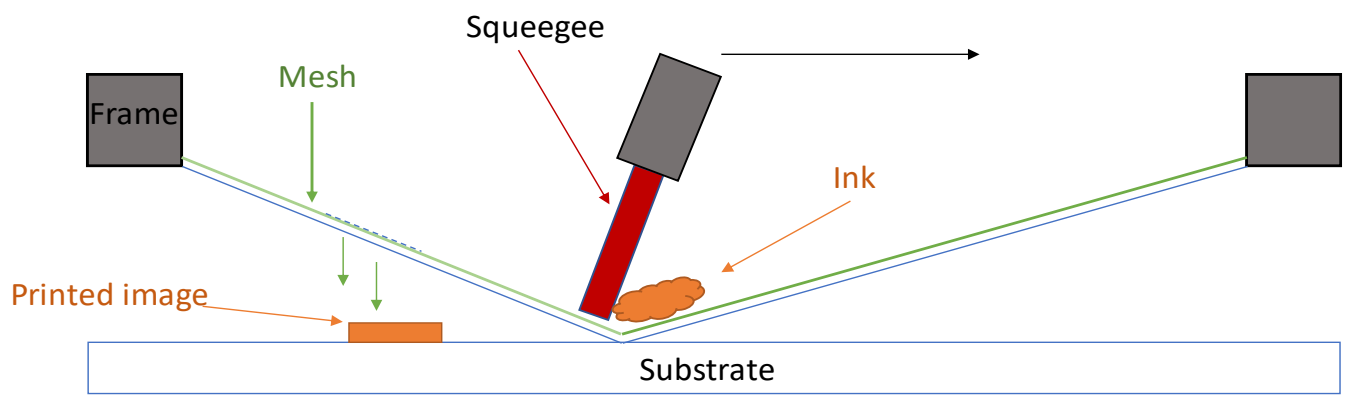

Figure 1.6 A schematic cross-section of the screen printing process

The passage of the squeegee over the screen pushes the ink through the screen to the substrate. Principally, a screen-printing system depends on four distinctive elements: press, screen, squeegee, and flowcoat and ink. 
Inkjet printing is an efficient method for direct pattern deposition of materials on flexible substrates and a fragile substrate used in the production of electronic devices, sensors, and light-emitting diodes [45]. Inkjet printing is one of the most challenging deposition processes; this is attributed to the material formulation. The inkjet printing process is highly dependent on the rheological behaviour and which must be matched precisely to the used inkjet head. An ink made for one head may not operate in another head, and even a small change in the ink properties could require a change in the head or its operational parameters.

Figure 1.7 shows a schematic of the main type of inkjet head drop on demand piezoelectric inkjet.

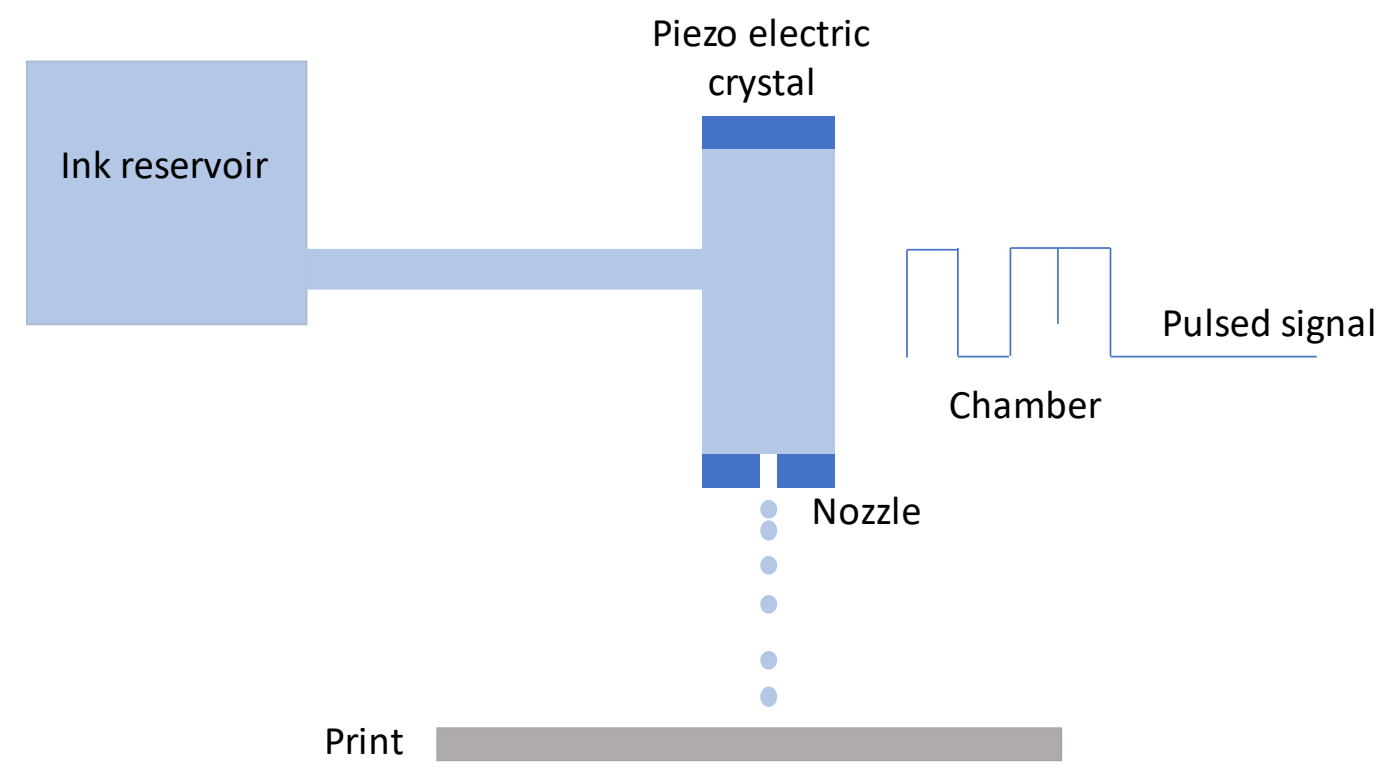

Figure 1.7 Schematic of the main type of inkjet head drop on demand piezoelectric inkjet

As a result of these challenges with inkjet printing, screen printing can be considered as a more suitable procedure to obtain a copper pattern using copper nano-particles ink, as it has a wider operation window in terms of formulation of materials, which is reviewed in section 1.3. 


\subsubsection{Syntheses of copper nano- and micro-particles ink}

Metallic nano-particles are elementary particles with dimensions of less than $100 \mathrm{~nm}$. As this work focuses on using copper nano- and micro-particles ink in the production of a conductive film, it is essential to comprehend how they are prepared, as well as the materials used in its production. There are several chemical methods to make copper paste. However, in this section, only the general method is described.

In simple terms, copper (II) sulphate pentahydrate, as a precursor salt, is the primary chemical material in the preparation of copper nano-particles ink [46]. First, extracting copper sulphate from a pure solid copper wire requires several steps to achieve the electrochemical reaction. The first is immersing two copper wires in sulphuric acid solution, and the second is connecting the wires to a source of electricity, for example, a battery. Subsequently, when the power runs through copper electrodes (anode and cathode) in a dilute sulphuric acid bath, the cathode will produce bubbles of hydrogen gas while the anode will release copper ions into the sulphuric acid and they will be oxidized by the current. Some of the copper from the anode will make its way to the cathode, where it will be reduced. The electrochemical bath that turns a blue colour contains the copper sulphate, which will then become a blue powder [47] [48]. There is an alternative method to the electrochemical process to make copper sulphate by dissolving copper oxide in sulphuric acid [49]. Copper nano-particles can be prepared by adding, for example, ethylene glycol as a solvent to copper sulphate and propylene glycol as a polymeric electrically conductive binder. A copper paste will form copper nano-particles after drying and being fastened with these polymers. A homogeneous film can be achieved from it, as the polymers used must have good electrical conductivity [50].

Blosi et al. investigated the use of a microwave in synthesizing copper nano-particles in 2011. The study aimed to obtain more stable copper nano-particles ink by using a Milestone microwave oven. The temperature applied was between $60^{\circ} \mathrm{C}$ and $170^{\circ} \mathrm{C}$ [51]. The results obtained in this study showed that the temperature has the greatest effect on the morphology and the growth of the copper nano-particles, which means that a microwave could be used to control the particle size [51]. 
Different sizes of copper nano-particles were used in a study conducted by Zuo in 2017. Copper nano-particles of $20 \mathrm{~nm}$ and $100 \mathrm{~nm}$ used in bonding paste are expected to improve the shear strength of the copper-copper bond. The modified joints with this optimal mixed paste show the highest bonding strength of over $15 \mathrm{MPa}$ at a lower temperature of $250^{\circ} \mathrm{C}$ and pressure of $4 \mathrm{MPa}$. Accordingly, paste containing particles of varying sizes could be a promising bonding material for the high-density packaging of electronic devices [52].

Cheng et al. used synthesized copper nano-particles reduced by 1-ascorbic acid and coated with PVP to obtain antioxidative copper nano-particles ink. The average size of particles achieved in this study was $140 \mathrm{~nm}$, and they showed significant stability after being stored for 3 months in the ambient condition. This can be attributed to the use of PVP and 1-ascorbic acid as a capping agent, which prevents the particles from growing and provides a shield against oxidizing when sintered [53]. Figure 1.8 presents XRD analyses of copper nano-particles stored for 0 months and 3 months.

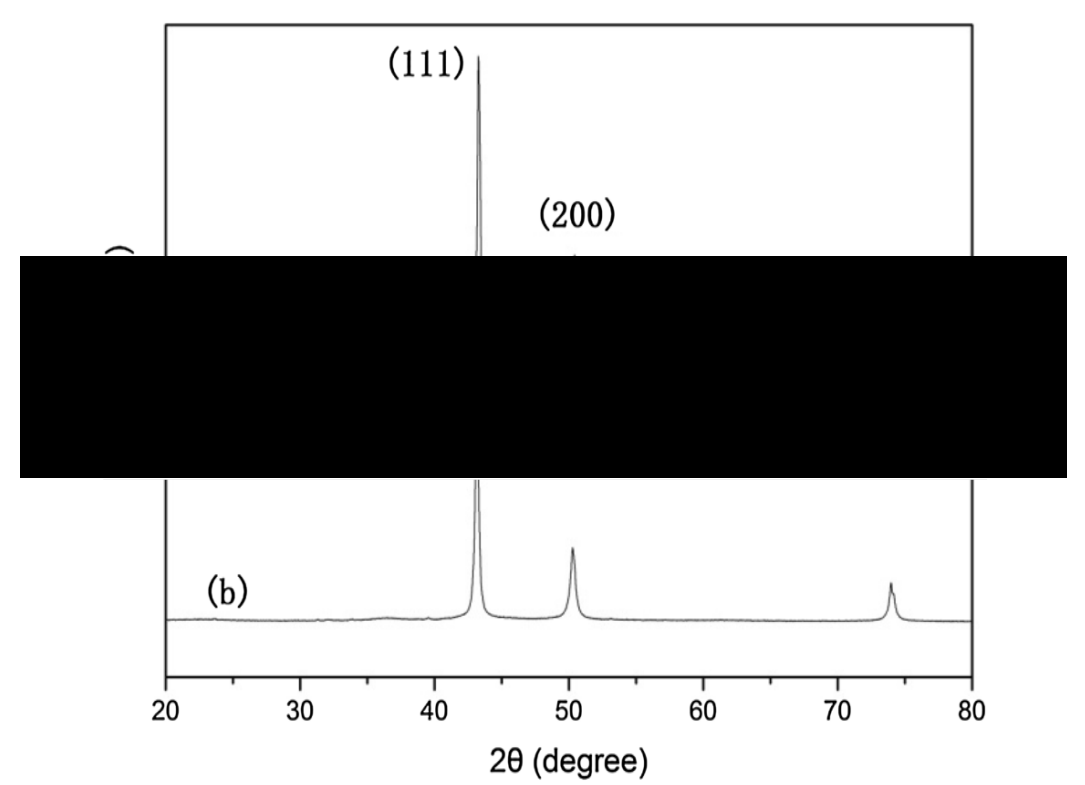

Figure 1.8 XRD analyses of copper nano-particles stored for (a) 0 months and (b) 3 months [53]

The synthesis of copper nano-particles ink has a significant influence on the deposition process, as it depends on the material viscosity. It also affects the sintering method, in which the size of particles plays an important role [21]. The next section will review these influences at sintering methods. 


\subsubsection{Thermal sintering of copper nano- and micro-particles}

Thermal energy is energy controlled by an object due to the movement of molecules or atoms within the object. It is one of the types of energy that can be used to sinter copper ink [54]. In this study, the aim of producing copper particles by applying heat is to enhance film conductivity. Since thermal energy is caused by the movement of particles, it is a type of kinetic energy, which is the energy caused by motion. Thermodynamic energy can be defined as an internal energy present in a system due to its temperature [55].

The sintering of printed copper nano-particles has recently received considerable attention due to the potential for saving cost [56] [57]. The rapid oxidation of copper nano-particles ink motivates the scientific researchers to utilize a reducing atmosphere during the thermal sintering [58] [53]. Recently, numerous reducing atmospheres have been employed to sinter printed copper, such as mixed gas $\left(\mathrm{H}_{2} / \mathrm{N}_{2}\right)$, and $\operatorname{argon}$. In the following section, reducing atmospheres will be reviewed individually.

\subsubsection{Thermal sintering under nitrogen reducing atmosphere}

To investigate the benefit of a nitrogen reducing atmosphere in sintering copper nanoparticles film, understanding the interaction between nitrogen and the surface of the copper film is a priority, as copper is widely used in systems containing nitrogen, such as the reduction and decomposition of nitrogen oxide [59]. Nitrogen is a very stable molecule [60], as the two nitrogen atoms in its molecules are united by a particularly strong triple bond. Furthermore, it prevents oxidation by applying nitrogen gas throughout the oven during the sintering process. As nitrogen is inert (non-reactive) and will displace the oxygen to prevent scale formation of copper oxide, a nitrogen atmosphere has been used in the nanostructure fabrication of silver nanowires [61]. Therefore, employing an inert gas such as nitrogen helps to reduce the oxygen [62] partial pressure, which can be used as an alternative condition to the high vacuum atmosphere, as it known to be more complicated and expensive [63].

In 2016, Yonezawa proved that a low sintering temperature of $100^{\circ} \mathrm{C}$ under a nitrogen reducing atmosphere of copper nano-particles deposited on alumina substrates using the doctor blade method led to low film resistivity of $9.0 \times 10^{-6} \Omega \mathrm{m}$. The sintering process was conducted using a tube furnace at a flow rate of pure nitrogen $1 \mathrm{dm}^{3}$ per 
min [64]. The sintering of copper nano-particles at a higher temperature of $200^{\circ} \mathrm{C}$ investigated by Lee et al. in 2015 also used the doctor blade technique under a nitrogen reducing atmosphere, but with controlling of the substrate temperature. The substrate used in this study was polyimide (PI), which was kept at $85^{\circ} \mathrm{C}$ during the printing process, allowing the ink to be sintered gradually during the printing and afterwards. The film resistivity achieved in this study was $45 \mu \Omega \mathrm{cm}$ after $20 \mathrm{~min}$ of sintering, then $3.6 \mu \Omega \mathrm{cm}$, or 2.2 times the resistivity of bulk copper, after $60 \mathrm{~min}$ of sintering [36].

In this study, the sintering process was carried out under a nitrogen reducing atmosphere at $200^{\circ} \mathrm{C}$ for $30 \mathrm{~min}$. The resistivity of the copper electrode was $16 \mu \Omega \mathrm{cm}$ [65]. Figure 1.9 shows the SEM images of the copper film on a glass substrate and pressure-less sintered at different temperatures under a nitrogen reducing atmosphere, which suggested that the best sintering temperature is $200^{\circ} \mathrm{C}$, as a higher temperature led to over-sintering.

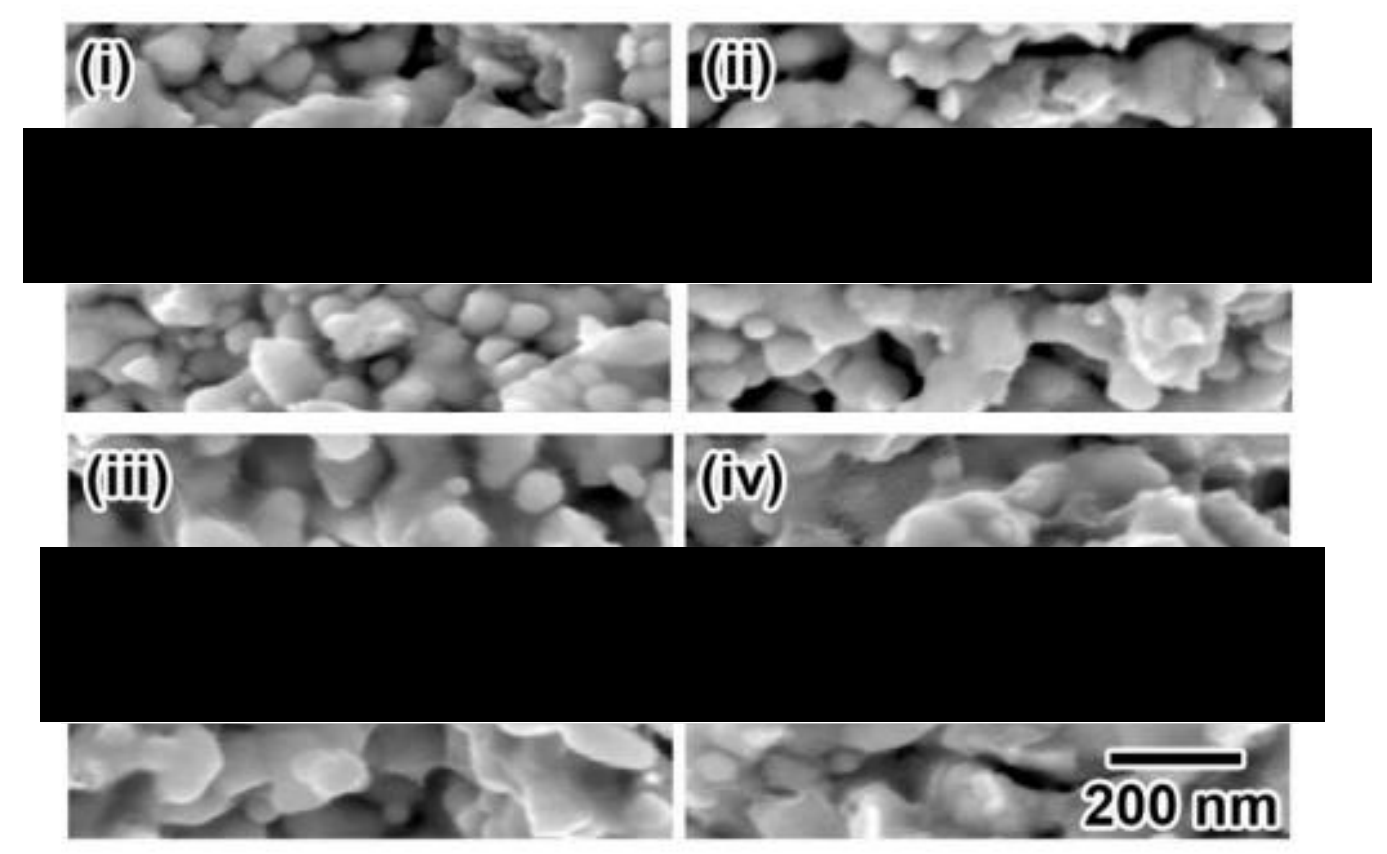

Figure 1.9 SEM images (i) $180^{\circ} \mathrm{C}$, (ii) $200^{\circ} \mathrm{C}$, (iii) $230^{\circ} \mathrm{C}$, and (iv) $260^{\circ} \mathrm{C}$ under a nitrogen reducing atmosphere for $30 \mathrm{~min}$ [65]

Printed copper on glass substrate was also used with the doctor blade technique by Arriffin et al. in 2010 . The samples were dried at $70^{\circ} \mathrm{C}$ for $15 \mathrm{~min}$ and then sintered 
under a nitrogen reducing atmosphere for $60 \mathrm{~min}$ at $300^{\circ} \mathrm{C}$. The resistivity improved compared to the sintering under an ambient atmosphere [66].

Most of the studies have shown that it is possible to employ nitrogen to sinter copper nano-particles, which were deposited by screen, inkjet and doctor blade printed features, but that this comes at the expense of conductivity, with the best conductivity being 10-15 times higher than bulk copper resistivity $\left(9.0 \times 10^{-6} \Omega \mathrm{m}\right)$ [64]. Consequently, other reducing atmospheres, such as an $\mathrm{H}_{2} / \mathrm{N}_{2}$ mixed gas or formic acid environment, have been employed by many researchers in sintering copper ink [53].

\subsubsection{Thermal sintering under formic acid atmosphere}

Reduction processes using formic acid are widely used in semiconductor and PCB manufacturing [67]. Employing a formic acid atmosphere motivated many researchers, benefiting from the reducing agent on sintering copper nano-particles film [68]. However, understanding the mechanism of formic acid decomposition and the behaviour of the copper film under this atmosphere is a priority, as this could affect the substrate used. The main aim is for the copper oxide to be removed by the chemical reaction with formic acid. The reaction process in the presence of formic acid as the reducing agent [69] to prevent copper oxidation is as follows:

$$
\begin{array}{rlr}
\mathrm{HCOOH} & \rightarrow \mathrm{CO}_{2}+\mathrm{H}_{2} & \ldots \text { Eq. } 1.4 \\
\mathrm{HCOOH} & \rightarrow \mathrm{CO}+\mathrm{H}_{2} \mathrm{O} & \ldots \text { Eq. } 1.5 \\
\mathrm{Cu}^{2+}+2 \mathrm{HCOOH} & \rightarrow \mathrm{Cu}+\mathrm{H}_{2}+2 \mathrm{HCO}_{2}- & \ldots E q .1 .6 \\
\mathrm{CuO}+2 \mathrm{HCOOH} & \rightarrow \mathrm{Cu}(\mathrm{COOH})_{2}+\mathrm{H}_{2} \mathrm{O} & \ldots \text { Eq. } 1.7
\end{array}
$$

Therefore, formic acid vapour can provide a significant reducing atmosphere [62]. In other studies, a formic acid atmosphere was applied directly into a copper ink or just before the printing took place [70].

Decomposition of formic acid at high temperature and its reactions with different adsorbed species provide a sufficient reducing environment inside the oven. The aim is to enhance the conductivity of copper film by preventing the oxidation reaction. On the other hand, a disadvantage of using formic acid is that it might lead to significant 
decolouration of an FTO substrate, as the high-temperature acidic vapour (which has not thermally decomposed to $\mathrm{CO}$ and $\mathrm{H}_{2} \mathrm{O}$ ) might react with the FTO coating and cause yellowing of the substrate [21]. Thermal sintering allows the copper nanoparticles or micro-particles to fuse and creates a good path for the current to travel through once the oxygen is disabled to create a barrier by forming copper oxide, which can be generated under a formic acid atmosphere [49].

A low sintering temperature study was conducted by Hermerschmidt et al. They used a formic acid atmosphere to sinter nano-copper, which was inkjet printed on ITOcoated glass. The conductivity achieved with sintering at $130^{\circ} \mathrm{C}$ up to $16 \%$ of the conductivity of bulk copper and more than $25 \%$ was observed with sintering at $150^{\circ} \mathrm{C}$. Adhesion and bending tests confirmed the stability of the thin film and up to $2 \%$ tensile strain. The study aimed to achieve a low-cost printable material sintered at low temperature compatible with plastic substrates such as polyethene terephthalate [71].

Another study investigated the effect of formic acid on the sintering of copper nanoparticles ink, but as a pre-treatment on the ink preparation. The spherical copper nanoparticles with an average size of $30 \mathrm{~nm}$ were immersed in a mixed solution of formic acid and absolute ethanol [68]. The study reported that the formic acid pre-treatment enabled the sintering process under mixed $95 \% \mathrm{~N}_{2} 5 \% \mathrm{H}_{2}$ to obtain film resistivity of $3.6 \mu \Omega \mathrm{cm}$ at a low temperature $\left(160^{\circ} \mathrm{C}\right)$. In addition, the resistivity increased to 6.12 $\mu \Omega \mathrm{cm}$ when the sintering temperature was increased to $260^{\circ} \mathrm{C}$. This also resulted in excellent adhesion performance on plastic or glass substrates. The main finding was that the formic acid pre-treatment method is more effective than the technique using formic acid vapour as a reducing atmosphere [68].

Lee conducted a study using inkjet printing [72]. However, comparing Lee's study with Liu's study is not possible, as Lee sintered the copper nano-particles ink at $200^{\circ} \mathrm{C}$ for $1 \mathrm{~h}$ in a tube furnace under a reducing atmosphere of nitrogen gas bubbled through formic acid. In contrast, Liu employed dual reducing parameters (formic acid as pretreatment and a gas mixture of $95 \% \mathrm{~N}_{2} 5 \% \mathrm{H}_{2}$ ) as a sintering atmosphere for $10 \mathrm{~min}$ [68]. Figure 1.10 shows the resistivity obtained under a formic acid atmosphere of the copper pattern on a PI substrate as a function of time sintered at $200^{\circ} \mathrm{C}$ under a reducing atmosphere [72]. 


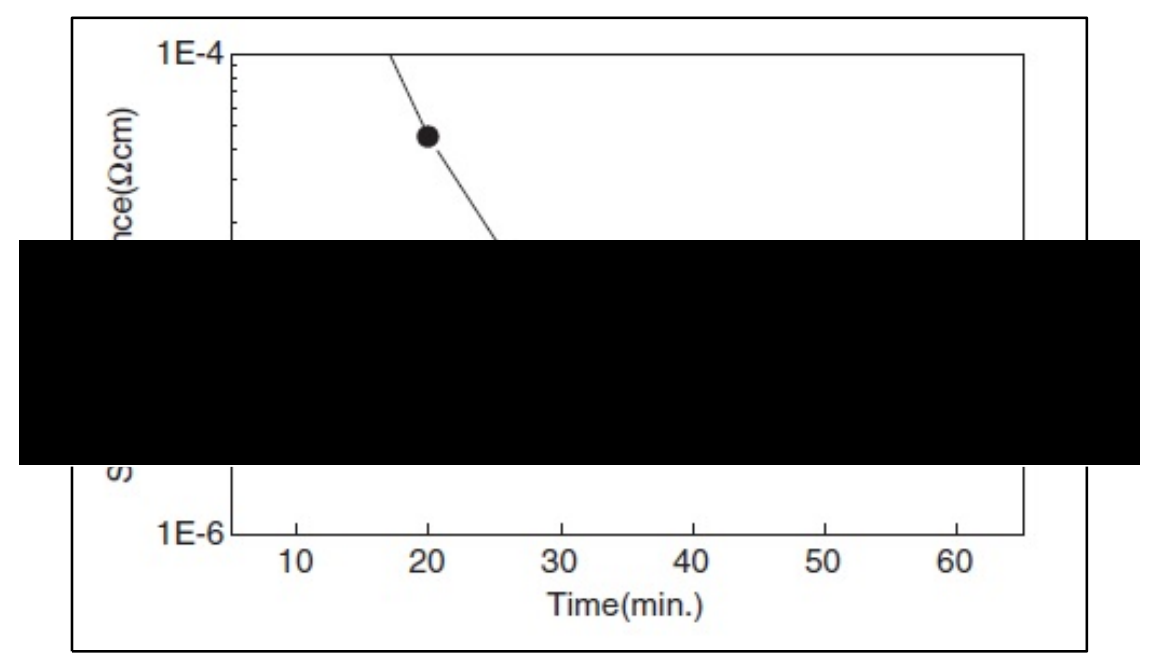

Figure 1.10 Resistivity of copper pattern on a PI substrate as a function of time sintered at $200^{\circ} \mathrm{C}$ under a reducing atmosphere [72]

Kim et al. reported a more straightforward method of using formic acid during the sintering process in 2012. The printed sample and a petri dish containing formic acid were placed on a hot plate and then covered with a larger petri dish. A glass substrate was used, and the deposition was conducted by the bar coating method. The annealing process was carried out at $200^{\circ} \mathrm{C}$ for $2 \mathrm{~min}$ on a hot plate in a glove box followed by reduction with formic acid gas at $250^{\circ} \mathrm{C}$ for $2-10 \mathrm{~min}$. [73] The lowest resistivity achieved was $5.2 \mu \Omega \cdot \mathrm{cm}$ at $200^{\circ} \mathrm{C}$ for 2 min followed by reduction with formic acid gas at $250^{\circ} \mathrm{C}$ for $2 \mathrm{~min}[73]$.

\subsubsection{Thermal sintering under mixed gas atmosphere}

This section reviews the sintering process under a reducing atmosphere by employing a two-component mixture such as $95 \% \mathrm{~N}_{2} / 5 \% \mathrm{H}_{2}$ or an $96 \% \mathrm{Ar} / 4 \% \mathrm{H}_{2}$ gas mixture [53]. The $\mathrm{H}_{2} / \mathrm{N}_{2}$ gas mixture has the benefit of low surface oxidation, as hydrogen acts as a scavenging agent, preventing the formation of a thick chrome oxide layer when it is used in the manufacturing of austenitic stainless steels [74]. The low surface oxidation is a clear and consistent benefit of using the more controlled reducing atmosphere produced by the $5 \% \mathrm{H}_{2} / 95 \% \mathrm{~N}_{2}$ mixture [75].

A mixture of $96 \% \mathrm{Ar} / 4 \% \mathrm{H}_{2}$ was used in a sintering atmosphere study conducted by Cheng et al. [53]. The copper ink utilized in this study has been described in section 
1.3.5. The copper film on the flexible substrate was sintered in a muffle furnace with varying temperatures $\left(200^{\circ} \mathrm{C}-400^{\circ} \mathrm{C}\right)$ and times $(10 \mathrm{~min}-180 \mathrm{~min})$. The lowest resistivity achieved was $5.7 \mu \Omega \mathrm{cm}$ within $30 \mathrm{~min}$ at a temperature of $400^{\circ} \mathrm{C}$ under the mixed gas sintering atmosphere. The study suggested that resistivity of three times greater than the electrical resistivity of bulk copper $(1.75 \mu \Omega \mathrm{cm})$ can be obtained by employing the mixture gas of $96 \% \mathrm{Ar} / 4 \% \mathrm{H}_{2}$ sintering atmosphere [53]. Figure 1.11 shows the variations in electrical resistivity of copper films under a specific temperature as sintering time increases.

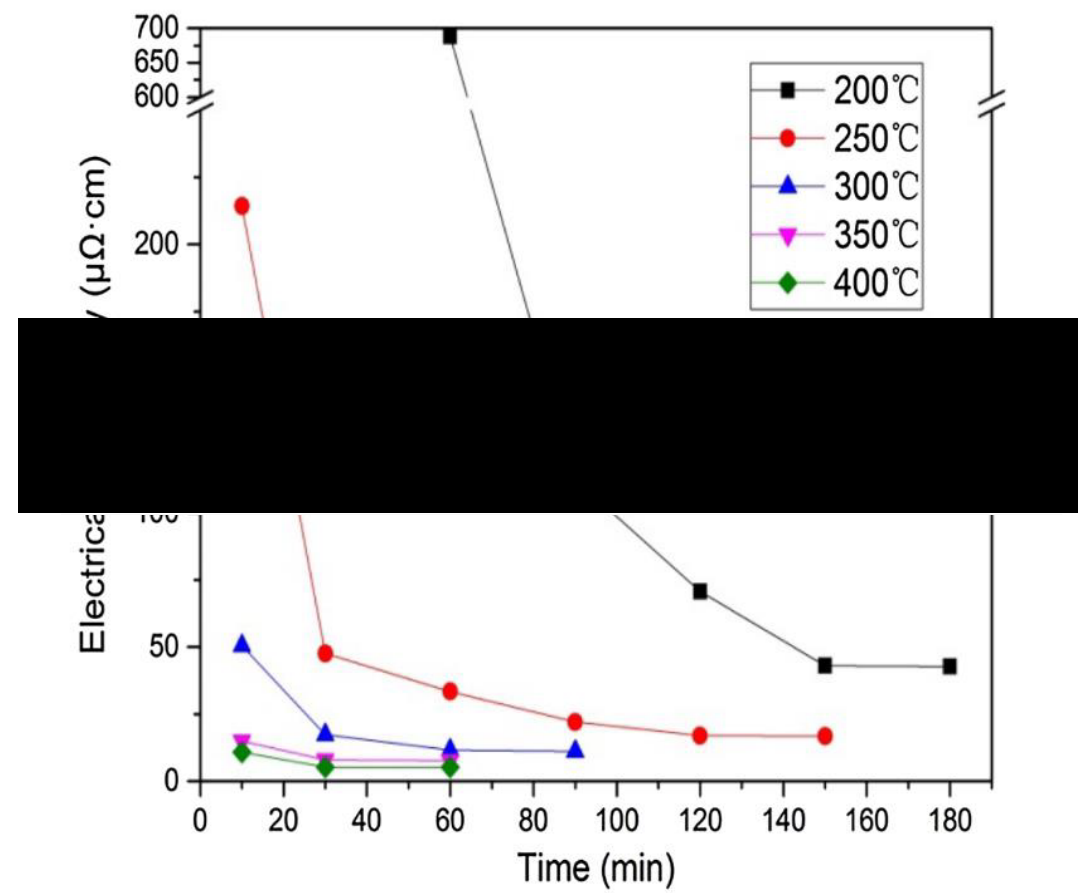

Figure 1.11 The variations in electrical resistivity of copper films under a certain temperature as sintering time increases under $96 \% \mathrm{Ar} / 4 \% \mathrm{H}_{2}$ [53]

The effect of using a sintering temperature under a condition of $5 \% \mathrm{H}_{2} / 95 \% \mathrm{~N}_{2}$ was reported by Liu in 2016 [76]. Using direct bonding copper (DBC) substrate and SiC components, the copper films were dried and sintered under this atmosphere, with a short sintering time at $200^{\circ} \mathrm{C}, 260^{\circ} \mathrm{C}$ and $320^{\circ} \mathrm{C}$ for $5 \mathrm{~min}$. The film resistivity increased from $40.5 \mu \Omega \cdot \mathrm{cm}$ to $5.65 \mu \Omega \cdot \mathrm{cm}$ when the sintering temperature was increased from $200^{\circ} \mathrm{C}$ to $260^{\circ} \mathrm{C}$ [76]. 
Overall, the high cost of thermal sintering encourages the exploration of alternative sintering methods [77] to determine whether copper nano-particles could be continuously processed. Several studies have been conducted using high power broad area pulsed visible light photonic emission [10], which offers almost instantaneous sintering without the register required by laser sintering [78].

\subsubsection{Photonic sintering of copper nano- and micro-particles}

Photonic sintering is one of the fastest processes that can be used to sinter copper nano-ink [79]. Using white intense pulsed light from a xenon lamp has another advantage in addition to the fast sintering, as it avoids causing any damage to the substrate during the sintering process [10].

The generation of the light energy is correlated with the size of the capacitors, the voltage applied to the capacitor, and the number of pulses. Figure 1.12 shows the lightgenerating diagram.

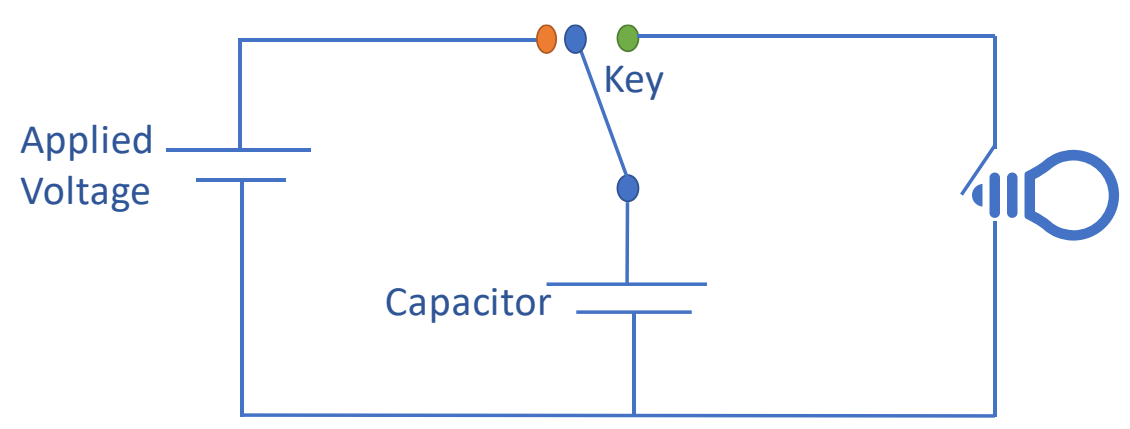

Figure 1.12 Light-generating diagram

The PulseForge photonic curing system, which is one of the most common techniques utilized to sinter thin copper film, is based on light energy [80] [21]. The design and configurability of PulseForge allow the sintering process to be valid independently of the deposition method used for the material being processed [81]. PulseForge also enables the use of less material to reach the target conductivity in conjunction with the faster processing time; consequently, a significant product cost saving can be achieved [10]. The basis of PulseForge's ability is using light energy to sinter metallic ink. It also has the capability to deliver significant energy to the surface of the application in a short time. 
A multi-pulsed light sintering process on inkjet-printed copper nano-ink (30 nm) with fixable plastic substrate was used in a study conducted by Han et al. With maximum energy of $100 \mathrm{~J} / \mathrm{cm}^{2}$ applied, the temperature of the surface material reached $1050^{\circ} \mathrm{C}$ in $20 \mathrm{~ms}$. The sintering was conducted at different light intensities with active energy of $17-32 \mathrm{~J} / \mathrm{cm}^{2}$. Figure 1.13 shows how the content responds according to the applied energy [82].

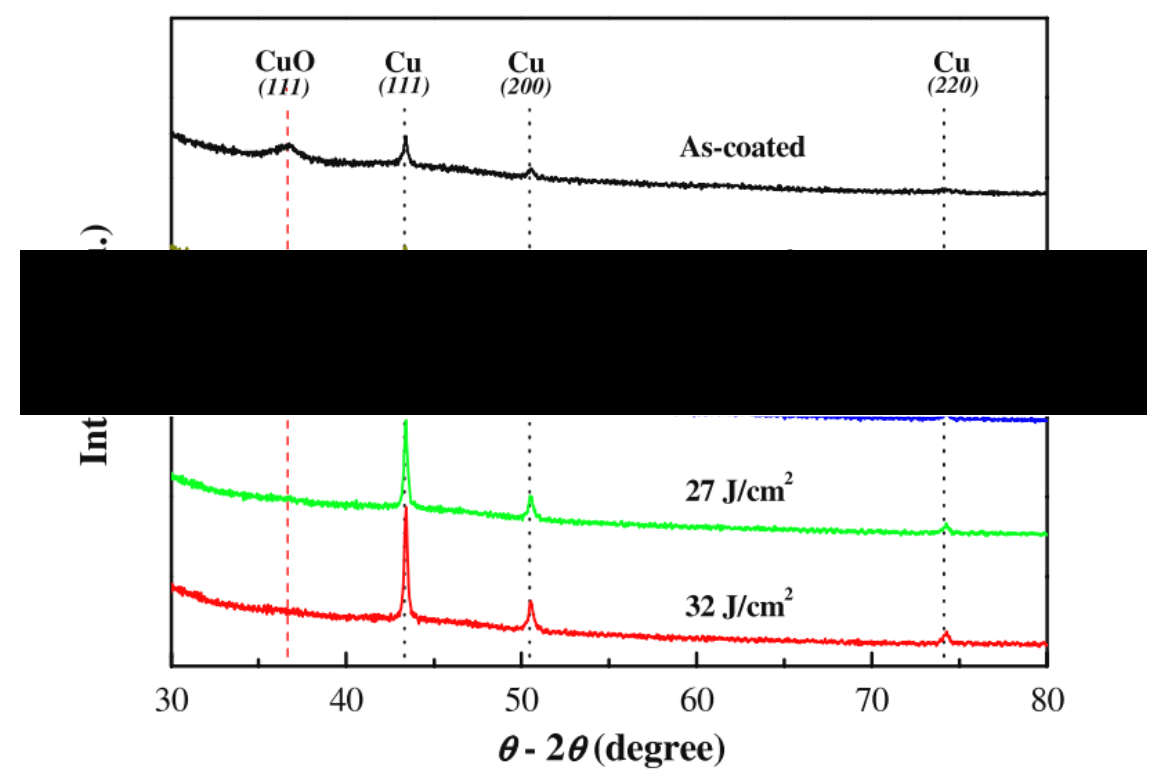

Figure 1.13 XRD of copper nano-ink sintered at different light intensities [82]

Slight damage occurred on the PI substrate at the energy level higher than $50 \mathrm{~J} / \mathrm{cm}^{2}$. However, the best electrical structure was obtained with sample (e) in Table 1.1; nevertheless, eight pulses led to the total energy of $32 \mathrm{~J} / \mathrm{cm}^{2}$. The lowest resistivity obtained was about 100 times higher than with bulk copper, which confirms that photonic sintering relies not only on the applied energy, but also on the mechanism and the crystallization of the materials. It can be dependent on the explosive surface melting and condensation process. Therefore, the resultant nano-structures have many defects that act as a barrier to electron movement, which affects the current flow [83]. 
Table 1.1 The summary of sample identification (ID) and pulse conditions to the total energy achieved [82]

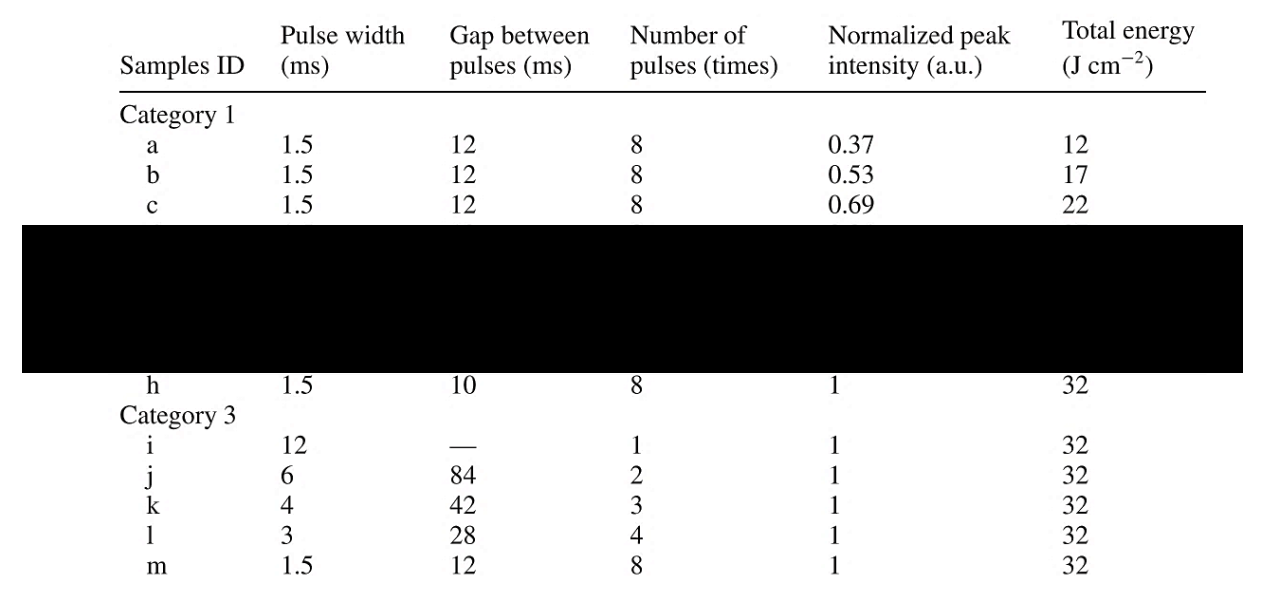

The principal finding in Han's research is that high energy could lead to over-sintering or poor conductivity [82]. However, another study with low-energy photonic sintering conducted by Chung in 2015 used hybrid copper ink with nano-particles of $10 \mathrm{~nm}-$ $70 \mathrm{~nm}$, and the ink was deposited on a PI substrate using the doctor blade printing method. Samples were then dried on a hot plate at $80^{\circ} \mathrm{C}$ for $2 \mathrm{~h}$. The sintering process using flashlight sintering (PerkinElmer co) was conducted at room temperature with

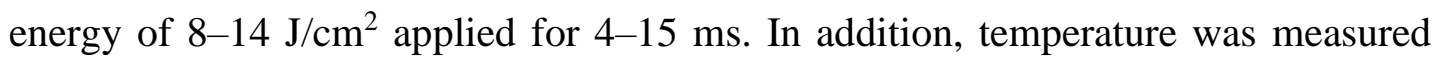
during the sintering process using a non-inverting amplifier circuit. The best resistivity achieved was $27.3 \mu \Omega \mathrm{cm}$. Figure 1.14 shows resistivity at different pulse times [10].

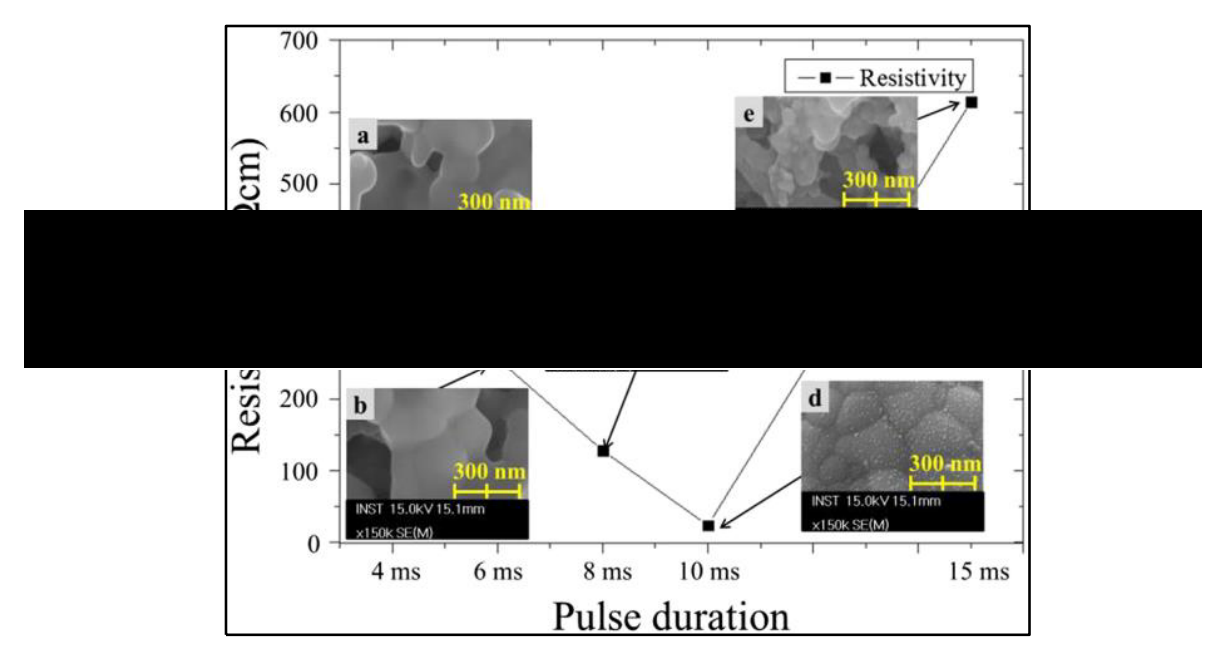

Figure 1.14 Copper resistivity as function of pulse duration (4-15 ms) [10] 
A study investigating the temperature change during the flashlight sintering process was carried out using a PI substrate and the doctor blade printing method [7]. Park stated that in this study, the copper nano-particles films were agglomerated and fully sintered at sintering energy of $12 \mathrm{~J} / \mathrm{cm}^{2}$ with no damage caused to PI substrates [7]. Figure 1.15 shows the agglomeration level in responding to the applied energy.

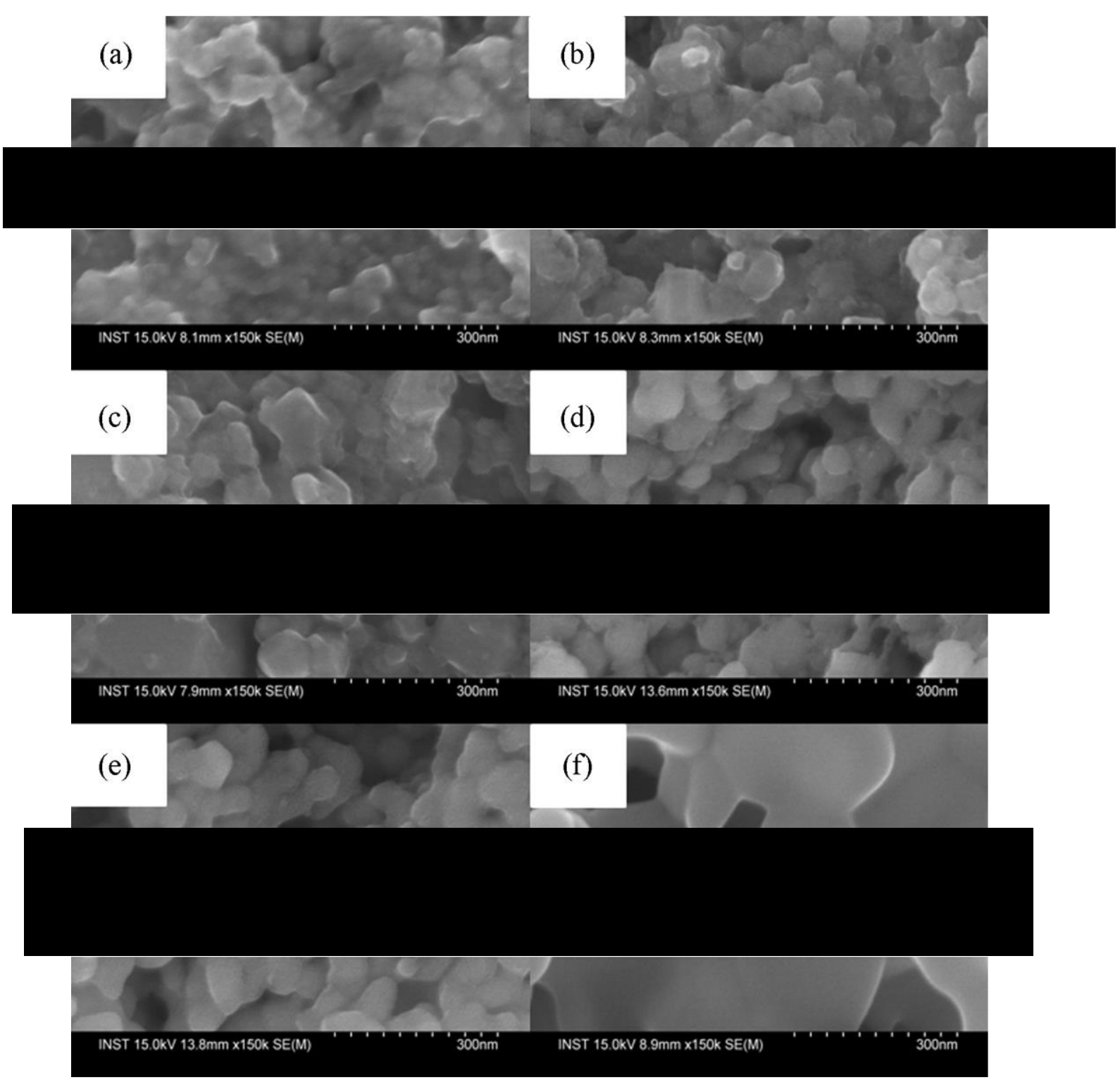

Figure 1.15 SEM images of the flashlight irradiated copper nanofilms as changing irradiation energy; (a) $2 \mathrm{~J} / \mathrm{cm}^{2}$, (b) $4 \mathrm{~J} / \mathrm{cm}^{2}$, (c) $6 \mathrm{~J} / \mathrm{cm}^{2}$, (d) $8 \mathrm{~J} / \mathrm{cm}^{2}$, (e) $10 \mathrm{~J} / \mathrm{cm}^{2}$ and (f) $12 \mathrm{~J} / \mathrm{cm}^{2}$ [7] 
The skin depth of the material in a deposited thick or thin film is considered a vital parameter of the physical kinetics when photonic energy is applied to sinter the film. A highly reflective surface could affect the energy required to sinter thick films and the stability of the film [84]. This condition must be considered when thick metallic films are deposited on a glass substrate. It is necessary to study the optical conductivity and the complex refractive or complex dielectric function for the copper nano-particles ink. These physical parameters are temperature- and wavelength-dependent [84]. Figure 1.16 illustrates the skin effect region, the emissivity of copper and silver, which shows a broad peak in the mid-infrared at $25^{\circ} \mathrm{C}$.

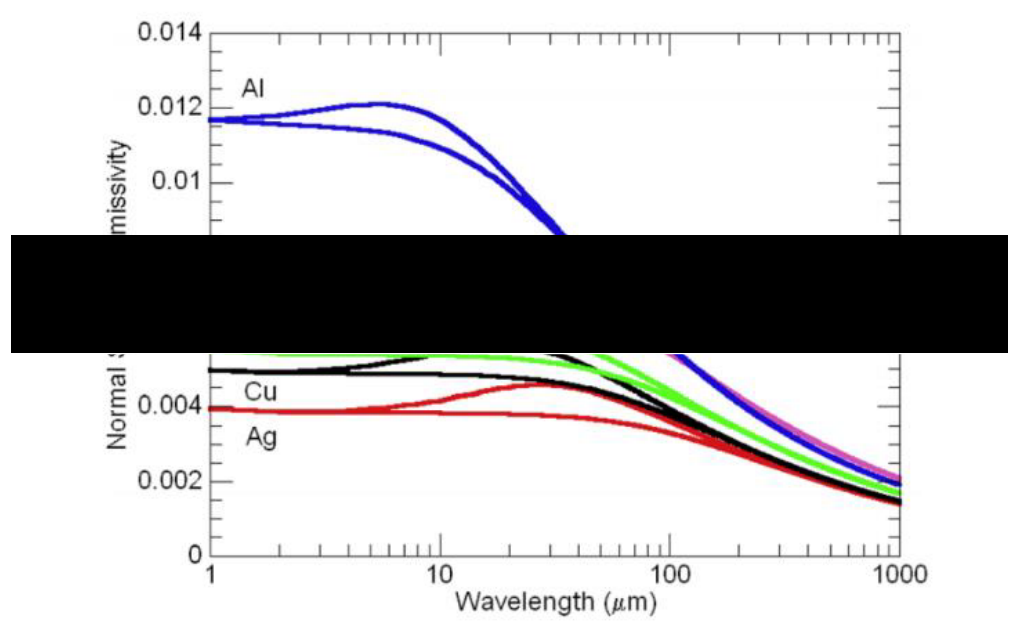

Figure 1.16 The skin effect region, the emissivity of copper and silver [84]

Ennaceri et al. carried out a study to improve the first surface of silvered thick glass mirrors and used $2 \mathrm{~mm}$ glass covered with a metal back layer of copper (50 nm) as back protection of the mirror. A reflective layer of silver $(100 \mathrm{~nm})$ was deposited on top of the copper layer using the sputtering PVD (Physical vapour deposition) technique. The main finding shows that the prepared mirrors with different combinations have similar specular reflectance, with a high value $(98.6 \%)$ [85].

The spectral dependence of the light extinction, scattering, and absorption efficiency factors was studied by Kalenskii et al. in copper nano-particles synthesis [86]. They demonstrated that the plasmon extinction band on the spectral dependence is shifted with increasing nano-particle radius, which is followed by the band broadening if the maximum of the extinction efficiency factor lies at wavelengths greater than $600 \mathrm{~nm}$ [86]. 
The optical properties of copper oxide thin film were investigated by Kayani et al., who studied the optical conductivity of undoped and V-doped copper oxide thin films as a function of skin depth. Table 1.2 shows that the optical conductivity was increased with the combination of $\mathrm{V}$ doping and lower skin depth. This is attributed to the enhancement in crystallization and voids produced in thin films [87].

Table 1.2 Optical properties of undoped and V-doped copper oxide thin films [87]

\begin{tabular}{|c|c|c|c|c|c|c|c|}
\hline $\begin{array}{l}\text { V Conc. } \\
\text { (\%) }\end{array}$ & $\begin{array}{l}\text { Band gap energy } \\
\text { (eV) }\end{array}$ & $\begin{array}{l}\text { Film thickness } \\
(\mu \mathrm{m})\end{array}$ & $\begin{array}{l}\text { Extinction } \\
\text { coefficient K }\end{array}$ & $\begin{array}{l}\text { Optical conductivity } \sigma \\
(\mathrm{S} / \mathrm{cm})\end{array}$ & $\begin{array}{l}\text { Urbach energy } E_{U} \\
(\mathrm{meV})\end{array}$ & $\begin{array}{l}\text { Skin depth (cm) } \\
\times 10^{-4}\end{array}$ & $\begin{array}{l}\text { Refractive } \\
\text { index } n\end{array}$ \\
\hline & 106 & 180 & ברתחم & م.175 & 1100 & 31 & 150 \\
\hline 4 & 1.68 & 3.82 & 0.044 & 0.807 & 324 & 1.5 & 1.72 \\
\hline 5 & 1.64 & 3.96 & 0.042 & 0.691 & 344 & 1.6 & 2.14 \\
\hline
\end{tabular}

The flashlight process can also be used with multi-step processing [88], and a twostep flashlight sintering process was employed to reduce the warping of polymer substrates that can be caused by thermal sintering. To identify the best sintering conditions of the copper nano-ink, the flashlight irradiation conditions were applied at different power, pulsed number, and a different time. To monitor the flashlight sintering process, in-situ resistance and temperature monitoring of copper nano-ink were conducted during the flashlight sintering process. The finite-element program ABAQUS was used to predict the temperature changes of copper nano-ink and the temperature of the polymer substrate. The resulting two-step flashlight sintered copper nano-ink films exhibited low resistivity $(3.81 \mu \Omega \mathrm{cm})$ and good adhesion strength without warping of the polymer substrate [89].

The main challenge in using photonic sintering is that the light energy is applied to the whole sample surface and that energy is dependent on the size of the printed feature, whereas laser sintering comes with a selective condition for each printed object [21].

\subsubsection{Laser sintering of copper nano- and micro-particles}

The challenge with using the flashlight in copper sintering encourages researchers to try a variety of techniques as an alternative [90] [91]. Laser sintering is one of the fast sintering methods [92] [93] [94] [95] and it has the ability to select the surface area. 
Printed copper nano-particles film sintered by employing a laser [96] [97] in both an ambient and a nitrogen atmosphere have been investigated in several studies. The following paragraphs will discuss laser sintering.

Halonen et al. studied the impact of laser sintering on copper nano-ink in an ambient condition. A diode laser was used with continuous-wave $808 \mathrm{~nm}$ to sinter copper film deposited on a PI substrate by an inkjet printing process. The sintering was conducted with power up to $35 \mathrm{~W}$ and the beam size was $2.5 \mathrm{~nm}$, which led to a laser spot of 1.1 $\mathrm{mm} \times 0.4 \mathrm{~mm}$. The samples were examined traditionally by using a multi-meter to measure the resistance. The resistivity obtained in this study decreased frequently as the optical power increased [91]. The copper resistivity at the power of $2.62 \mathrm{~W}$ was $53.7 \Omega$, and at $7.95 \mathrm{~W}$, it was 11-12 $\Omega$. Laser sintering forms larger grain structures with a good agglomeration level, which enhance the conductivity. The study indicated that laser sintering is a good technique to use in copper sintering and has a good repeatability effect on the conductivity of the copper film. However, the multiple sintering cycles at constant power and velocity showed no effect on the conductivity of the film [91]. Figure 1.17 presents the resistance of laser-sintered samples as a function of the optical power of the laser diode. The scanning velocities used were 50 $\mathrm{mm} / \mathrm{s}, 100 \mathrm{~mm} / \mathrm{s}, 200 \mathrm{~mm} / \mathrm{s}$, and $400 \mathrm{~mm} / \mathrm{s}$.

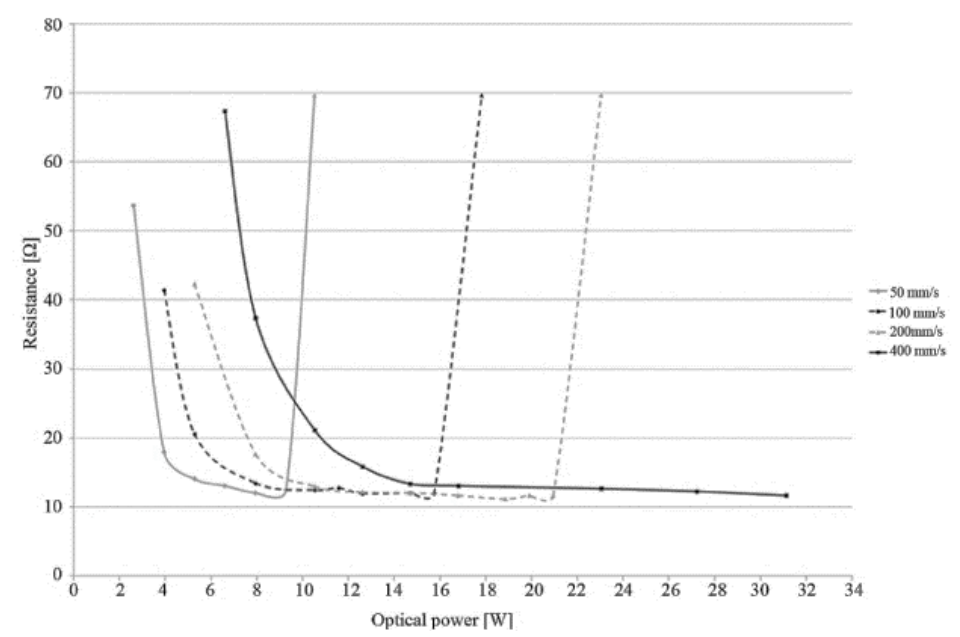

Figure 1.17 The resistance of laser-sintered samples as a function of the optical power of the laser diode $(50 \mathrm{~mm} / \mathrm{s}, 10, \mathrm{~mm} / \mathrm{s}, 200 \mathrm{~mm} / \mathrm{s}, 400 \mathrm{~mm} / \mathrm{s})$ 
In 2011, Joo et al. investigated laser sintering under a nitrogen atmosphere. Copper paste was screen printed on a PI substrate. An ultraviolet laser at $355 \mathrm{~nm}$ with different power applied was employed in this study to sinter the copper complex consisting of $\mathrm{Cu}$ and $\mathrm{Cu}(\mathrm{COOH})^{2}$, and nitrogen gas was blowing during the laser sintering to provide a reducing atmosphere during the sintering process. The best resistivity achieved in this study was $1.86 \times 10^{-5} \Omega$ at $275^{\circ} \mathrm{C}$. The XRD examination in figure 1.18 shows a significant difference between the film that was thermally sintered in the ambient air and the film sintered by laser under the nitrogen atmosphere, which shows no oxide peak [98].

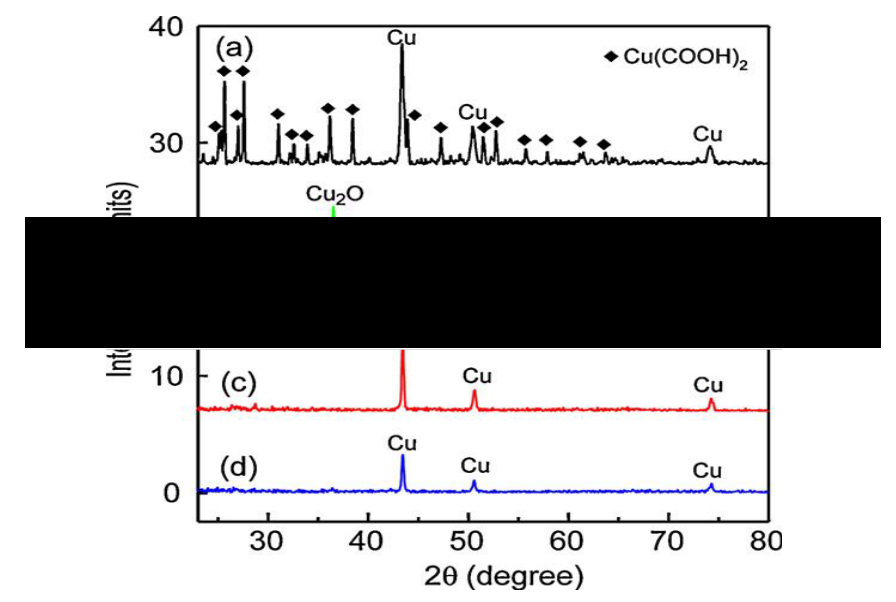

Figure 1.18 XRD. (a) As-printed, (b) thermally sintered in ambient air, (c) thermally sintered in $\mathrm{N}_{2}$ atmosphere, (d) laser-sintered in $\mathrm{N}_{2}$ atmosphere [98]

In Figure 1.19, the SEM image shows that no measurable change in the colour and surface morphology was observed at low power. However, with sintering at a power higher than $0.8 \mathrm{~W}$, the colour of the film changed slightly, and individual complex polyhedrons became porous, This could be attributed to the decomposition of formate and the removal of terpineol added to the ink [98]. 


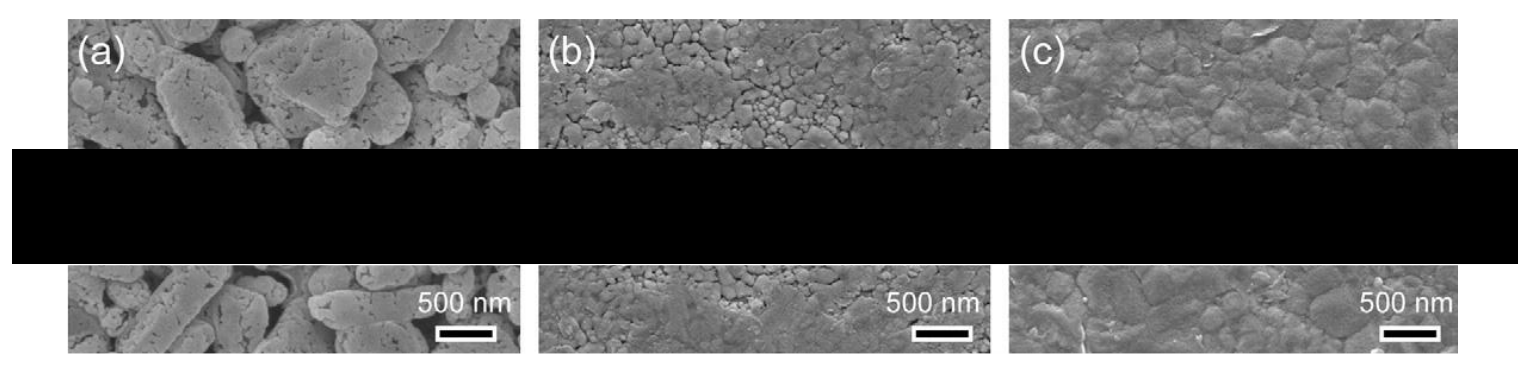

Figure 1.19 SEM image of films sintered at different laser powers of (a) $0.8 \mathrm{~W}$, (b)

$1.0 \mathrm{~W}$, and (c) $1.4 \mathrm{~W}[98]$

Both the thermal sintering process and the laser sintering process were carried out under a nitrogen atmosphere by Joo. The lowest resistivity achieved with the laser sintering was $1.41 \times 10^{-5} \Omega \mathrm{cm}$; this value was higher than the resistivity $\left(1.30 \times 10^{-5}\right.$ $\Omega \mathrm{cm}$ ) of the film thermally sintered at $275^{\circ} \mathrm{C}$ [16]. Figure 1.20 shows the comparison of the laser and thermal sintering sheet resistance achieved in this study.

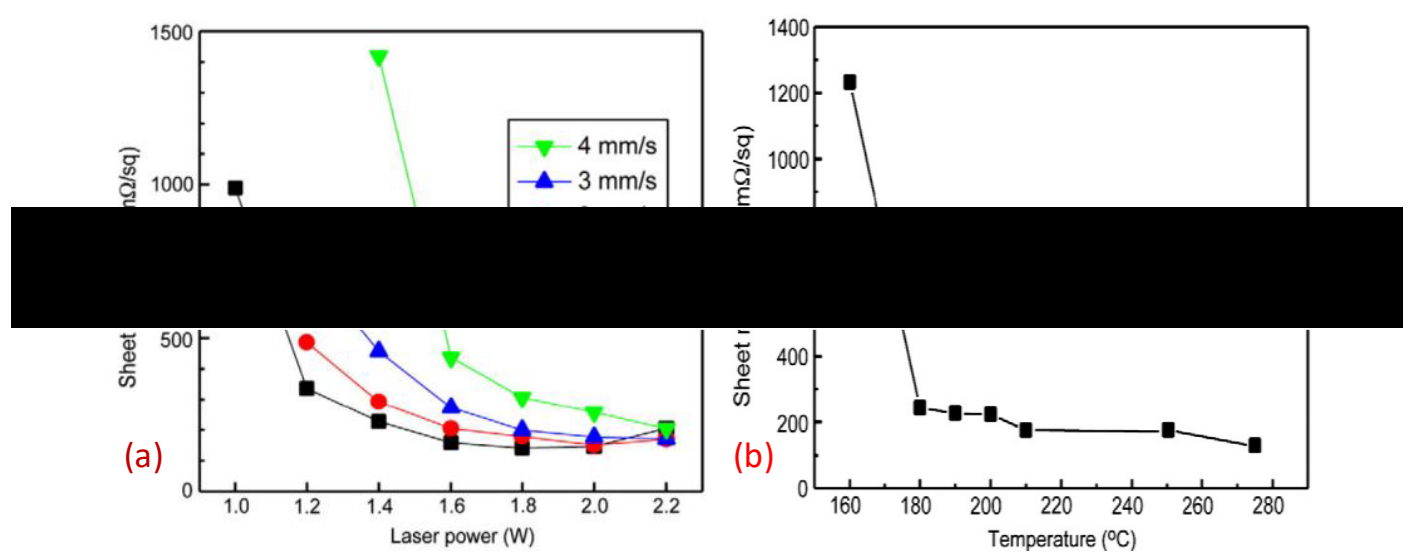

Figure 1.20 (a) Sheet resistances plotted as a function of the scan rate and laser power

(b) Sheet resistance as a function of the annealing temperature under $\mathrm{N}_{2}$ atmosphere [16]

Proving that better conductivity is achieved under an ambient condition requires further investigation [16].

In 2014, Zenou et al. used a laser for drying printed copper nano-particles under an ambient condition. In this study, a new conductivity measurement method was employed based on reflectance, and the ink was dried after printing by applying low laser power over the ink and keeping the laser spot size and scanning velocity fixed. The reason for using a low-power laser is to avoid boiling the solvent, which could 
lead to a degraded sintered pattern [99]. Figure 1.21 shows the SEM image of laser drying conditions on copper ink films for three different drying levels.

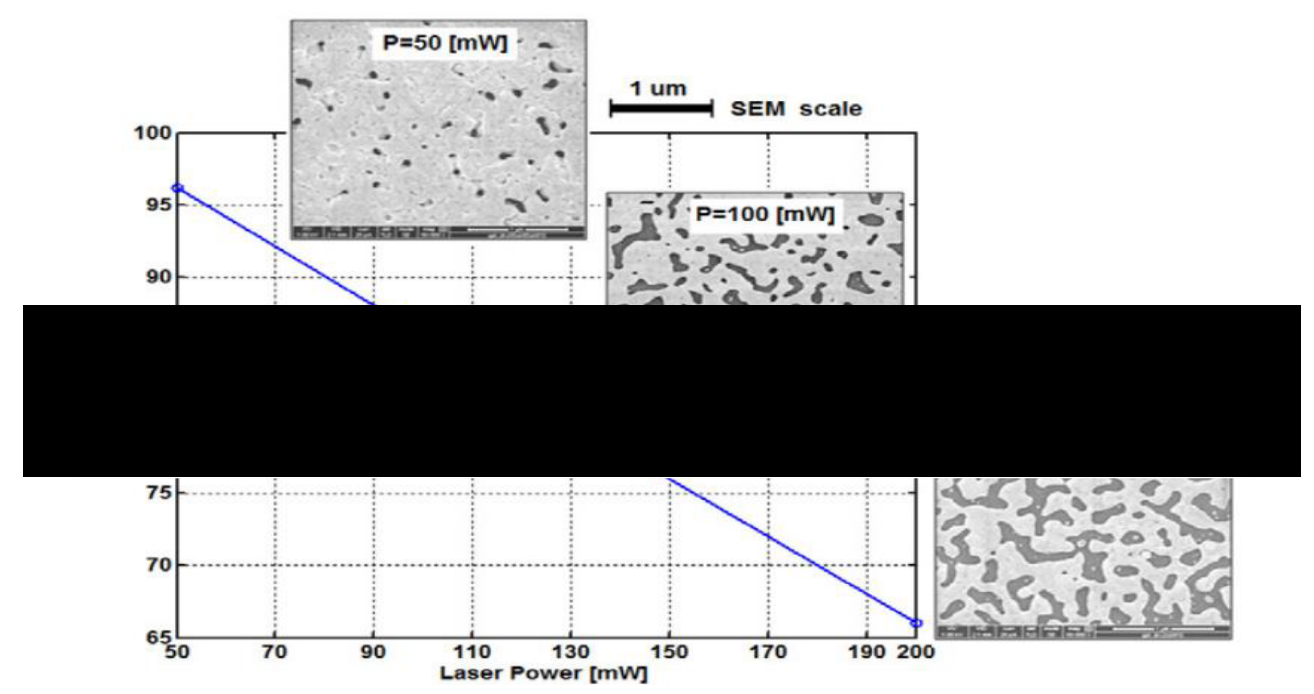

Figure 1.21 The effect of ink drying conditions on sintered $\mathrm{Cu}$ ink film for three different drying levels [99]

This study highlighted the potential of sintering copper nano-ink under an ambient condition, as the film resistivity achieved two-three times that of bulk copper by using a laser of $30-50 \mathrm{~J} / \mathrm{cm}^{2}$ for $5 \mathrm{~ms}$ [99]. Figure 1.22 shows the copper line resistivity dependence on laser power for scan velocity and illustrates the film ablation caused when high laser power is applied. 

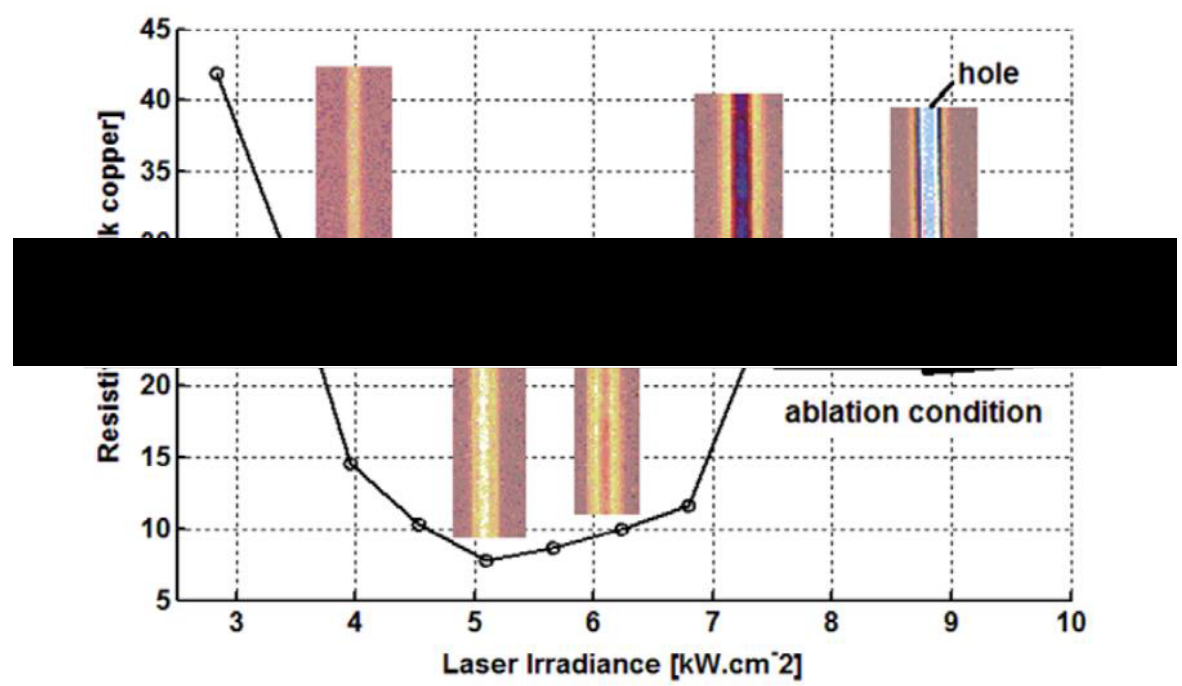

Figure 1.22 Copper line resistivity versus laser irradiance (constant laser scan velocity, $\mathrm{v}=3 \mathrm{mms}^{-1}$ ). The insets are bright field microscopy images of the scanned copper line [99]

Another method was proposed by Cheng et al. in 2016 using a femtosecond laser to sinter copper nano-particles. Femtosecond laser sintering is one of the most suitable technologies for reducing the surface oxidation by removing the solvent from the ink in a short time. The copper was deposited by spin coating onto a glass substrate, and then the samples were dried using a hot plate for only $30 \mathrm{~s}$ at $110^{\circ} \mathrm{C}$. The laser was applied under an ambient condition with $800 \mathrm{~nm}$ wavelengths and a repetition rate of $80 \mathrm{MHz}$. The reflectivity decreased when the laser pulse was applied [100]. Figure 1.23 shows the calculated surface reflectivity and absorption coefficient for a singleshot laser pulse. 


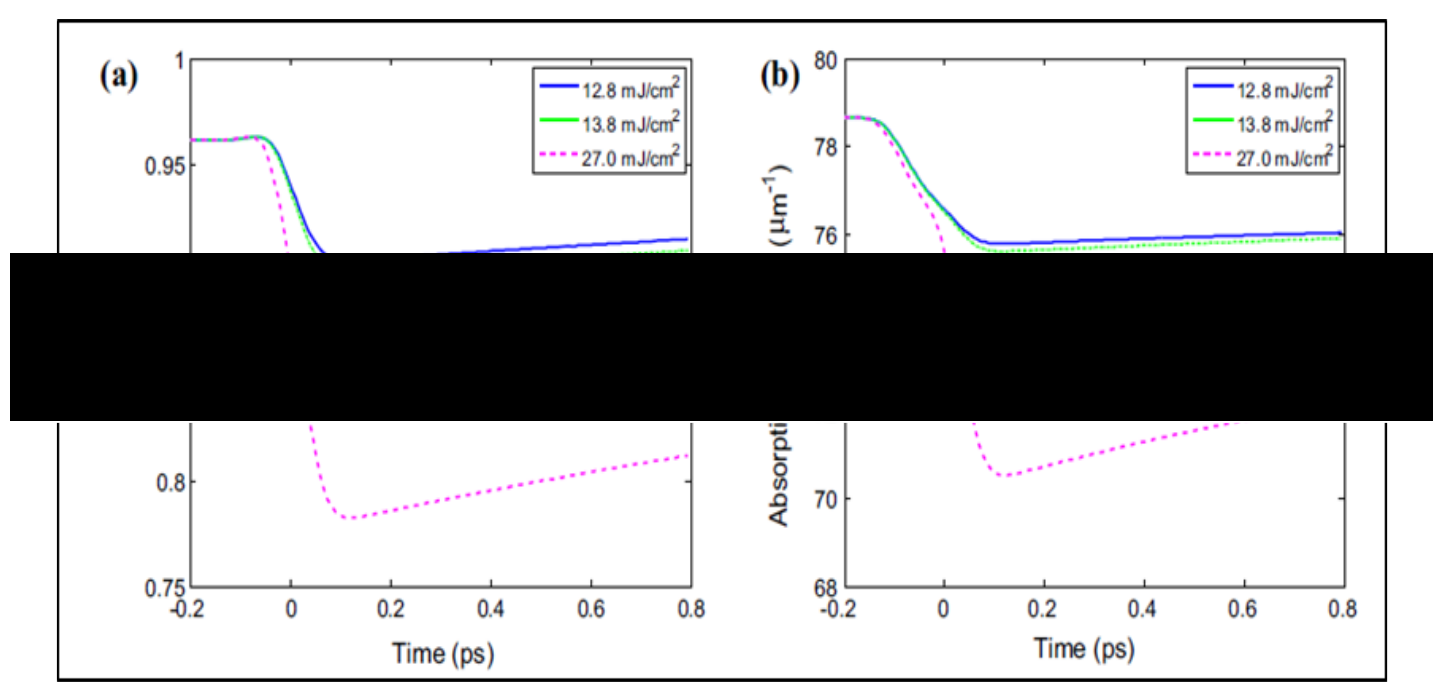

Figure 1.23 Time dependence of the calculated surface reflectivity (a) and absorption coefficient (b) for a single-shot laser pulse [100]

The main outcome of this study was simulating the ultrafast laser heating process to determine the laser energy required to achieve the most suitable temperature to sinter copper ink, which requires further investigation [100]. 


\subsection{Summary}

Based on the literature review, printing and sintering copper nano- or micro-particles is a complex topic, and it is also clear that multiple factors affect the printing methods, the sintering process and the substrate used, which needs to be taken into account.

One of the main factors is the ink properties; the ink of mixed particle sizes could be a promising bonding material to achieve better adhesion, better conductivity [52], and better stability [3]. Therefore, three different inks will be investigated: pure Nano copper, 50\% Nano 50\% Micro, and 20\% Nano 80\% Micro. Another factor is the sintering temperatures, which can be affected by the substrate utilized (glass, polymer). In the literature, the sintering temperature applied was $100^{\circ} \mathrm{C}-300^{\circ} \mathrm{C}$, which has been found to be the most influential temperature to remove the solvent from the ink after $60 \mathrm{~min}$ [101]. There is also the influence of the sintering atmosphere, which affects the oxidation behaviour. According to the literature, it tends to be subjective and material-dependent; however, the mixed gas atmosphere is the most promising sintering environment [76].

It was found that the tube furnace reducing atmosphere was the most practical to operate a reducing atmosphere [58]. The literature review shows that there are many better sintering methods; however, they are more operating intensive and require specialised equipment, and photonic sintering is widely applied [102] [103] [104] [105]. The main finding of the literature review is that the high photonic energy can be useful at a determined level if the ink was printed onto a less heat-sensitive substrate such as glass [82]. Laser sintering was considered as a robust method that would damage the thin film with a limitation application taking the employed substrate into account. However, it is necessary to investigate the use of a low-power laser with a solid substrate. It appears that there are few or no studies on processes which work on the thick film that has been photonically sintered, which is the film deposited by screen printing processes. 


\section{Chapter 2. Materials and Experimental Methods}

\subsection{Introduction}

This chapter presents the description of the methods, materials and equipment employed in this project. It also provides the main information on the operational principle of the manufacturing and characterization equipment, as well as the justification for its use. This chapter ends with a detailed explanation of the validity and reliability of all the experiments and analysis equipment, which leads to the results chapter.

\subsection{Selection and preparation of materials}

Nano- and micro-copper printing inks were supplied by Intrinsiq Materials Ltd (UK). Intrinsiq Materials was a recognized developer in making nano materials and producing conductive inks and pastes for research and for commercial electronic sectors. In this study, 100N, 50N50M and 20N80M nano-copper-based inks were utilized with several sintering processes (Table 2.1). These inks were blended by Intrinsiq via manipulation of the manufacturing process, in which the particle size distribution is varied by altering the argon flow rate, temperature, and copper melt temperature into their reactor. This manufacturing information is proprietary and was not shared. What is known is that the larger particle size is easier and cheaper to manufacture, as the particle size tolerances are significantly wider, separation of the particles from the gas stream is easier, and argon gas flows are lower. There is, therefore, a commercial driver in using the micro-particles in ink.

Table 2.1 Copper inks examined in the study (visually sized by SEM)

\begin{tabular}{|l|c|c|c|c|c|}
\hline Notation & $\begin{array}{c}\text { Intrinsiq } \\
\text { product } \\
\text { code }\end{array}$ & $\begin{array}{c}\text { Mass } \\
\text { available }(\mathrm{g})\end{array}$ & $\begin{array}{c}\text { Nominal } \\
\text { nano:micro }\end{array}$ & $\begin{array}{c}\text { Micro } \\
\text { particle } \\
\text { size }(\mu \mathrm{m})\end{array}$ & $\begin{array}{c}\text { Nano } \\
\text { particle } \\
\text { size }(\mathrm{nm})\end{array}$ \\
\hline $100 \mathrm{~N}$ & CP007 & 76.2 & N/A & N/A & $30-50$ \\
\hline $50 \mathrm{~N} 50 \mathrm{M}$ & IMCE4101 & 72.6 & $50: 50$ & $1.5-2.5$ & $30-50$ \\
\hline 20N80M & IMCE4118 & 79.2 & $20: 80$ & $2.0-2.5$ & $30-50$ \\
\hline
\end{tabular}


During the course of the PhD study, Intrinsiq Materials ceased trading in the UK, and therefore further information on its blending process was not available. More importantly, the volume of material that was available was limited. This issue became a significant research challenge, as potential avenues of investigation were curtailed by material availability. Each printing trial required a minimum of $250 \mathrm{~g}$ of material for the correct operation of the printing press (section 2.2.4). Although the manufacturing assets of Intrinsiq Materials were shipped for installation in the US parent company, these were not operating within the timescales of the project, and much of the manufacturing knowledge was lost.

The inks tested were standard inks designed for reducing oven sintering. Intrinsiq also developed materials of which the formulations were tailored to photonic sintering. As laser sintering is similar to photonic sintering, a repeat study using this alternative formulation was also conducted. The characteristics of each of the materials were examined by employing several techniques, such as thermogravimetric analyser (TGA), particle size distribution (from SEM images of the printed film) and rheology, as this has been shown to impact the print characteristics [106].

\subsubsection{The substrate preparation}

The substrate used in all the methods was FTO-coated glass made from $3 \mathrm{~mm}$ thick NSG Tec 07. The FTO-coated glass is a standard product used in the architectural glass industry, where '07' corresponds to the sheet resistance of the CVD-coated FTO layer on the glass. This substrate was chosen, as it is the substrate of choice for lowcost third-generation PV utilizing Perovskite and OPV technologies, one of the target markets for printed copper.

A $300 \mathrm{~mm} \times 300 \mathrm{~mm}$ sheet was cut into 36 samples of $50 \mathrm{~mm} \times 50 \mathrm{~mm}$ (Figure 2.1). The samples were immersed in a DI water tank in a $40 \mathrm{kHz}$ sonication bath for $30 \mathrm{~min}$ at $80^{\circ} \mathrm{C}$ to remove debris and surface contaminates benefiting from the cavitation [107] and was subsequently cleaned with methanol then acetone to remove any organic material left on the surface. Samples were then dried by compressed nitrogen and kept in a vacuum bag prior to the printing process. A resistance measurement was taken before printing. 


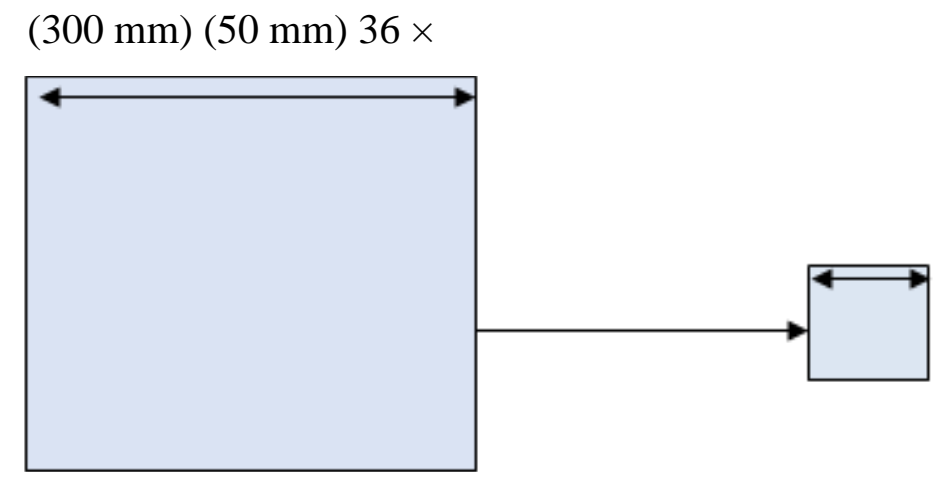

Figure 2.1 FTO glass sheet substrate cut into 36 samples of $50 \mathrm{~mm} \times 50$

$$
\mathrm{mm}
$$

\subsubsection{0kHz sonication bath utilized in substrate cleaning}

Ultrasonic baths are widely used in the cleaning process benefiting from the cavitation phenomenon [107]. The cavitation effect is generally well distributed through the liquid bath. Cavitation creates microbubbles during the acoustic cycles. These microbubbles grow during rarefaction cycles and can expand. However, they eventually reach an unstable size and produce smaller microbubbles that then collapse violently during the subsequent compression wave. Collapsing bubbles next to the substrate surface are subjected to micro jetting, which can remove any impurities on the surface [108].

\subsubsection{ATMA semi-automatic printer utilized in the screen printing}

Samples were screen printed by employing an ATMA semi-automatic printer (Figure 2.2), which was located in the SPECIFIC class 10,000 cleanroom in Baglan (Swansea University). A $250 \mathrm{~mm} \times 200 \mathrm{~mm}$ screen was used containing 110 threads/cm tensioned to $25 \mathrm{~N} / \mathrm{cm}$. The screen pattern was designed to enable three measurements of line resistance, sheet resistance and contact resistance to be obtained (Figure 2.2). The printer was set up for every trial with the parameters presented in Table 2.2.

Table 2.2 The print setting

\begin{tabular}{|l|l|}
\hline Squeegee angle & $70^{\circ}$ \\
\hline Squeegee speed $(\mathrm{mm} / \mathrm{sec})$ & 220 \\
\hline Flowcoat speed $(\mathrm{mm} / \mathrm{sec})$ & 220 \\
\hline Off contact distance & $1 \mathrm{~mm}$ \\
\hline
\end{tabular}


First, $50 \mathrm{~g}$ of the ink was placed on the screen, with this quantity being able to print around 50 samples. Each print was visually inspected to monitor the uniformity of the process. No variation in the prints could be observed within each printing session.

The resulting sample prints were dried for 30 min under normal air conditions at $60^{\circ} \mathrm{C}$ in a $3 \mathrm{~m}$ Thieme forced air dryer operating at a belt speed of $0.2 \mathrm{~m} / \mathrm{min}$, giving a total residence time of $15 \mathrm{~m}$. This dried the printed film through the removal of the solvent from the ink without reaching a temperature where nano-copper protective shells would be decomposed. Several sintering processes were carried out with all the materials used.

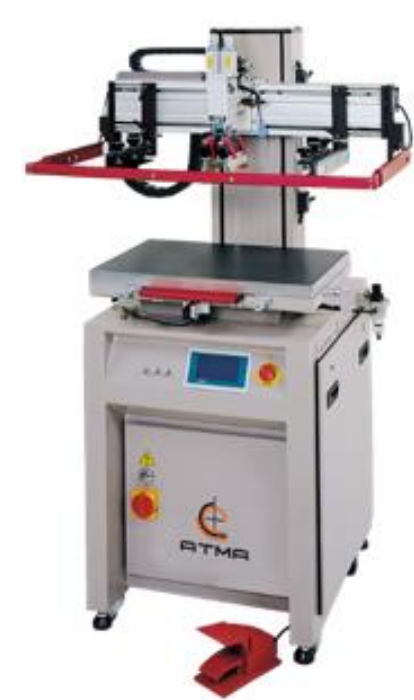

a

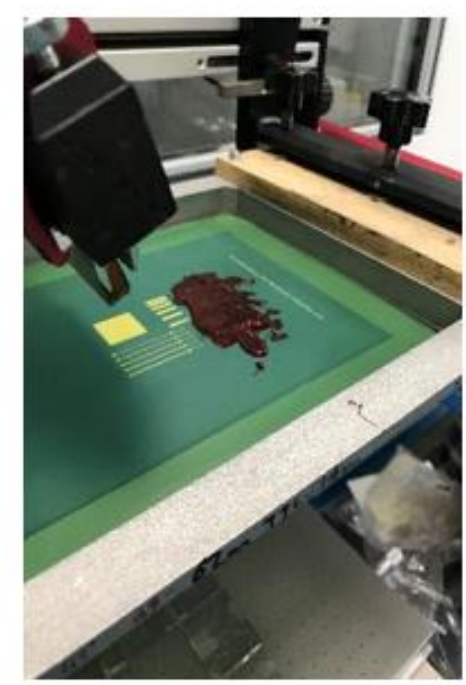

b

Figure 2.2 (a) ATMA AT-25PA/AT-45PA Digital Electric

Flat Screen Printer, (b) mesh and ink setting

Each ink was screen printed using an image (Figure 2.3) that consisted of areas which allowed for characterization of fine lined (300 $\mu \mathrm{m}-600 \mu \mathrm{m}$ wide) resistance, sheet resistance, and contact resistance. 


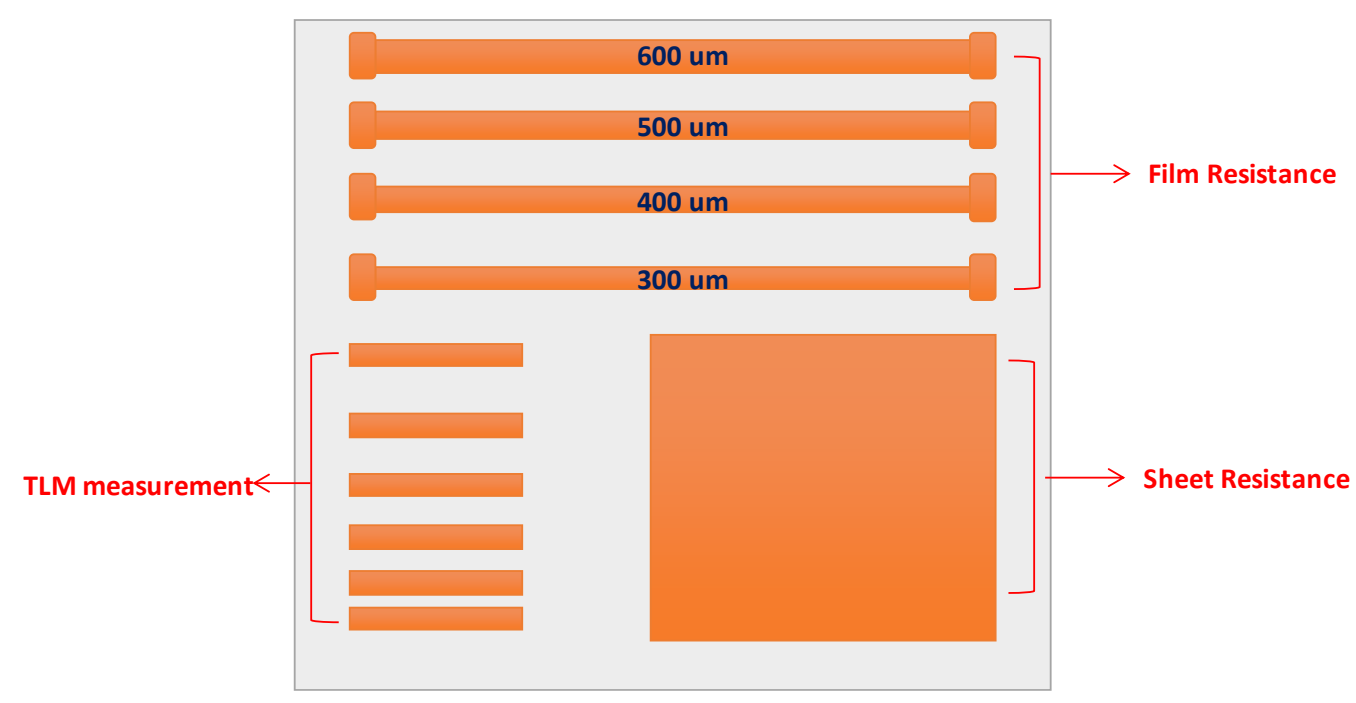

Figure 2.3 Screen pattern design

\subsection{Sintering method and equipment}

Samples were sintered by employing equipment with a variety of atmospheres. The thermal sintering using the controlled atmosphere oven was operating with three atmospheres: nitrogen, formic acid, and $5 \% \mathrm{H}_{2} / 95 \% \mathrm{~N}_{2}$. Photonic sintering by means of broad white light, laser and near infrared (NIR) sintering were also carried out.

\subsubsection{Thermal sintering using controlled atmosphere oven}

A Lenton model CSC 12/90/600H tube furnace, which can be sealed at either end and filled with the sintering atmosphere of choice, was used (Figure 2.4). The range of temperatures that can be applied using this oven is $45^{\circ} \mathrm{C}-900^{\circ} \mathrm{C}$. The tube had an internal diameter of $75 \mathrm{~mm}$ and a length of $1050 \mathrm{~mm}$, giving an internal volume of 46 $\mathrm{mm}^{3}$. The thermal sintering in the controlled atmosphere oven employed three atmospheres $\left(\mathrm{N}_{2}, \mathrm{~N}_{2}\right.$ travelling through formic acid, and a mix of $\left.5 \% \mathrm{H}_{2} / 95 \% \mathrm{~N}_{2}\right)$. Samples were sintered at temperatures of $175^{\circ} \mathrm{C}, 200^{\circ} \mathrm{C}, 225^{\circ} \mathrm{C}, 250^{\circ} \mathrm{C}$ and $275^{\circ} \mathrm{C}$ with time scales of $30 \mathrm{~min}, 60 \mathrm{~min}, 90 \mathrm{~min}$, and $120 \mathrm{~min}$. 


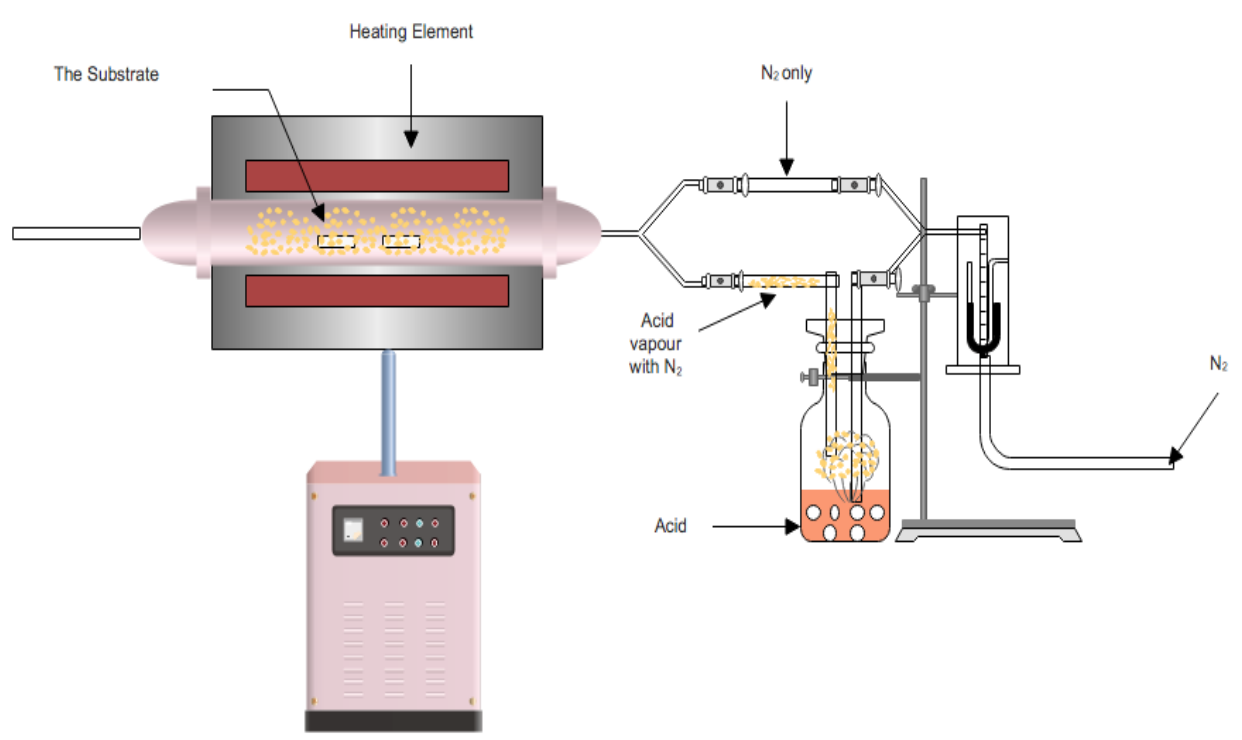

Figure 2.4 Controlled atmosphere oven

The controlled atmosphere oven was designed to allow only one gas environment through at a time. Three samples were sintered in each run, and all the experiments started at room temperature and then gradually increased to the required temperature. The gas flow rate was $4 \mathrm{~L} / \mathrm{min}$, which results in a complete theoretical change of gas within the oven every $4.5 \mathrm{~min}$.

The relevant gas flow came from $\mathrm{N}_{2}$ and $\mathrm{H}_{2} / \mathrm{N}_{2}$ premixed cylinders and was started prior to the temperature ramp, which took approximately $5 \mathrm{~min}$ to reach $200^{\circ} \mathrm{C}$. In the case of formic acid-induced reducing conditions, $\mathrm{N}_{2}$ had initially flowed through the oven before a switch at $200^{\circ} \mathrm{C}$, where the temperature had reached a sufficient level to begin formic acid thermal decomposition. Each sample was, therefore, not subjected to any oxidative atmosphere at temperatures above room temperature.

A 3-4 h wait was applied between each sintering condition to allow the oven to cool down. After sintering, samples were placed in a safe place and left to cool down to allow further measurements and analysis. 


\subsubsection{Broad-spectrum white light photonic sintering}

The broad-spectrum white light sintering employed the PulseForge 1200 photonic curing system supplied by NovaCentrix. The PulseForge 1200 was developed commercially to provide a configurable laboratory tool that could be used within a research environment to investigate and optimize photonic sintering such that substrate/ink combinations can be rapidly established. The lamps utilized in PulseForge 1200 can process a wide area at the same time without burning or affecting the substrate, and can deliver significant energy to the surface of the application that is sufficient to sinter metal ink [109] [110]. The generation of light energy depends on the size of the capacitors and the voltage applied to the capacitor. By connecting the capacitor to a voltage source, the two plates will develop equal and opposite charge. If the capacitor is then connected across the lamps, all the extra electrons in the negatively charged plate will travel to the positively charged plate through those lamps, which is called discharge [111]. The applied voltage is the primary control parameter that then dictates the resulting energy, as charging the capacitor to a higher voltage will cause a longer lighting pulse when the capacitor discharges. Therefore, the pulse length is in a positive relationship with the voltage applied [112]. The number of the pulses can be considered as the second control parameter to dictate the energy, and multiple light pulses can be applied, with the total energy output of each pulse being determined by the voltage and duration time employed.

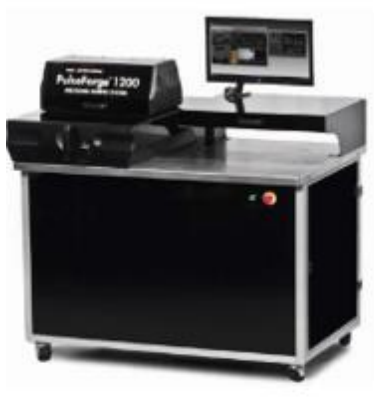

(a)

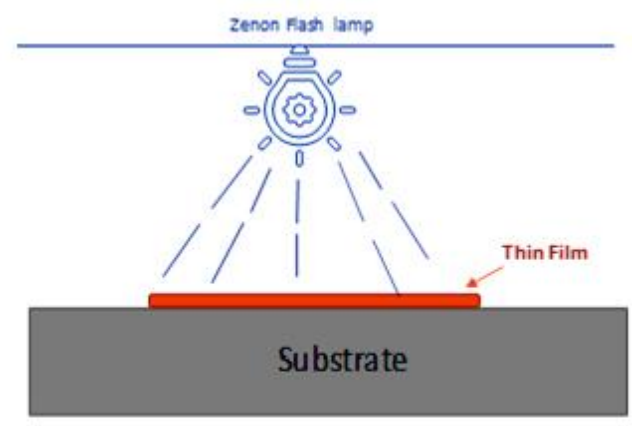

(b)

Figure 2.5 (a) Photograph of the PulseForge 1200, (b) schematic

The fast processing and the delivered energy on the substrate allow the sintering of copper inks $(100 \mathrm{~N}, 50 \mathrm{~N} 50 \mathrm{M}, 20 \mathrm{~N} 80 \mathrm{M})$ as a low-cost alternative to silver material [77]. Photonic sintering is also considered as a thermal sintering process, in which the 
thick deposited metal film is heated by employing pulsed light from a flash lamp. However, the exposure time is minimal $(\sim 1 \mathrm{~ms})$, which leads to a sintering process without heating the substrate [113]. Each sample was placed in the middle of the sintering bed (approximately $250 \times 250 \mathrm{~cm}$ ) so that the illumination was constant each time. The charging of the capacitor and the cooling of the bed meant that samples were sintered at intervals of around $3 \mathrm{~min}$. Given the variety of settings in the software control, an initial trial and error approach was adopted to find a starting point for a systematic study.

\subsubsection{Laser curing system}

A Rofin power line E $1064 \mathrm{~nm}$ laser (Figure 2.6) was employed to investigate laser sintering. It could be operated at frequencies of $10 \mathrm{kHz}-5000 \mathrm{kHz}$ and total power outputs of $10 \mathrm{~W}-40 \mathrm{~W}$. The laser was mounted on the coil line in the SPECFIC pilot line in Baglan and was designed to laser mark over a $300 \mathrm{~mm}$ wide substrate passing under the beam. The beam initially passes through an expander to reach a beam diameter of $50 \mu \mathrm{m}$. It is then reflected onto the substrate over a distance of approximately $2 \mathrm{~m}$ by a mirror, of which the angle is varied automatically by the software to allow the beam to scan over the surface. The overall scanning area is 200 $\mathrm{mm} \times 300 \mathrm{~mm}$, and each $50 \mathrm{~mm} \times 50 \mathrm{~mm}$ sample was placed in the middle of this area such that beam intensity due to non-normal targeting was minimized.

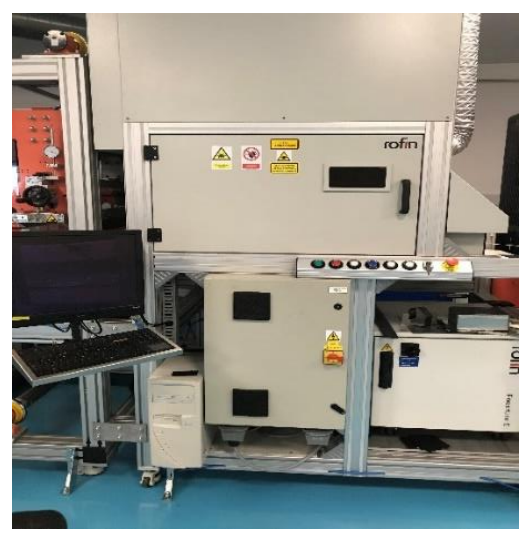

Figure 2.6 Rofin laser system

The laser system comes with Visual Laser Marker, which allows the required setting to be sent from the PC to the laser machine. 
The laser produced a direct conversion of electrical current into laser radiation, as the laser emitters are aligned by a diode laser bar. In simple terms, a laser-generated laser diode consists of a tube filled with atoms that called overactive medium. When the energy is applied to these atoms, they will move to the active state, and with the reflections, the mirror is placed at the ends of the tube to confine the photons to the tunnel, as one of the mirrors is made reflectance. The other mirror is made slightly transparent to allow part of the laser to escape [114].

\subsubsection{NIR sintering}

NIR sintering can be considered as a photonic sintering process. The ink was sintered by NIR irradiation at room temperature and in ambient conditions. The NIR system (HERAEUS), which was supplied by Thermal Processing GmbH, consists of six twin tube NIR lamps (Figure 2.7), a power controller, and an air-cooling module (Heraeus Co. Ltd). The maximum electrical power density of the system is $250 \mathrm{~kW} \mathrm{~m} \mathrm{~m}^{2}$. In this study, a minimum sintering time of $20 \mathrm{~s}$ was set up, as a stable time was needed for NIR light irradiation. The samples were placed at a distance of $50 \mathrm{~cm}$ from the lamps. The applied power and on-time were adjusted to change the sintering conditions, and the power settings utilized were $20 \%-100 \%$ with a variety of sintering periods (20-60 s).

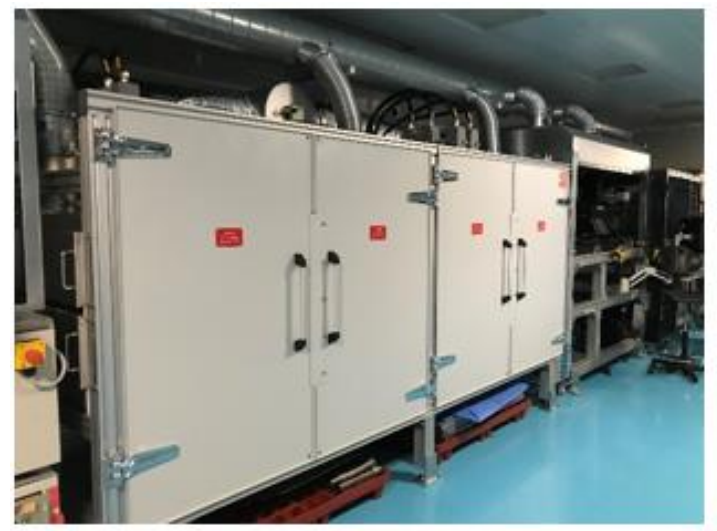

(a)

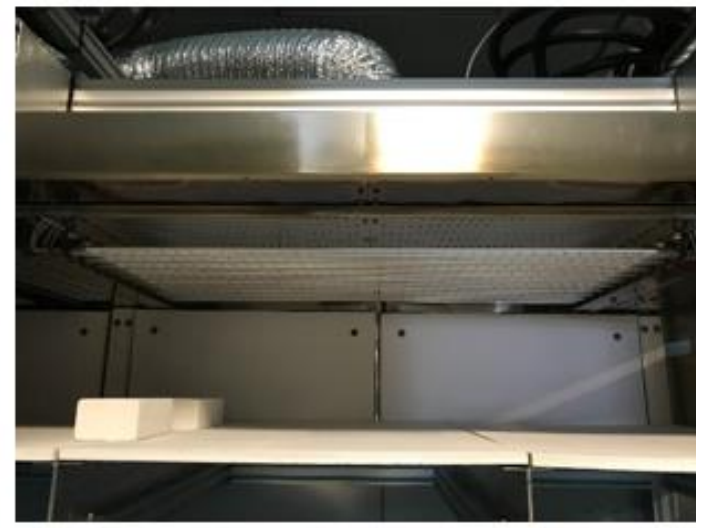

(b)

Figure 2.7 (a) NIR unit, (b) NIR lamps 


\subsection{Measurement and characterization equipment}

Measurement and characterization equipment is used to define the specific material characteristics of the obtained samples. To be able to better interpret the results, the measurements require specialized equipment for surface and bulk investigation. The equipment used is discussed in the following sections.

\subsubsection{Brookfield rheometer}

Rotational viscometers were utilized for measuring the ink viscosity. Viscosity is the material's resistance to flow and considered a primary property, as the material's flow has a significant impact on the printing process [115]. The cone-and-plate geometry was used in this device, measuring the gap between the rotating surface and sample plate and the spindle. Thus, shear rate and shear stress values can be calculated by applying Equation 2.1, Equation 2.2 and Equation 2.3. Figure 2.8 illustrates the physics of the viscosity measurement [116].

$$
\begin{aligned}
\text { Shear Stress }\left(\frac{\text { dynes }}{c m^{2}}\right) & =\frac{\tau}{\frac{2}{3 \pi r^{3}}} \\
\text { Shear Rate }\left(\operatorname{Sec}^{-1}\right) & =\frac{\omega}{\operatorname{Sin} \theta}
\end{aligned}
$$

Viscosity $\left(\mathrm{mPa}^{*} \mathrm{~s}\right)=\frac{\text { Shear stress } x 100}{\text { Shear Rate }}$

where

$\tau=\%$ Full Scale Torque $($ dyne $-\mathrm{cm})$

$r=$ Cone Radius $(\mathrm{cm})$

$\omega=$ Cone $\operatorname{speed}(\mathrm{rad} / \mathrm{sec})$

$\theta=$ Cone Angle(degree) 


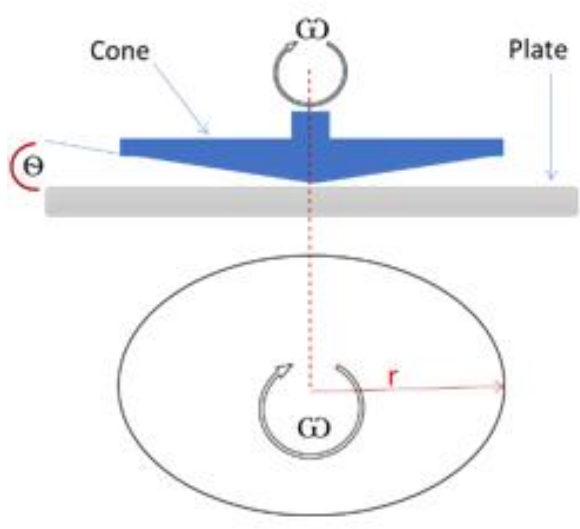

(a)

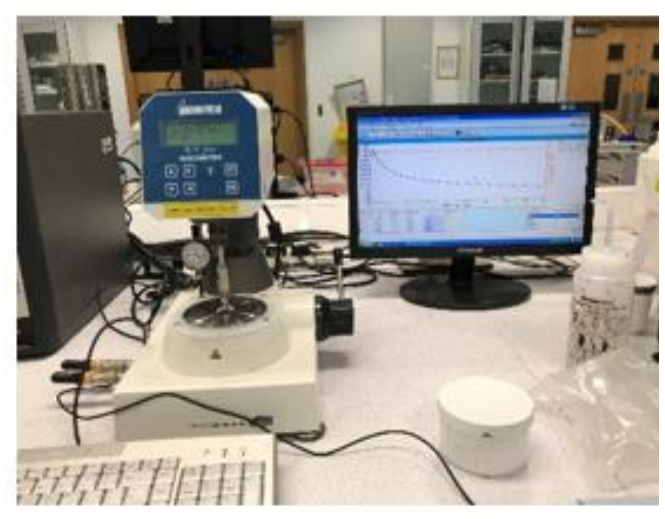

(b)

Figure 2.8 (a) Schematic, (b) photograph of the Brookfield rheometer

\subsubsection{Thermogravimetric analyser (TGA)}

TGA is a technique used to characterize material by measuring the mass change as a function of temperature or time [117]. The factors that can be measured by TGA (Figure 2.9) are the composition, purity, decomposition reaction, absorbed moisture contents, and decomposition temperature.

TGA measures the mass of the sample as it is heated in a defined atmosphere. When the reactants are heated, they produce some products and gas, and the type of change applied to the test material involves a loss in mass or weight. The loss is attributed to the gas, which is obtained by decomposition of reactants [118]. Afterwards, a typical mass loss curve resulted. The curve with Y mass loss and X temperature or time shows the loss of any volatile components, such as moisture and solvent. The subsequent curve refers to the decomposition of the material. 


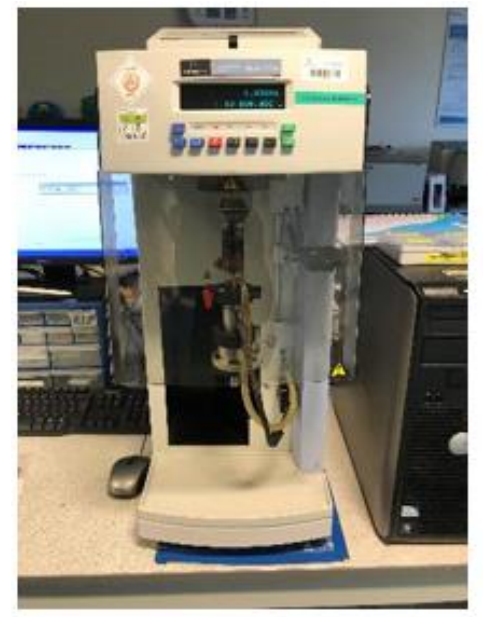

(a)

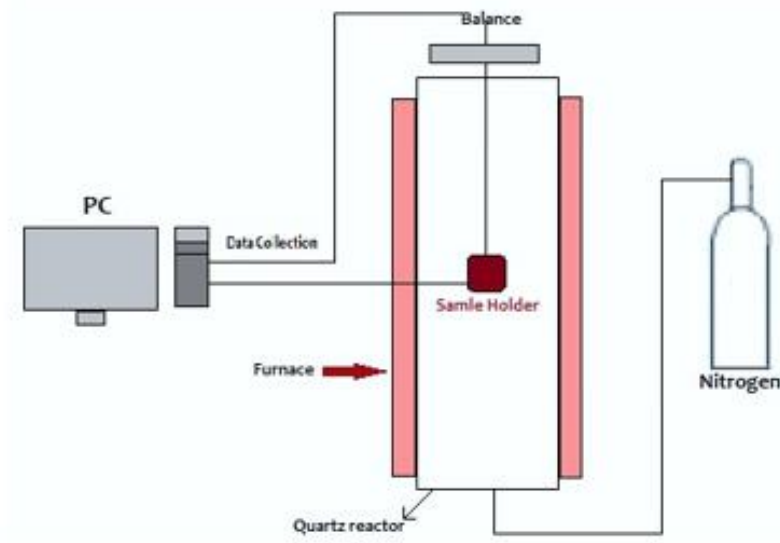

(b)

Figure 2.9 (a) Photograph of the TGA, (b) schematic

The samples and the sintering plan help to identify the approximate temperature of the material's transition. A scan was carried out at $25^{\circ} \mathrm{C}$ per min, beginning and ending at $350^{\circ} \mathrm{C}$ below and above the transition of interest of the materials. The scan was reviewed to adjust the beginning and ending temperatures accordingly.

\subsubsection{Colour spectrophotometer}

The colour of the samples was examined using a SP64 Sphere spectrophotometer operating with an integrating sphere. The measured colour was analysed using the CIE-L*a*b* system, which is a colour space defined by the International Commission on Illumination (CIE). The colour is expressed as three numerical values ( $\mathrm{L}^{*}$ for the lightness, $\mathrm{a}^{*}$ for the green-red components, and $\mathrm{b}^{*}$ for the blue-yellow components). The CIE-L*a*b* system was designed to be perceptually uniform concerning human colour vision, meaning that the same amount of numerical changes in these values corresponds to about the same amount of visually perceived change [119]. The total difference in colour $(\Delta E)$ can be identified using Equation 2.4.

$$
\Delta E^{*} a b=\sqrt{\left(L_{2}-L_{1}\right)^{2}+\left(a_{2}-a_{1}\right)^{2}+\left(b_{2}-b_{1}\right)^{2}}
$$

where the subscripts 1 and 2 refer to samples 1 and 2, respectively. 


\subsubsection{Line resistance characterization}

The resistance measurement of the printed structures was carried out using a Megger MIT 330-EN. The Megger MIT (Figure 2.10) performs a basic resistance measurement test with accuracy of $+/-3 \%$.

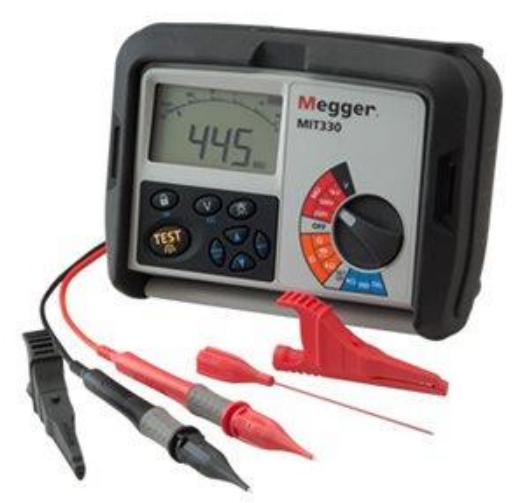

Figure 2.10 Megger MIT 330

The Megger MIT 330 was utilized in the measurement of the line resistance and the transmission line measurement (TLM). The line resistance can be explained as the current flowing through the printed line film is like a fluid flowing through a pipe, and the voltage drop across the film is like the pressure drop that pushes the fluid through the pipeline. Therefore, the resistance of a conductive line depends on its shape and the material of which it is composed [120] [121] [122]. The copper line was printed in a rectangle shape (Figure 2.11). The absolute line resistivity was also calculated after measuring the film thickness by employing a Taylor Hobson S-28 profilometer, following which the resistivity can be analysed using Equation 2.5 and Equation 2.6.

$$
R=\rho \frac{L}{A} \Omega
$$

$$
\rho=\frac{R \times A}{L}=\frac{\Omega \times m^{2}}{m}=\Omega . m
$$

where

$$
R=\text { Resistance } \rho=\text { Resistivity } L=\text { Length } A=\text { Area }
$$




\section{$\mathrm{L}=$ Length}
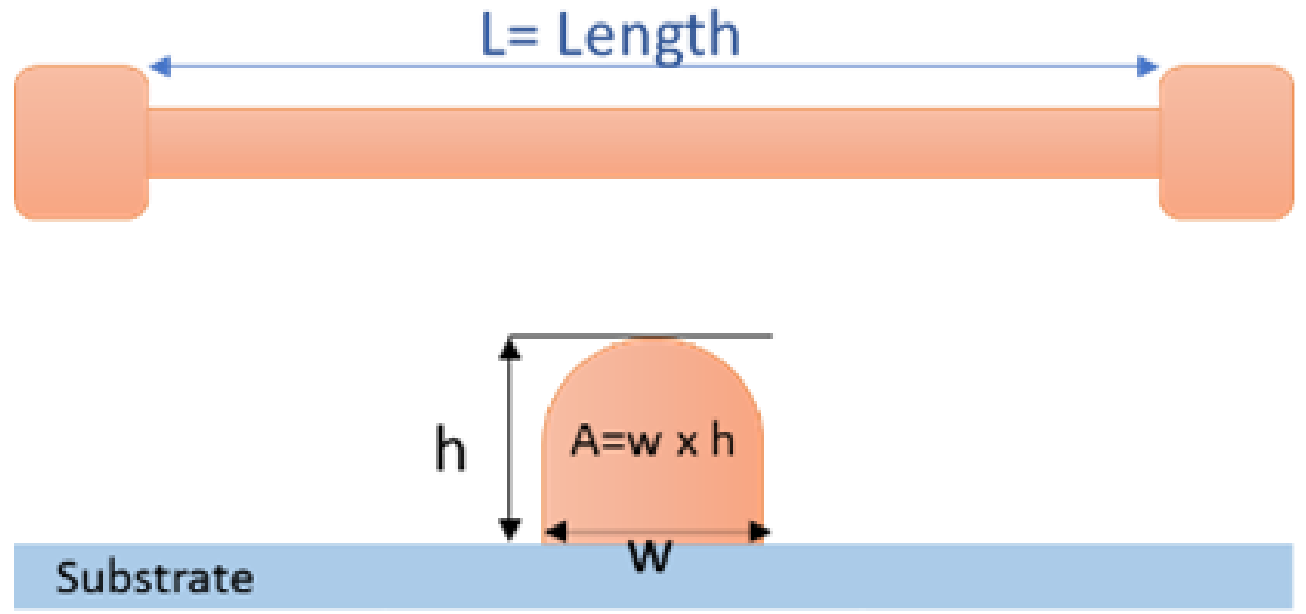

Film Cross sectional Area

Figure 2.11 Printed line diagram

TLM is a technique employed to determine the contact resistance between the printed thin film and the FTO substrate. This measurement requires printing a series of metal film contacts separated by various distances $(1 \mathrm{~cm}-5 \mathrm{~cm})$. Probes of the multi-meter are applied to pairs of film contacts, and the resistance between them is measured by using a voltage across the contacts and measuring the resulting current [123]. The contact resistance refers to the total resistance contribution of the printed film, which can be attributed to the contacting interfaces of the FTO glass surface and connections as opposed to the copper film resistance. The measurement was carried out on each printed contact; the resistance was obtained from the slope of the curve in Figure 2.13. The resistance was then plotted as a function of the contact spacing $\mathrm{d}$. 


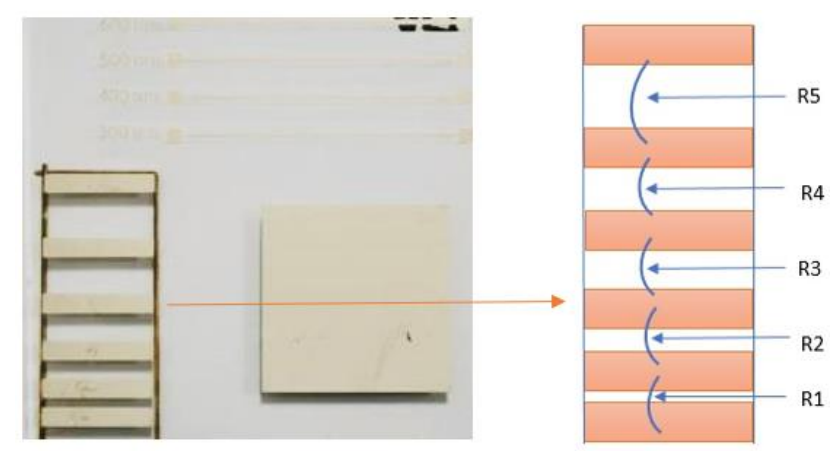

Figure 2.12 Plot to extract transfer length from the TLM method

The TLM printed pattern was disconnected from the other part of the substrate by applying a high-power laser (40 W with $100 \mathrm{kHz}$ ) before measuring the resistance between two grids, as illustrated in Figure 2.12. The contact resistance was obtained from the plot in Figure 2.13 using the extracted transfer length (LT). The sheet resistance (Rs) contact resistance (Rc) and width of the contact pattern $\mathrm{W}$ for the total resistance (Rt) of the TLM structures can be extracted from the plot and by Equation 2.7 .

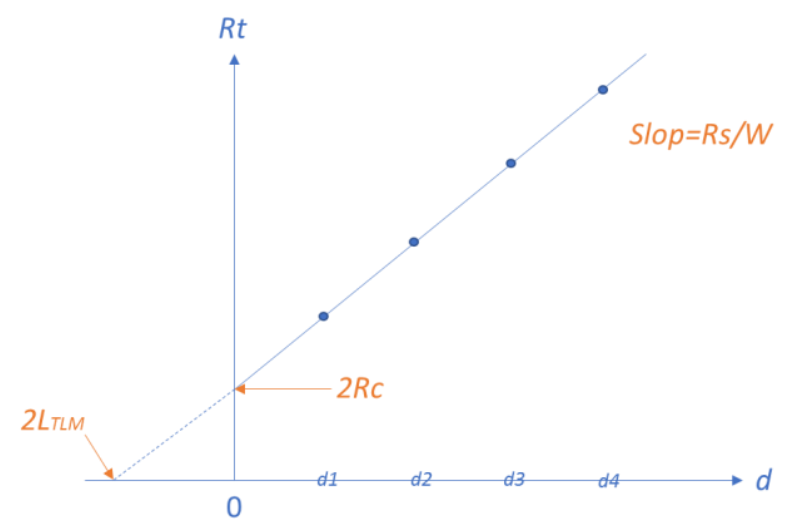

Figure 2.13: Plot to extract transfer length from the TLM method

$$
R T=2 R C+\left(\frac{R_{S}}{W}\right) d
$$

where

$R s=$ sheet resistance

$R c=$ contact resistance

$R t=$ total resistance 


\subsubsection{Four-point sheet resistance measurement}

A Jandel CYL-RM3000 Four Point Probe was employed to obtain the printed film sheet resistance using a four-point probe measurement. The four-point probe measurement needs a specific geometric shape of the printed film, and that was planned on the screen mesh design to obtain a square shape of the film to enable this measurement. Describing the measurement principle for the four-point probe method as four pins with the distance of the same space (Figure 2.14) are applied to the surface of a sample, with the current flowing through the two outer pins [124]. When the difference in potential between the two inner probe pins is measured, the sheet resistance can be measured utilizing Equation 2.8.

$$
R s=\frac{\Delta V}{I} \frac{\pi}{\operatorname{In} 2}=\frac{\Delta V}{I} 4.5324[\Omega . s q]
$$

where

$\Delta V=$ the change in voltage measured between the inner probes

$I=$ current through the outer probes

$R s=$ sheet resistance $(\Omega / s q)$

units $\Omega / s q$, or 'ohms per square', to differentiate it from bulk resistance.

The RM3000 supplies constant currents between $10 \mathrm{~mA}$ and $99.99 \mathrm{~mA}$ and measures sheet resistance. The quoted range is from 1 milliohm-per-square $\left(10^{-3}\right)$ to $5 \times 10^{8}$ ohms-per-square with $0.3 \%$ accuracy. The method in Figure 2.14 can be applied to obtain a sheet resistance measurement from the screen-printed sample.

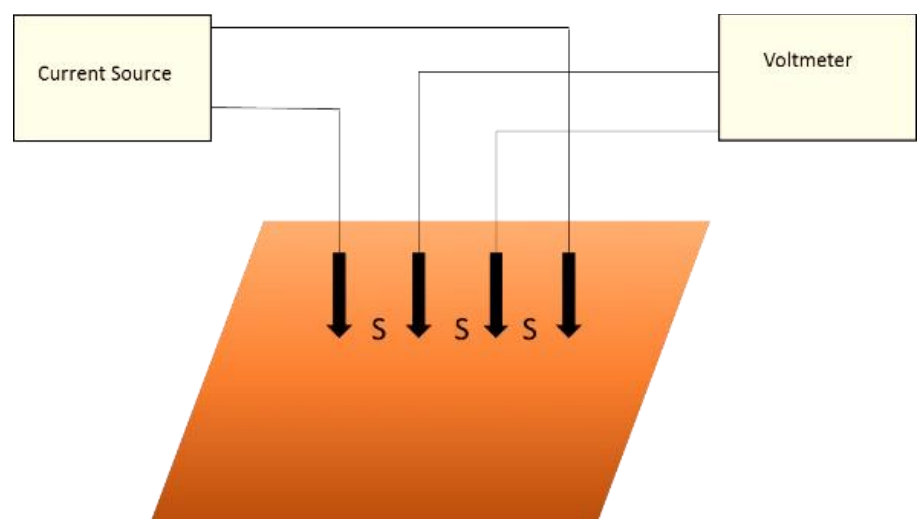

Figure 2.14 The four-point probe sheet resistance measurement 


\subsubsection{Film stability test}

The stability test of the printed film was carried out using the Megger MIT 330 by measuring the line resistance of 600 um every 7 days for four measurements. The reason for conducting the test was to investigate the lifetime of the printed copper printed structures. The samples were stored in a sealed plastic container and left in a laboratory cupboard unit in an air-conditioned laboratory. Ambient conditions in the laboratory were $20^{\circ} \mathrm{C}+/-1^{\circ} \mathrm{C}$ and $\mathrm{RH}$ of $60 \%+/-20 \%$.

\subsubsection{Crosscut adhesion test (TQC)}

The TQC test kit was used in this test to measure the adhesion. It is a stainless steel measuring tool that features a $1 \mathrm{~mm}, 1.5 \mathrm{~mm}, 2 \mathrm{~mm}$ and $3 \mathrm{~mm}$ crosscut adhesion test. The $1 \mathrm{~mm}$ crosscut was utilized in this study by employing fibre reinforced tape. The adhesion test methods include procedures for assessing the adhesion of the printed films to FTO substrates by applying and removing pressure-sensitive tape over cuts made in the film (Figure 2.15). A lattice pattern with six cuts in each direction is made in the film to the substrate, the pressure-sensitive tape is applied over the lattice and then removed, and adhesion is evaluated by comparison with descriptions and illustrations (Figure 2.16).

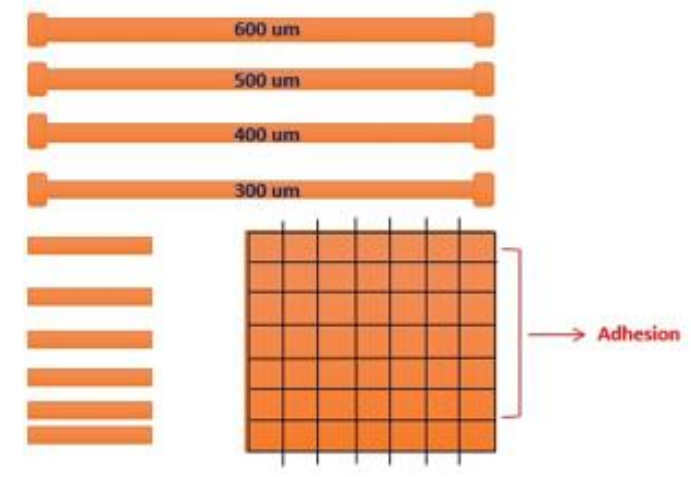

1

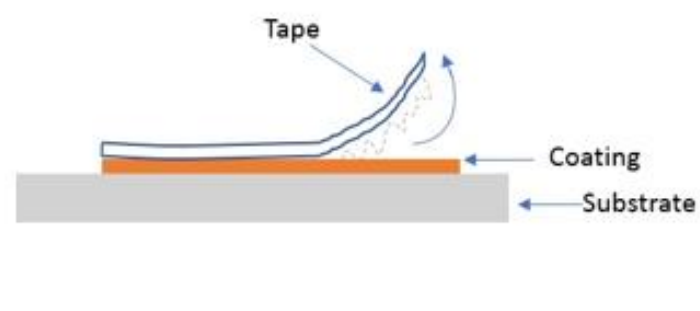

2

Figure 2.15 Lattice pattern with six cuts and peel profile 


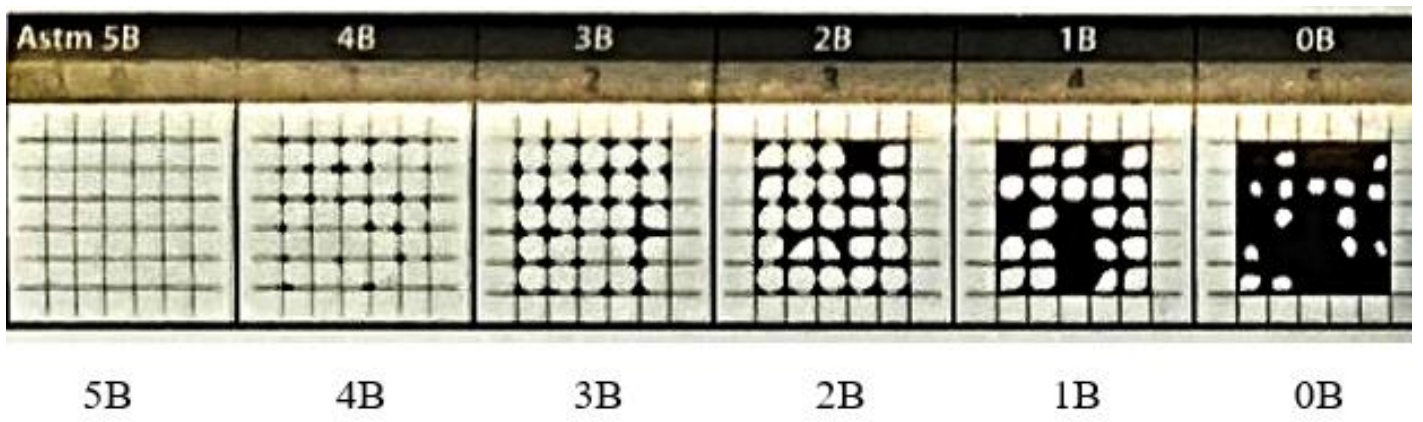

Figure 2.16 Classification of adhesion test (tape after the test)

\subsubsection{Scanning Electronic Microscope (SEM)}

An SEM was used as a surface examination method, as it enabled higher-resolution imagery than that obtained via optical means [125]. The main components of the microscope are (i) column for electrons beam generation, (ii) specimen chamber, (iii) vacuum pump and a control panel with a monitor. In the column to generate electrons, a V shape tungsten wire, also called a thermionic cathode, by applying electric current. It emits electrons into the vacuum, below the cathode a metallic disk with a central borehole installed called anode [126]. The anode is connected to a positive pole with a high-voltage source, and the thermionic cathode is connected to a negative pole. The strong electric field between the anode and the cathode accelerates the electrons downward. These electrons are called the primary electrons, the generated electrons that hit the specimen and the stage focused on the specimen surface by an electromagnetic lens located below the anode. Subsequently, the primary electrons remove electrons from the sample material; these removed electrons are called secondary electrons. In this stage, additional components are necessary, including electromagnetic deflection, raster scan generator, secondary electrons (SE) detector, and control panel with monitor [125]. The basic principle of an SEM is to detect the secondary electrons and use them to build up an image of the surface. To increase the number of detected electrons, a positively Boise grid is placed in front of the detector. The grid attracts the secondary electrons and leads them to the SE detector, and this is the way to generate a magnified image of the sample by recording the number of secondary electrons on the SE detector. Then the signal is amplified and displayed as a dot on the screen. After moving the electron beam to the next point on the sample, the measurement is repeated. The principle is that many recorded electrons lead to a 
bright image point, few lead to a grey image point, and none lead to a black point. Many image points are used in the scan to provide a natural-looking image of the specimen surface, and they can be seen on the monitor [127] [107].

Samples were examined with the SEM using several magnification options (100 times to 50,000 times) to show the best image of the substrate surface. After screen printing and a variety of sintering processes of the copper deposited on the surface, there was significant conductivity of the material, which then enabled the SEM analysis.

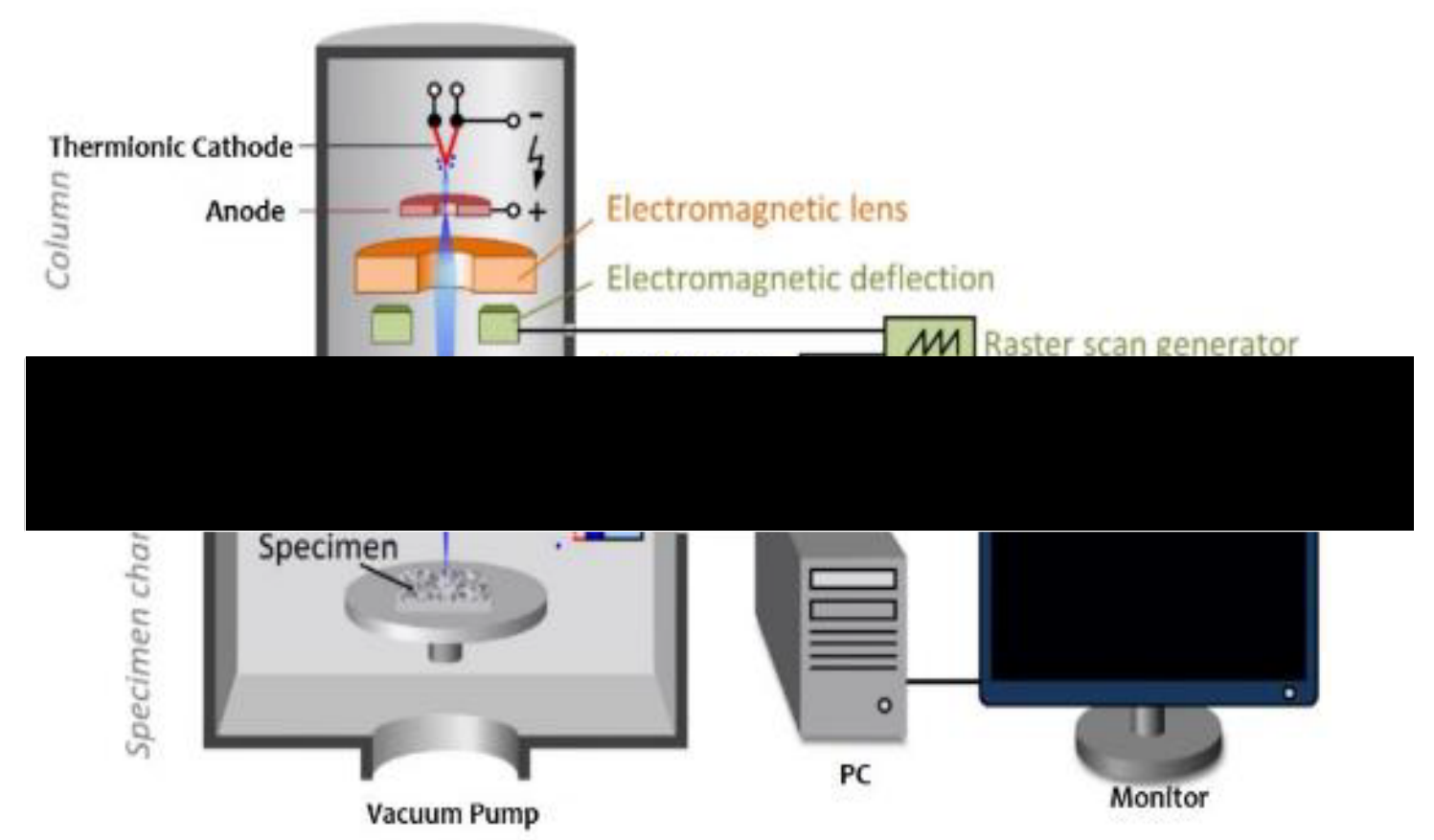

Figure 2.17 JEOL JSM-7800F SEM [128]

The images were taken at a magnification between 100 and 50,000 times on a JEOL JSM-7800 F SEM at the Bay campus in Swansea. The theoretical resolution of JEOL SEM is $0.8 \mathrm{~nm}$ at $15 \mathrm{kV}$. Therefore, it was likely that images could be taken for a nano-particle size of $30 \mathrm{~nm}-80 \mathrm{~nm}$. The grain structure size was graded as small indicates nano-particles a sintered (e.g. smooth surface). In contrast, large means a micro-particle that is less sintered, and the extreme change in surface morphology is induced by over-sintering. An extra electrons X-ray detector operating at a $10 \mathrm{kV}$ acceleration voltage can be used in this device to enable the energy dispersive X-ray spectroscopy (EDS) measurement for elemental mapping. 


\subsubsection{X-ray Diffraction (XRD)}

The Bruker D8 model was used to carry out XRD to examine the crystal strictures or thin-film materials. The minimum crystallite size is $20 \mathrm{~nm}$, for which a diffraction pattern can be collected by Bruker D8 XRD. The principle of operations is reliant on the production of X-rays in a vacuum tube [129] [130] [131]. Electrons created by passing current through a tungsten filament are accelerated and X-rays upon the electrons' impact with a copper target. The X-rays are targeted towards the sample, and their subsequent diffraction angle is measured by a detector (Figure 2.18).

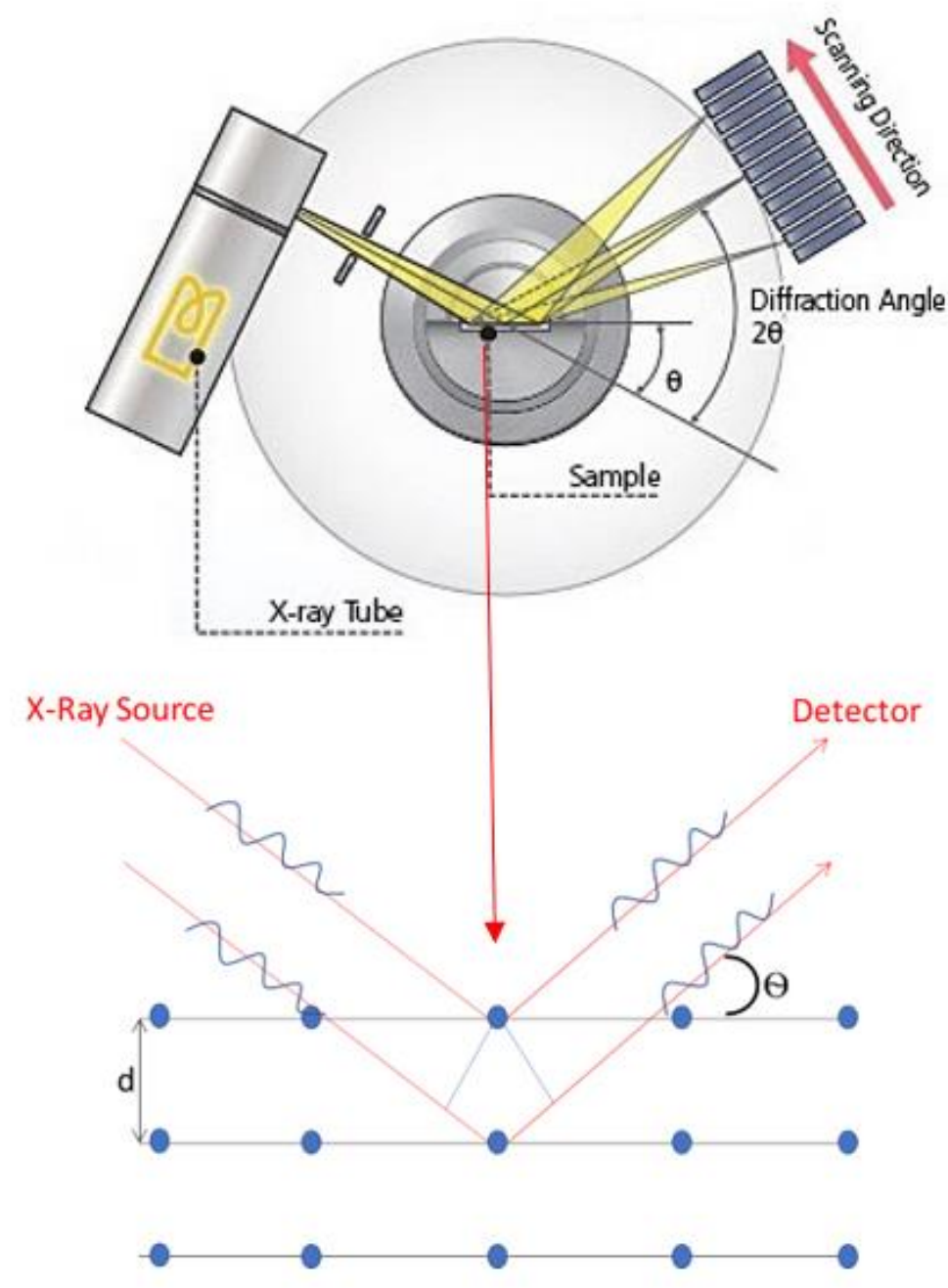

Figure 2.18 XRD diagram

X-ray has an angle theta reflected from the internal crystal separated by a specific distance (d) [132]. Thin-film materials or fine powder materials contain many crystals, 
and these crystals are located at random angles. Some of these crystals are oriented so that the X-ray beam, crystal and detector satisfy the Bragg equation.

$$
\mathrm{n} \lambda=2 \mathrm{~d} \sin \theta
$$

where $\lambda$ is the wavelength of the radiation employed; $d$ is the inter-planar spacing involved, or the distance between the crystals; $\theta$ is the angle between the incident or diffracted ray and the relevant crystal planes; and $n$ is an integer, referred to as the order of diffraction. This causes a signal spike at a specific detector angle and having an adequate number of crystals to have an even distribution at all possible crystal's orientation. During the scan, the detector rotates over a range of angles and detects the diffracted X-ray intensity such the peak diffracted angle represents the crystal spacing. 


\subsection{Error Estimation}

As with any process of manufacturing printed circuits and their characterization, there is a risk of possible errors linked to creation of the samples and their measurement. These can be split into those sources of variability which are present in the preparation of the samples and those which can be associated with measurement and characterization process.

Sample process variability is difficult to estimate and can be minimized by the adoption of best practice. The best practice developed during the initial stages of the experimental plan and the mitigation used to minimize the variability are summarized in Appendix A. Variability associated with the inherent accuracy of the characterization techniques was first examined by the selection of the correct measurement device and method and further by examining the accuracy of the device.

\subsection{Summary}

Chapter 2 has introduced the key sample manufacturing, sintering and characterization process, which were employed in the investigation described in this thesis. This has been carried out with the intention that the experimental procedure could be repeated if the same materials and equipment were available. Having described the processing and characterization process, the next chapter investigates the thermal sintering of the materials using the methodologies described here. 


\section{Chapter 3: Thermal sintering}

\section{Introduction}

The chapter introduces the results obtained by employing a reducing atmosphere during the thermal sintering process. A material characterization study was also carried out and presented in this chapter on wet and dry material to build a foundation sintering plan to be applied thermally according to the material's behaviour. Specifically, this chapter aimed to identify and understand the relationship and interactions between the final electrical performance of the printed film and the copper ink characteristics, sintering time, sintering temperature, and the atmosphere required for sintering.

\subsection{Materials Characterization of the wet ink}

Characteristics of the materials of the ink employed in terms of TGA and Brookfield. Rheology and the material composition ratio were measured using the methodology described in chapter 2.

\subsubsection{Thermogravimetric analysis}

Thermogravimetric analysis (TGA) was employed to investigate the thermal behaviour of the materials utilized by measuring the mass loss and investigating thermal stability. The operating conditions were a heating rate of $10{ }^{\circ} \mathrm{C} / \mathrm{min}$ and nitrogen atmosphere with a gas flow of $20 \mathrm{~mL} / \mathrm{min}$. The inks were investigated over a $25^{\circ} \mathrm{C}$ to $350^{\circ} \mathrm{C}$ temperature range.

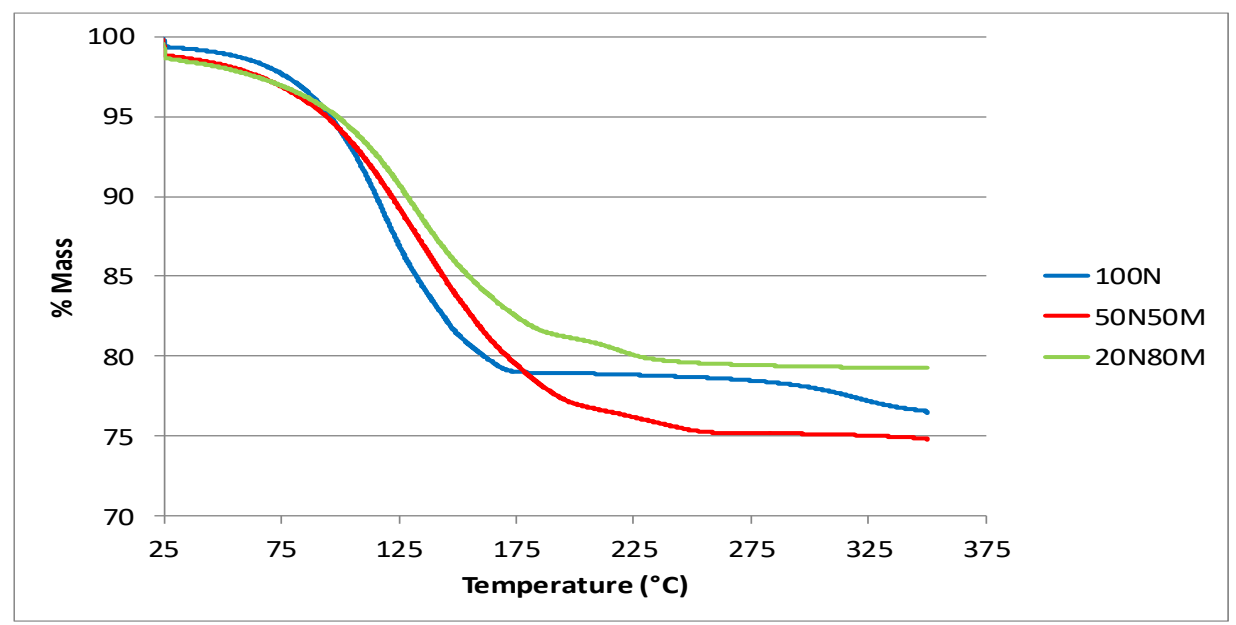

Figure 3. 1 TGA Thermal characteristics 


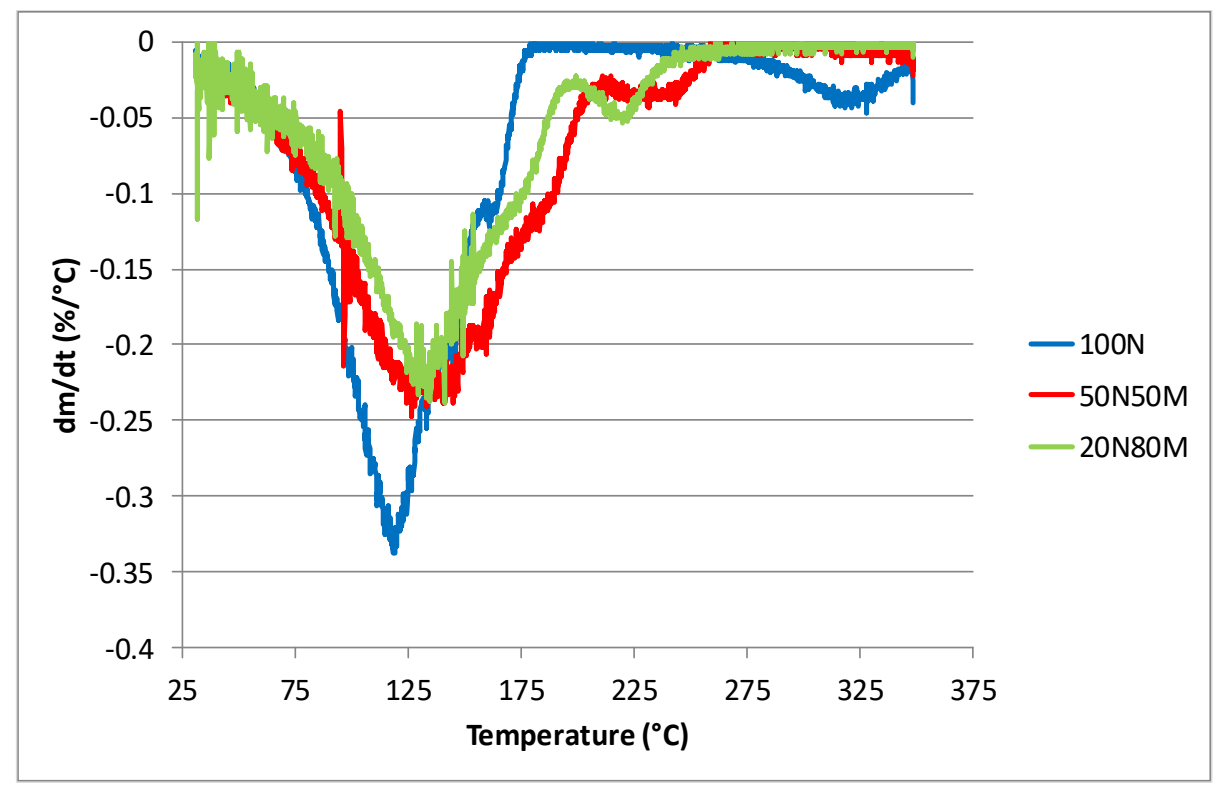

Figure 3. 2 Rate of Change of mass loss

Figure 3.1 and 3.2 show the thermal behaviour for the three materials utilized in this study. The mass loss occurs with the higher temperatures applied, at the range between $75^{\circ} \mathrm{C}-200^{\circ} \mathrm{C}$. The mass loss significantly achieved with 50N50M ink material. The greatest mass change was highly noticeable between the 20N80M and 50N50M, which could refer to a different chemical content such as the solvent amount and the particles size. However, the weight loss different started to increase at $95^{\circ} \mathrm{C}$ and above and this temperature assuming where no water or solvent left in the material what could be then attributed to a different amount of solvent utilizes on these materials. The solvent provides a stable suspension of the copper nano-particles, the higher solvent content in the $50 \mathrm{~N} 50 \mathrm{M}$ as shown by the TGA results in Figure 3.1 means that the solvent fills the inter-particle voids and is then lost during temperature raised. Another possible hypothesis is that it is a limitation of film compaction during drying due to collisions between the larger copper particles. The test also showed a slight deviation between both $100 \mathrm{~N}$ and $50 \mathrm{~N} 50 \mathrm{M}$ materials in the structure, as the mass loss was very similar at all temperature. Overall, all three inks exhibit similar thermal behaviour where there is a gradual reduction in mass at low temperature between $25-75^{\circ} \mathrm{C}$ due to the solvent evaporation, with a rapid decrease in the mass as the rate of solvent loss increases as it nears its boiling point, Table 3.1, Figure 3.2. 
Table 3. 1 Copper ink characteristics

\begin{tabular}{|c|c|c|c|}
\hline & $\mathbf{1 0 0 N}$ & $\mathbf{5 0 N 5 0 M}$ & $\mathbf{2 0 N 8 0 M}$ \\
\hline Particulate & Nano & Micro/nano & Micro/nano \\
\hline Micro: nano particle ratio & Nano only & $50 / 50$ & $80 / 20$ \\
\hline Primary solvent BP $\left({ }^{\circ} \mathrm{C}\right)$ & 117 & 136 & 136 \\
\hline
\end{tabular}

\subsection{2: The Viscosity measurement by Rheometer}

All three materials show similar rheological behaviour with near rest viscosities in excess of 100 Pas, followed by shear thinning behaviour towards a shear-thinning plateau approaching 30 Pas, Figure 3.3. As these are typical of screen printing inks [133], [134], and their similarity in the rheological profiles would suggest that any variations in deposit between characteristics are not attributed to changes in the deposition process.

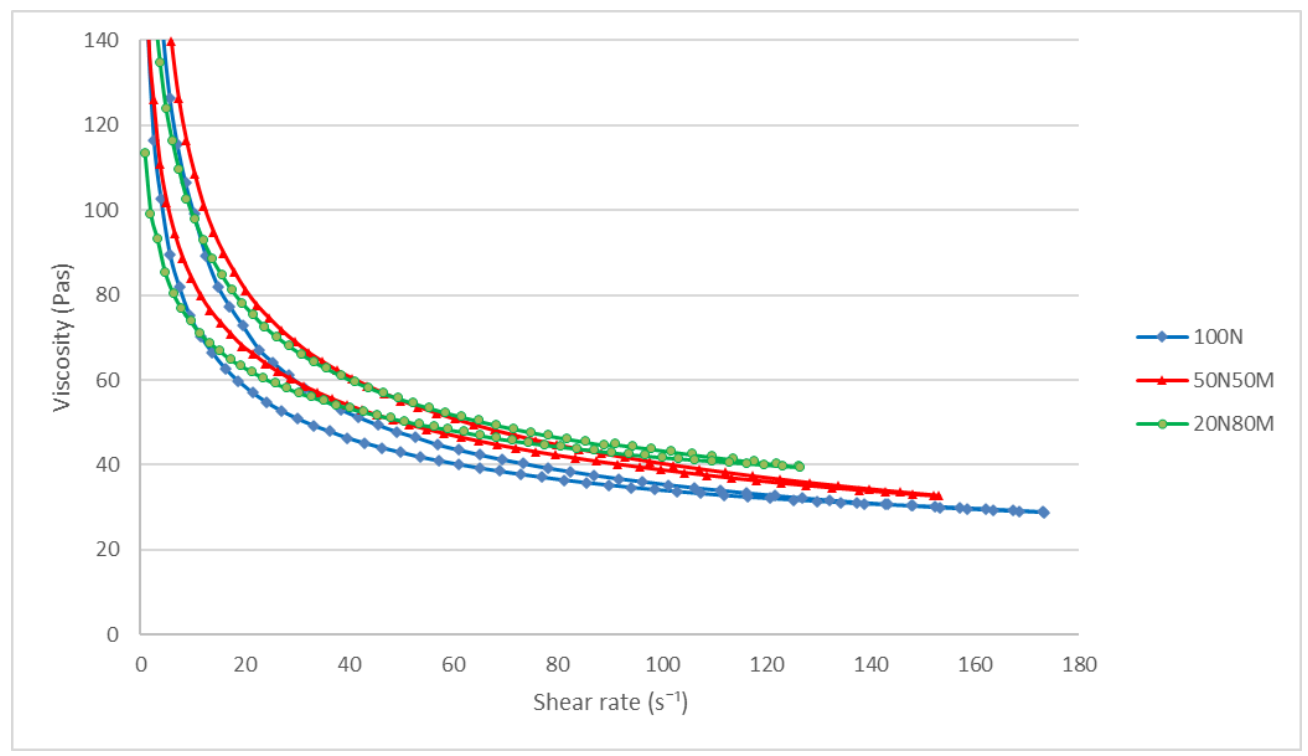

Figure 3. 3 Rheological characteristics of 3 materials over a 0 - 150 shear rate range. 


\section{2: Material characterization of the dry, un-sintered film}

Characteristics of the dry, un-sintered film employed in terms of the colour spectrophotometer, particle size distribution, (from SEM of the solid film). The colour difference was also tested and presented on employing spectrophotometer and optical microscope for visual optimization. Figure 3.4 shows the samples of the three materials after printing and drying.

\subsubsection{Visual characteristics}

The printed lines are straight at the edged and do not show any edge marking, which can be a common defect in screen printed lines, Figure 2.3. Each line is rendered well, and thus the assumption that there is little change in the print quality as a result of the minor rheological difference in the ink is valid. Each material is matt in colour and the microparticle inks have a brownish colour, Figure 3.4.

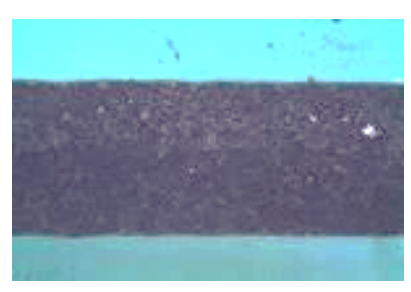

$100 \mathrm{~N}$

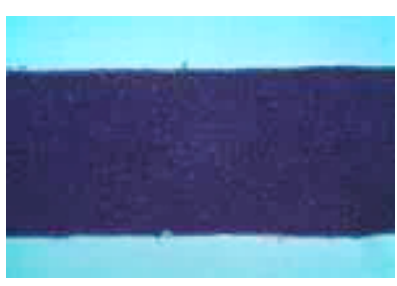

$50 \mathrm{~N} 50 \mathrm{M}$

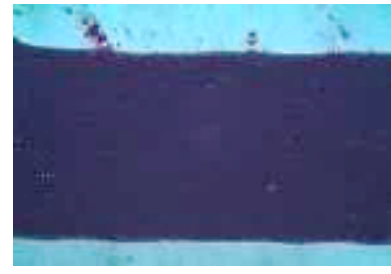

$20 \mathrm{~N} 80 \mathrm{M}$

Figure 3. 4 (10X) Dry screen-printed inks at $80^{\circ} \mathrm{C}$ for 30 minutes

\subsection{2: Spectrophotometer colour analysis}

Table 3.2 shows the Lab values using the methodology described in chapter 2, after drying for 30 minutes at $80^{\circ} \mathrm{C}$. The colour difference $(\Delta \mathrm{E})$ between the $50 \mathrm{~N} 50 \mathrm{M}$ and $20 \mathrm{~N} 80 \mathrm{M}$ materials was relatively small at 6.6 while the $20 \mathrm{~N} 80 \mathrm{M}$ and $100 \mathrm{~N}$ were 25.5 which reflects the impact of the nano-particles on the visual appearance which leads to a lighter and yellower printed film.

Table 3. 2 (L*a*b* absolute Colour)

\begin{tabular}{|c|c|c|c|}
\hline Ink & $\mathrm{L}$ & $\mathrm{a}$ & $\mathrm{b}$ \\
\hline $100 \mathrm{~N}$ & 41.4 & 19.63 & 34.23 \\
\hline $50 \mathrm{~N} 50 \mathrm{M}$ & 33.74 & 8.50 & 8.11 \\
\hline $20 \mathrm{~N} 80 \mathrm{M}$ & 39.49 & 10.82 & 10.31 \\
\hline
\end{tabular}




\subsection{3: SEM structure examination}

The dry materials were investigated using the SEM 10,000 magnification which showed a significant structure different. The SEM allowed a visual identifying of the ratio of the copper nano or micro-particles have been utilized in the preparation of these materials. The classification of these particles is highlighted in Figure 3.5.

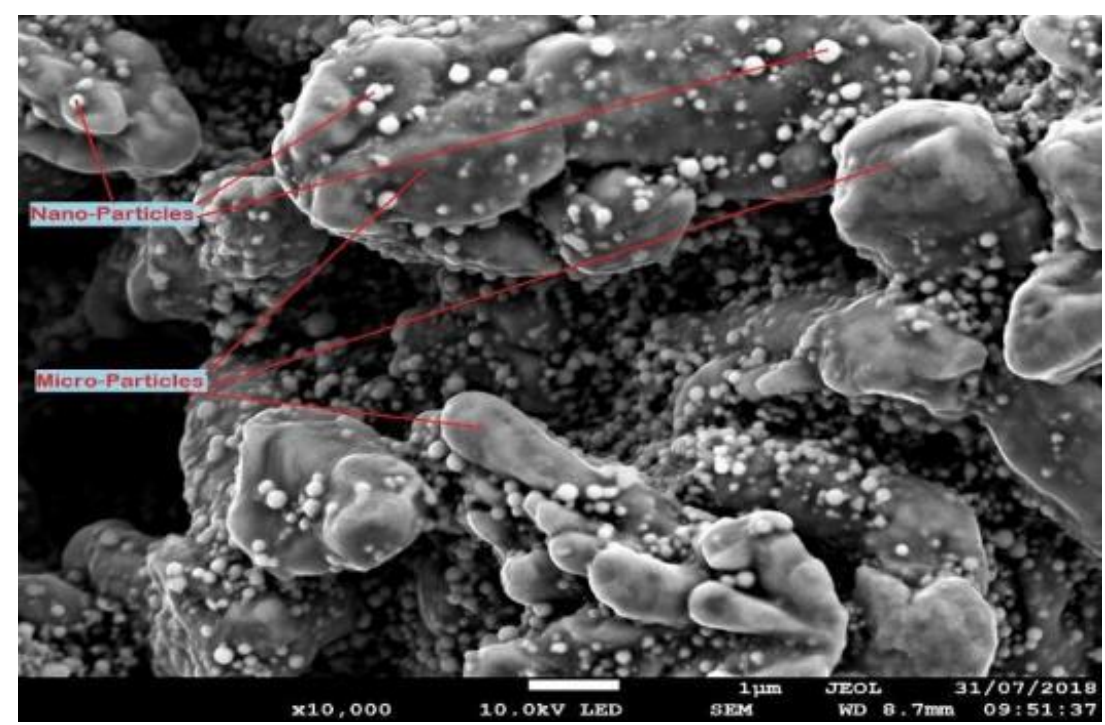

Figure 3. 5 identifying the difference between nano and micro-particles

The un-sintered $100 \mathrm{~N}$ film is made entirely from nano-particles, Figure 3.6 (a), with size distribution from around $20 \mathrm{~nm}$ to $200 \mathrm{~nm}$. Each particle is spherical and distinct from its neighbours, indicating that no sintering has occurred during the drying process. There are voids present in the structure which it is hypothesized are created when particles coalesce as the film contracts during the drying process leading to voids in the packing. Both 50N50M and 20N80M have a similar appearance, illustrate that both materials were a mixture of nano and micro-particles Figure 3.6 (b) and (c). As expected, there is a higher percentage of micro-particles in $20 \mathrm{~N} 80 \mathrm{M}$ ink than 50N50M. In the dried film, the spherical nano-particles lie on the micro particles but are not melded to the micro particles and remain as distinct nano-particles. The micro particles are of the order of $3-5 \mu \mathrm{m}$ size and are irregular in shape. The edges and surface of the micro particles are smooth, and there are significant voids between the particles leading to a lower packing density. 
Chapter 3

(a) $100 \mathrm{~N}$

(b) $50 \mathrm{~N} 50 \mathrm{M}$

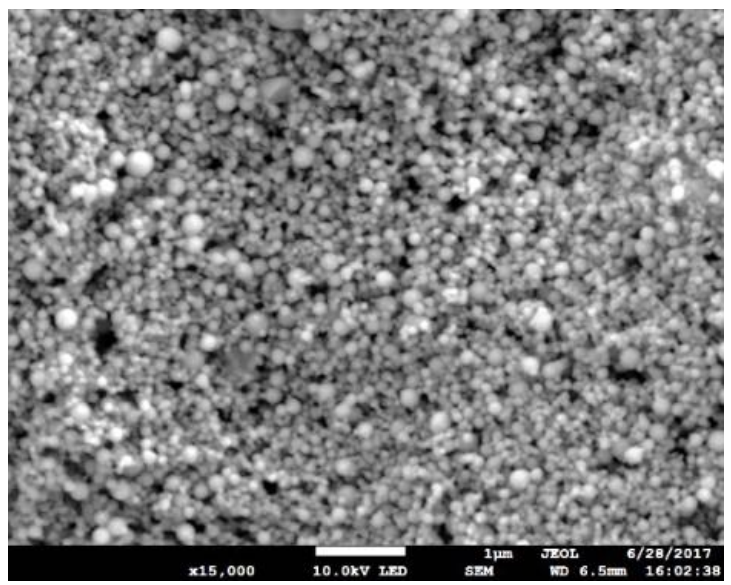

(c) $20 \mathrm{~N} 80 \mathrm{M}$
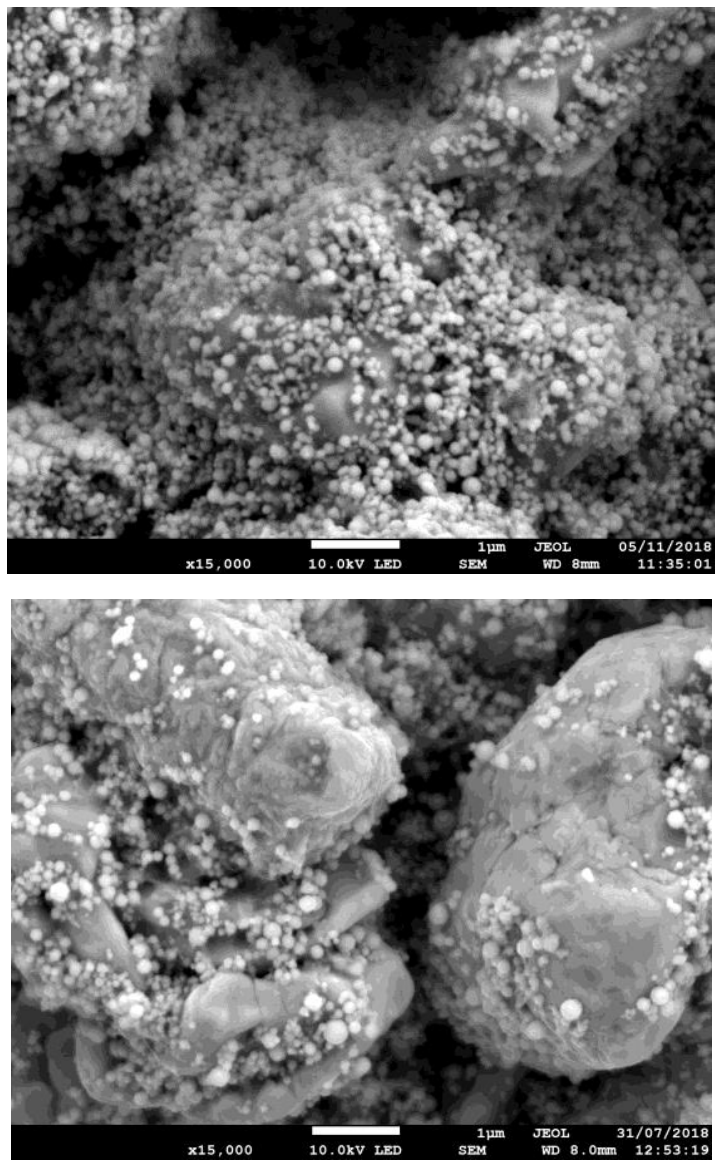

Figure 3. 6 Dry screen-printed inks at $80^{\circ} \mathrm{C}$ for 30 minutes 


\subsection{4: X-ray diffraction measurement (XRD)}

$\mathrm{X}$-ray diffraction was employed to examine the structural and compositional properties of the printed ink materials, depending on the crystal growth of the copper grain structures [82], [135], [136]. Examination of the printed copper film shows that diffraction peaks with intensities appear at angles corresponding $2 \Theta 111,200$ and 220, this means the copper is present with copper (I) oxide, Figure 3.7.

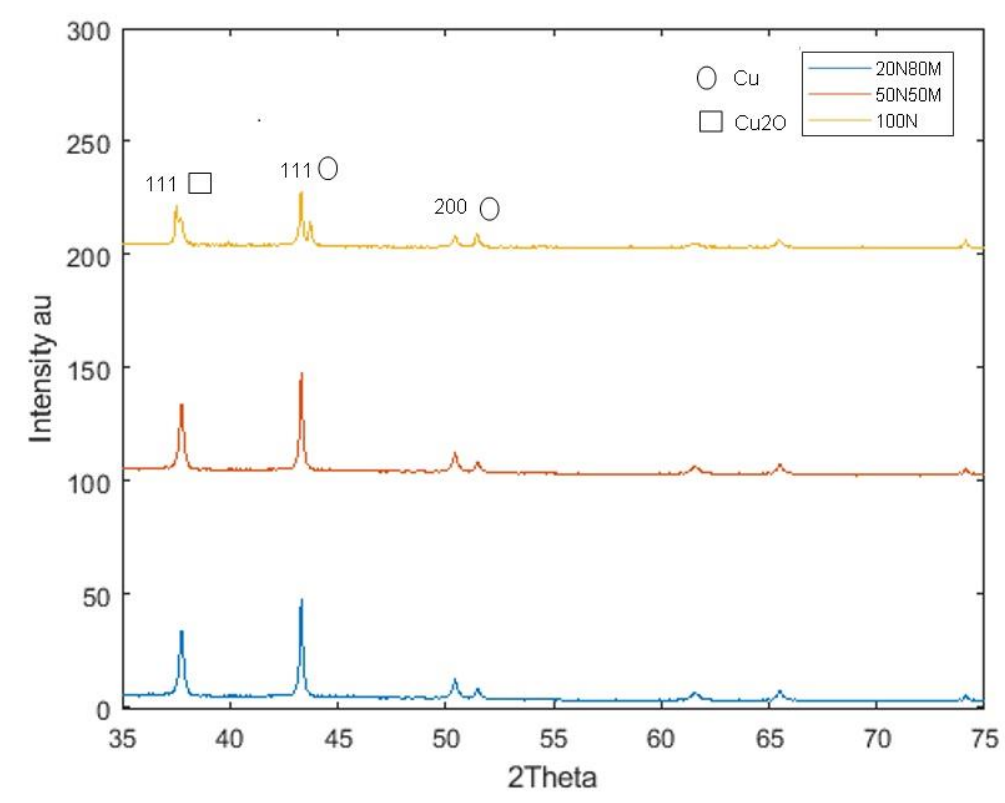

Figure 3. 7 XRD samples dried for 30 minutes at $80^{\circ} \mathrm{C}$

The first peak 111 illustrated the $\mathrm{Cu}_{2} \mathrm{O}$ suggests that the oxidation of copper particles when inks have been dried in air. The second peak of 200 is the copper material. The $\mathrm{Cu}_{2} \mathrm{O}$ layer microstructure of the material dried under ambient condition was carried out to confirm the potential case of copper oxidation, which were confirmed with all materials $(100 \mathrm{~N}, 50 \mathrm{~N} 50 \mathrm{M}$ and 20N80M)

\section{3: Thermal sintering under the ambient condition}

As a control, thermal sintering was applied to thermally sinter the printed copper without any inert or reducing atmosphere. This application will allow an understanding of the role of any oxygen present in the atmosphere. All samples were thermally sintered for 60 mins at $200^{\circ} \mathrm{C}$ in the oven without gas control. Three inks sintered with a significant change in surface colour, Figure 3.8. The dark grey colouring of the $100 \mathrm{~N}$ ink would suggest that there is considerable oxidation of the 
sample with the formation of copper oxide. There is a less radical change in observable colour for the $20 \mathrm{~N} 80 \mathrm{M}$ and $50 \mathrm{~N} 50 \mathrm{M}$ as more micro-content in this ink, which is likely to be related to the lower highly reactive nano-particle content.



Figure 3. 8 Thermally Sintered samples for 60 mins at $200{ }^{\circ} \mathrm{C}$ in the oven

\subsection{1: Resistance measurement (film conductivity)}

No conductivity could be measured from the sintered samples, and this high resistance confirmed the possibility of quickly oxidation materials caused by the absence of a reducing atmosphere [137], [138], [56]. The temperature applied plays the primary role in the oxidation reaction as higher temperature introduce higher energy which leads to a faster oxidation reaction [35].

\subsubsection{SEM sintering under ambient condition}

The thermally sintered samples were investigated using the SEM with applied magnification $(15000 \mathrm{X})$. A severe structure difference resulted with all materials, which also explains the low conductivity obtained. Both 100N and 50N50M have similar SEM images which illustrated that both materials contain a high ratio of nanoparticles Figure 3.9 (a,b). On the contrary of ink 20N80M which show high oxidization as content more micro particles, Figure 3.9, c and Figure 3.12. 
Chapter 3

(a) $100 \mathrm{~N}$

(b) $50 \mathrm{~N} 50 \mathrm{M}$

(c) $20 \mathrm{~N} 80 \mathrm{M}$
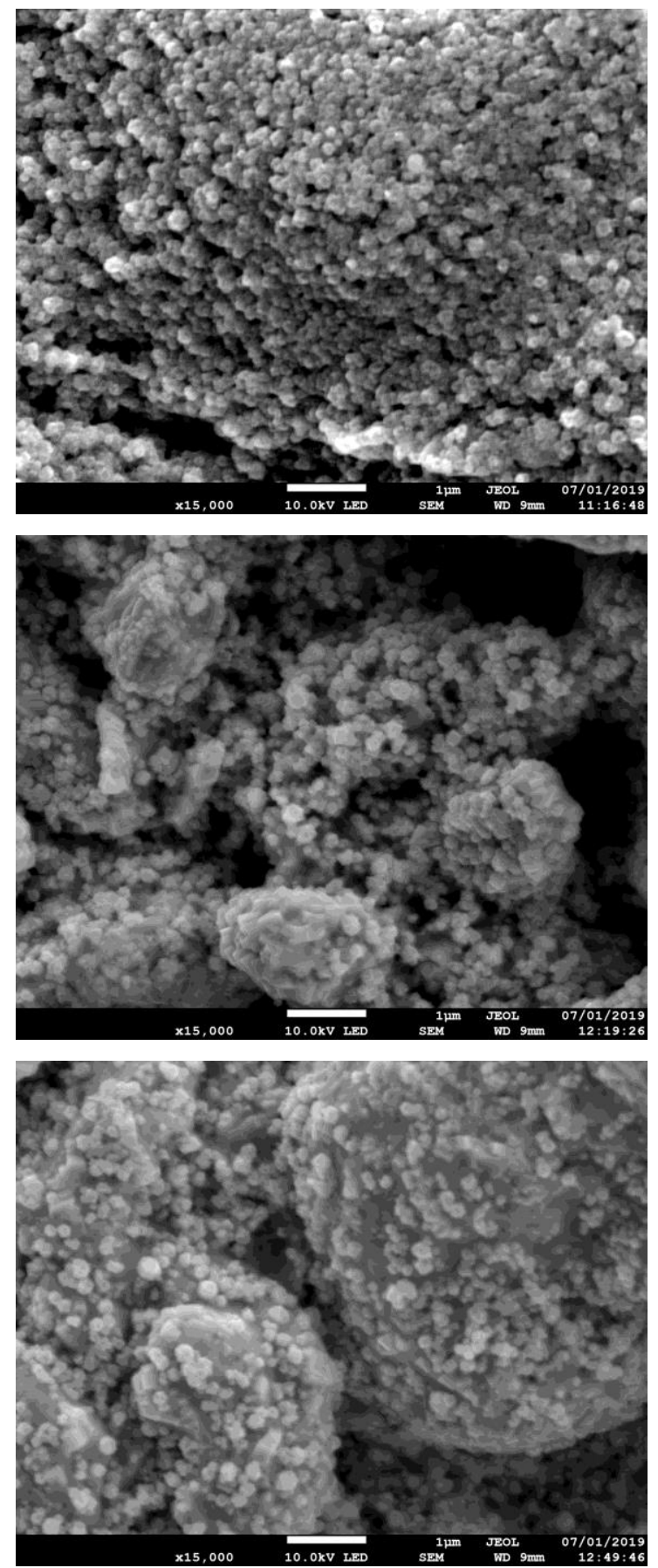

Figure 3. 9 SEM of films sintered in air at $200^{\circ} \mathrm{C}$ for 30 minutes 


\subsection{3: (EDS) examination sintering under the ambient condition}

The EDS of the examined printed materials displayed 100N ink in Figure 3.10, 50N50M ink in Figure 3.11 and 20N80M ink in Figure 3.12.
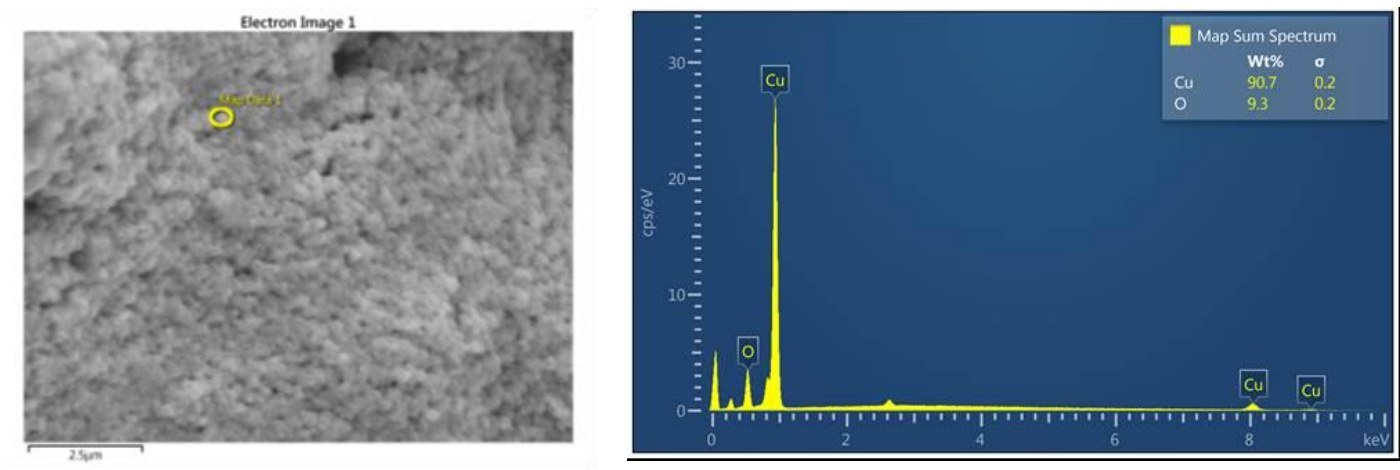

Figure 3. 10 EDS: 100N ink sintered under ambient condition
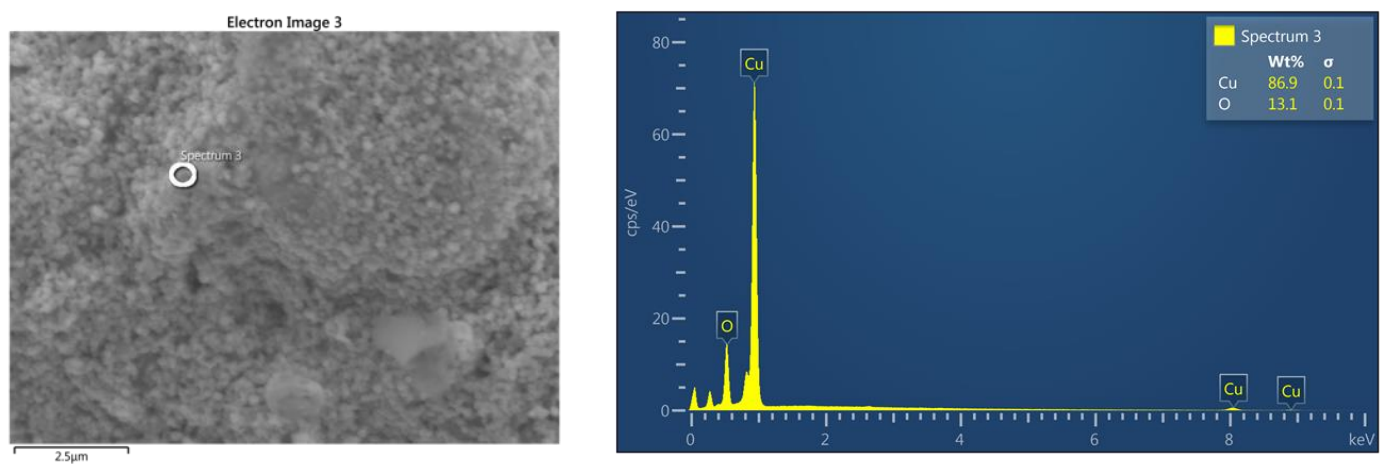

Figure 3. 11 EDS: 50N50M ink sintered under ambient condition
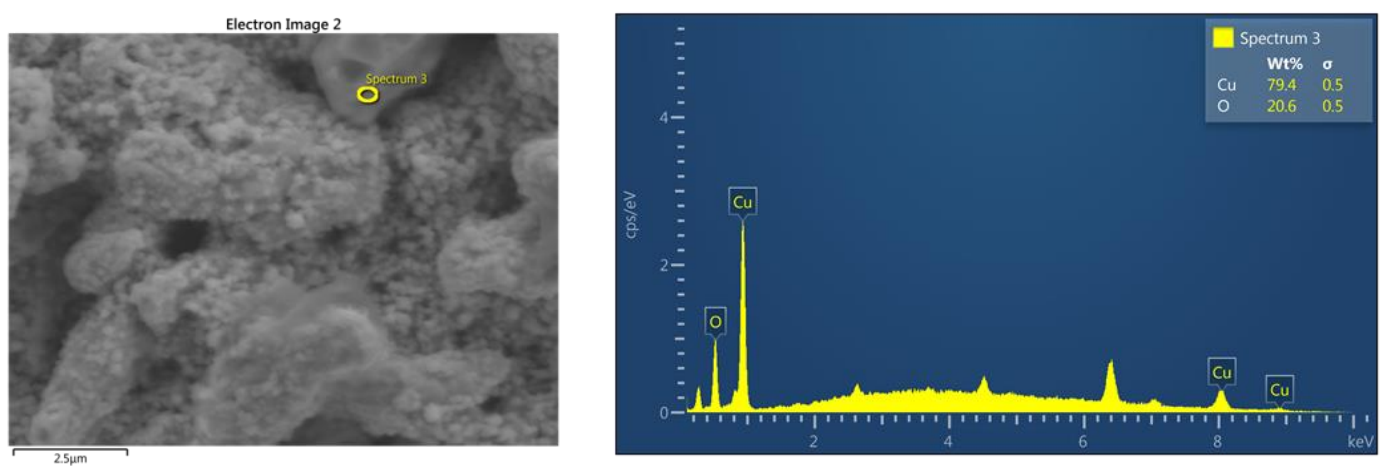

Figure 3. 12 EDS: 20N80M ink sintered under ambient condition

All materials show an oxidation behaviour when sintered under the absence of a reducing atmosphere. The highest oxidation percentage resulted with ink 20N80M which was (20.6 oxide to 79.4 copper) attributed to the high percentage of micro particles in this ink; this also can be seen and supported by the SEM images. Larger particles introduce higher porosity which leads to higher oxidation potential [139]. 
Although an initial oxide shell covers each particle, reaction or oxidation then is controlled by diffusion of oxygen and copper towards each other through the growing oxide shell. The larger oxide shell provided with the micro-particles ink leads to larger oxidisation area in the absence of reducing atmosphere which makes the oxidation possibility higher than nano-particles which introduce a smaller oxide shell area [139].

\subsection{4 (XRD) examination sintering under the ambient condition}

X-ray diffraction for the examined all printed materials represented in Figure 3.13 shows that diffraction peaks with a weak intensity appear at angles corresponding $2 \Theta$ 111,200 and 220 for $100 \mathrm{~N}$ ink, this means the copper (I) oxide resulted [140]. The XRD results of the nano-micro inks also displayed in Figure 3.13 showed a slight peak at all angles, and this means these materials were severely affected by the absence of reducing atmosphere. Overall, the XRD profile when the sample is sintered without the reducing atmosphere presence show an increase in the relative $\mathrm{Cu}_{2} \mathrm{O}$ peak, and it was nearly at the strength of the $\mathrm{Cu}$ peak. The weak growth of the particles has a nondiscriminatory growth and agglomerated along the (111) and (200) direction with nano-micro material. Therefore, the peak and the quantity are related to structure factor and volume. Explanation in simple terms of the XRD results as intensity peaks were more substantial with $100 \mathrm{~N}$ ink.

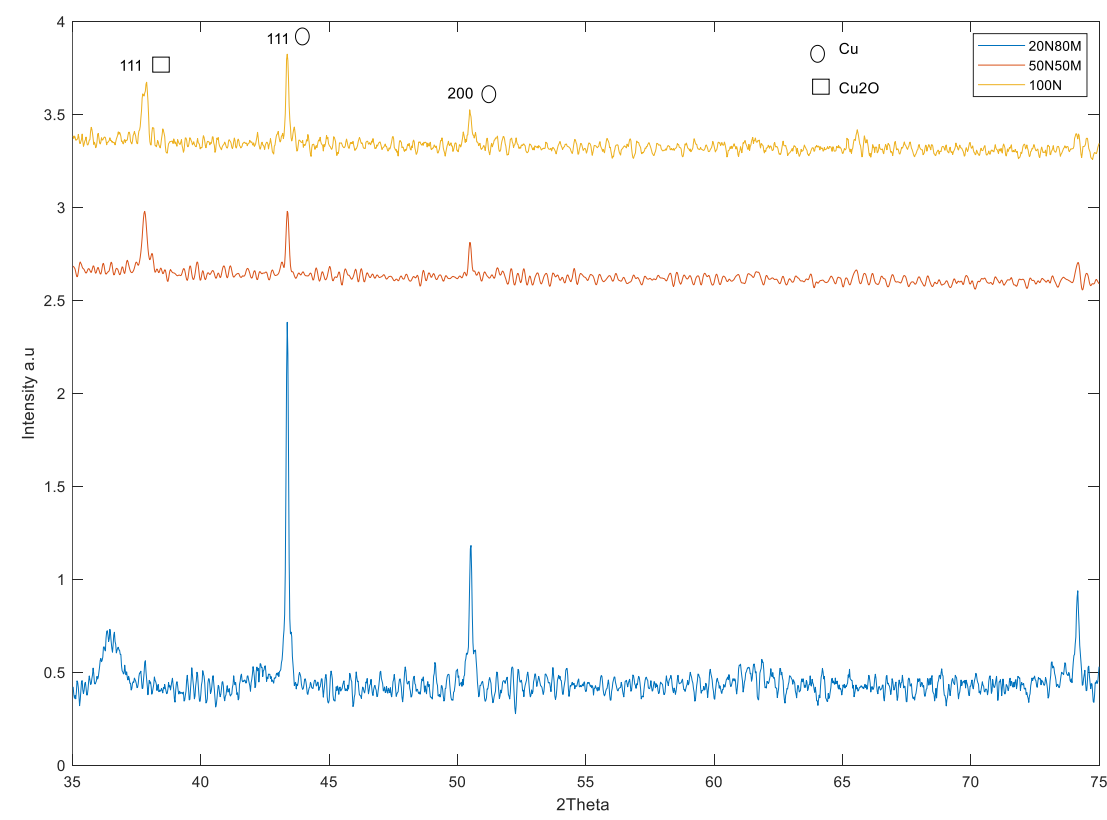

Figure 3. 13 XRD samples dried for 60 minutes at $200^{\circ} \mathrm{C}$ 


\subsubsection{Summary}

The results have clearly illustrated that the sintering of copper inks under ambient gas conditions leads to significant oxidation, even at the lowest temperature. Thus, a reducing or inert atmosphere must be utilized.

\subsection{Thermal sintering under controlled oven atmosphere}

The main challenge with using copper nano-particles is the oxidation during sintering, as nano or micro-copper is readily oxidized at temperatures over $130{ }^{\circ} \mathrm{C}$, which leads to a poor conductivity [141], [142], [143]. Consequently, the sintering under inert / reducing atmospheres employed in this study using the oven with a nitrogen condition, formic acid condition and of mixed 5\% hydrogen and 95\% nitrogen was investigated.

The effect of applying individually nitrogen, formic acid and $\mathrm{H}_{2} / \mathrm{N}_{2}$ atmosphere during the sintering process on the copper films was examined over a temperature range (175$275^{\circ} \mathrm{C}$ ) and periods between 30 and 120 minutes in 30 -minute intervals. This represents a sizeable full factorial of experimental conditions and represents a significant experimental undertaking. As the results will indicate, this methodology was vindicated due to the complex interactions between the material and the sintering conditions with the optimum sintering for each material being a function of time, temperature, and atmosphere.

\subsection{1: Resistance measurement (film conductivity) under controlled atmosphere}

The thermal response of each material is significantly different and is also a function of the sintering environment. In comparing the line resistance of a nominally $600 \mu \mathrm{m}$ width line, the $100 \mathrm{~N}$ nano-particle ink continuously produces the lowest line resistance, Figure 3.15 (a-c), and is relatively insensitive to the sintering temperature in the $175{ }^{\circ} \mathrm{C}-225^{\circ} \mathrm{C}$ range and extended exposure time. Above $225^{\circ} \mathrm{C}$, extending the sintering time has a detrimental effect on the conductivity of the material with incremental increases in resistance with longer sintering time. Both the inks containing micro particles exhibit higher initial resistances than the nano-particle ink, Figure 3.15 (d-f) \& Figure 3.15 (g-i). In contrast to the $100 \mathrm{~N}$ ink, increasing sintering temperature reduces the resistance in each case. There is a more complex interaction between the sintering time and the resistance for micro-nano blend inks. 
It is possible to sinter all three inks in a nitrogen environment. Still, the resistance of the printed line is reduced for those produced in a reducing atmosphere is more consistent and controlled. Above $250{ }^{\circ} \mathrm{C}$ and 90 minutes, there was significant decolouration of the substrate as the high-temperature acidic vapour (which had not thermally decomposed to $\mathrm{CO}$ and $\mathrm{H}_{2} \mathrm{O}$ ) reacted with the FTO coating (causing yellowing of the substrate), which resulted in delamination of the printed film. With the reducing atmosphere produced by the $5 \% \mathrm{H}_{2} / \mathrm{N}_{2}$, the absolute values of the conductivity are comparable with that produced by the formic acid-reducing atmosphere. However, the high-temperature sintering does not have such a detrimental effect on the FTO substrate.

There is a substantial increase in resistance with $100 \mathrm{~N}$ at temperatures above $225^{\circ} \mathrm{C}$. Possible reasons for this include oxidation from organic compounds in the film as the binder is burned off, Figure 3.14. Possible used binders in these materials are acrylic and epoxy, which contain oxygen in their structure. Stress cracking between the nanoparticle film as the metallic film expands at a differential rate to the underlying glass substrate. Of the two hypotheses, oxidation is thought to be the most likely scenario since the effect is less pronounced under reducing atmosphere. Under inert conditions, there is nothing to counteract the localized oxidative atmosphere caused by the binder burn off or the other additives such as capping agents [144]. The solvent evaporation can cause another localized oxidative. The possible solvents used in these inks are Trepineol, methoxy-2-propanol and Diproylene Glycol.

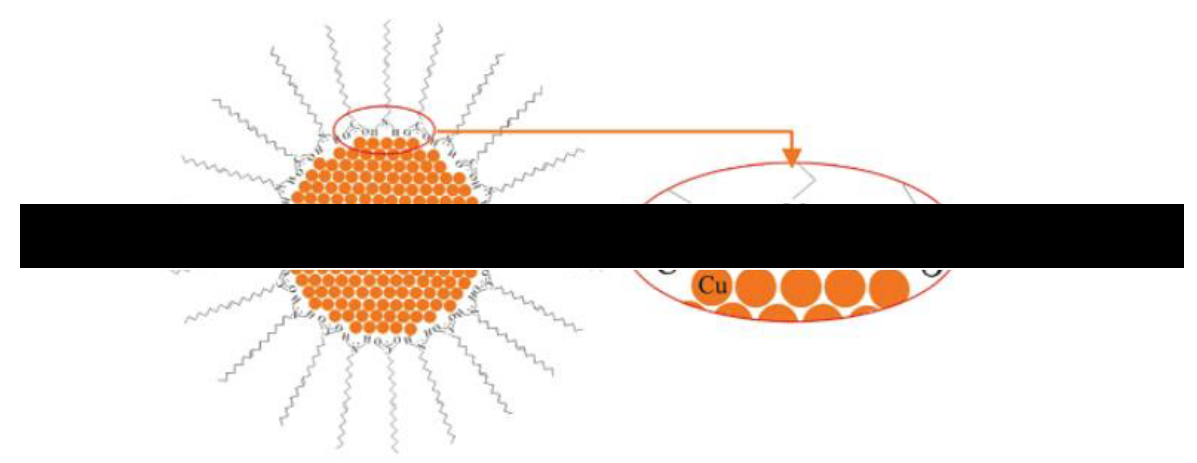

Figure 3. 14 Copper Nanoparticles in the Presence of Capping Agents in Organic Solvent [144] 


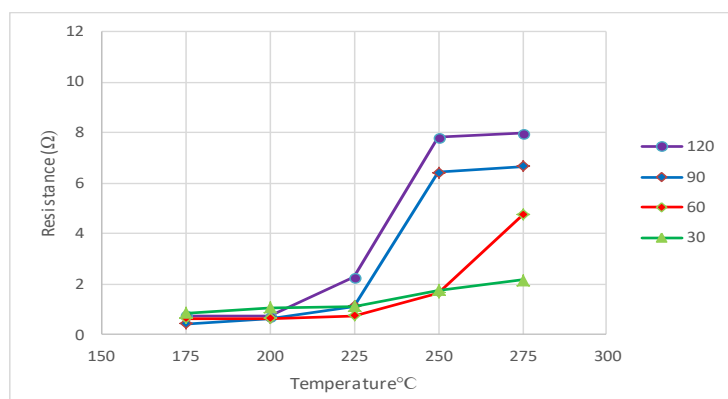

(a) $100 \mathrm{~N}-\mathrm{N}_{2}$

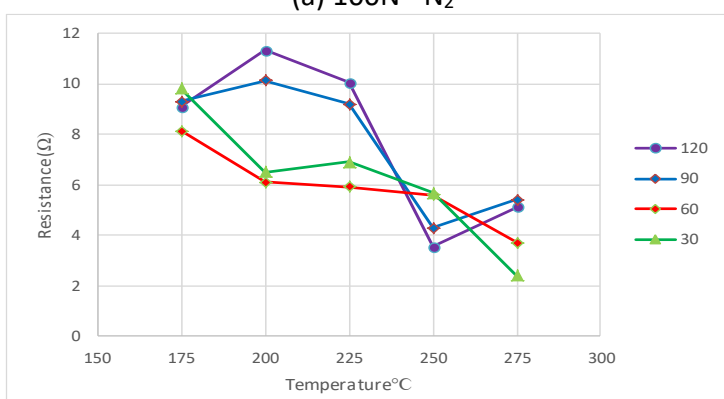

(d) $50 \mathrm{~N} 50 \mathrm{M}-\mathrm{N}_{2}$

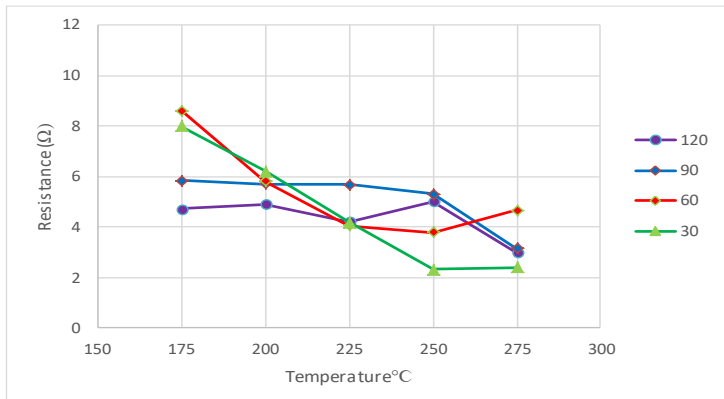

(g) 20N80M - $\mathrm{N}_{2}$

(h) 20N80M - Formic acid
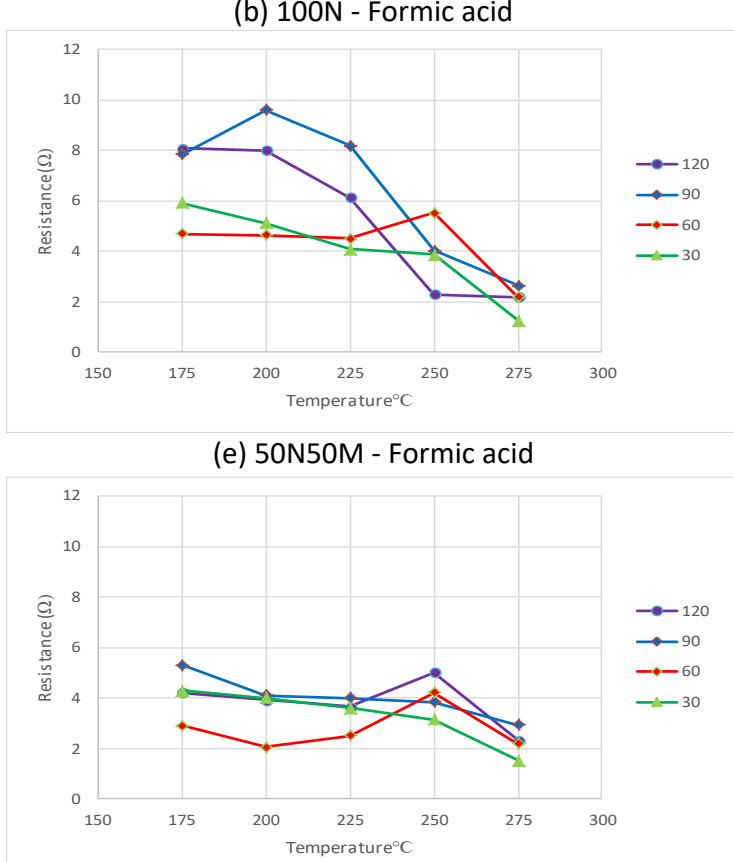

(e) 50N50M - Formic acid

minutes.

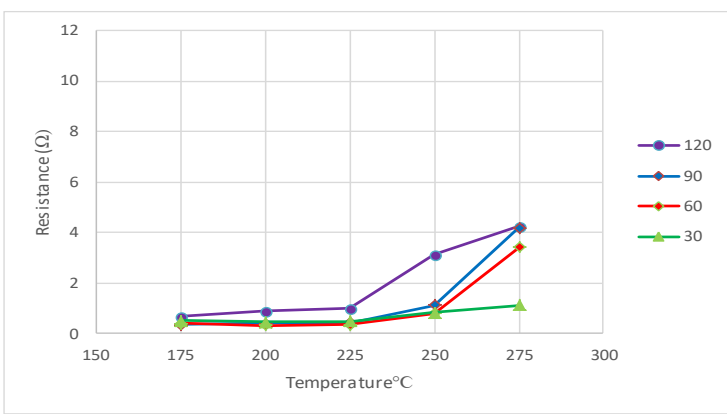

(c) $100 \mathrm{~N}-5 \% \mathrm{H}_{2} / \mathrm{N}_{2}$

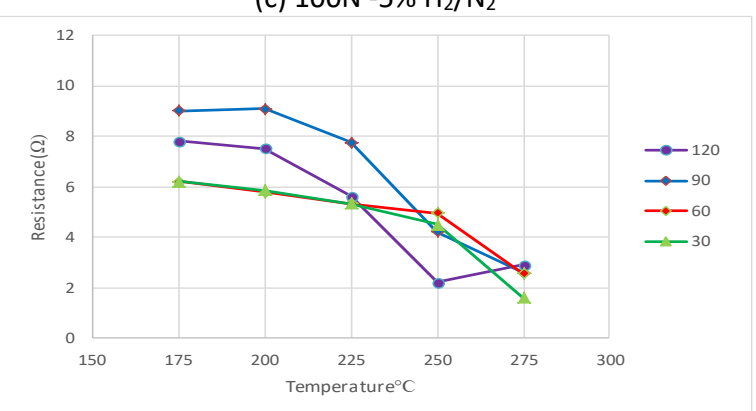

(f) $50 \mathrm{~N} 50 \mathrm{M}-5 \% \mathrm{H}_{2} / \mathrm{N}_{2}$

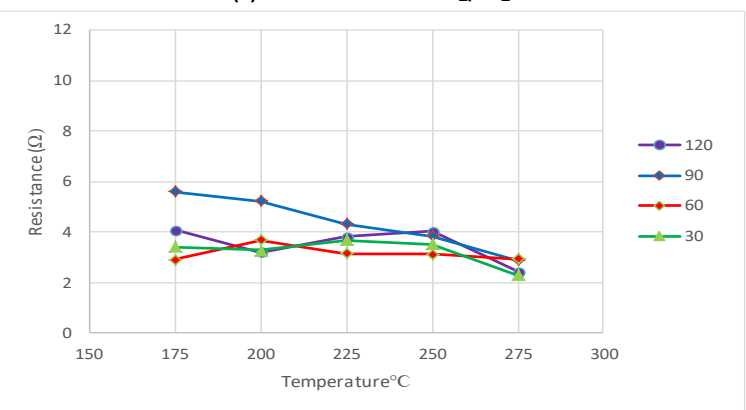

(i) $20 \mathrm{~N} 80 \mathrm{M}-5 \% \mathrm{H}_{2} / \mathrm{N}_{2}$

Figure 3. 15 Line resistance for a nominal $600 \mu \mathrm{m}$ wide line of $30 \mathrm{~mm}$ length under each sintering environment at residence times of $30,60,90$ and 120 


\subsection{2: Sheet resistance by 4-point probe under controlled atmosphere}

Similar behaviour to that observed with the line resistance is found with the film sheet resistance, Figure 3.16 (a-i). However, some differences are observed which have been attributed to the geometrical nature of the feature being printed. For the $100 \mathrm{~N}$ ink, below $225^{\circ} \mathrm{C}$, the sheet resistance is mostly independent of the sintering time but extended times at higher temperatures result in significant increases in resistance, Figure 3.16 (a-c). As with the line resistance, the negative impact of the higher temperatures is independent of the sintering atmosphere. Similar behaviour to the resistance of the printed line was observed at below $225^{\circ} \mathrm{C}$ for the sheet resistance of the 50N50M and 20N80M nano / micro inks. Above $250{ }^{\circ} \mathrm{C}$, there is an increase in the sheet resistance at all sintering times for the 50N50M ink and the sensitivity to temperature and residence time reduces progressively with the quality of the reducing atmosphere. For the 20N80M ink, there is a more complex interaction between the sintering time and temperature, Figure 3.16 (g-i). At low sintering times, it is advantageous to sinter at a higher temperature, while an extended sintering time is detrimental at the higher temperature.

The lowest sheet resistance achieved is $(0.904 \mathrm{~m} \Omega / \square)$ at $225^{\circ} \mathrm{C}$ sintered for 30 minutes, which is also limited deference compared to the sheet resistance achieved with $250^{\circ} \mathrm{C}$ for 30 minutes $(0.951 \mathrm{~m} \Omega / \square)$. These results are akin to the sheet resistance obtained under nitrogen condition at the same temperature. The highest was obtained with $275^{\circ} \mathrm{C}$ for 120 minutes $(4.1 \mathrm{~m} \Omega / \square)$. For $50 \mathrm{~N} 50 \mathrm{M}$ ink -sheet resistance is higher than the sheet resistance resulted from 20N80M material. A significant enhancement on sheet resistance presented by formic acid atmosphere compares to the nitrogen condition. For the $20 \mathrm{~N} 80 \mathrm{M}$ ink, there is a more complex interaction between the sintering time and temperature, Fig. 3.16-g. At low sintering times, it is advantageous to sinter at a higher temperature, while an extended sintering time is detrimental at the higher temperature. The lowest sheet resistance achieved with $20 \mathrm{~N} 80 \mathrm{M}$ ink is $(1.65$ $\mathrm{m} \Omega / \square)$ at $275^{\circ} \mathrm{C}$ sintered for 30 minutes, which is limited deference compared to the sheet resistance achieved with $225^{\circ} \mathrm{C}$ for 120 minutes $(3.67 \mathrm{~m} \Omega / \square)$. The highest obtained under the formic condition at $175^{\circ} \mathrm{C}$ for 30 minutes $(21.30 \mathrm{~m} \Omega / \square)$. 


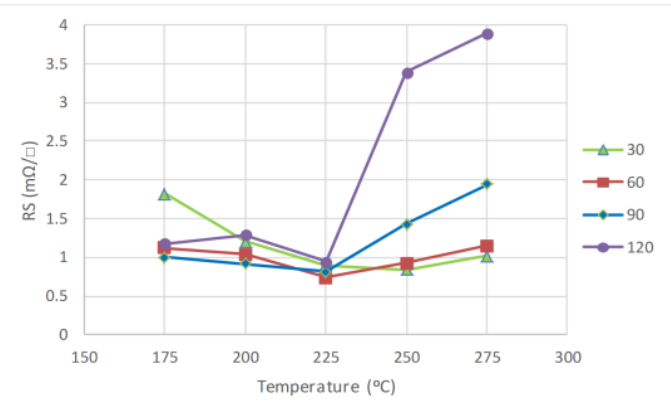

(a) $100 \mathrm{~N}-\mathrm{N}_{2}$

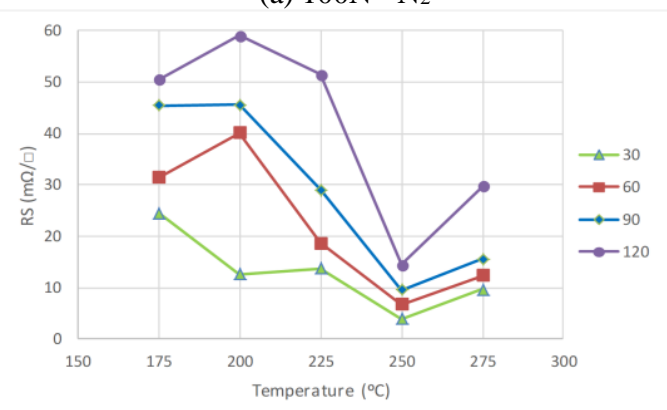

(d) $50 \mathrm{~N} 50 \mathrm{M}-\mathrm{N}_{2}$

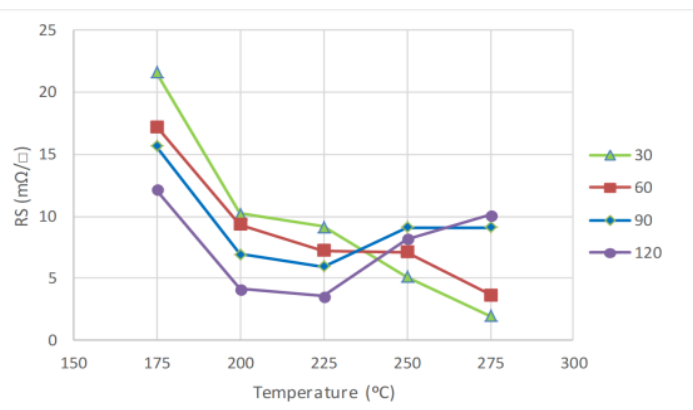

(g) 20N80M - $\mathrm{N}_{2}$

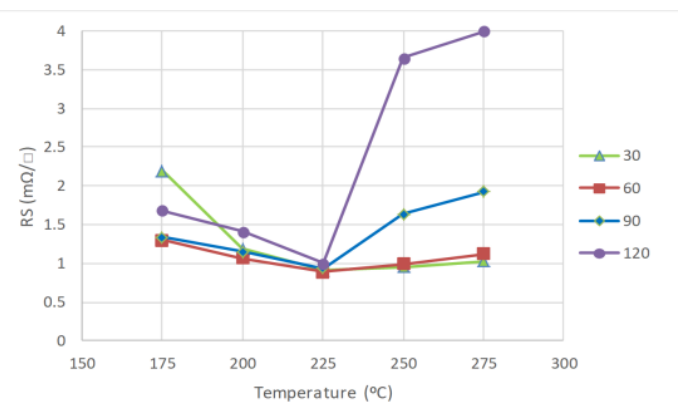

(b) $100 \mathrm{~N}$ - Formic acid

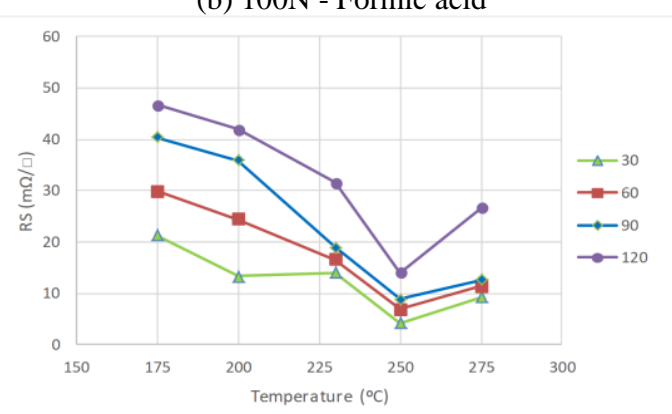

(e) 50N50M - Formic acid

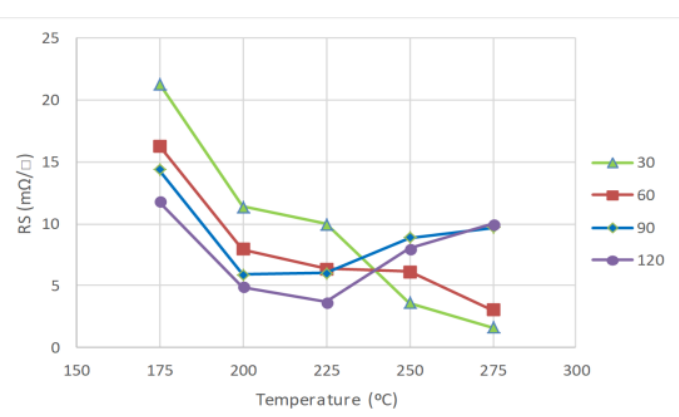

(h) $20 \mathrm{~N} 80 \mathrm{M}$ - Formic acid

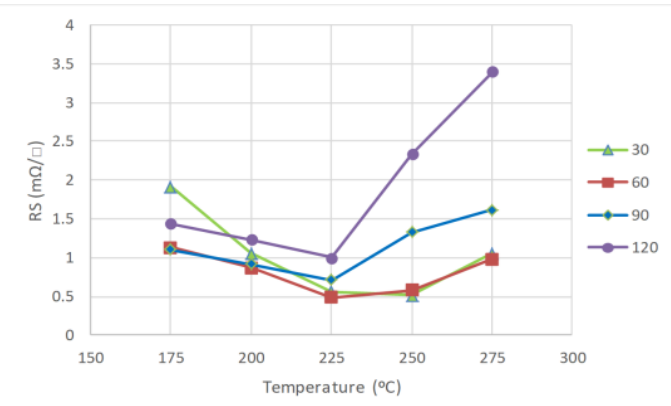

(c) $100 \mathrm{~N}-5 \% \mathrm{H}_{2} / \mathrm{N}_{2}$

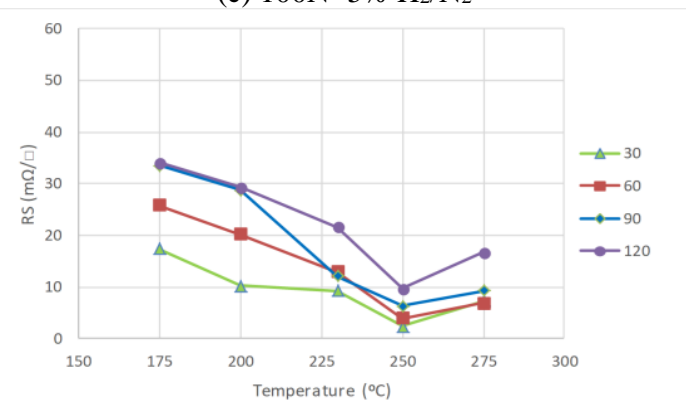

(f) $50 \mathrm{~N} 50 \mathrm{M}-5 \% \mathrm{H}_{2} / \mathrm{N}_{2}$

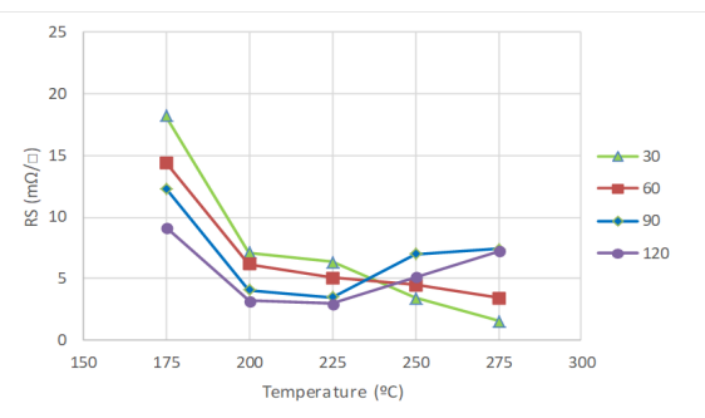

(i) $20 \mathrm{~N} 80 \mathrm{M}-5 \% \mathrm{H}_{2} / \mathrm{N}_{2}$

Figure 3. 16 Sheet resistance of each ink under the $\mathrm{N}_{2}$, Formic acid and $5 \% \mathrm{H}_{2} / \mathrm{N}_{2}$ sintering environements at residence times of 30,60 , 90 and 120 minutes. 


\subsection{3: TLM measurement of sintering under controlled atmosphere}

TLM measurement carried out measuring the contact resistance after the sintering process under reducing condition, the contact resistance is strongly affected by the sintering time in a nitrogen environment, but less so with lower temperature applied.

The trends observed in sheet resistance are generally repeated with the contact resistance, Figure 3.17 with typical contact resistances of between $0.5 \Omega$ and $12 \Omega$. The 120-minute sintering time has been omitted from the Figure as FTO / printed film damage was often observed, leading to delamination causing significant variation in the data. Similarly, the $100 \mathrm{~N}$ at $275^{\circ} \mathrm{C}$ have been omitted as failure film adhesion failure occurred. For the $100 \mathrm{~N}$ material, minimum contact resistances are mostly unaffected by sintering temperatures above $200^{\circ} \mathrm{C}$. Still, they can suffer a detrimental effect if held at the highest temperature for a prolonged period, Figure 3.17 (a-c). Contact resistances for the micro/nano-particle inks are around 2 to 10 times that observed with the pure nano inks, Figure 3.17 (d-i). This difference is likely to be due to the reduced contact area interface associated with larger particles. For the 50N50M ink, there is a general reduction in the contact resistance as the temperature is increased under all sintering conditions with a lower sintering time also consistently producing the minimum contact resistance. For the $20 \mathrm{~N} 80 \mathrm{M}$ ink, the contact resistance is strongly affected by the sintering time in a nitrogen environment, but less so under other reducing conditions. Under $5 \% \mathrm{H}_{2} / \mathrm{N}_{2}$ reducing conditions, both the micro/nano blended materials exhibit the highest contact resistance.

Although, at high temperature, the film begins to tarnish and this tarnishing lead to almost artificially high resistance, which then leads to an increase in the gradient of the line. The straight-line fit through only five points, it becomes more sensitive to one point which out or far from the others as the difference between point 1-5 is around 125. As each point weighted, a negative intercept obtained shown in Figure 3.18, which resulted $(-12 \Omega)$ contact resistance. 


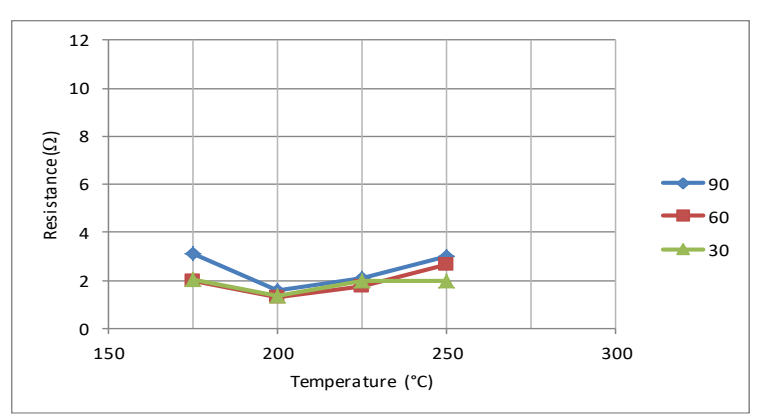

(a) $100 \mathrm{~N}-\mathrm{N}_{2}$

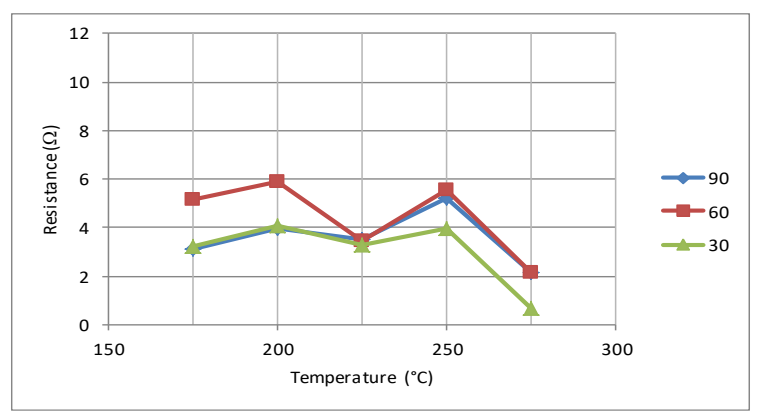

(d) $50 \mathrm{~N} 50 \mathrm{M}-\mathrm{N}_{2}$

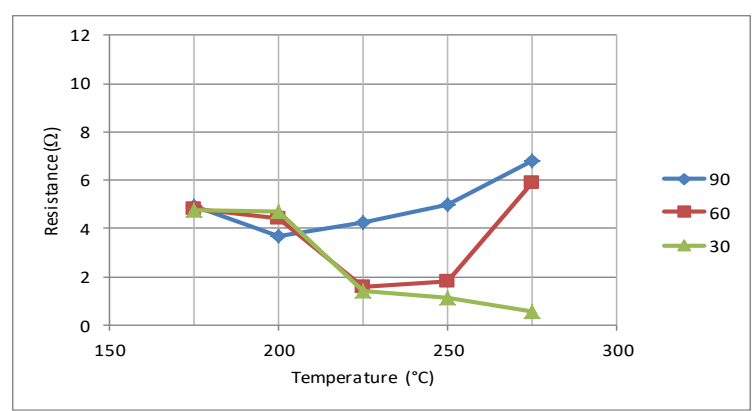

(g) $20 \mathrm{~N} 80 \mathrm{M}-\mathrm{N}_{2}$

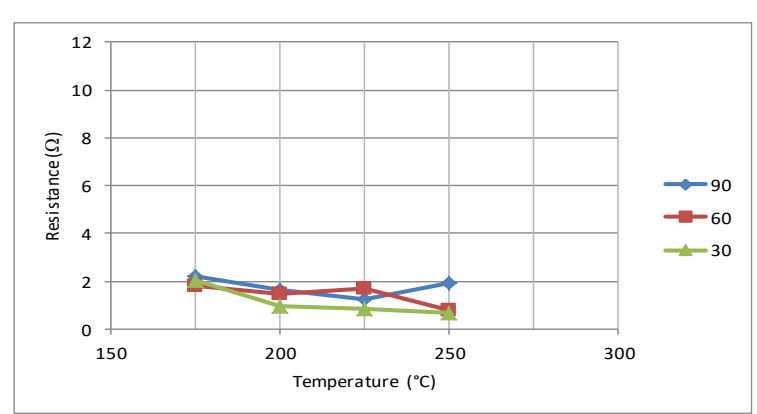

(b) $100 \mathrm{~N}$ - Formic acid

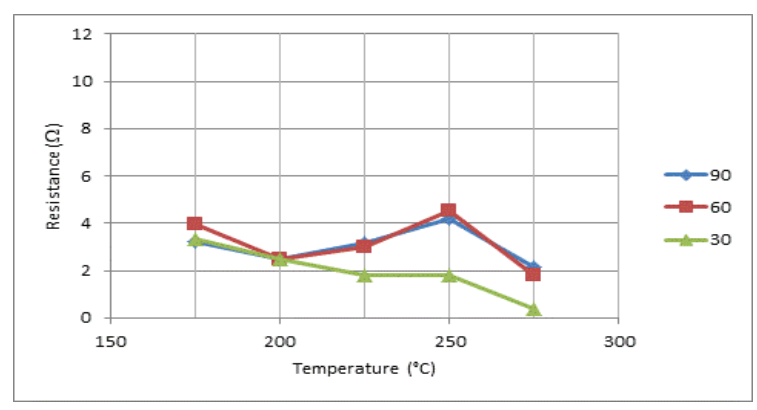

(e) 50N50M - Formic acid

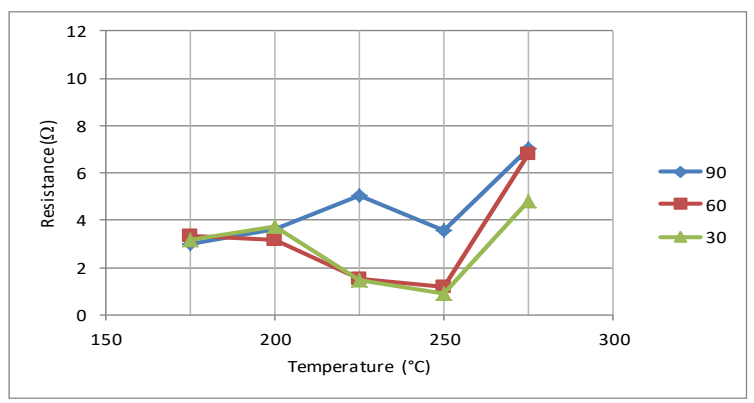

(h) $20 \mathrm{~N} 80 \mathrm{M}$ - Formic acid

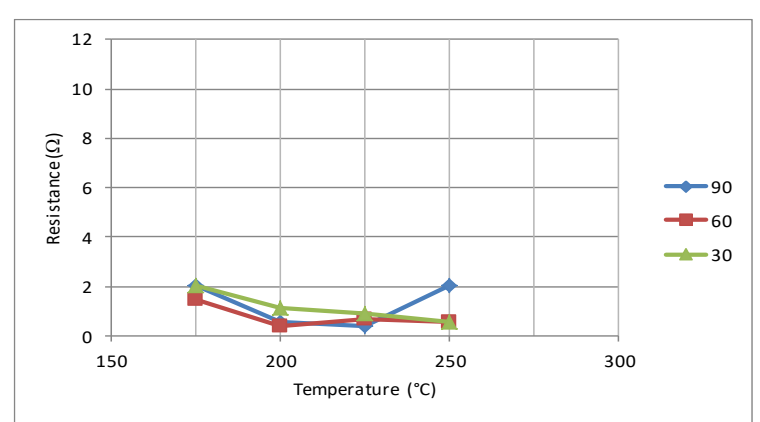

(c) $100 \mathrm{~N}-5 \% \mathrm{H}_{2} / \mathrm{N}_{2}$

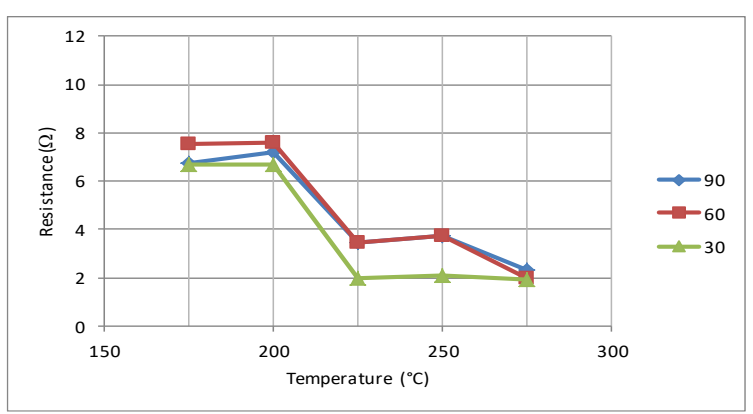

(f) $50 \mathrm{~N} 50 \mathrm{M}-5 \% \mathrm{H}_{2} / \mathrm{N}_{2}$

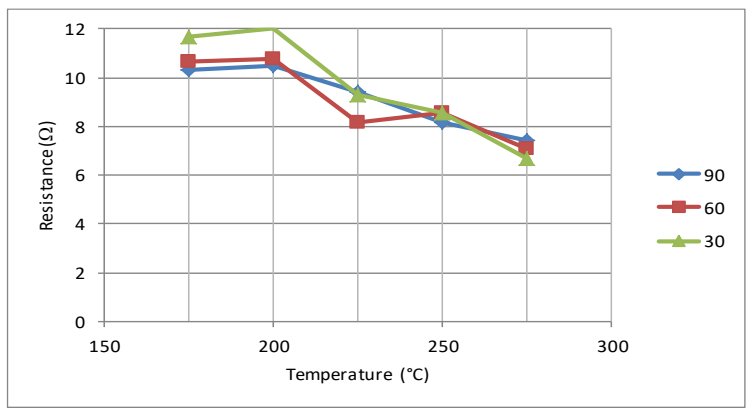

(i) $20 \mathrm{~N} 80 \mathrm{M}-5 \% \mathrm{H}_{2} / \mathrm{N}_{2}$

Figure 3. 17 Contact resistance of each ink under the N2, Formic acid and $5 \% \mathrm{H}_{2} / \mathrm{N}_{2}$ sintering environements at residence times of 30, 60 and 90. 


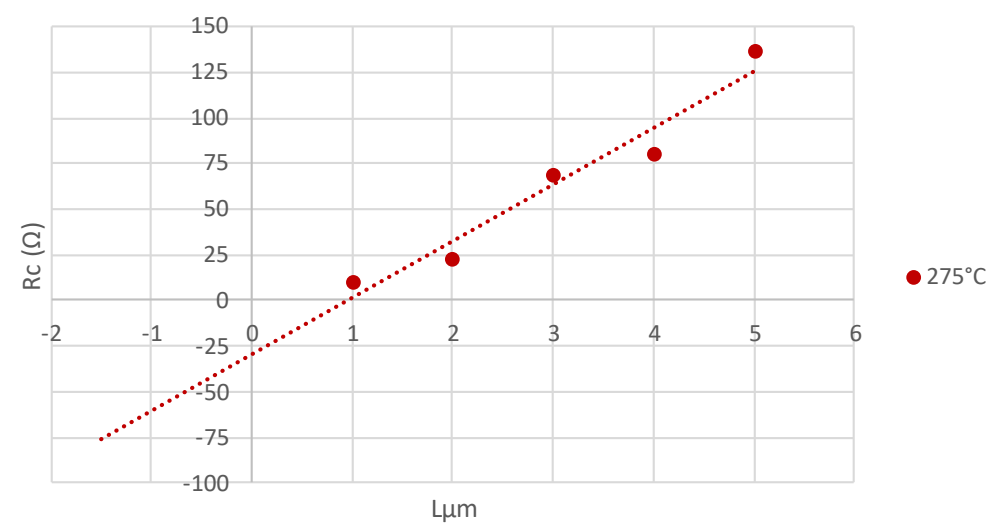

Figure 3. 18 Contact resistance under nitrogen atmosphere at $275^{\circ} \mathrm{C}$ for 30 minutes

\subsection{4: Adhesion test after thermal sintering under controlled atmosphere}

Adhesion test results after sintering under nitrogen condition with respect to the different ratio of nano-micro particles materials are illustrated in Table 3.2.

Table 3. 3 (Adhesion tape test result after thermal sintering)

\begin{tabular}{|c|c|c|c|c|}
\hline Ink (nano-micro) & Temperature ${ }^{\circ} \mathrm{C}$ & $\mathrm{N}_{2}$ & $\begin{array}{c}\text { Formic } \\
\text { Acid }\end{array}$ & $\mathrm{H}_{2} / \mathrm{N}_{2}$ \\
\hline \multirow{3}{*}{$100 \mathrm{~N}$} & 200 & $4 \mathrm{~B}$ & $4 \mathrm{~B}$ & $5 \mathrm{~B}$ \\
\cline { 2 - 5 } & 250 & $2 \mathrm{~B}$ & $0 \mathrm{~B}$ & $2 \mathrm{~B}$ \\
\cline { 2 - 5 } & 275 & $1 \mathrm{~B}$ & $0 \mathrm{~B}$ & $2 \mathrm{~B}$ \\
\hline \multirow{3}{*}{$50 \mathrm{~N} 50 \mathrm{M}$} & 200 & $4 \mathrm{~B}$ & $4 \mathrm{~B}$ & $5 \mathrm{~B}$ \\
\cline { 2 - 5 } & 250 & $4 \mathrm{~B}$ & $3 \mathrm{~B}$ & $5 \mathrm{~B}$ \\
\cline { 2 - 5 } & 275 & $5 \mathrm{~B}$ & $3 \mathrm{~B}$ & $5 \mathrm{~B}$ \\
\hline \multirow{2}{*}{$20 \mathrm{~N} 80 \mathrm{M}$} & 200 & $5 \mathrm{~B}$ & $4 \mathrm{~B}$ & $5 \mathrm{~B}$ \\
\cline { 2 - 5 } & 250 & $5 \mathrm{~B}$ & $4 \mathrm{~B}$ & $5 \mathrm{~B}$ \\
\cline { 2 - 5 } & 275 & $5 \mathrm{~B}$ & $4 \mathrm{~B}$ & $5 \mathrm{~B}$ \\
\hline
\end{tabular}


The $100 \mathrm{~N}$ ink showed a poor adhesion level, particularly at high sintering temperatures. Although the conductivity was improved by employing a nitrogen atmosphere sintering condition, this led to poor adhesion. This effect on adhesion may be because high sintering temperature [145] causes over sintering of printed materials which lead to damage on the bond between the nano-particles ink and the FTO substrate. The evaporation of the binder utilized in the ink preparation with high temperature applied during the sintering process increases the possibility of materials burning. For this reason, high sintering temperature applied on copper nano-micro ink (50N50M and 20N80M) films had higher resistivity and a better level of adhesion strength considering a different path the binder would take to evaporate with this ink [146]. The higher ratio of micro particles the ink contains led to a better adhesion performance, $5 \mathrm{~B}$ at all sintering temperature.

Blending nano-particles with micro particles can be advantageous in that it is likely to be due to the stronger bond associated with larger particles as less effected by the sintering environment. For the 50N50M ink, there is a general increase in the adhesion with all sintering temperature at shorter sintering time. For the $20 \mathrm{~N} 80 \mathrm{M}$ a $5 \mathrm{~B}$ adhesion was obtained at all temperatures because of the higher ratio of micro particles.

\subsection{5: SEM examination sintering under controlled atmosphere}

Thermally sintered material with the presence of reducing atmosphere provided a significant enhancement in resistance. Also improved the uniform morphology throughout the whole printed line, SEM was employed to examine the surface of the sintered film, a considerable change in the colour and surface morphology were observed when reducing atmosphere applied at all temperatures. Figure 3.19 shows the SEM images of the sintered samples under the nitrogen atmosphere, which resulted in the lowest resistance $\left(225{ }^{\circ} \mathrm{C}\right.$ for 60 minutes $)$. A macroscopic examination of the printed lines shows an increase in porosity of the surface film with an increase in the number of surface voids. This increase in film porosity has a detrimental effect on conductivity. Closer inspection shows that the individual nano-particles have necked and melded into each other at their interface, creating a globular network of conductive particles. 

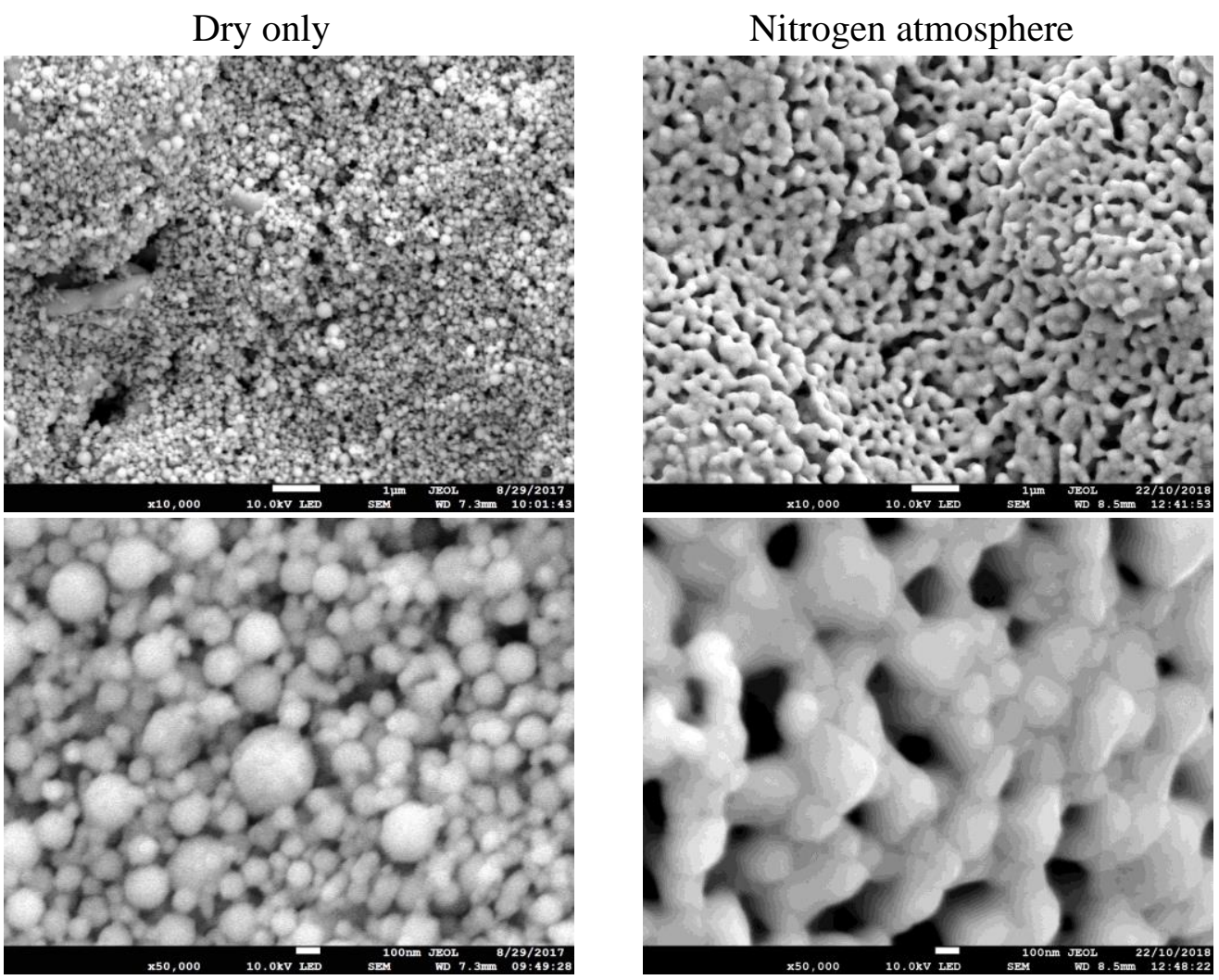

Figure 3. 19 100N SEM of the dried and nitrogen sintered films

The best surface morphology of the micro/nano blends (50N50M and 20N80M) achieved when sintered under nitrogen atmosphere at $275^{\circ} \mathrm{C}$ for 30 minutes, which produced the lowest film resistance sees less significant change due to the sintering process in nitrogen, Figure 3.20. Macroscopically at x 1000, then dried and sintered surfaces are similar in texture, although there is some evidence of enhanced smoothness after sintering, Figure 3.20 (a) and (c). At a higher magnification, there is only partial evidence of melting of the nano-particles on the surface of the micro particles. It is postulated that the necking initially occurs on the nano-particle. Still, the action of contact merging with the microparticle increases the effective particle size and therefore, further melting becomes thermodynamically unfavourable. 
Dry

(a)

50N50M

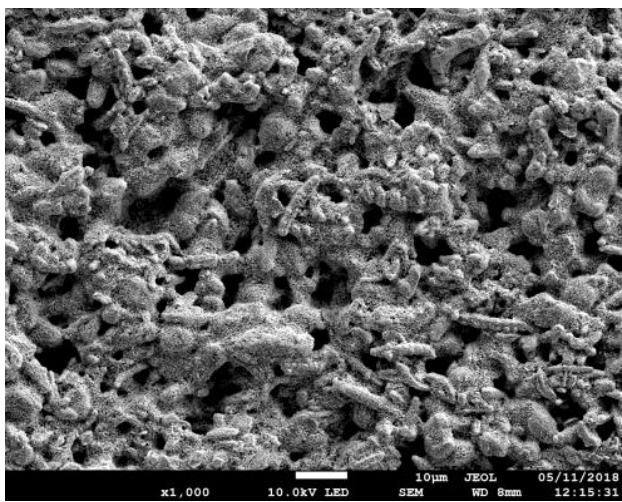

(b)

50N50M

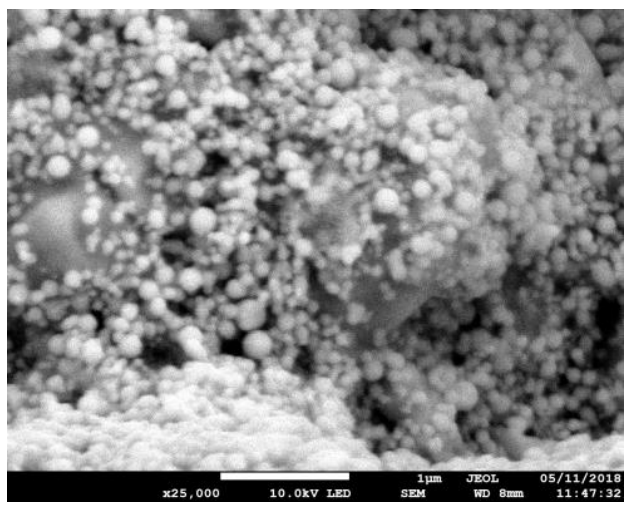

(c)

20N80M
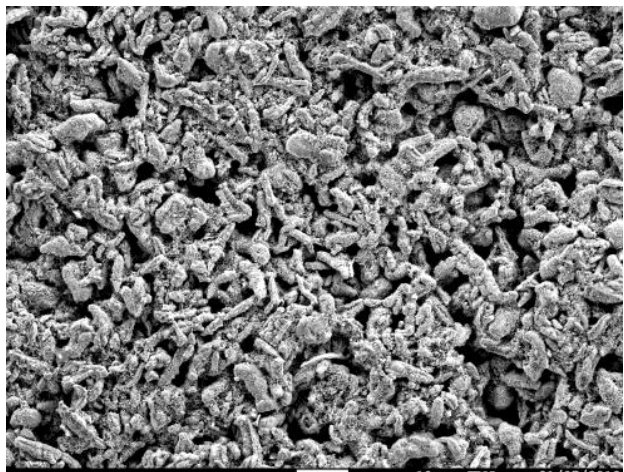

(d)

20N80M

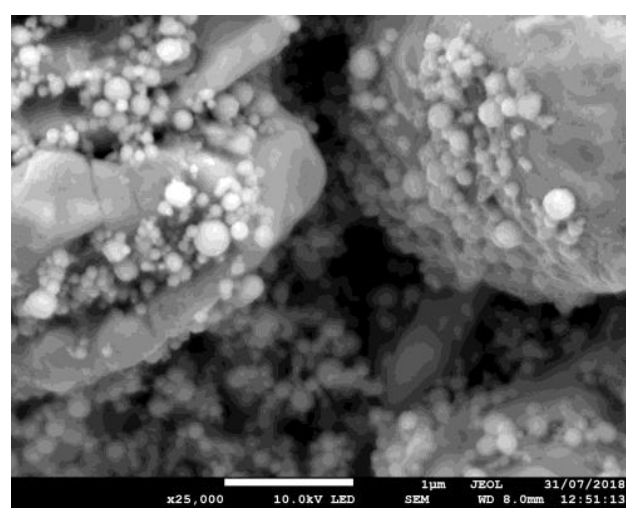

Nitrogen
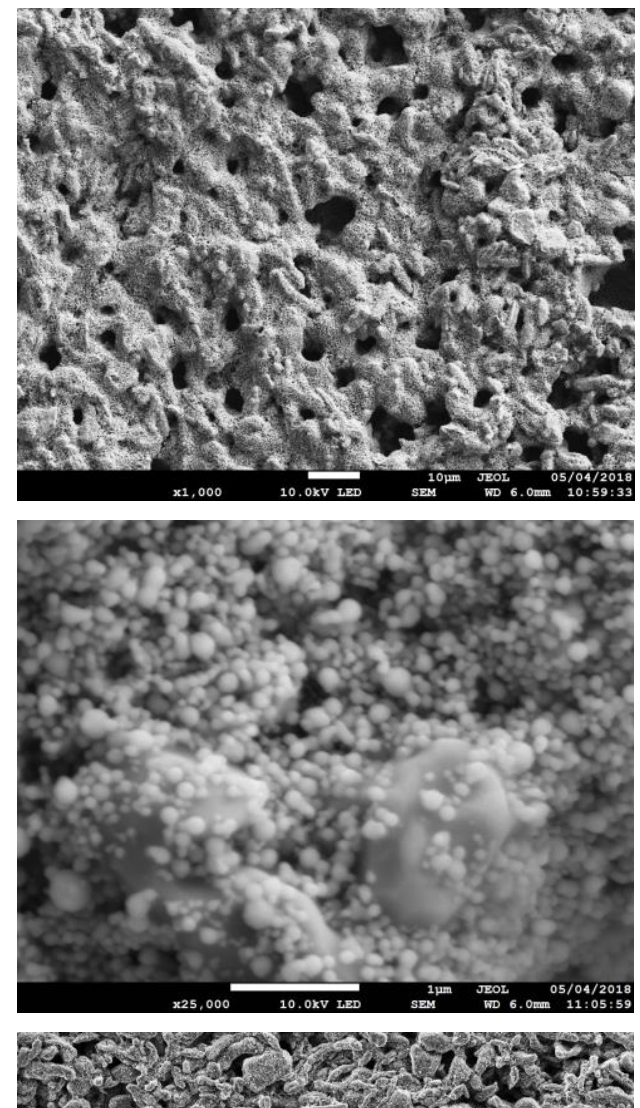
2150.0.

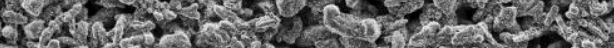

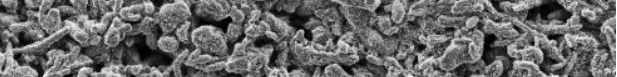

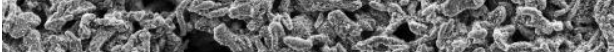
-1. 1 .

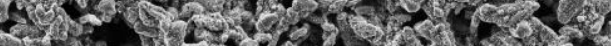

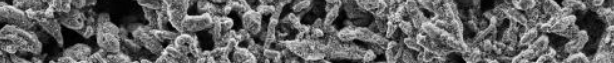

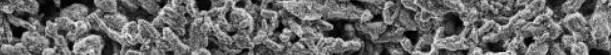
n. तn

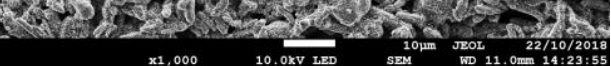

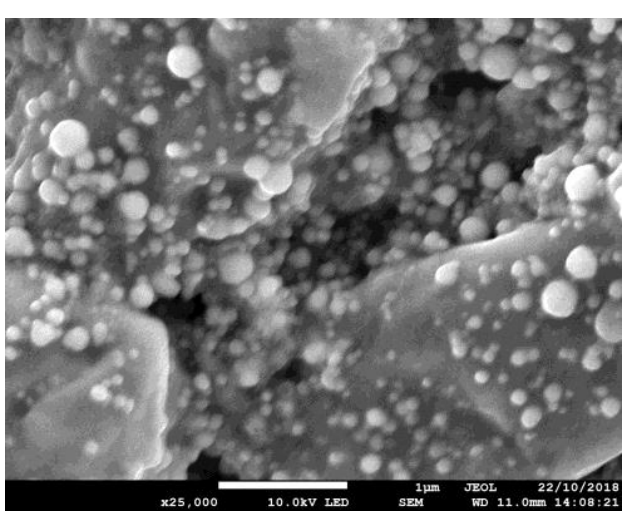

Figure 3. 20 SEM images of the dry and $\mathrm{N}_{2}$ sintered film 


\subsubsection{1: SEM examination Formic acid sintering condition}

Thermally sintered films with the presence of formic acid also introduced a significant enhancement in uniform morphology throughout the whole printed line, Figure 3.21.

Dry

(a)

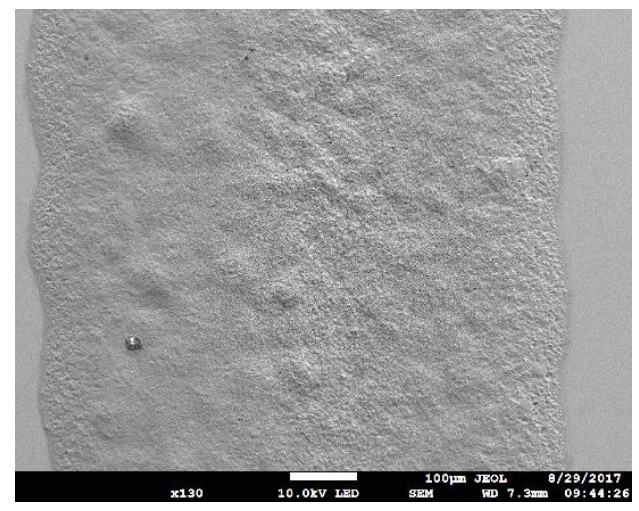

(b)

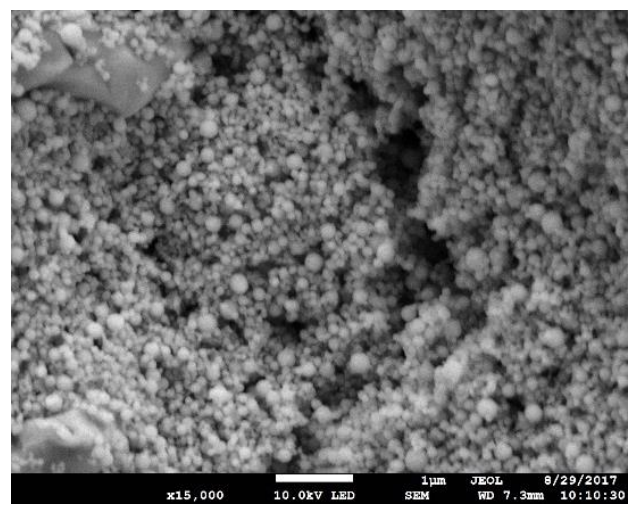

(c)

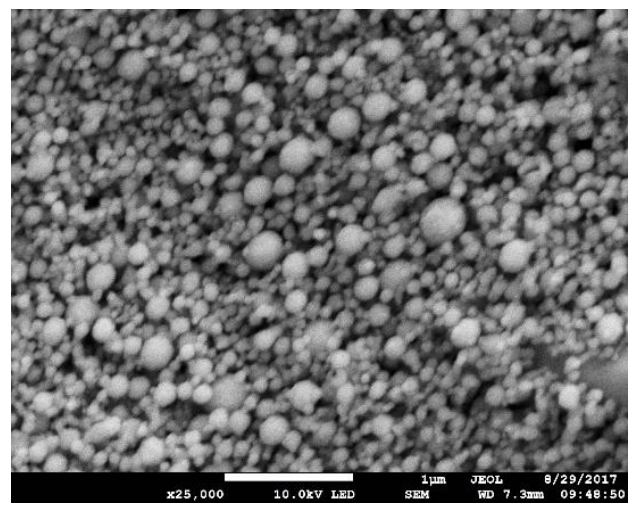

Formic acid Sintering
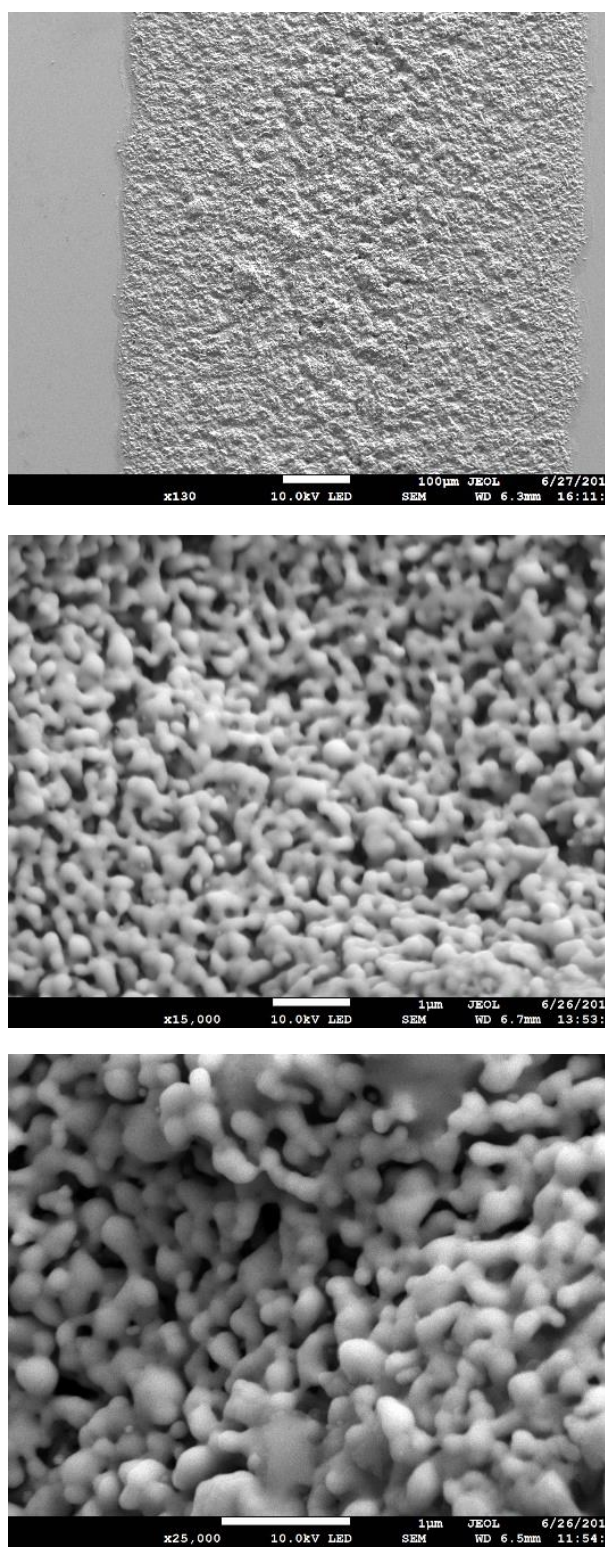

Figure 3. 21 SEM images of the dry and formic acidsintered films $100 \mathrm{~N}$

When sintered in formic acid, the individual nano-particles neck and merge to form a continuous globular network of particles, although voids remain present in the film, the necking of the individual particles resulted from the formic acid atmosphere has led to enhancement in conductivity by creating a significant current path. There is no immediate visual difference in the SEM images between the behaviour under a formic acid compared to the nitrogen environment for the $100 \mathrm{~N}$ ink. 
Figure 3.22 illustrates the SEM images of 50N50m and 20N80M sintered with the presence of formic acid atmosphere.

(a)

$50 \mathrm{M} 50 \mathrm{~N}$

(b)

50N50M

(c)

20N80M

(d)

20N80M
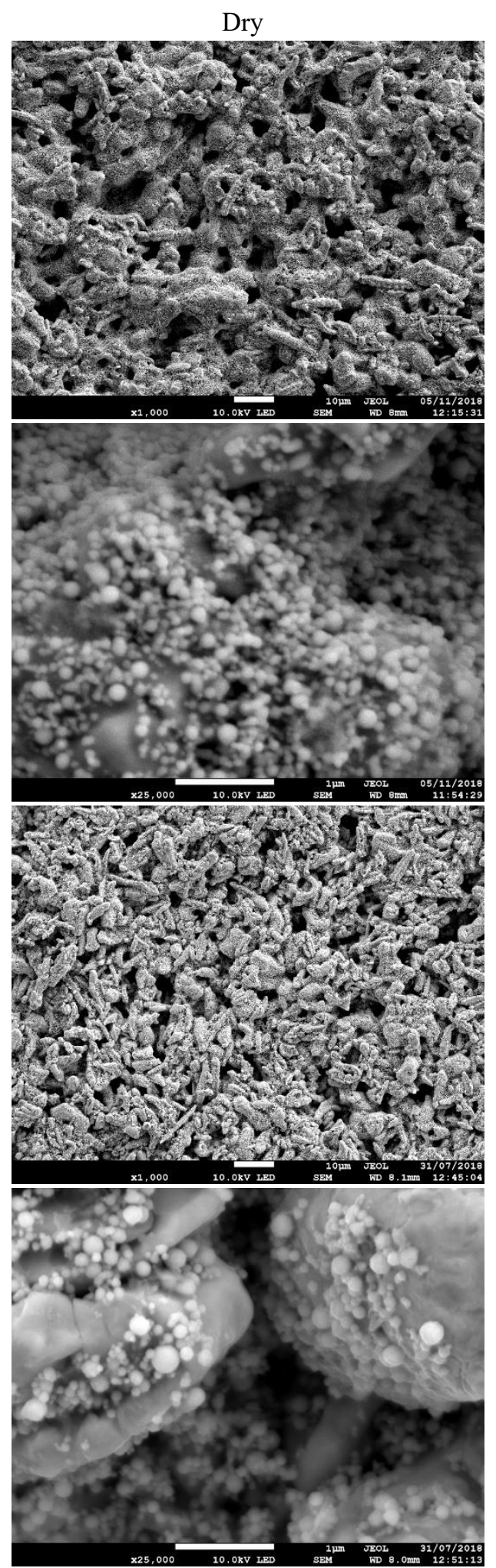

Figure 3. 22 SEM images of the dry and formic sintered films (50N50M and $20 \mathrm{~N} 80 \mathrm{M}$ )
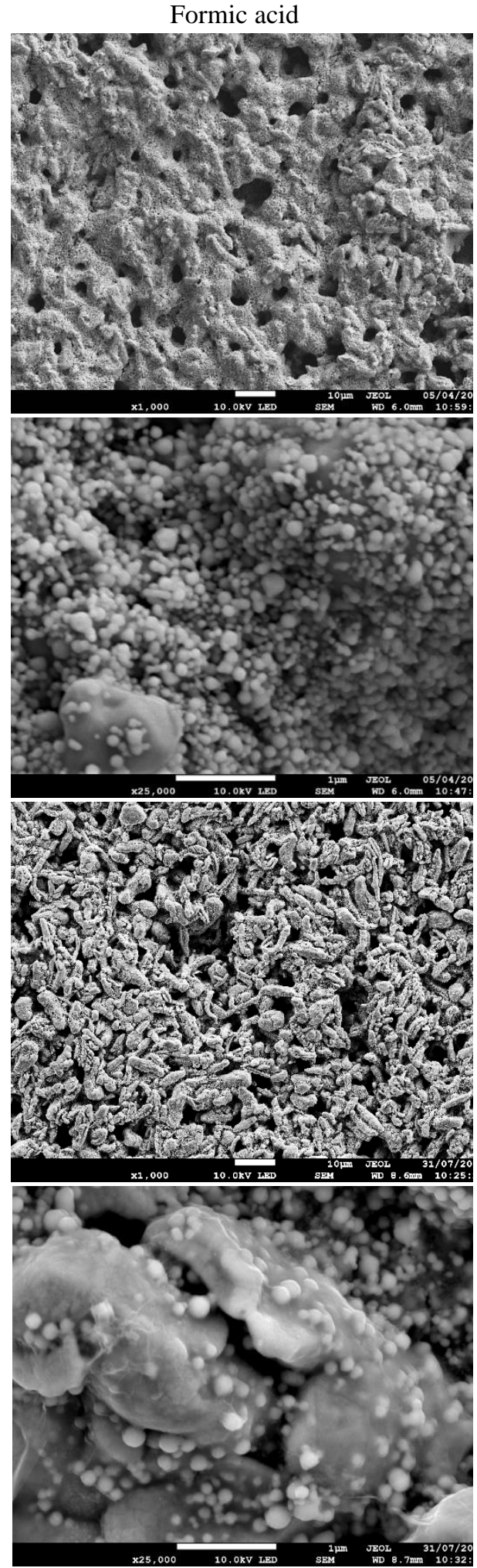
The SEM images displayed in Figure 3.22 represent the sintered samples at $275^{\circ} \mathrm{C}$ for 30 minutes which results in the lowest film resistance. On a macroscopic scale, some changes in the surface smoothness are observed, Figure 3.22 (a) and (c). As with the nitrogen atmosphere, there is some evidence of nano-particle necking into the larger particles, but each nano-particle remains as a distinct spherical particle. The necking between the nano and micro particles achieved will have led to some enhancement in conductivity by creating a significant current path.

\subsubsection{2: $\mathrm{SEM}$ examination $\mathrm{H}_{2} / \mathrm{N}_{2}$ sintering condition}

When $\mathrm{H}_{2} / \mathrm{N}_{2}$ condition applied, there is printed copper lines shows significant changes in the surface morphology compared to the dry only film, Figure 3.23.

(a)
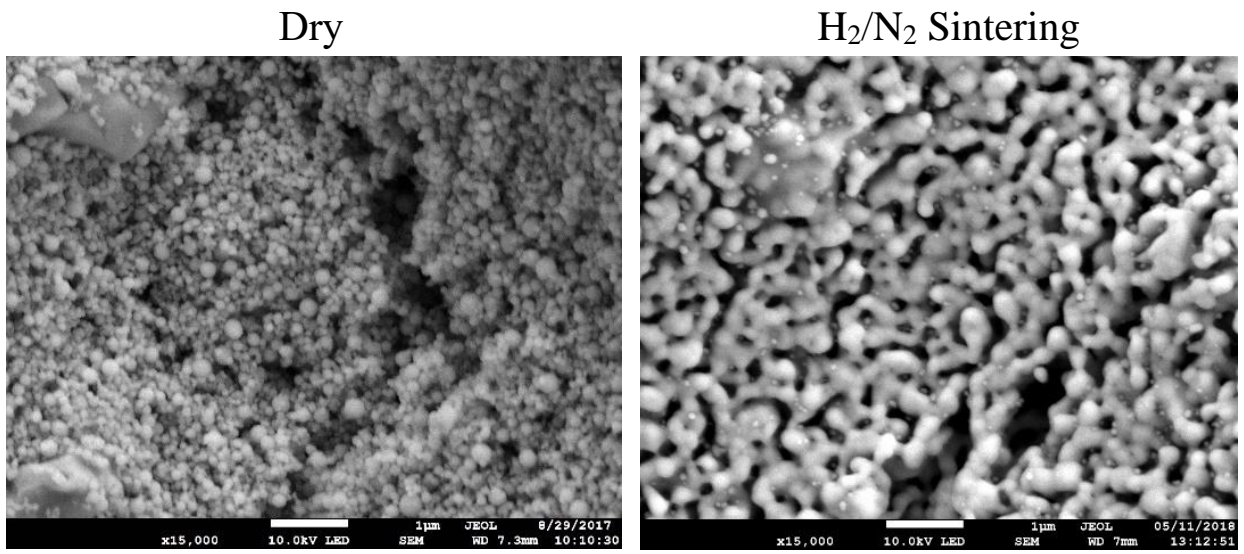

(b)
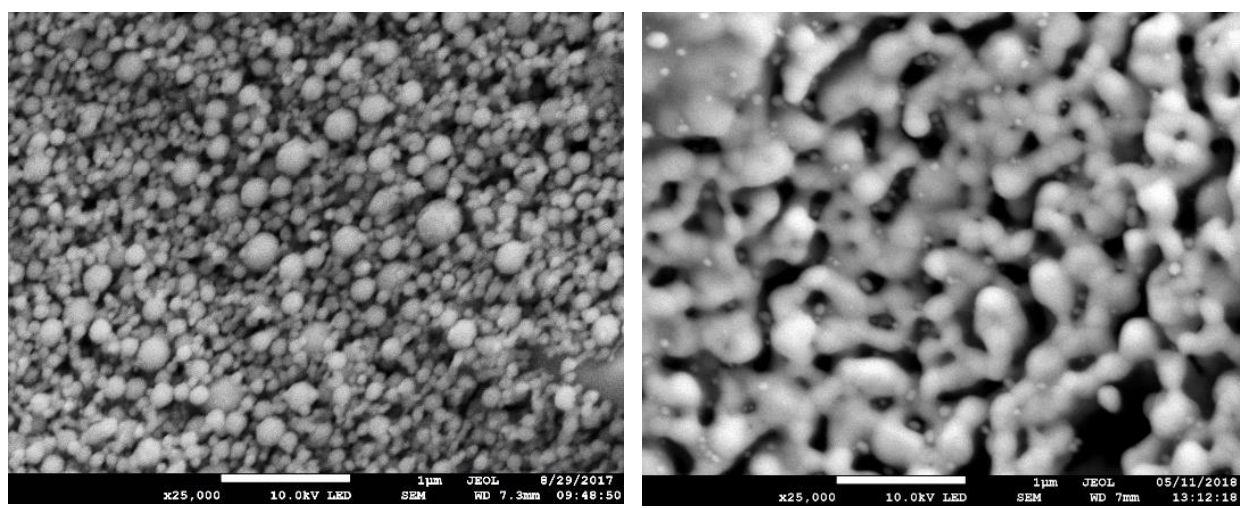

Figure 3. 23 SEM images of the dry and $\mathrm{H}_{2} / \mathrm{N}_{2}$ sintered films $100 \mathrm{~N}$

The individual nano-particles exhibit melting and merge at the interface between the particles, indicating a reasonable degree of sintering. Similar structures are obtained 
from sintered silver nano ink [147], [113]. This particle network allows efficient charge transfer through the film. There is some intrinsic porosity in the film which has formed as a result of necking of the particles and the removal of the binder between the particles.

Figure 3.24 illustrates the SEM images of $50 \mathrm{~N} 50 \mathrm{M}$ and $20 \mathrm{~N} 80 \mathrm{M}$ with the $\mathrm{H}_{2} / \mathrm{N}_{2}$ condition. The 50M50 N ink exhibits a structure where individual nano-particles are bound intimately to the surface and between the micro particles, Fig. 3.24 a, but the nano-particles between the micro particles primarily remain as discrete spherical particles with minimal inter particle sintering. At the lowest nano-particle concentration, the nano-particles appear as discrete "nodules" which are bound to the micro particles which are in intimate contact with each other, Fig. 3.24 b.
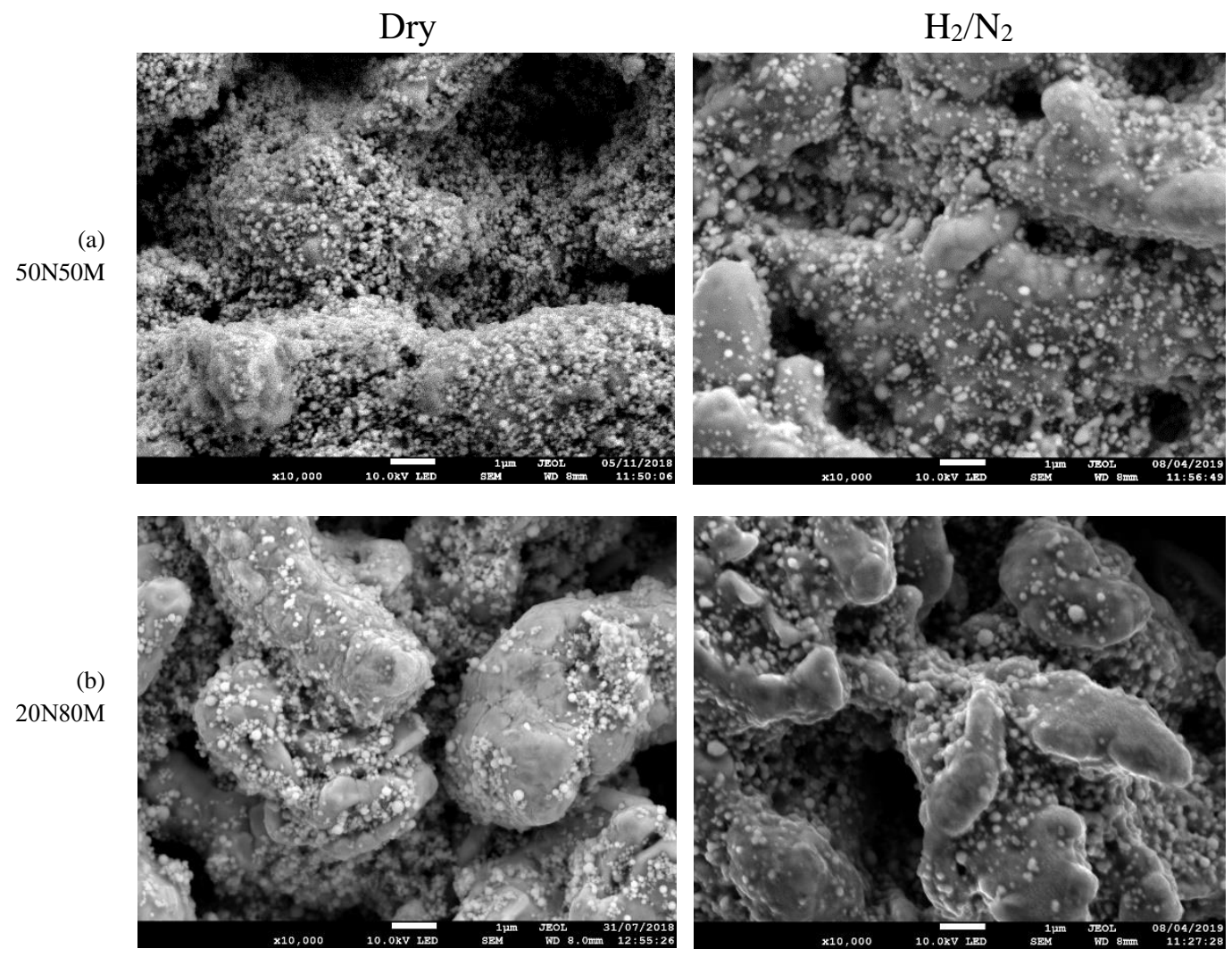

Figure 3. 24 SEM images of the dry and $\mathrm{H}_{2} / \mathrm{N}_{2}$ sintered films (50N50M and $20 \mathrm{~N} 80 \mathrm{M})$ 


\subsubsection{X-ray diffraction (XRD) Thermal sintering}

X-ray diffraction was employed to examine the structural and compositional properties of the ink and also used to investigate the sintered films, depending on crystal growth of the copper grain structures [82]. X-ray diffraction for the examined printed copper film shows that diffraction peaks with strong intensities appear at corresponding $2 \Theta 111,200$ and 220, this means the copper is formed with some oxidation. The XRD results of all three inks display in Figure 3.25.

(a)

$100 \mathrm{~N}$

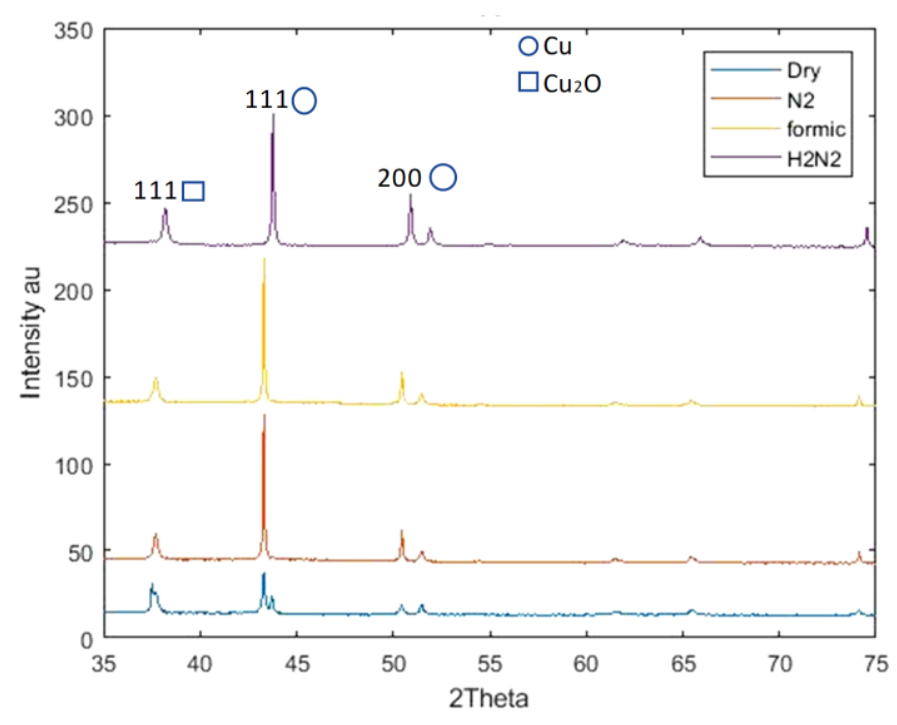

(b)

$50 \mathrm{~N} 50 \mathrm{M}$

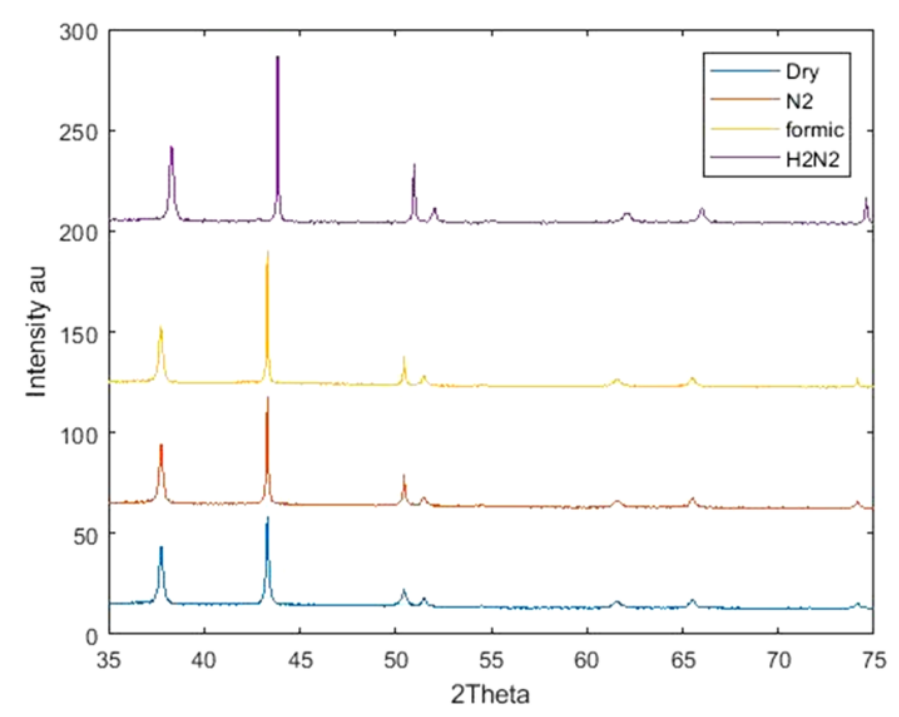


(c)

20N80M

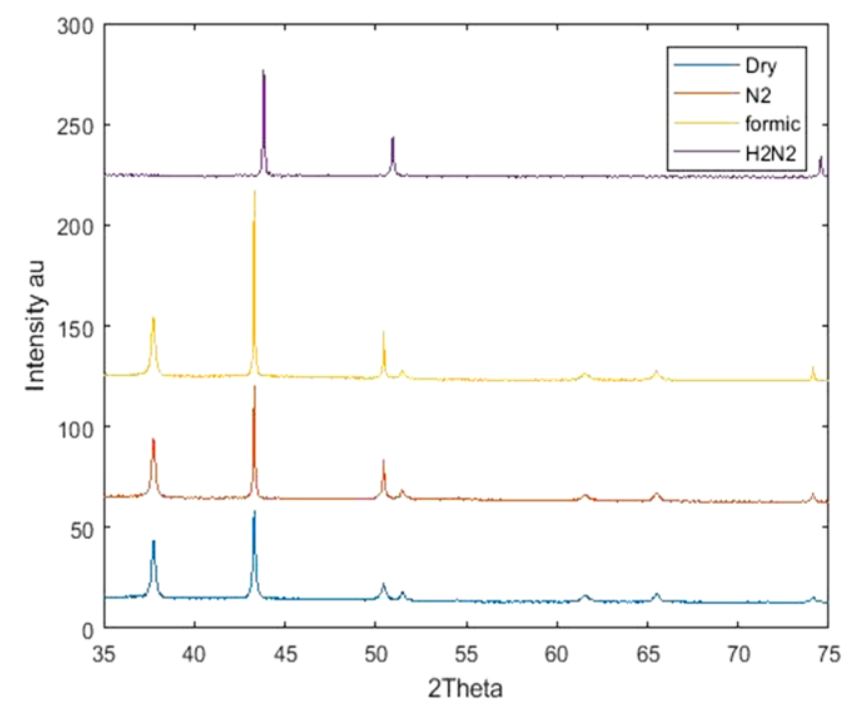

Figure 3. 25 XRD comparison of each film for each ink in a dry, $\mathrm{N}_{2}$, Formic acid and $\mathrm{H}_{2} / \mathrm{N}_{2}$

The X-ray diffraction is displayed for the printed copper film using $100 \mathrm{~N}$ ink with a comparison of each film for each ink in a dry, $\mathrm{N}_{2} 100 \mathrm{~N}$ ink sintered for $60 \mathrm{~min}$ at 200 ${ }^{\circ} \mathrm{C}$, Formic acid for $60 \mathrm{~min}$ at $200{ }^{\circ} \mathrm{C}$ and $5 \% \mathrm{H}_{2} / \mathrm{N}_{2}$ for $60 \mathrm{~min}$ at $200{ }^{\circ} \mathrm{C}$. The nanomicro ink sintered at $275^{\circ} \mathrm{C}$ for 30 minutes. These represent the best performing materials in terms of lowest resistance.

XRD examination of dry printed copper films, Fig. 3.34a-c shows the three characteristic peaks at $43.2^{\circ}, 50.3^{\circ}$, and $74.1^{\circ}$ indicative of (111), (200), and (220), respectively. These characteristic peaks confirm that a face-centered cubic (FCC) copper phase is present. There is also evidence of the $\mathrm{Cu}_{2} \mathrm{O}$ phase at $36.6^{\circ}$ which indicates that some oxidation has occurred during the drying process for all three inks [32]. When sintered for an hour in nitrogen environment, all materials are dominated by the presence of the $\mathrm{Cu}$ peaks, although in case the $\mathrm{Cu}_{2} \mathrm{O}$ peak remains. The XRD spectra for the formic acid environment provide similar spectra to the nitrogen sintering environment which suggests that the $\mathrm{Cu}_{2} \mathrm{O}$ which is present in the dried sample does not undergo significant reduction during the formic acid sintering process. When sintered under a $5 \% \mathrm{H}_{2} / \mathrm{N}_{2}$ environment, the $100 \mathrm{~N}$ and $50 \mathrm{~N} 50 \mathrm{M}$ material continue to contain $\mathrm{Cu}_{2} \mathrm{O}$, but this is absent for microparticle dominated ink, 20N80M Figure 3.25-c. This suggests that the reducing conditions are conducive to the reduction of the $\mathrm{Cu}_{2} \mathrm{O}$ to $\mathrm{Cu}$, particularly as the temperature increases [148]. 
The XRD examinations show that the $20 \mathrm{~N} 80 \mathrm{M}$ sintered with $\mathrm{H}_{2} / \mathrm{N}_{2}$ are firmly formed copper peak with significant stability against oxidation Figure 3.34-c. These characteristic peaks supported by the SEM images confirmed the formation of Facecantered cubic(FCC), forming a copper phase with less copper oxide or any other impurities [16]. This suggests the particles have a preferential growth and agglomerated along the (111) and (200) direction. The XRD results as intensity peaks were more definite with using $100 \mathrm{~N}$, and this is a reflection of the higher nanoparticles content in this material. Diffraction is sensitive to the grain structure size of the material examined [140].

\subsection{7: Stability test after Thermal sintering under reducing atmosphere}

Since oxidation is a source of changes in printed film resistance, samples were selected and measured immediately after sintering and then those samples were measured on a weekly basis when stored under a nominal environment of $21{ }^{\circ} \mathrm{C}+/-1{ }^{\circ} \mathrm{C}, 60 \% \mathrm{RH}$ $+/ 10 \%$.

In each instance, the sample sintered for 30 minutes was chosen as all samples were blemish free at this sintering time. For the $100 \mathrm{~N}$ ink, there is a slight increase in resistance over time, and in each instance, this change is most considerable at lower sintering temperatures, Figures 3.26 (a)-(c). Of the three materials, the 50N50M material exhibits the most significant ageing effect, Figures $3.26(\mathrm{~d})-(\mathrm{g})$. There is an initial rise in resistance over the first week, followed by smaller increments over subsequent weeks, and this behaviour is consistent for all sintering temperatures. For the $20 \mathrm{~N} 80 \mathrm{M}$ material exhibit minimal resistance increase throughout the measurement, inferring a very stable printed film, Figures 3.26 (h)-(j).

These results bode well in terms of the longevity of the circuits during the manufacturing process and use. Still, it has the added benefit that the impact of any time delays between sample manufacture and characterization is limited. Thus, confidence levels in the characterization methods are increased. 


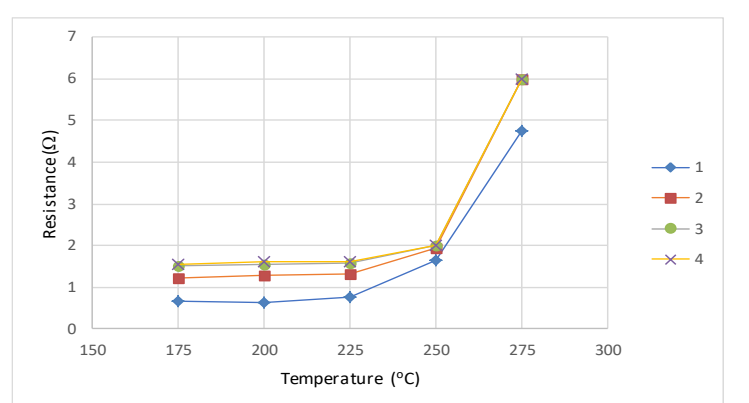

(a) $100 \mathrm{~N}-\mathrm{N}_{2}$

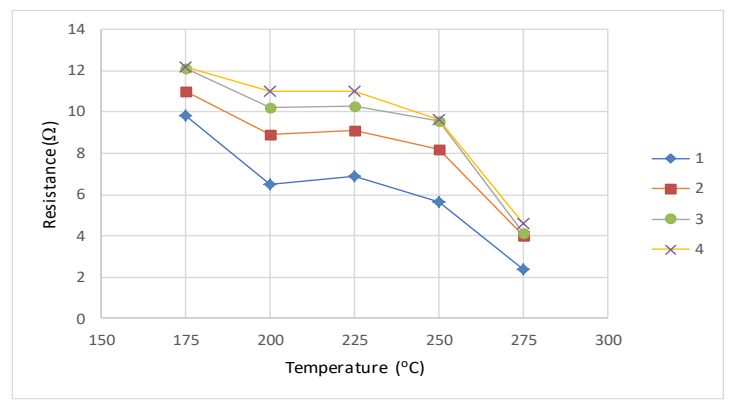

(e) $50 \mathrm{~N} 50 \mathrm{M}-\mathrm{N}_{2}$

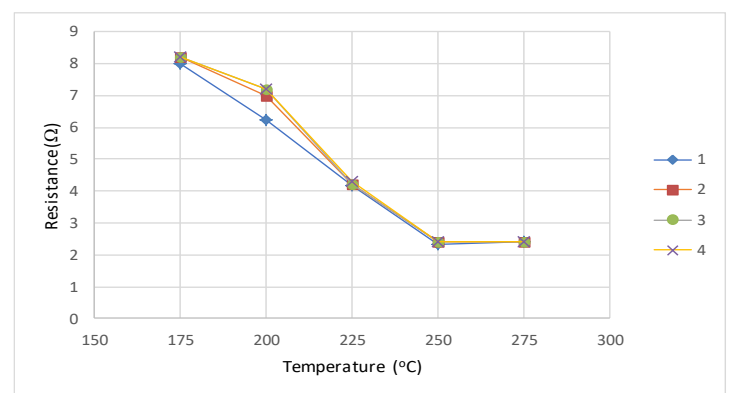

(h) $80 \mathrm{~N} 20 \mathrm{M}-\mathrm{N}_{2}$

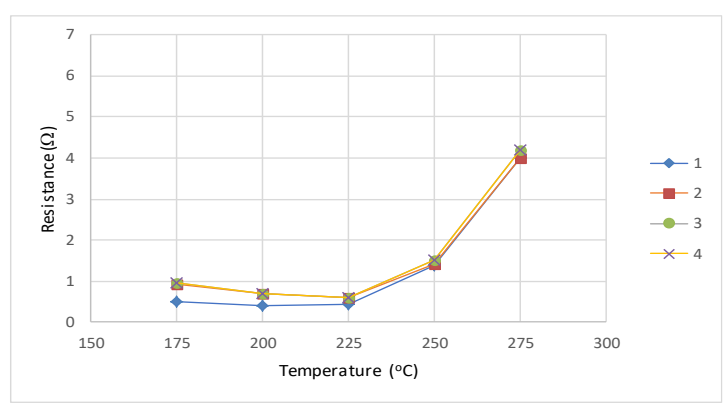

(b) $100 \mathrm{~N}$ - Formic acid

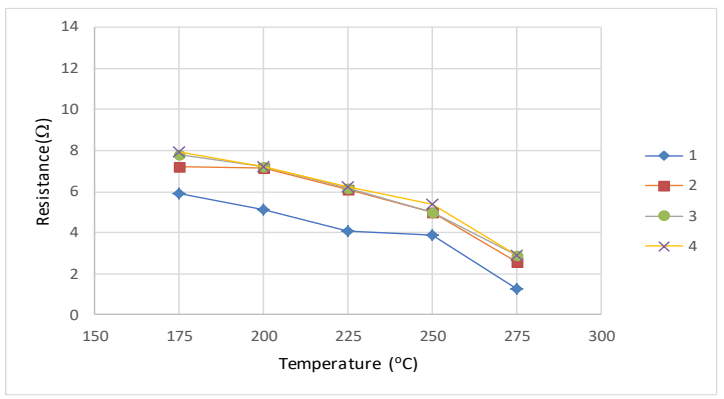

(f) $50 \mathrm{~N} 50 \mathrm{M}$ - Formic acid

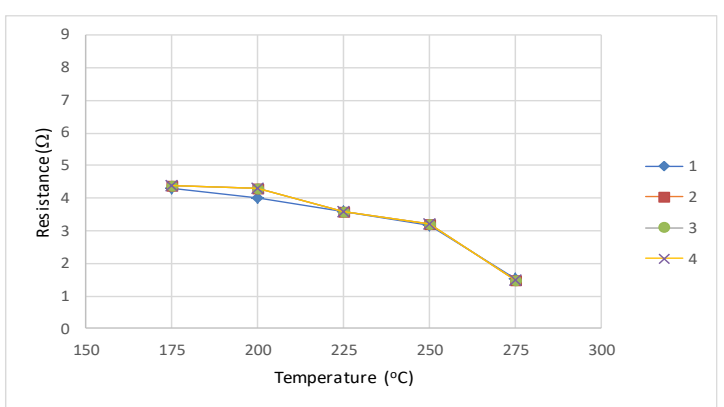

(i) $80 \mathrm{~N} 20 \mathrm{M}$ - Formic acid

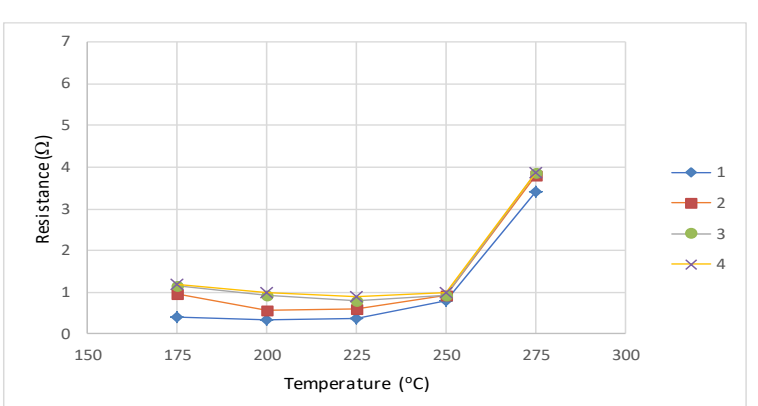

(c) $100 \mathrm{~N}-5 \% \mathrm{H}_{2} / \mathrm{N}_{2}$

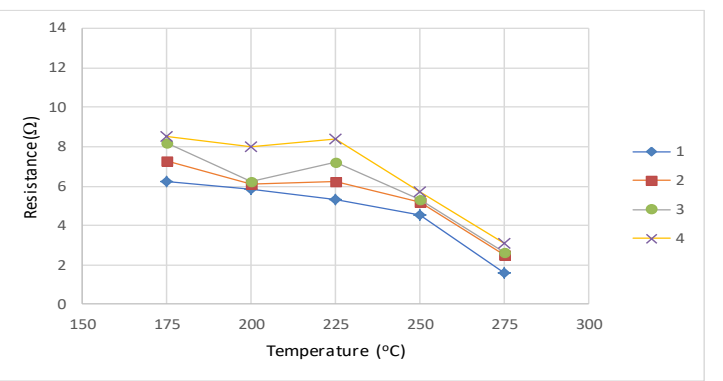

(g) $50 \mathrm{~N} 50 \mathrm{M}-5 \% \mathrm{H}_{2} / \mathrm{N}_{2}$

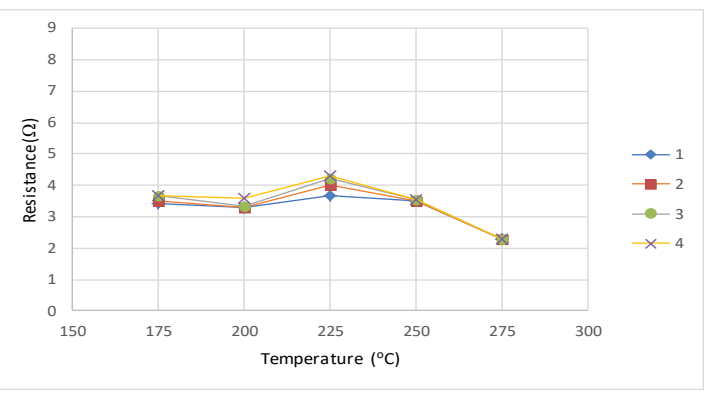

(j) $80 \mathrm{~N} 20 \mathrm{M}-5 \% \mathrm{H}_{2} / \mathrm{N}_{2}$

Figure 3. 26 Line resistance change over 4-week interval (labelled 1-4) for the three materials sintered at 30 minutes in each sintering environment. 


\section{5: Summary}

This chapter introduced the impact of the thermal sintering environment on the quality of the screen-printed copper material studied. The overall benefit of sintering in a reducing atmosphere produced by formic acid compared to a nitrogen environment is minimal. Thus, given the relative ease by which a nitrogen environment can be achieved in a typical production environment, the more complex formic acid-reducing environment is not recommended. The optimum sintering conditions (for minimum sheet resistance) for each material with relative resistivity to copper at $\left(\rho_{0}=1.68 \times 10^{-8}\right.$ $\Omega \mathrm{m})$ are summarized in Table 3.3.

Table 3. 4 Optimum conditions and resultant sheet resistance and relative resistivity for each ink and atmosphere (Relative resistivity to copper at $\rho_{0}=1.68 \times 10^{-8} \Omega \mathrm{m}$ )

\begin{tabular}{|c|c|c|c|c|c|c|c|c|c|c|c|c|}
\hline Condition & \multicolumn{4}{|c|}{$\mathbf{N}_{2}$} & \multicolumn{4}{|c|}{ Formic acid } & \multicolumn{4}{|c|}{$5 \% \mathrm{H}_{2} / \mathrm{N}_{2}$} \\
\hline ink & $\begin{array}{l}\mathrm{R}_{\mathrm{s}} \\
\mathrm{m} \Omega / \square\end{array}$ & $\rho / \rho_{0}$ & $\begin{array}{l}\text { topt } \\
\text { (min) }\end{array}$ & $\begin{array}{l}\mathrm{T}_{\mathrm{opt}} \\
\left({ }^{\circ} \mathrm{C}\right)\end{array}$ & $\begin{array}{l}\mathrm{R}_{\mathrm{s}} \\
\mathrm{m} \Omega / \square\end{array}$ & $\rho / \rho_{0}$ & $\begin{array}{l}\text { topt } \\
\text { (min) }\end{array}$ & $\begin{array}{l}\mathrm{T}_{\text {opt }} \\
\left({ }^{\circ} \mathrm{C}\right)\end{array}$ & $\begin{array}{l}\mathrm{R}_{\mathrm{s}} \\
\mathrm{m} \Omega / \square\end{array}$ & $\rho / \rho_{0}$ & $\begin{array}{l}\text { topt } \\
\text { (min) }\end{array}$ & $\begin{array}{l}\mathrm{T}_{\mathrm{opt}} \\
\left({ }^{\circ} \mathrm{C}\right)\end{array}$ \\
\hline $100 N$ & 0.74 & 7.3 & 60 & 225 & 0.89 & 6.3 & 60 & 225 & 0.49 & 5.8 & 60 & 225 \\
\hline $50 N 50 M$ & 3.99 & 28 & 30 & 250 & 4.16 & 15 & 30 & 250 & 2.34 & 12 & 30 & 250 \\
\hline $20 N 80 M$ & 1.99 & 29 & 30 & 275 & 1.65 & 19 & 30 & 275 & 1.61 & 16 & 30 & 275 \\
\hline
\end{tabular}

There is a clear and consistent benefit of using the more controlled reducing atmosphere produced by the $5 \% \mathrm{H}_{2} / \mathrm{N}_{2}$ mixture. As the proportion of micro particles increases, there is an increase in the sintering temperature required. Overall, the measured sheet resistance is between 2.2 to 8 times higher for the micro nano blends inks than that obtained pure nano material ink. In comparison to the base resistivity of copper, the lowest obtainable resistivity is around six times that of copper for the $100 \mathrm{~N}$ ink. The micro-nano blended materials have a higher material resistivity, between 12 and 28 times that of base copper with lower resistivity under reducing conditions. 


\subsection{Discussion}

The relatively poor performance of the 50N50M blend (highest sheet resistance, the largest ageing effect) suggests that there is an optimum blending ratio of nano/micro particles, which balances the performance benefit of each constituent. It is postulated that the presence of an excessive quantity of micro particles disrupts the natural sintering tendency of the nano-particles as they are rapidly bound to larger surface area micro particles, leaving them open to oxidation. Where the nano-particles form a major contributor to the continuous conductive $(50 \mathrm{~N} 50 \mathrm{M})$ pathways, this impairs charge transfer through the particles, Figure 3.27. At lower concentrations of nanoparticles, a conductive path is ensured through microparticle contact, and thus the impact of any nano-particle oxidation is minimized. Evidence for this hypothesis can be seen in the absolute conductivity and in the increase in resistance seen with ageing, suggesting that there are some nano-particles which are not fully sintered which are subject to oxidation. While the short-term stability of the copper inks has been shown to be right when sintered under the correct conditions, further work should examine more extended exposure periods under harsher thermal and moisture conditions which the conductive materials may be subjected during the lifetime of a product.

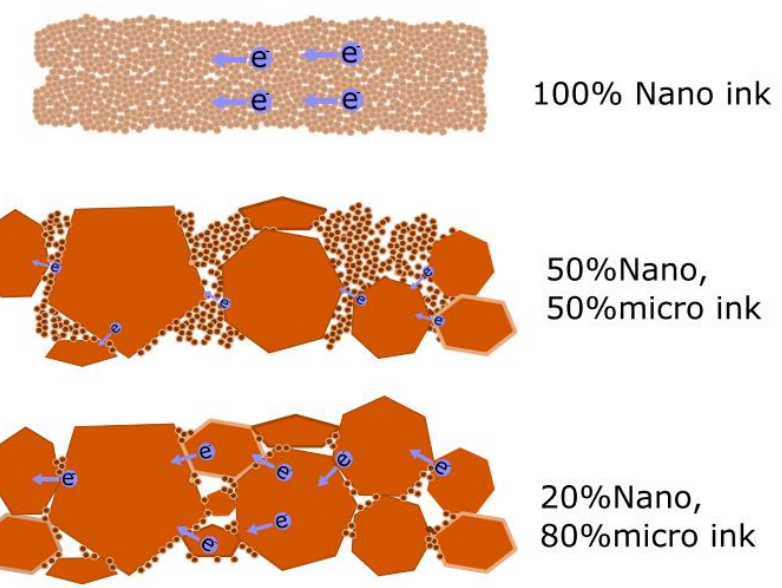

Figure 3.27 Charge transfer through the particles

If printed copper lines were to be used to enhance the conductivity of a transparent surface (e.g. for charge extraction in PV) then it should be noted that the copper / FTO contact resistance is the same order of magnitude as the resistance of line $30 \mathrm{~mm}$ long $600 \mu \mathrm{m}$ wide line (Figure $3.15 \& 3.17$ ). The interfacial resistance component would 
form a significant portion of the overall resistance. This is in contrast to that observed when copper deposition via magnetron sputtering [149], which would with FTO and copper having work functions of $4.4 \mathrm{eV}$ [150] and $4.7 \mathrm{eV}$ suggesting relatively unhindered charge transfer, Figure 3.28. This tends to indicate that the actual contact area and contact chemistry is significantly more complicated when using a printed ink. It also implies that any models for enhancing charge transfer through the use of printed mesh must take into account the contact resistance.

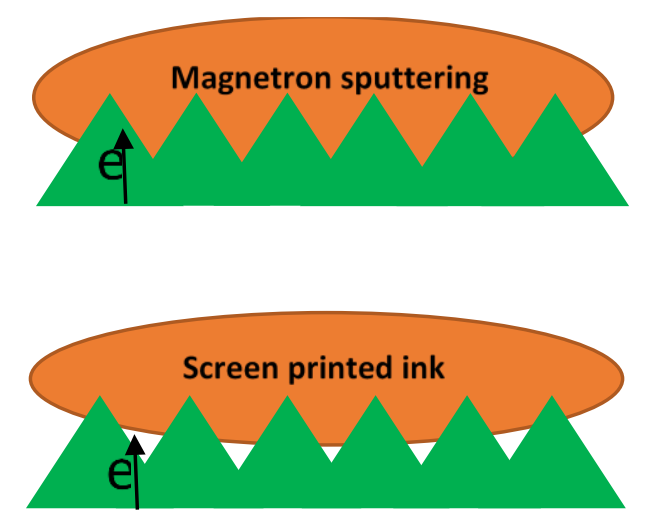

Figure 3. 28 Charge transfer between the electrode and the conductive film

\section{7: Conclusions}

There is the significant interaction between the particles size distribution, the thermal sintering regime, the sintering environment used and the subsequent characteristics of the final film when conductive copper inks are sintered on FTO coated glass substrates. The lowest resistances can be obtained using the pure nano-copper ink, and they also offer the advantage of a lower sintering temperature but a longer sintering time to achieve the lowest sheet resistance. Blending nano-particles with micro particles can be disadvantageous in that it prevents full consolidation and sintering of the nano-particles, allowing them to be partially oxidized during the sintering stage. From the initial main results and sintering process comparison, future chapters investigated how employing a new mix-material and structure will affect the conductivity performance of the screen-printed thick metal film. 


\section{Chapter 4: Photonic sintering}

\section{1: Introduction}

Pulsed light sintering is the fastest process that can be used to sinter copper ink. In addition to the fast sintering, it potentially sinters without causing any damage to the substrate and eliminates the need for reducing atmosphere to be employed [10]. The super-fast sintering process offers a means by which rapid conversion from discrete nano-micro particles to fully or partially sintered products. It has been shown to sinter silver nano-inks in less than 1-second achieving conductivities comparable to oven sintered materials [77].

Photonic sintering has potential for copper sintering [6] [151] [152] with benefits of photonic sintering are near instant sinter, with sintering times of seconds instead of minutes allowing higher material throughput and productivity. Potentially lower power usage as energy is used to raise the temperature of the printed copper film only and not the substrate [18]. More details in chapter 1.2 the literature review.

The literature, however, has not examined glass substrates, which have been coated with an FTO surface or a thick screen-printed film. The absorption of the FTO has the potential to act as an additional energy source at the boundaries of any feature. At the same time, the thick glass substrate provides an energy sink which may retard sintering. The thick film nature of the substrate provides an additional quantity of material which needs to be sintered and provides the other challenge of transferring the energy through the bulk to the core of the film. Thus, the study aimed to examine the feasibility of sintering a thick film of copper ink on an FTO glass substrate. The sintering operation range was identified by carrying an energy optimization study in 4.2 section.

\section{2: Optimization the PulseForge Energy}

The generation of light energy depends on the size of the capacitors and the voltage applied to that capacitor [111], [153], [154]. An initial optimization study was carried out to identify an operational range of photonic energy profile. An initial investigation study was carried out to see if the photonic curing could be used to both dry and sinter the wet ink. But this was rapidly discarded as a concept as the photonic process had 
minimal effect on the wet ink, even at its highest setting. The energy required to volatilize the solvent, the kinetic limitations of migration of the solvent through the wet ink to film surface were seen as mechanisms for this behaviour. Subsequently, all samples which was dried in the same manner as before the thermal sintering.

With the dried ink, a strategy was employed to obtain the best possible effect of the flashlight which could lead to a good conductivity range by applying a different number of pulses with variety of voltage which then enabled the estimation of the maximum, and the minimum power applied. Through, the refined pulse width, power and spacing the nominal optimum conditions were established for the ink/substrate combination. A single pulse did not provide enough energy input to sinter and as such a 3-10 pulse strategy was adopted after several trials carried out and according to the machine safety operation supplied by the manufacturer. Figure 4.1 shows several examples of the relationship between the number of pulses or voltage applied to the results of energy. The ten pulses setting was the primary condition used on sintering $100 \mathrm{~N}, 50 \mathrm{~N} 50 \mathrm{M}$ and $20 \mathrm{~N} 80 \mathrm{M}$ ink, as the most promising results obtained at this setting.

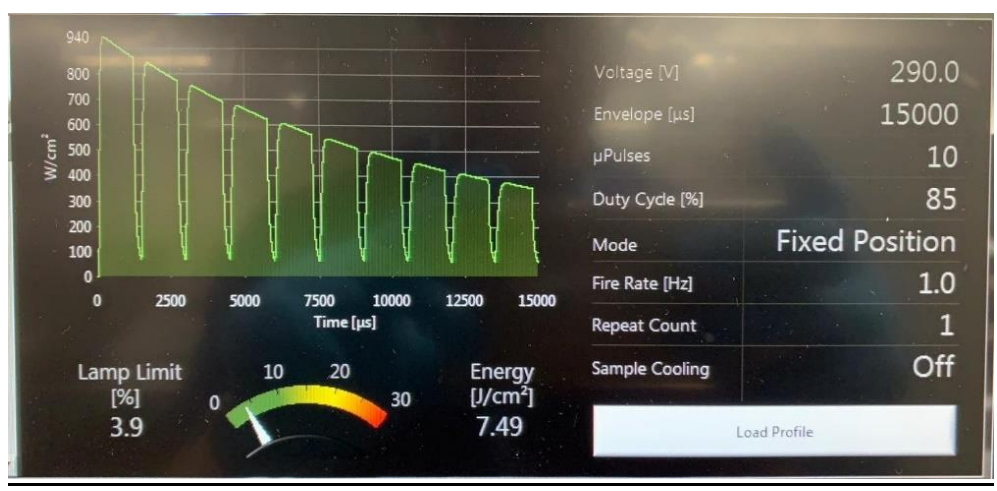

10 Pulses

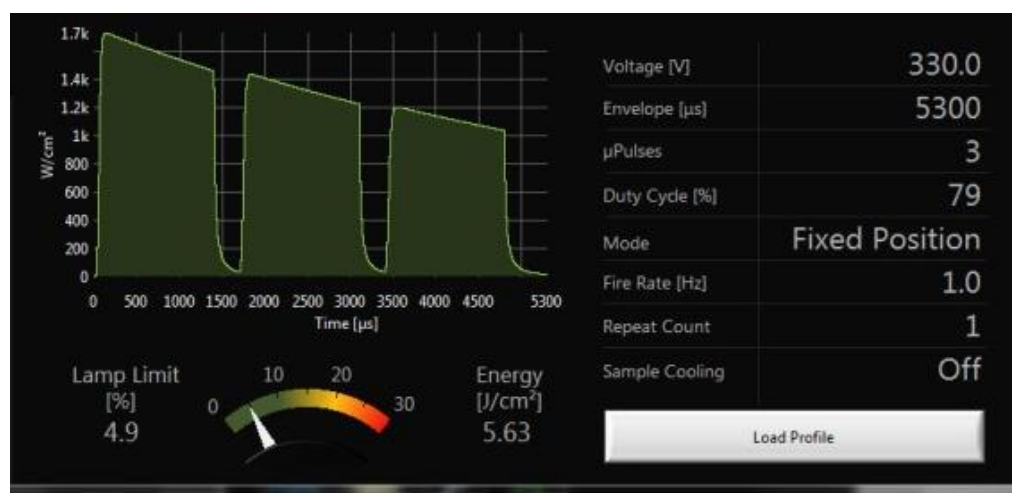

3 Pulses

Energy:

$5.63 \mathrm{~J} / \mathrm{cm}^{2}$

Figure 4. 1 Energy - pulses relationship 
The applied voltage considered is the primary control parameter which then dictates the energy resulted, as charging the capacitor to a higher voltage will lead to a longer lighting pulse when the capacitor discharges [112]. The time of exposure for discharge is termed the envelope. The discharge characteristic follows an exponential decay and this discharge profile can be altered by timing the discharge to create pulses of light. The number of the pulses and the length of each (or duty cycle) can be considered as secondary control parameters to dictate the energy, applying higher number of pulses with the same voltage charge will lead to a shorter pulse [155].

However, multi-light pulses can be applied with the total energy output of each pulse can be determined by the voltage and duration time employed. Table 4.1 and Figure 4.2 illustrates a simulated pulse forge power output to be compared to the energy obtained on the optimization study by applying different voltage and number of pulses. This simulation was created to allow understanding of the control process and to allow some level of prediction of the likely impact of longer exposure times on the intensity.

Given an initial power (derived from the applied voltage), an exponential fit was applied which estimate the power according to the time dependence of a physical quantity $\mathrm{P}$ is given by:

$$
P=P_{0} e^{-\alpha t}
$$

Eq4.1

where $P_{0}$ is the initial amount at time $t=0$, and $a$ is the growth rate

By iterative modelling $\alpha$ was found to have a value of around $7.6 \times 10^{-5}$ and this rate decay is the same, irrespective of the duty cycle; the charge being "dumped" internally through a load resistor when not passing through the lamp. 
Table 4. 1 Energy - simulated PulseForge data

\begin{tabular}{|l|l|l|}
\hline Envelope & 15000 & 5300 \\
\hline Number of pulses & 10 & 3 \\
\hline Duty Cycle \% & $85 \%$ & $79 \%$ \\
\hline Section width & 1500 & 1767 \\
\hline Pulse width $(\mu \mathrm{s})$ & 1275 & 1396 \\
\hline Energy obtained $\left(\mathrm{J} / \mathrm{cm}^{2}\right)$ & 8.88 & 5.63 \\
\hline
\end{tabular}

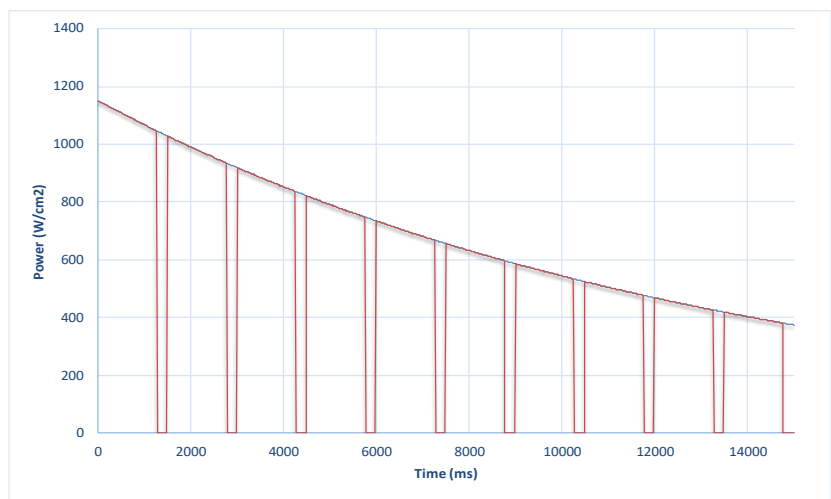

10

Pulses

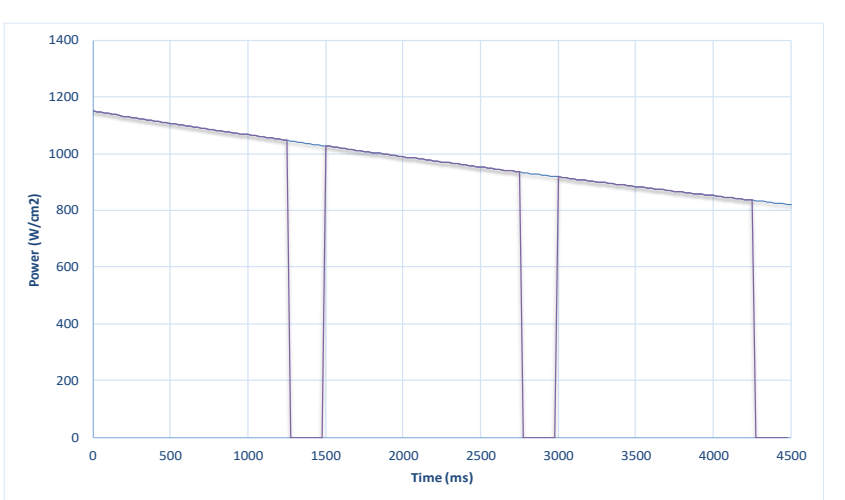

pulses

Figure 4. 2 Energy - simulated PulseForge data

In practice the transition between the lamp in an "on" and "off" state is more gradual than that predicted by the model where transitions are sudden. This is associated with lamp heating and cooling time.

As there were no literature available and no guidelines available for the sintering, a trial and error approach were initially required. During this period, samples were exposed, visually examined, measured, and stored with the measurements guiding the next combination of parameters which might produce improved performance (the thermal sintering in chapter 3 acting as guide to best achievable). 
This trial and error approach were necessary, but also wasteful in the number of samples required. At each condition, the test was "destructive" in that even if the sample showed no visible sign of sintering, it would not be used as a test sample again. Typically, around 80 samples were required to focus in on a set of conditions which could be considered optimized. This issue limited the number of operational parameters which could be investigated thoroughly, particularly in light of the limited supply of copper ink. During the initial studies, it was found that a multi short pulse of energy has better performance that a long energy burst. This could be attributed to the mechanism which can give time to the substrate to cool-down, leading to lower stress on the film interface. A consistent feature which was evident at both 3 and 10 pulses was that increasing the energy in the pulse initially reduces the resistance, but further increases lead to a catastrophic failure of the film. When applying three pulses, energy densities beyond $6.09 \mathrm{~J} / \mathrm{cm}^{2}$ resulted in cracking and destruction of the printed lines using the $100 \mathrm{~N}$ ink, Figure 4.3(a). With 10 pulses there is a reduction in line resistance up until a maximum energy $10.14 \mathrm{~J} / \mathrm{cm}^{2}$ after which further energy input results in sample destruction. Further additional pulses did improve the resistivity as the intensity (dictated by the remaining capacitor charge) had dropped below a level which resulted in sintering.

Photonic sintering presented a similar behaviour with the 50N50M ink, with minimum resistances being obtained at 6.1 and $14.2 \mathrm{~J} / \mathrm{cm}^{2}$ for the 3 and 10 pulse respectively, Figure 4.3 (b). This material was able to endure a higher energy $18.66 \mathrm{~J} / \mathrm{cm}^{2}$ before the film started to ablate. The tolerable operational window of the $50 \mathrm{~N} 50 \mathrm{M}$ ink was therefore wider than the $100 \mathrm{~N}$, with less risk of damage due to a small change in lamp output. The absolute line resistance of the $100 \mathrm{~N}$ ink is around a factor of 3 lower than that observed with the 50N50M ink. Due to the limited quantity of material available and the trial and error nature, the operational envelope of the $20 \mathrm{~N} 80 \mathrm{M}$ ink could not be investigated as widely. A 10-pulse strategy showed an operational window between 8.2 and $8.8 \mathrm{~J} / \mathrm{cm}^{2}$ where minimum resistance was obtained, Figure 4.3(c). Ideally it would have been possible to carry out the experiments with 3 pulses with the $20 \mathrm{~N} 80 \mathrm{M}$ ink, but material limitations quantities prevented this. 


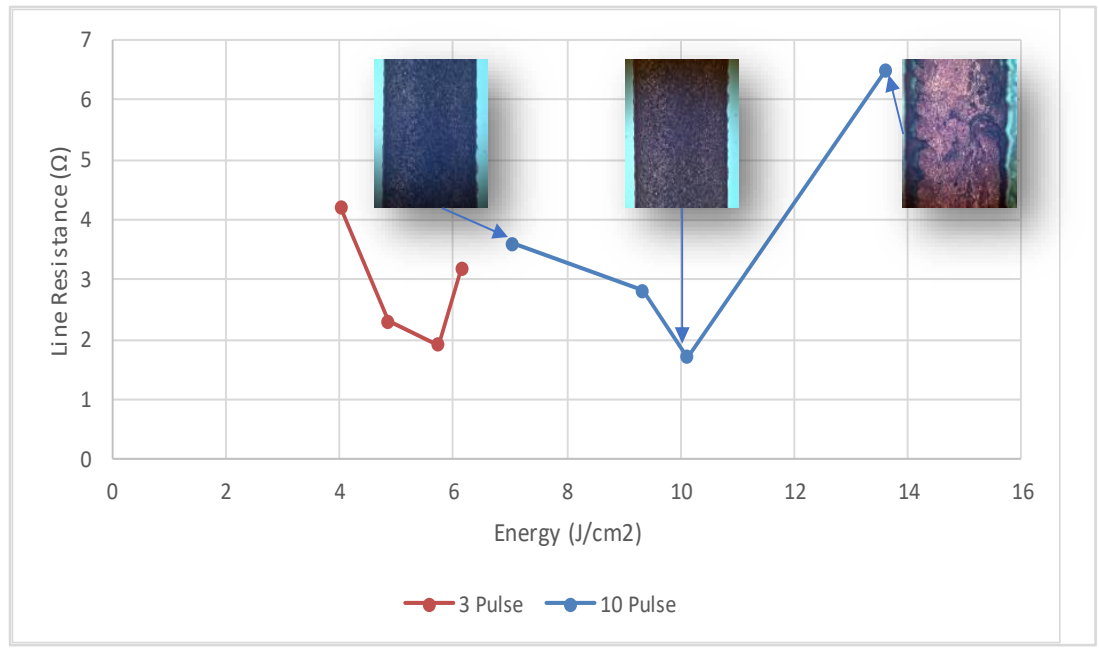

(a) $100 \mathrm{~N}$

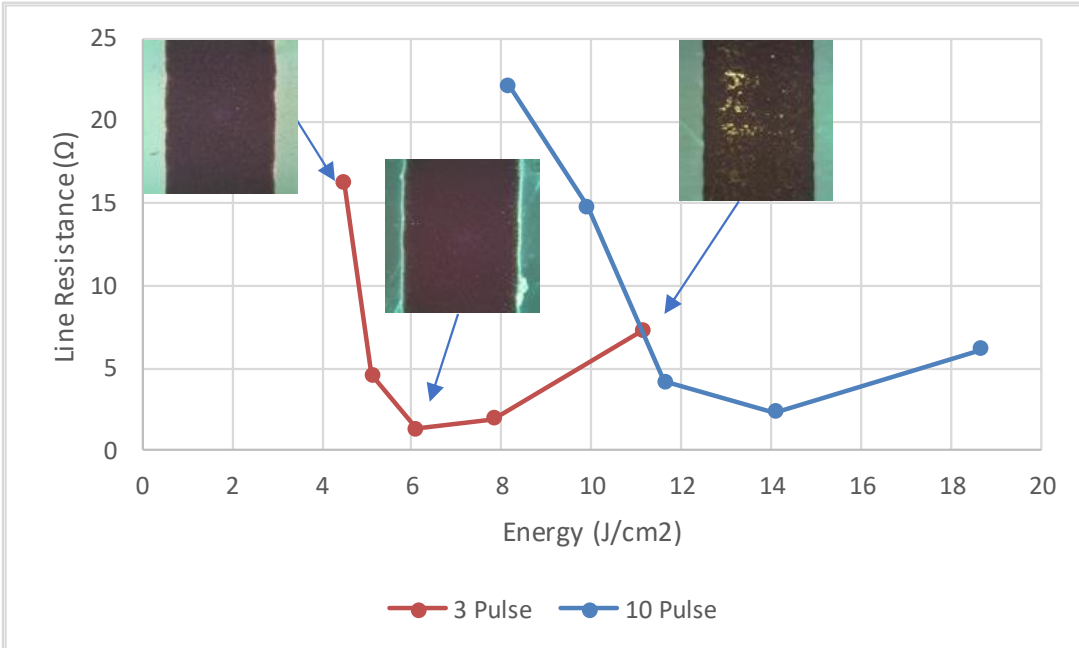

(b) $50 \mathrm{~N} 50 \mathrm{M}$

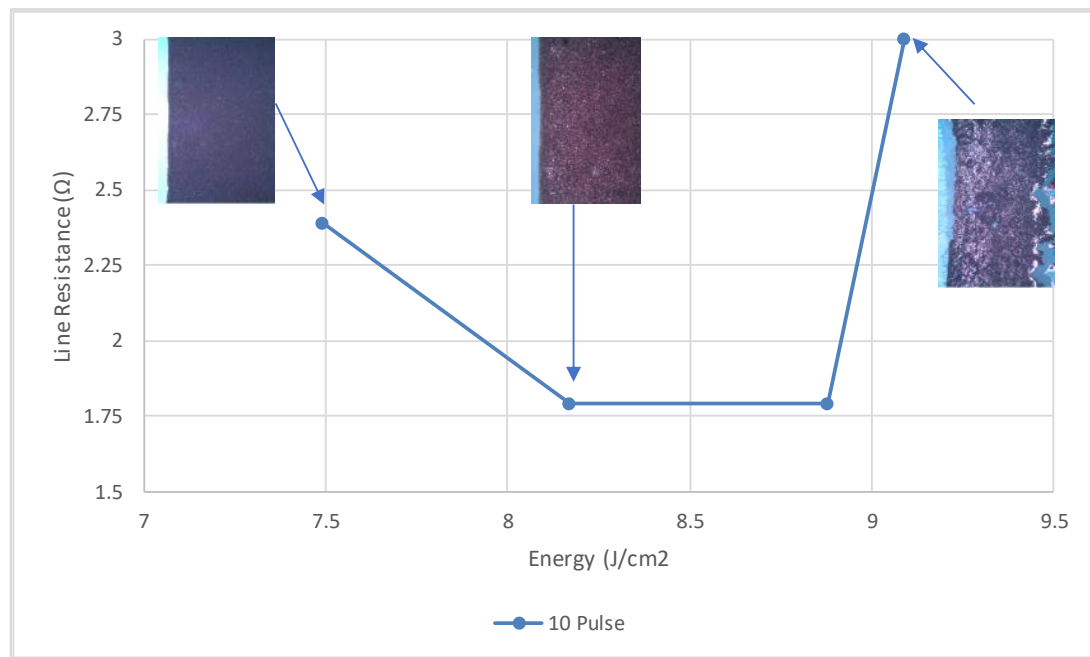

(c) $20 \mathrm{~N} 80 \mathrm{M}$

Figure 4. 3 Line resistance for the $600 \mu \mathrm{m}$ wide line for each ink under each photonic illumination regime. 


\subsection{Structure \& size}

Given that the surface absorption was the source of energy for the sintering process, then some changes in the optimum sintering conditions were expected for each line width as the ratio of surface area to bulk volume changes. This was experienced with finer structures being adversely affected by exposure to the photonic energy with areas exhibiting substrate delamination and physical damage, Figure 4.4. Thus, the photonic ideal sintering regime is a function of the structure size.

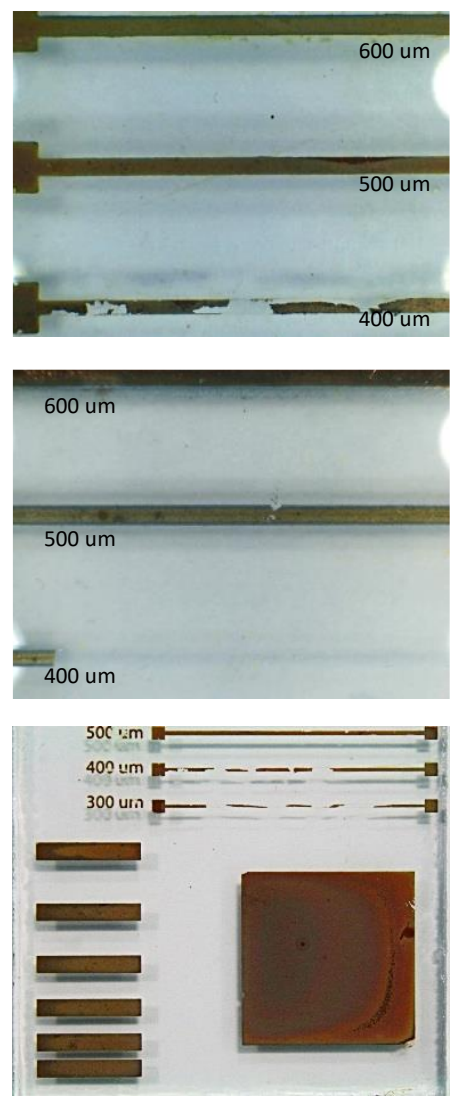

(a) Average damage on $400 \mu \mathrm{m}$ while $500 \mu \mathrm{m}$ and $600 \mu \mathrm{m}$ were sintered

(b) Severe damage on $400 \mu \mathrm{m}$, slight damage on $500 \mu \mathrm{m}$, sintered $600 \mu \mathrm{m}$

(c) Average damage on 300 and $400 \mu \mathrm{m}$, sintered $500 \mu \mathrm{m}$, partially sintered large printed objects

Figure 4. 4 Examples where the degree of sintering differs between adjacent features at different geometric sizes.

By normalizing the measured resistance to the minimum value obtained it is possible to determine any shift in the optimum energy required for minimum resistance and the tolerance Figure 4.5. All materials show an optimum value, below which there is minimal sintering and above which there is over-sintering with possible damage to the structures. The lower the number of pulses, the greater the impact in the change in resistance for a given change in energy delivered. This is evident from by examining the gradient of the lines from the point of minimum resistance for the $100 \mathrm{~N}$ ink, Figure 
4.5 (a) and (b). In the case of the 50N50M ink, a low number of pulses results in a larger under sintering penalty, with a $2 \mathrm{~J} / \mathrm{cm}^{2}$ change in energy leading to a factor of 15 change in resistance Figure 4.5 (c). The impact of change in energy appears to be less dramatic for an increased number of pulses. Figure 4.5(d).

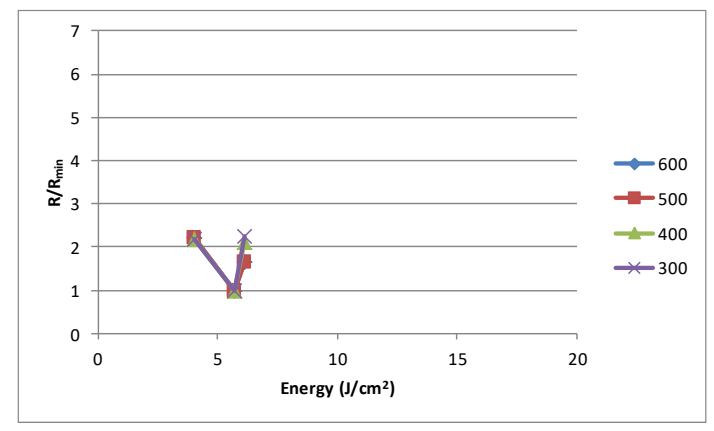

(a) $100 \mathrm{~N}-3$ Pulses

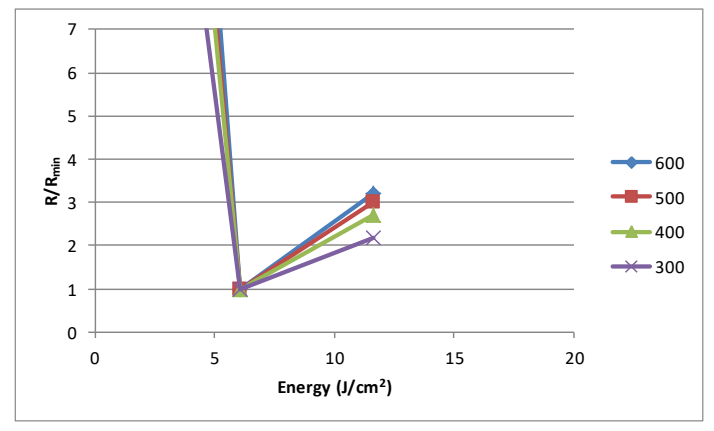

(c) $50 \mathrm{~N} 50 \mathrm{M}-3$ Pulses

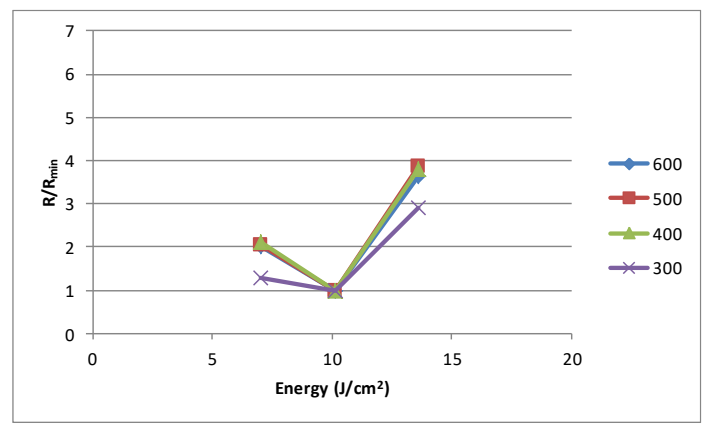

(b) $100 \mathrm{~N}-10$ Pulses

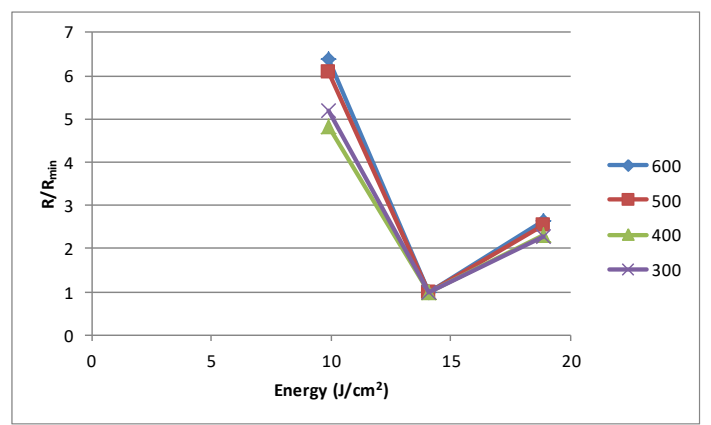

(d) $50 \mathrm{~N} 50 \mathrm{M}-10$ pulses

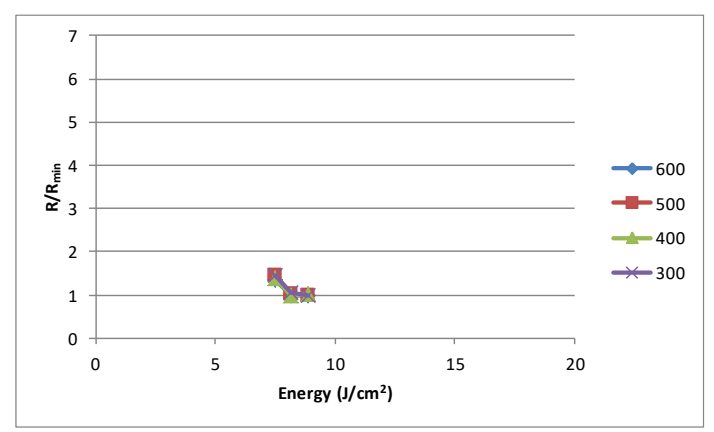

(e) $20 \mathrm{~N} 80 \mathrm{M}-10$ pulses

Figure 4. 5 Normalised resistance as a function of energy for each ink and line width. 
With the $100 \mathrm{~N}$ ink, there is some evidence that the smaller features are adversely affected by excess power, Figure 4.5 (a) \& (b), with the deviation from the optimum $\mathrm{R} / \mathrm{R}_{\min }$ being higher at the same power level for the finer structures.

The absolute line resistivity (calculated from the line profile, Chapter (2), at the optimum sintering energy for the $300-600 \mu \mathrm{m}$ line width with film thickness between ( $8-12 \mu \mathrm{m}$ ) for all materials is summarized in Figure 4.6. There is no specific trend in the respect to the line width and therefore the degree of sintering is independent of the feature size over the range considered.

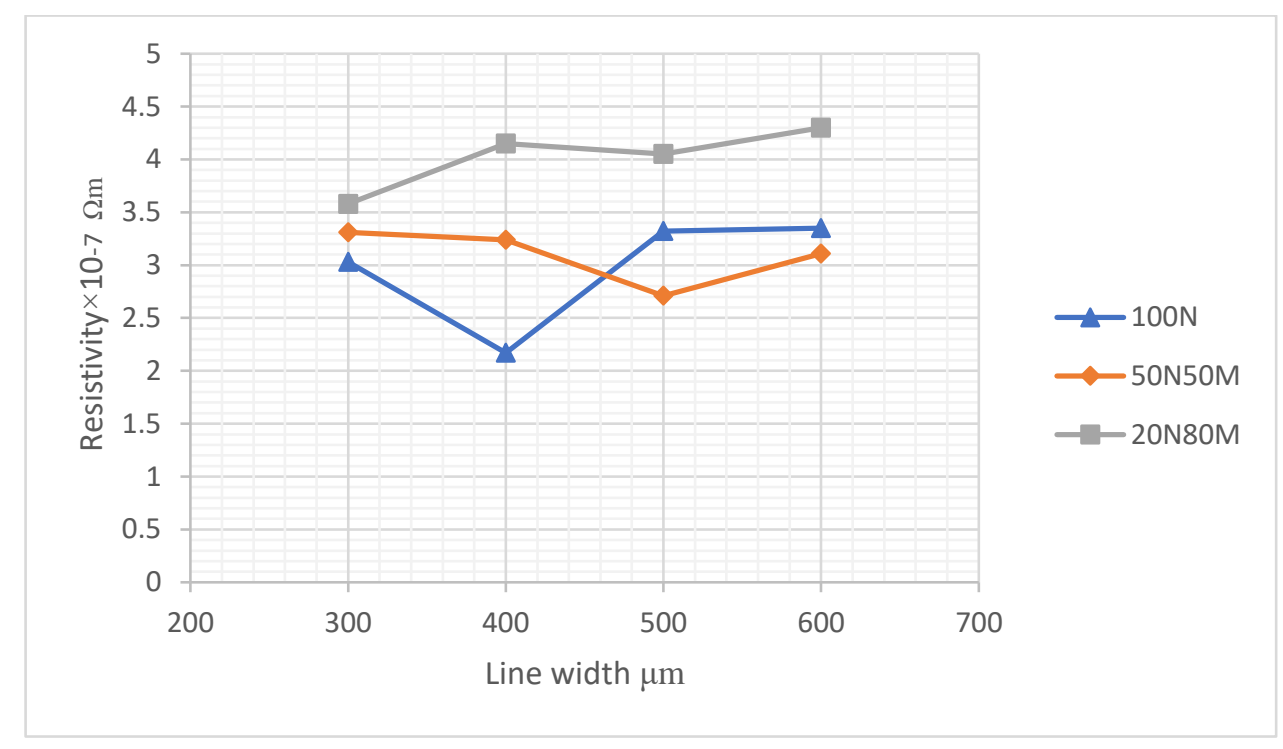

Figure 4. 6 Resistivity under photonic sintering condition

The absolute resistivity obtained with the photonic sintering was compared to the bulk copper resistivity and the best resistivity obtained with the thermal condition, Table 4.2. The measured absolute resistivity is between 13 times higher for the micro-nano blends inks than bulk copper and around 4-5 times higher than the thermal sintered the pure nano material ink. The resistance obtained under photonic conditions using $100 \mathrm{~N}$ ink is around 4 - 7 times that obtained with the reducing oven sintering for 600um line width, and approximately the same resistance for 50N50M and 20N80M ink. Photonic sintering is therefore as efficient as thermal sintering for inks containing micro based particles but does not reach the degree of sintering for inks containing only nanoparticles. 
Table 4. 2 Absolute resistivity relationship for each material for the 600um line (Photonic related to bulk and best obtained with thermal)

\begin{tabular}{|l|c|c|c|}
\hline \multicolumn{1}{|c|}{ Ink } & $\begin{array}{c}\rho \text { (abs Resistivity) } \\
* 10^{-7} \Omega \mathrm{m}\end{array}$ & Related to Bulk copper $\rho$ & Related to the best thermal $\rho$ \\
\hline $100 \mathrm{~N}$ & 3.35 & $10-12$ & $4-7$ \\
\hline $50 \mathrm{~N} 50 \mathrm{M}$ & 3.11 & 12 & 1 \\
\hline $20 \mathrm{~N} 80 \mathrm{M}$ & 4.3 & 13 & 1 \\
\hline
\end{tabular}

The optimum sintering settings for the $600 \mu \mathrm{m}$ line width for all materials is summarized in Table 4.3. There are no clear trends on which pulsing regime is used Applying a 10\% variation to the resistance which could be tolerated, then it is possible to estimate the process control requirements which would need to be imposed during photonic sintering. The inks containing nano materials are more sensitive to over sintering, resulting in catastrophic failure with small differences above the target. A wider range of intensities lower than the target are possible with the nano materials. The $20 \mathrm{~N} 80 \mathrm{M}$ ink is relatively robust to over exposure but can tolerate less under exposure as the larger particles may require higher energies to achieve good sintering and conductivity.

Table 4. 3 Optimum sintering conditions for each material for the 600um line

\begin{tabular}{|c|c|c|c|}
\hline Ink & $100 \mathrm{~N}$ & $50 \mathrm{~N} 50 \mathrm{M}$ & $20 \mathrm{~N} 80 \mathrm{M}$ \\
\hline Minimum R $(600 \mu \mathrm{m}) \Omega$ & 0.39 & 1.26 & 1.71 \\
\hline $\mathrm{R}_{\text {Photonic }} / \mathrm{R}_{\text {Thermal } \Omega}$ & 4.5 & 1.05 & 1.05 \\
\hline Energy Opt $\left(\mathrm{J} / \mathrm{cm}^{2}\right)$ & 10.14 & 6.09 & 8.88 \\
\hline Number of Pulses & 10 & 3 & 10 \\
\hline $\begin{array}{c}\text { Energy range }+/-\left(\mathrm{J} / \mathrm{cm}^{2}\right) \text { for } \\
10 \% \text { variation from target }\end{array}$ & +16 & -20 & +7 \\
\hline
\end{tabular}




\section{4: TLM measurement of Photonically sintered samples}

The trends observed in sheet and contact resistance in the thermal sintering section are generally repeated with the photonically sintered samples Figure 4.7, with typical contact resistances of between $1.1 \Omega$ and $5.9 \Omega$. The similarly in contact resistance $100 \mathrm{~N}$ and 50N50M ink resulted according to the energy applied which generally provided the lowest contact resistance at the sintering energy and higher contact with resistance with the high and low sintering energy applied, the $100 \mathrm{~N}$ and 50N50M have been omitted as poor film adhesion occurred and that attributed to the fast sintering which lead to a thermal shock which reduced contact area interface.

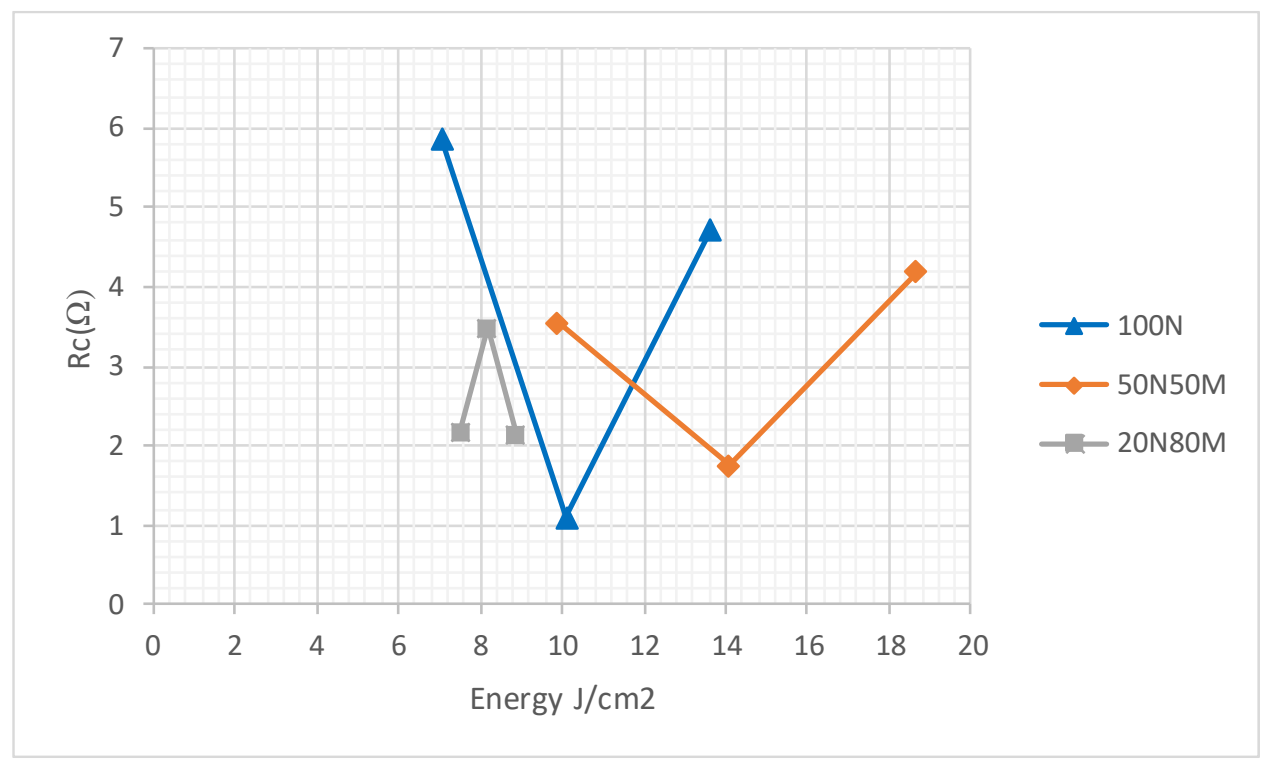

Figure 4. 7 Contact resistsiance for the photonically sintered samples.

Contact resistances for the micro / nano-particle inks are around twice that observed with the $100 \mathrm{~N}$, Figure 4.7 . This is likely to be due to the reduced contact area interface associated with larger particles.

\section{5: Sheet resistance by 4-point probe photonically sintered samples}

For the lowest line resistances in terms of optimum number pulses, the sheet resistance obtained by employing a four-terminal sensing measurement (RM3000) is displayed in Figure 4.8. The lowest sheet resistance achieved $(0.12 \Omega / \square)$ at $10.12 \mathrm{~J} / \mathrm{cm}^{2}$ with ink $100 \mathrm{~N}$ and at a point corresponding to minimum line resistance. For the 50N50M ink, the sheet resistance is opposite to the contact resistance results, the sintering energy applied $\left(14.09 \mathrm{~J} / \mathrm{cm}^{2}\right)$ resulted in a higher sheet resistance compared with other energy 
settings. The lowest sheet resistance achieved with $20 \mathrm{~N} 80 \mathrm{M}$ ink was at $8.88 \mathrm{~J} / \mathrm{cm}^{2}$ $(0.27(\Omega / \square)$.

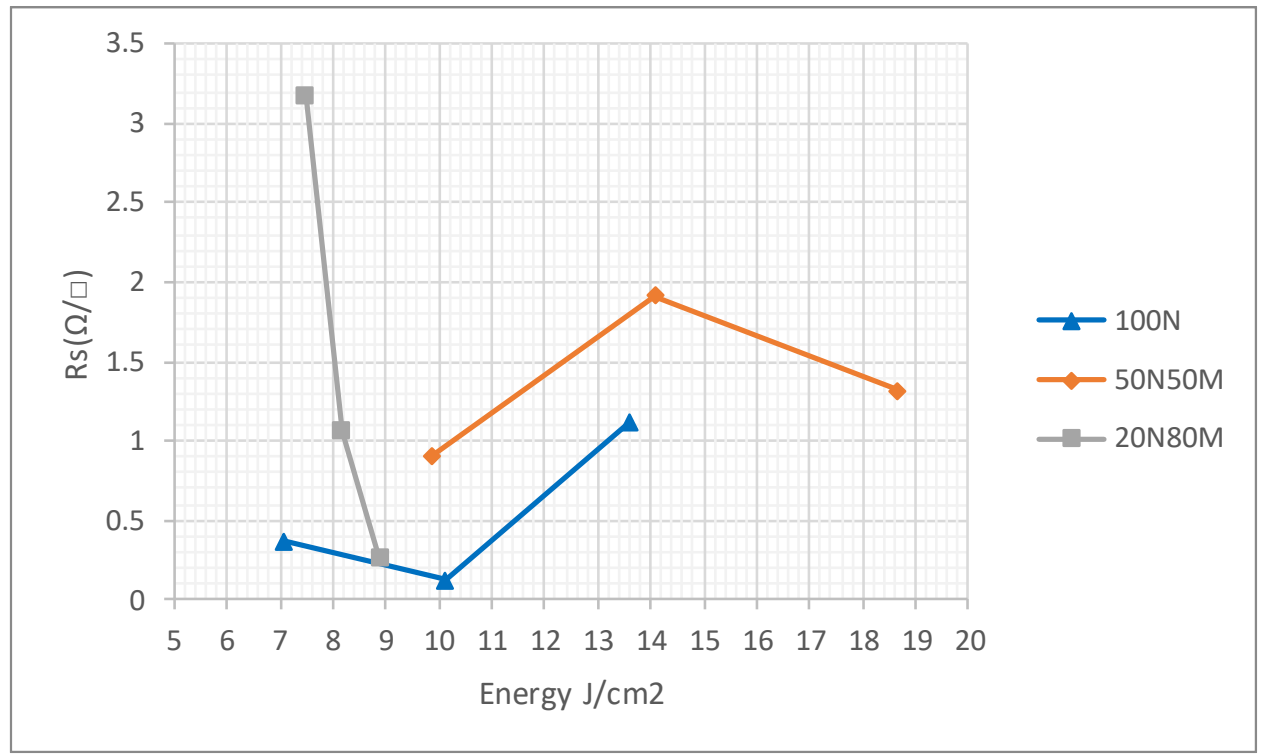

Figure 4. 8 100N, 50N50M and 20N80M ink, sheet resistance photonically sintered with different energy setting

\section{6: Adhesion test}

The adhesion test provides an interesting insight into the impact of the multi-pulse sintering and the interactions that occur between the substrate surface and the sintering ink. The $100 \mathrm{~N}$ ink in all cases showed $0 \mathrm{~B}$ adhesion level after the test, Table 4.4, indicating very low levels of adhesion. A possible explanation is that the multi-pulse sintering causes a volumetric reduction to the particle size [79], [156]. There may be rapid evaporation of the binder and the inter nano-particle sintering which induces a compressive stress in the film which overcomes the ink / substrate adhesion.

Table 4. 4 Adhesion tape test result

\begin{tabular}{|l|c|c|c|}
\hline Ink (nano-micro) & Sintering energy $\left(\mathrm{J} / \mathrm{cm}^{2}\right)$ & 0B-5B & Tape after test \\
\hline $100 \mathrm{~N}$ & 10.13 & $0 \mathrm{~B}$ & \\
& & & \\
\hline $50 \mathrm{~N} 50 \mathrm{M}$ & 6.09 & $1-3 \mathrm{~B}$ & \\
& & & \\
\hline $20 \mathrm{~N} 80 \mathrm{M}$ & 8.88 & $5 \mathrm{~B}$ & \\
& & & \\
\hline
\end{tabular}


The nano-micro -ink films had higher resistivity and a better level of adhesion strength than the pure nano ink. The lack of sintering and volumetric strain in these instances ensures excellent adhesion to the substrate. The 50N50M ink is removed in patches by the tape, which suggests that the micro-particles have an impact on the cohesion and adhesion of the printed film. But that the presence of the micro particles has improved structural coherence of the film and film/substrate interface. For 20N80M ink, the adhesion is such that the film leaves the surface only those areas where the film has been scored.

\section{7: Stability test after photonic sintering}

In a similar manner to the thermally sintered samples, the resistance change was measured over time in weeks for the three materials photonically sintered, Figure 4.9. There is a slight rise in resistance over the first week with the $100 \mathrm{~N}$ ink, followed by smaller increments over subsequent weeks. This is similar to the behaviour observed for thermally sintered material. The 50N50M also exhibited a rise in resistance, but the change more gradual and plateaued after 2 weeks. The 20N80M material exhibits minimal resistance increase over the period of measurement, inferring a very stable printed film. These trends would tend to indicate that the presence of a certain ratio of nano materials, particularly if not fully sintered, will increase the degradation of the film.

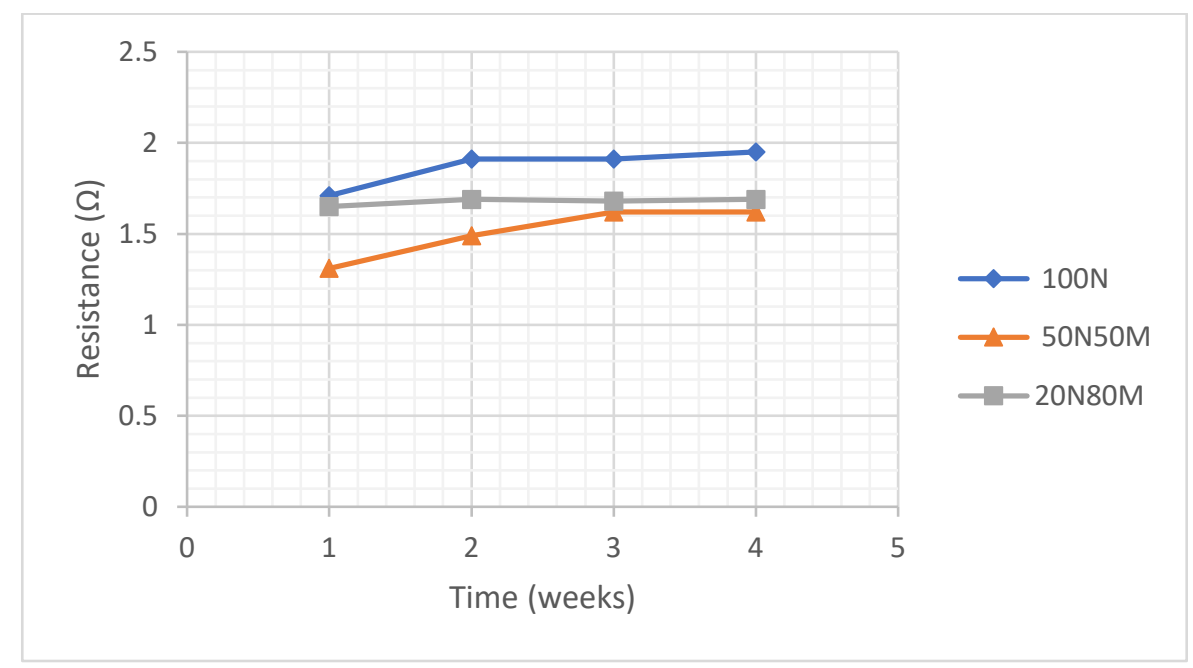

Figure 4. 9 Line resistance change over time in weeks for the three materials sintered 


\section{8: SEM photonically sintered materials}

The degree of sintering between the particles is considerably lower than the oven sintering. In order to better understand the mechanisms by which the printed film had been altered by the exposure to the pulsed light, selected samples were examined using SEM. Time constraints limited the number of samples which could be examined and thus the samples were selected based on the changes observed in the macro conductive electrical behaviour. Macroscopically, the photonically sintered films and the thermally sintered film (in this instance the film sintered in a formic acid environment) produced by the $100 \mathrm{~N}$ ink are similar, Figure 4.10 (a) and (b). Closer inspection at a higher magnification shows that in contrast to the thermally sintered films, the nanoparticles have not joined at the particle boundaries and there is only a small amount of evidence that the photonic sintering has melted the nano-particle boundaries, Figure 4.10 (c) and (d).. The increased porosity / lack of inter particle contact explains the reduction in conductivity seen with the $100 \mathrm{~N}$ ink compared to the thermally sintered materials, Table 4.2 .

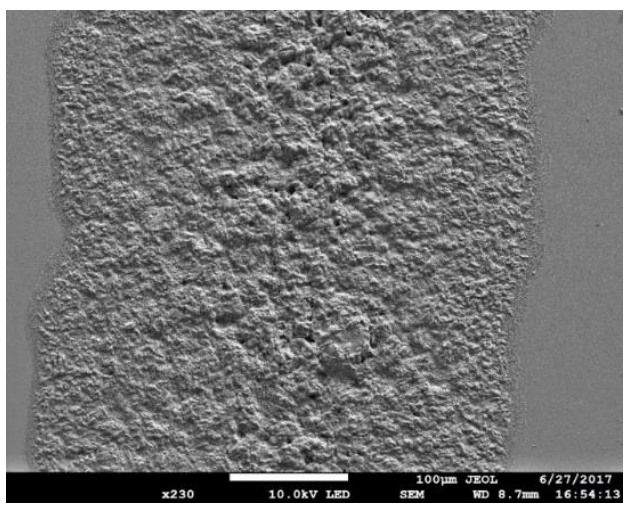

(a)

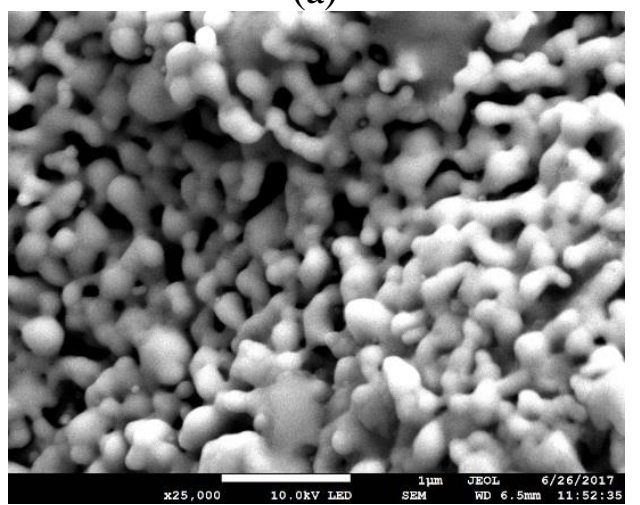

(c)

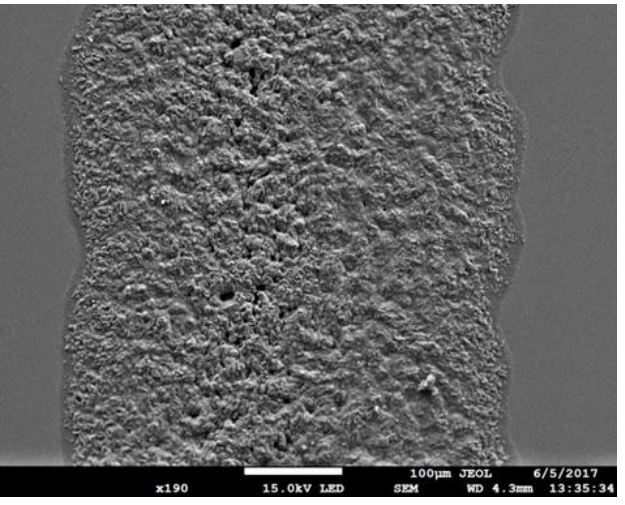

(b)

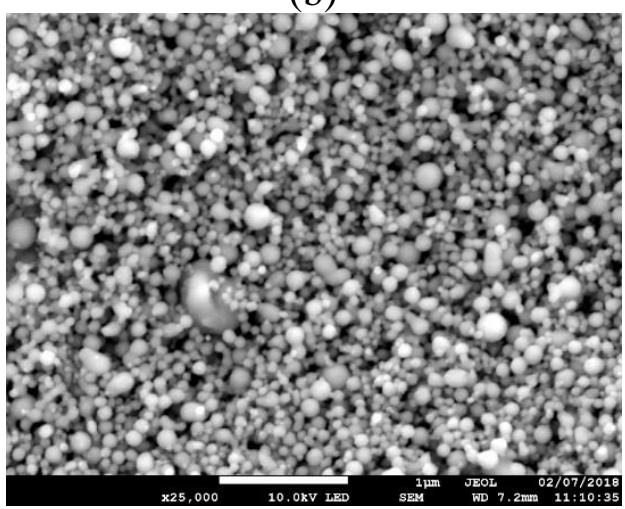

(d)

(a) Thermal sintered

(b) Photonic

Figure 4. 10 SEM images of the $100 \mathrm{~N}$ ink ( $\mathrm{a}$ and $\mathrm{b}$ ) thermally sintered (formic acid) and (c and d) photonically sintered films 
The poor adhesion for the $100 \mathrm{~N}$ ink is clearly illustrated in Figure 4.11 where a clear gap is observed between the nano-particle film and the substrate. As the adhesion of the dry film, but un-sintered film, was deemed reasonable then the act of photonic sintering has directly led to this delamination. A possible explanation is that the rapid sintering process causes a thermal shock on the interface and due to the relative coefficient of thermal expansion $\left(9 \times 10^{-6} /{ }^{\circ} \mathrm{C}\right.$ for glass compared to $17 \times 10^{-6} /{ }^{\circ} \mathrm{C}$ for bulk copper) a stress is induced at the interface. There is also the possibility of the temperature required for bonding (via a sintering process) has not been achieved but the impact of the photonic sintering is to eliminate the binder locally from interface.

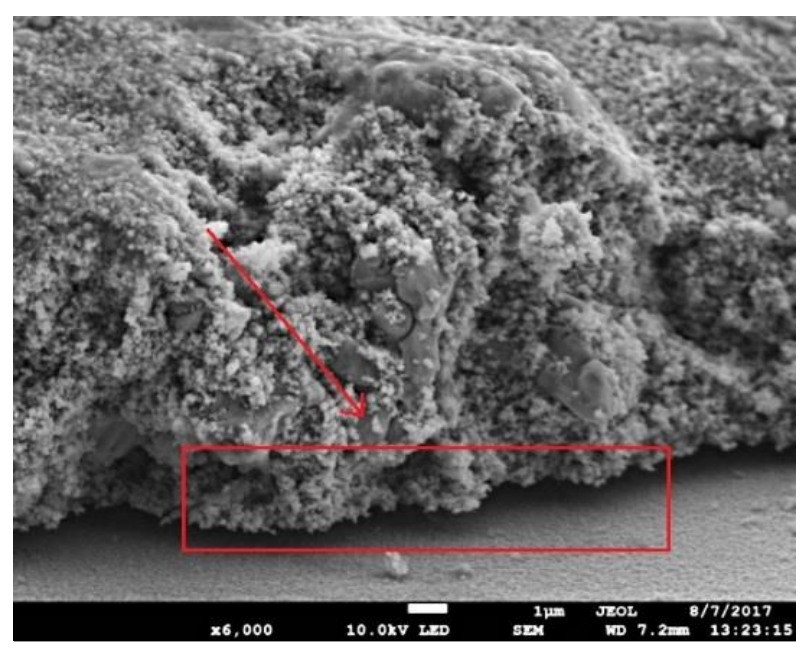

Figure 4. 11 FTO - $100 \mathrm{~N}$ Interface when photonically sintered

There is some evidence of extensive pinholes in the 50N50M ink film when examined macroscopically, Figure 4.12 (a) - (d). It is hypothesized that these could be associated with "solvent boil", a phenomenon where any residual solvent in the film is converted into the gas phase rapidly, creating a void in the film from the bottom to the surface. Similar structures are observed on a micro-scale where the nano-particles remain as discrete spheres on the surface of the micro particles. As with the thermal sintering, the presence of the micro particles is an obstacle for melting of the nano-particles. The similarity in the structures is reflected in the similarity in the electrical performance of the material, Table 4.2 . 


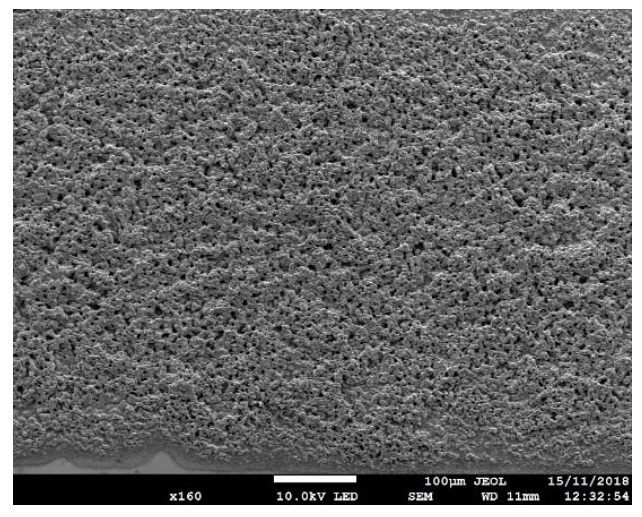

(a) Thermal

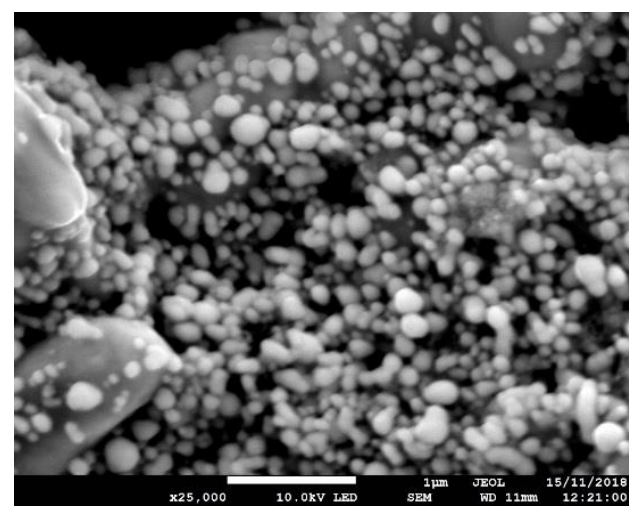

(c) Thermal

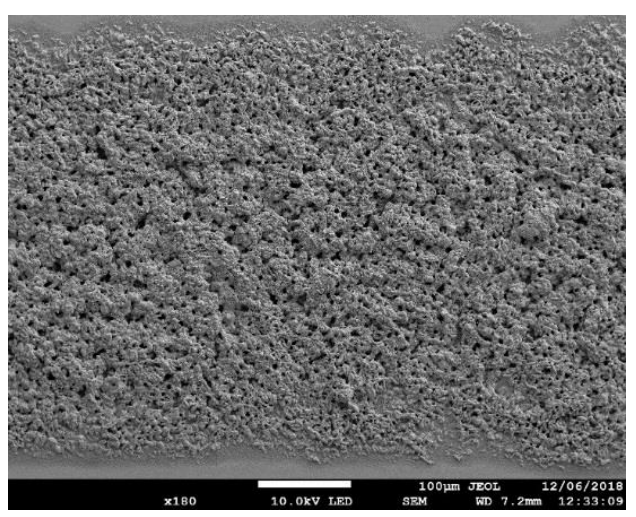

(b) Photonic

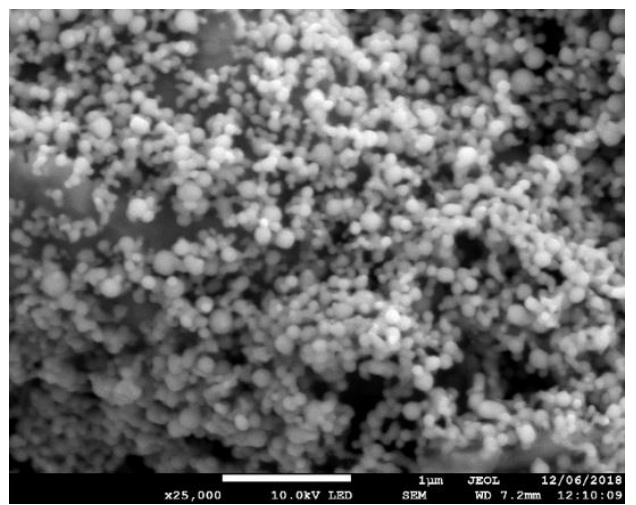

(d) Photonic

Figure 4. 12 SEM images of the thermally and photonically sintered films for the $50 \mathrm{~N} 50 \mathrm{M}$ ink.

The microstructure of the photonically sintered $20 \mathrm{~N} 80 \mathrm{M}$ is similar to the thermally sintered sample, Figure 4.13 (a) - (d). There is again evidence of small voids in the surface which may be associated with solvent boil as described earlier. Closer inspection shows further similarity with the nano-particles observed as discrete spherical like objects which are bound to the micro particles, but which have not melted to coat the surface of the micro particles. As with the 50N50M ink, the similarity in the microstructure has resulted in similar electrical performance. 


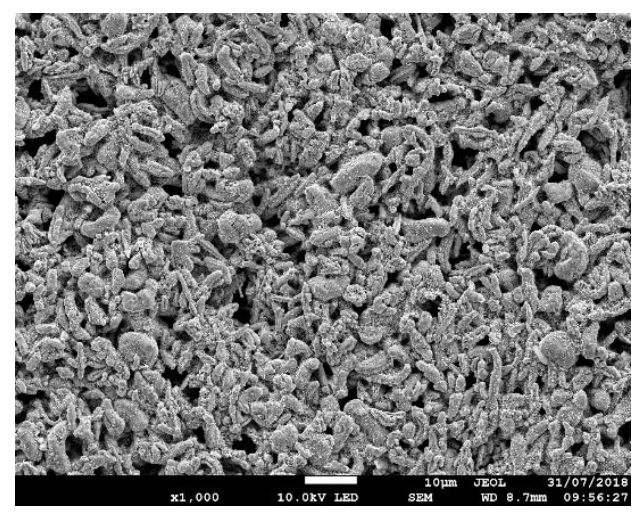

(a) Thermal sintered

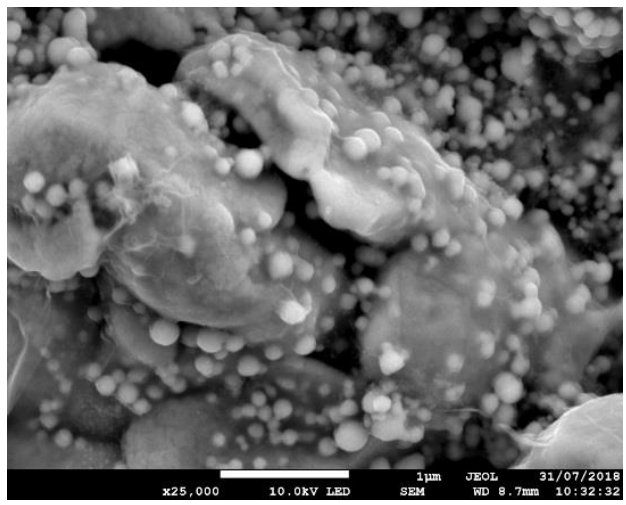

(c) Thermal sintered

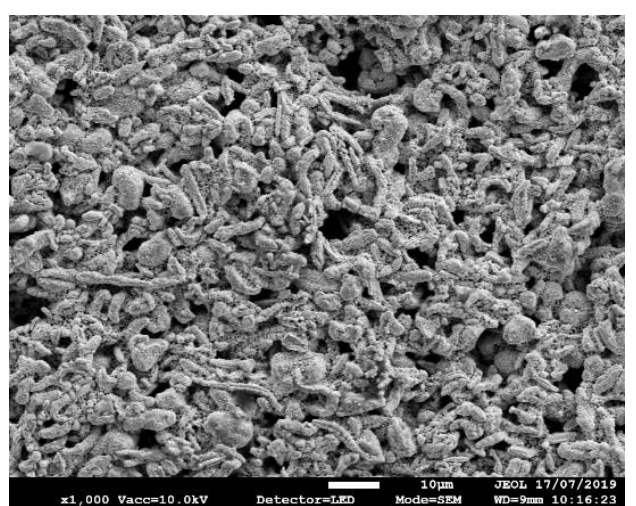

(b) Photonic

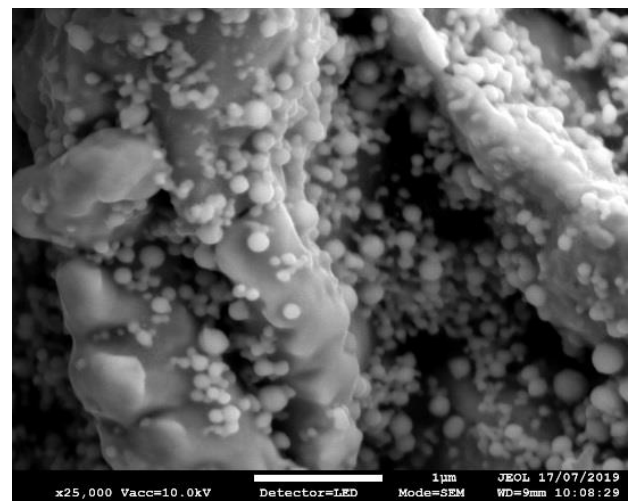

(d) Photonic

Figure 4. 13 SEM images of the Formic and photonically sintered films for the 50N50M ink.

\subsection{X-ray diffraction (XRD) Photonic sintering:}

$\mathrm{X}$-ray diffraction of the examined printed copper film using $100 \mathrm{~N}$ ink shows that diffraction peaks with strong intensities appear at angles corresponding $2 \Theta 111,200$ and 220, this means the copper is formed and stable against oxidation with all energy settings applied [157]. The 50N50M ink also shows that diffraction peaks with a weaker intensities appear at angles corresponding $2 \Theta 111,200$ and 220 with the high energy applied $\left(14.09,18.66 \mathrm{~J} / \mathrm{cm}^{2}\right)$, but with $9.89 \mathrm{~J} / \mathrm{cm}^{2}$ intensities appear at angles corresponding $2 \Theta 110,111,200$ and 220 , this means the copper is formed with less stability against oxidation (The XRD results of the 100Nand 50N50M ink prepared and displayed in Figure 4.14. 


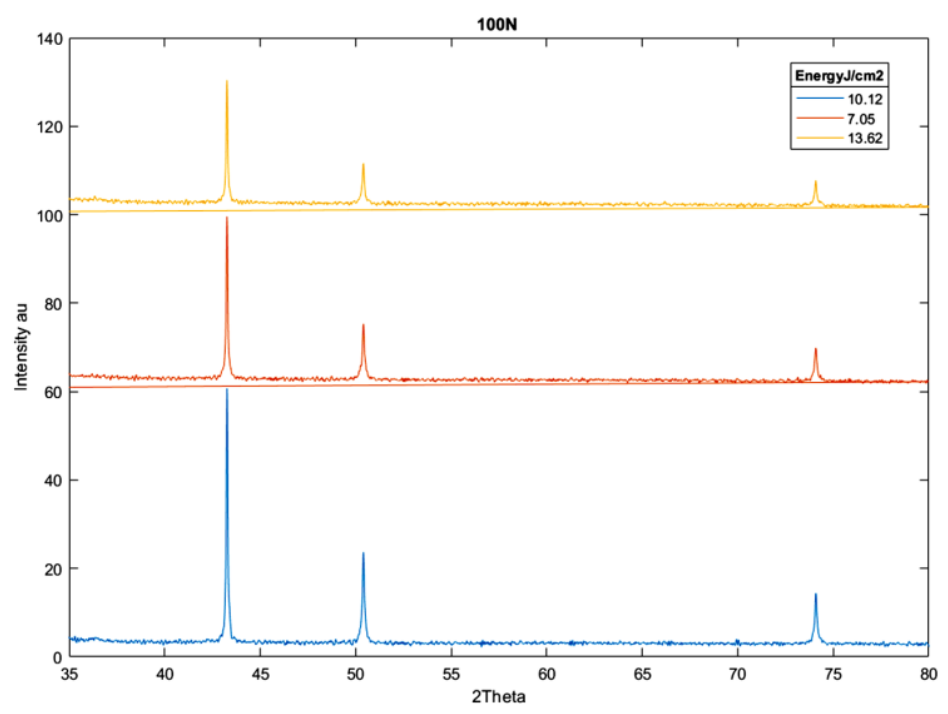

(a) $100 \mathrm{~N}$ ink

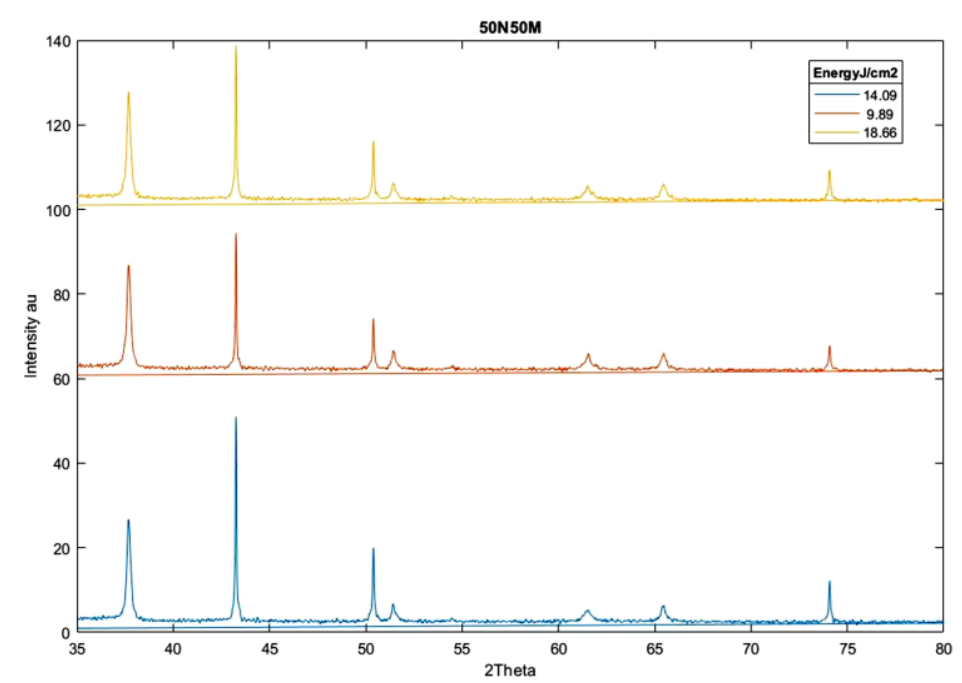

(b) $50 \mathrm{~N} 50 \mathrm{M}$ ink

Figure 4. 14 XRD of the 100N, 50N50M ink -copper patterns (600 $\mu \mathrm{m}$ line width)

A limited oxide formed during photonic sintering of 20N80M ink is evident in Figure 4.15, where the relative copper intensities strength for the photonically sintered samples is on par with the all energy applied. For reference the samples sintered with $8.17 \mathrm{~J} / \mathrm{cm}^{2}$ energy applied shows the show a significant decrease in $\mathrm{CuO}$ peak. 


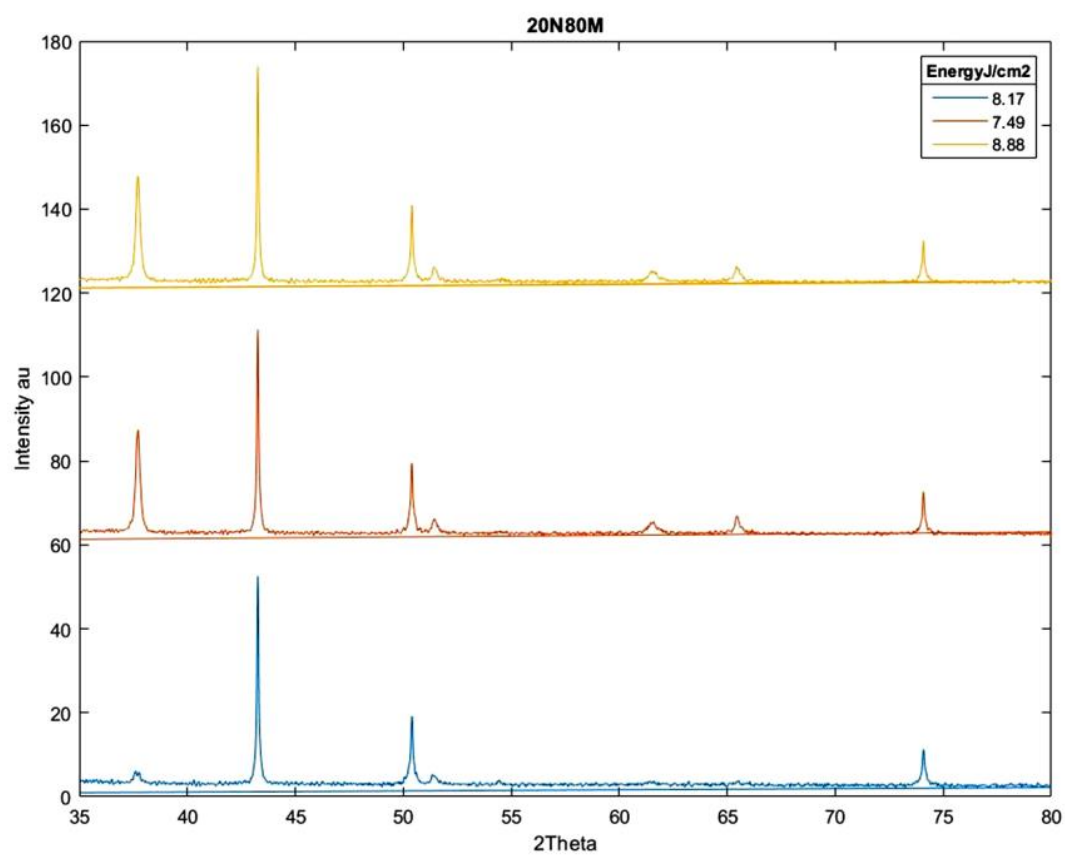

Figure 4. 15 XRD 20N80M ink (600 $\mu \mathrm{m}$ line width)

The limited quantity of oxide formed with 20N80M ink also illustrated in Figure 4.16 with comparison to the $100 \mathrm{~N}$ where were no oxide formed. The relative copper intensities strength for the photonically sintered samples is showing that the $100 \mathrm{~N}$ ink (nano-particles 100\%) provided the highest copper peak [10].

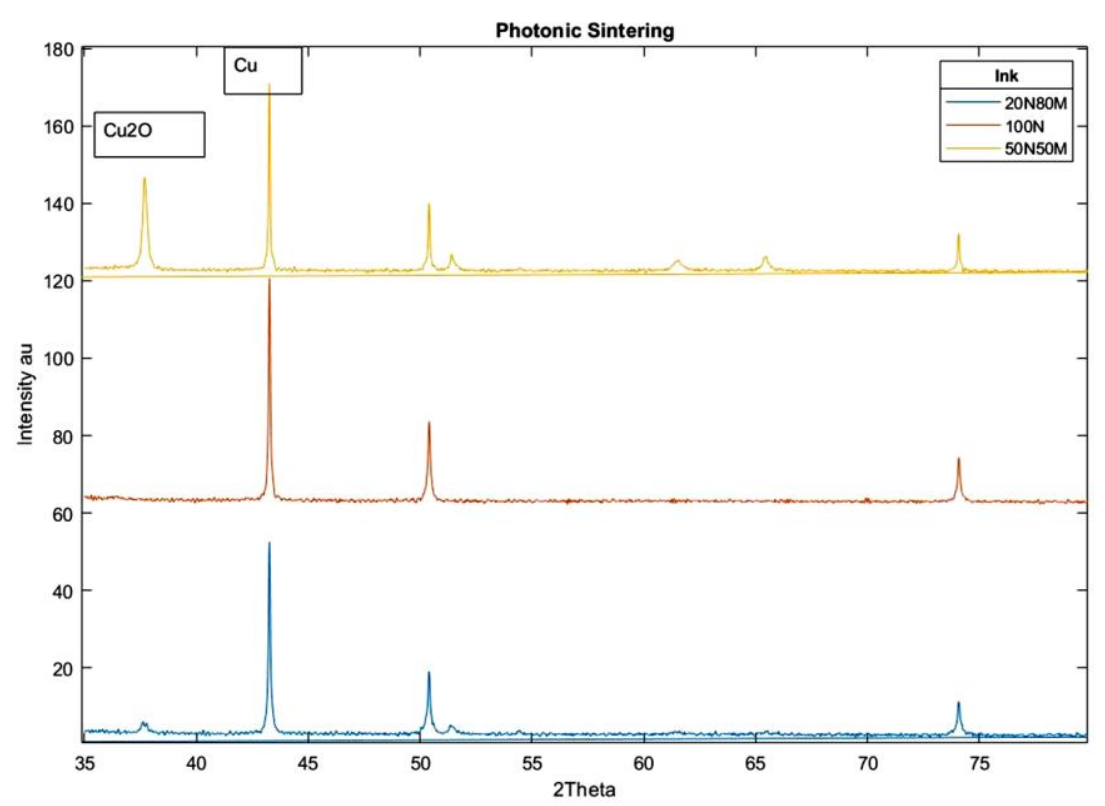

Figure 4. 16 XRD 100N,50N50M and 20N80M ink (600 $\mu \mathrm{m}$ line width) 
The XRD spectra for $100 \mathrm{~N}$ ink provide similar spectra to the ink $20 \mathrm{~N} 80 \mathrm{M}$ which suggests that the $\mathrm{Cu}_{2} \mathrm{O}$ which is absence in the photonic sintered sample does not undergo significant reduction with ink 50N50M under photonic sintering process.

\subsection{0: Discussion}

This study has shown that it is possible to photonically sinter nano-copper thick screen-printed films. The degree of success of the sintering is dependent on material and operational factors and has implications for

\section{Operational viability}

2. Underlying Mechanisms

\subsubsection{Operational viability}

For a purely nano ink, the performance of the photonically sintered features reduced significantly from the thermally sintered materials. The relative conductivity and the loss in adhesion do not make photonic sintering a viable option for manufacturing.

With the micro-particle inks, adhesion is excellent, and performance is equal to that achieved with the thermal sintering. Photonic sintering, therefore, provides a viable alternative to thermal sintering for this material, although at the expense of the absolute conductivity. From an industrial perspective, the photonic sintering process looks promising, particularly if considering the use of microparticle inks as the sintering process takes the order of seconds compared to many hours in a thermal sintered process. Besides, no controlled gas environments are required, and thus safety issues and operating costs are reduced. Photonic sintering could be used as part of a continuous process, instead of a batch process which has significant advantages in terms of productivity, scheduling and work in progress, which would have a positive impact on the operational costs. The primary challenges faced with implementing photonic sintering are associated with exposure area, the link between ideal exposure and feature size and establishing the manufacturing tolerances. The $50 \times 50 \mathrm{~mm}$ sample was placed in the centre of the $200 \times 250 \mathrm{~mm}$ exposure bed in order to limit any variation. Further work would be required to examine whether variation that might 
be experienced under a larger area of illumination, would have an impact on the sintering performance. Establishing the tolerances for the lamp exposure would require finer refinement of the energy \& pulse length around the optimum values identified. These would need to be monitored as the lamp output could vary under continuous operation and as the lamp ages, [155].

A clear link was seen between the degree of sintering and the feature size with the large solid areas being characterized by sintered edges (as viewed by colour) and an un-sintered centre region Figure 4.4 (c). This has design implications as all elements within the "circuit" would need to be within a dimensional range in order for photonic sintering to be successful, those above the limit would not be fully sintered, while those below the dimensional range would be catastrophically affected, Figure 4.4. Such design constraints could have a significant impact on photonic sintering viability. For example, in the design of PV module narrower features (40-50 $\mu \mathrm{m})$ are required to locally collect charges from the open active areas where charges are generated [158]. These in turn are collected to larger bus bars which are used to provide a low resistance pathway for the charge away from the cell. Photonic sintering may not therefore be a plausible method for this application where the design fundamentally requires a mixture of optimized sintering conditions.

\subsubsection{Underlying Mechanisms}

The lack of sintering (Figure 4.10) observed with the 100N ink suggests that either the peak temperature within the film is not reached or that the peak temperature is not held for a sufficient length of time for the sintering process to occur. Given that sintering times for nano-copper [17] [16] and nano-silver [100] [113] have been shown to be less than $10 \mathrm{~ms}$ using laser sintering, the most likely explanation is that the sintering temperature is not achieved.

There are a number of suggestions that the FTO plays a role in the sintering of the film. The sintering seen around the edge of larger areas would suggest that there is a temperature difference between the outer edges and middle of the film. The transmission of Tec 7 FTO coated glass is around 82\% [159] and relatively even across the visible spectrum. Thus, in excess of $15 \%$ of the remaining light is likely to be 
absorbed and results in a temperature rise. This could result in a thermal shock which overcomes the sintering level occurred in the middle area.

The near equal performance of the micro / nano-particle blend ink and the similarity in the micro structure reinforces that the photonic sintering process is sufficient to remove the majority of the binder from the ink and create the bond to the surface without the need to sinter / melt the nano-particles.

The study has shown that there may be multiple energies which are capable of sintering the copper inks with near equal resistance being observed with similar energies. For example, the line resistance is similar at $6 \mathrm{~J} / \mathrm{cm}^{2}$ and $14 \mathrm{~J} / \mathrm{cm}^{2}$ for the $50 \mathrm{~N} 50 \mathrm{M}$ ink, Figure 4.3 (b), with the primary changes being the number of pulses and the absolute intensity of the pulses. There is clearly a dynamic element in the rate of energy absorption, the sintering process and any associated cooling which occurs between pulses. A direct instrumented experimental investigation of this process is difficult given the timescales involved, the extreme conditions experienced within the film during the exposure process. In addition, the destructive nature of the tests and the limited material quantities available did not allow a wider investigation of the complex interaction between the input parameters from the output properties of the sintering process.

Dynamic models for photonic sintering of other ink substrate combinations have been developed [80], but these are reliant on physical constants which are not readily available for the material (e.g. final and initial thermal conductivity), empirically derived constants (e.g. for cooling mechanism) and targeted towards thin films on thin substrates. Through film conduction and high thermal gradients within the film depth and the large heat sink associated with a thick piece of glass are likely to deviate the performance from these scenarios. It was initially hoped that a modelling approach could be used, but it was decided that number of unknown constants and the additional issues in addressing the through thick film condition and geometric feature considerations would have been of limited use. 


\subsection{Conclusion}

An experimental study of the photonic sintering of copper nano micro inks has been carried out. The primary conclusions of this study are:

1. It is possible to photonically sinter the screen-printed copper films on FTO glass substrates.

2. There is $5-7$ times reduction in conductivity for the pure nano-particle ink when photonically sintered and this can be associated with the lack of nano-particle sintering with in the film.

3. Equal electrical and adhesion properties can be obtained for the micro - nanoparticle blends. This offers a means of sintering without controlled gas environments within seconds, when compared to hours in thermal sintering.

4. There is a multiple optimum values of energy and this is reliant on the number of pulses which are used for the sintering

5. The operational window for sintering is small for the purely nano material and is wider for the nano / micro particle blends. Operation at energy levels below this window does not achieve sintering, while catastrophic failure occurs below this value. 6. The optimum sintering conditions vary with the feature size which limits the flexibility of the circuit design.

The next thesis chapter investigates other technologies which can be used to rapidly sinter thick film copper printed materials on FTO substrates which overcomes some of the issues highlighted in this chapter. 


\section{Chapter 5: Alternative fast sintering technique}

\subsection{Introduction}

This chapter presents the sintering results achieved by employing other fast sintering methods other than flashlight and thermal sintering which were introduced in chapter 3 and 4. The materials and the equipment employed in this chapter were the same as used in previous chapters. While wide area white light photonic sintering was successful, it produced challenges. It requires that the same sintering condition is applied to the whole surface, impacting the degree of sintering when feature dimensions change, and the operational window is limited with possible catastrophic failure when over sintered.

Laser sintering offers selective condition to each object printing, or with ability to apply a different condition for each print size on the circuit. Also, laser sintering would ignore any issues caused by the energy absorption by the FTO.

NIR (Near Infrared) can also be used as a fast sintering process. The main advantages of using the NIR are that it is more widely available than pulse light, it absorbs in a different part of the spectrum [160] and as absolute energy intensities are lower the operational window in terms of time may be larger and more manageable. Even with processing times in the order of 10s of seconds, this would still provide a productivity advantage over thermal sintering.

Through iterative studies with refinement of methods, the potential of these attractive processes will be examined.

\subsection{Optimization of Laser Sintering}

A Rofin power line $\mathrm{E}$ was the laser technique selected for these tasks because its light, as the $532 \mathrm{~nm}$ wavelength absorption rate range was deemed the best setting for the application. An initial study was carried out at each wavelength within selected values within a power range between $10 \mathrm{~W}-40 \mathrm{~W}$ and a frequency range of 10-200 kHz. This range was limited by the operational window of the laser system. The laser was set in a raster mode where it scanned across an area rectangle which included the printed lines and the background FTO. This was necessary as optical registration between the sample and the laser could not be guaranteed. 
As material samples were limited, the initial conditions were set according to discussions in the laboratory with users who utilize the same laser for laser machining FTO, sintering $\mathrm{TiO}_{2}$ and scribing glass for ease of cutting. From this initial starting point, the power, frequency and wavelength of the laser was varied in line with the observed macro change in the exposed regions and, where appropriate, resistance measurements.

The initial settings which were operated at an intermediate power level were found to cause catastrophic failure of the film with complete delamination. The power and frequency were subsequently reduced to its lowest level (10W and $10 \mathrm{kHz})$ which allowed the film to remain visible on the surface with a perceptible and significant colour change indicating a change in physical structure had occurred.

Table 5. 1 Laser settings used in the investigation at wavelength of 532nm

\begin{tabular}{|c|c|c|c|c|}
\hline Sample & Power & Frequency & Result observed & Image \\
\hline $100 \mathrm{~N}$ & $40 \mathrm{~W}$ & $200 \mathrm{kHz}$ & Damage & \\
\hline & & & & \\
\hline $50 \mathrm{~N} 50 \mathrm{M}$ & $40 \mathrm{~W}$ & $200 \mathrm{kHz}$ & Damage & \\
\hline 3 & & & & \\
\hline $20 \mathrm{~N} 80 \mathrm{M}$ & $40 \mathrm{~W}$ & $200 \mathrm{kHz}$ & Damage & \\
\hline
\end{tabular}




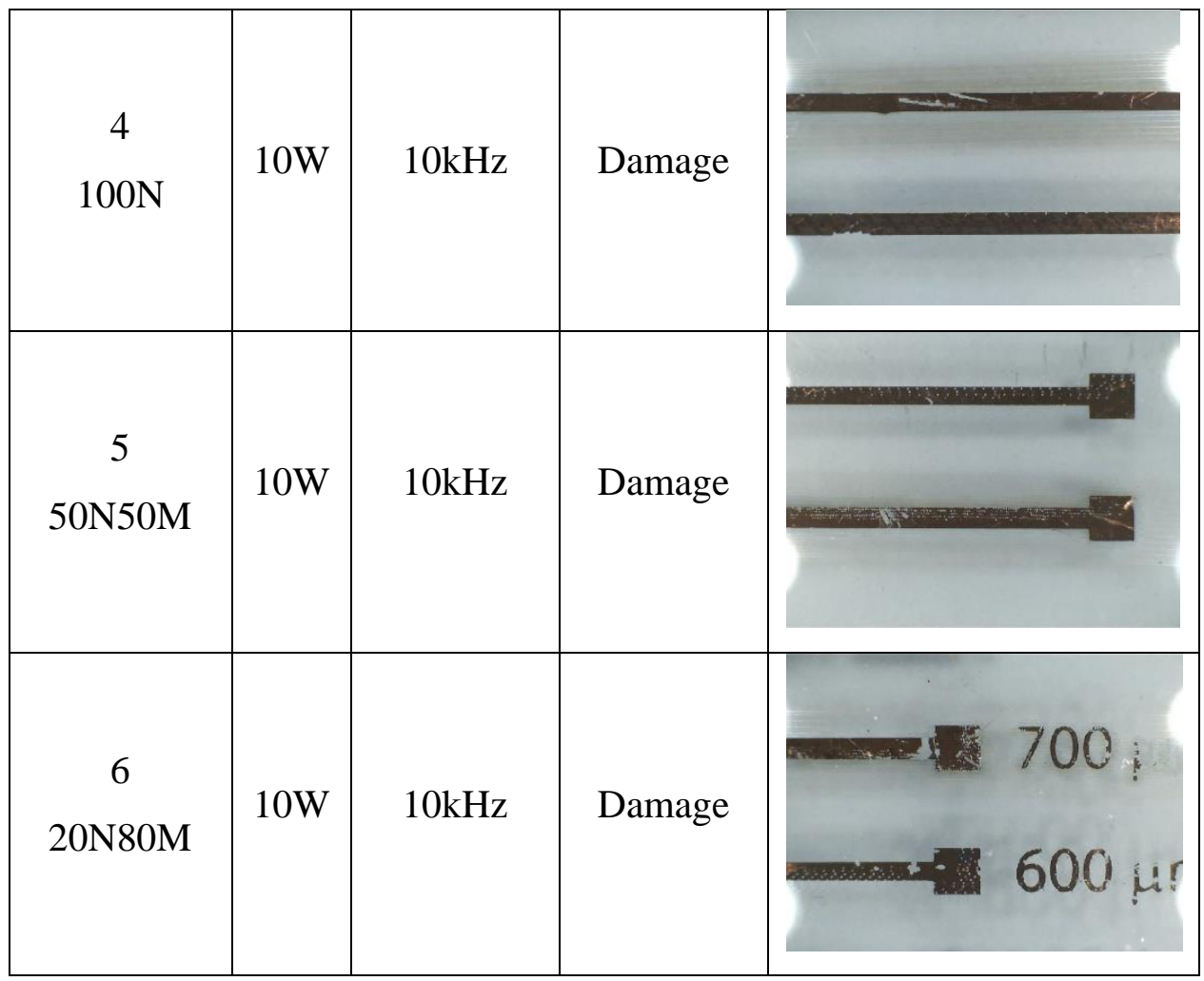

Although a visible change in each of the samples was observed, resistance measurements could not be reliably taken. In order to identify possible causes for this, samples which were produced at the lowest power level and lowest frequency were studied using SEM. Figure 5.1 shows the SEM images of the samples sintered with laser which were visibly observed as the best quality compared to photonically sintered samples. In each instance the microstructure and surface topography of the laser exposed surface differs considerably from that of the photonically sintered surface. The laser exposed surface for the $100 \mathrm{~N}$ ink is highly non uniform and characterized by surface cracks and localised divots in the surface, Figure 5.1 (a). There are also regions where some degree of localized sintering has occurred which seem randomly distributed across the area. The surface of the $50 \mathrm{~N} 50 \mathrm{M}$ is again highly non uniform with areas in the lower part of the image where melting between the nanoparticles has clearly occurred and areas where nano-particle sintering has not occurred. There is clear evidence of large cracks which cause chasms across the surface of the film. Such damage would clearly have a negative impact on conductivity. The 20N80M shows a significant amount of nano-particle and microparticle melting with the particles having a globular nature and areas where a continuous metal film has been formed. 


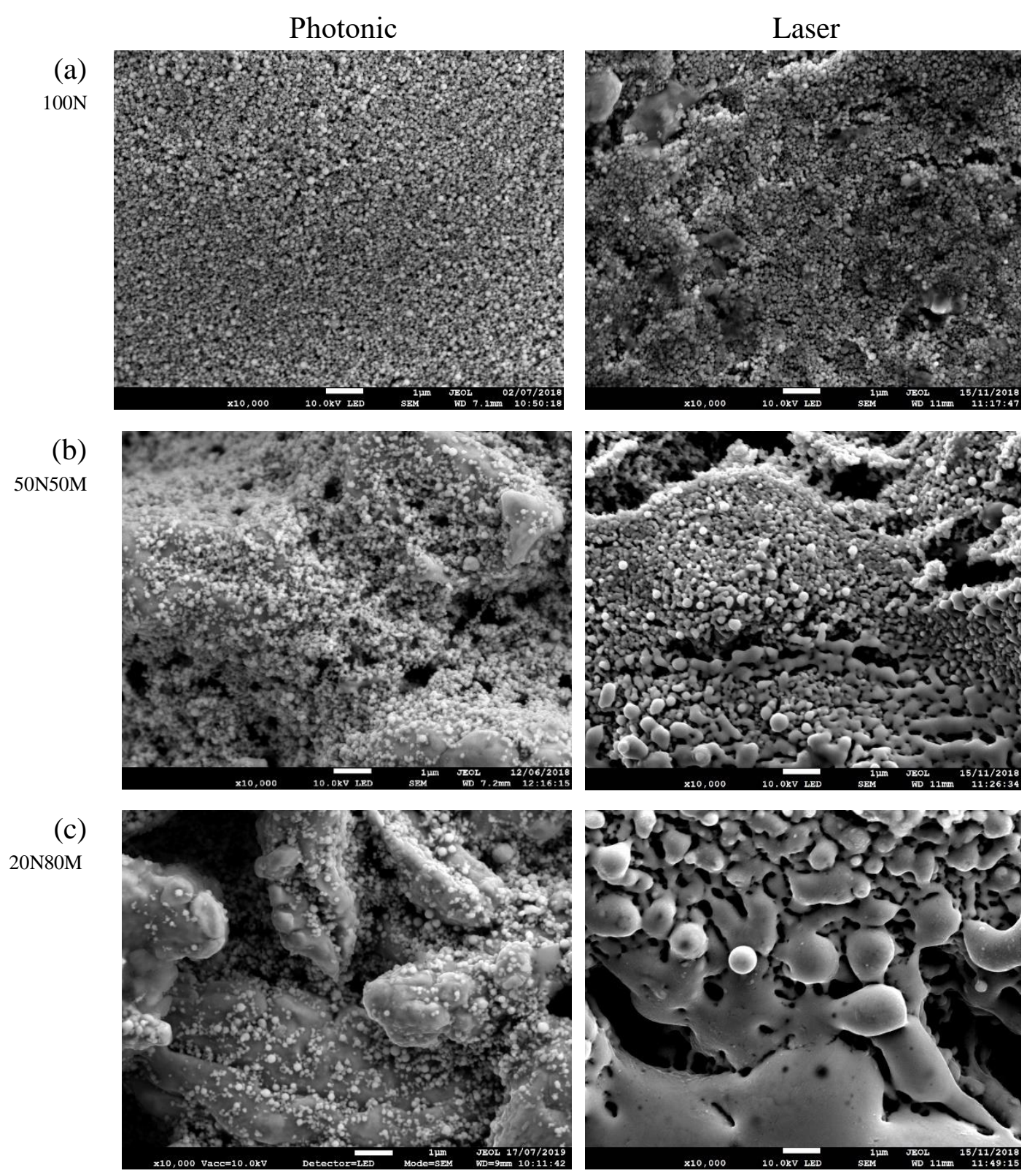

Figure 5. 1: SEM images of Photonic sintering vs laser sintering for laser samples 4,5 and 6.

The significant change and structure damage caused by the laser exposure is result of the localised nature of the laser exposure fluence and this has been seen previously in the literature,. [161], [162]. The localised nature of the laser sintering and short time scales is a rapid energy transform phenomenon that has led to damage and cracks in the copper film. It has been postulated that damage caused by the laser is a result of the anisotropic stresses and stress waves which can increase the dislocation density 
and roughness of the melting front for the printed film [163], which then opens particular heat conduction channels through the film. This concentrates the energy resulting in highly localised morphological changes. The laser exposure also caused delamination of the copper film in all instances. Accordingly, any adhesion tests were rendered invalid.

The laser wavelength, power and frequency used has been shown to be inappropriate for sintering thick screen-printed copper nano and micro inks. The overall fluence of the laser produces localized damage to film, even at its lowest setting. Ideally, alternative lasers would have been tested, but this was not possible given the number of samples sacrificed during this study and the availability of lower power lasers.

\section{3: Near infrared (NIR) sintering}

NIR offers a means of direct heating of the sample over a period of tens of seconds [164], [165], [166] offering the opportunity to lower energy densities which was shown to have such a negative impact of film integrity with laser and photonic sintering.

The copper ink was sintered by NIR irradiation at room temperature and ambient conditions. The NIR system used in this study used of 6 twin tube NIR lamps. The maximum electrical power density of the system was $250 \mathrm{kWm}^{-2}$ and a minimum sintering time of 20 seconds was set up because a stable time was needed for NIR light irradiation and the applied power and on-time were adjusted to change the sintering conditions. As there was no guiding literature available for sintering the copper, the initial conditions were chosen from conditions used by equipment owners who had experience of heat-treating glass and metal substrates. From these initial tests the power and time of exposure were steadily increased from $20 \%-100 \%\left(50 \mathrm{kWm}^{-2}\right)$ with variety of sintering time period $(20-60$ seconds $)$.

The perceived and macroscopic change in visual properties of the $20 \mathrm{~N} 80 \mathrm{M}$ copper ink sintered on the FTO glass are shown in Table 5.2. This NIR technique was found ineffective to sinter the copper ink used in this study, as low power between 20-60\% with sintering time (20-60 s) does not affect the electrical properties of the copper ink. 
Conversely, applying power over $60 \%$ leads to an over-sinter for all materials employed (100N, 50N50M and the 20N80M). Thus, the operational window in order to produce the optimum level of sintering was deemed to be small.

Table 5. 2: NIR power used in the investigation (20N80M ink)

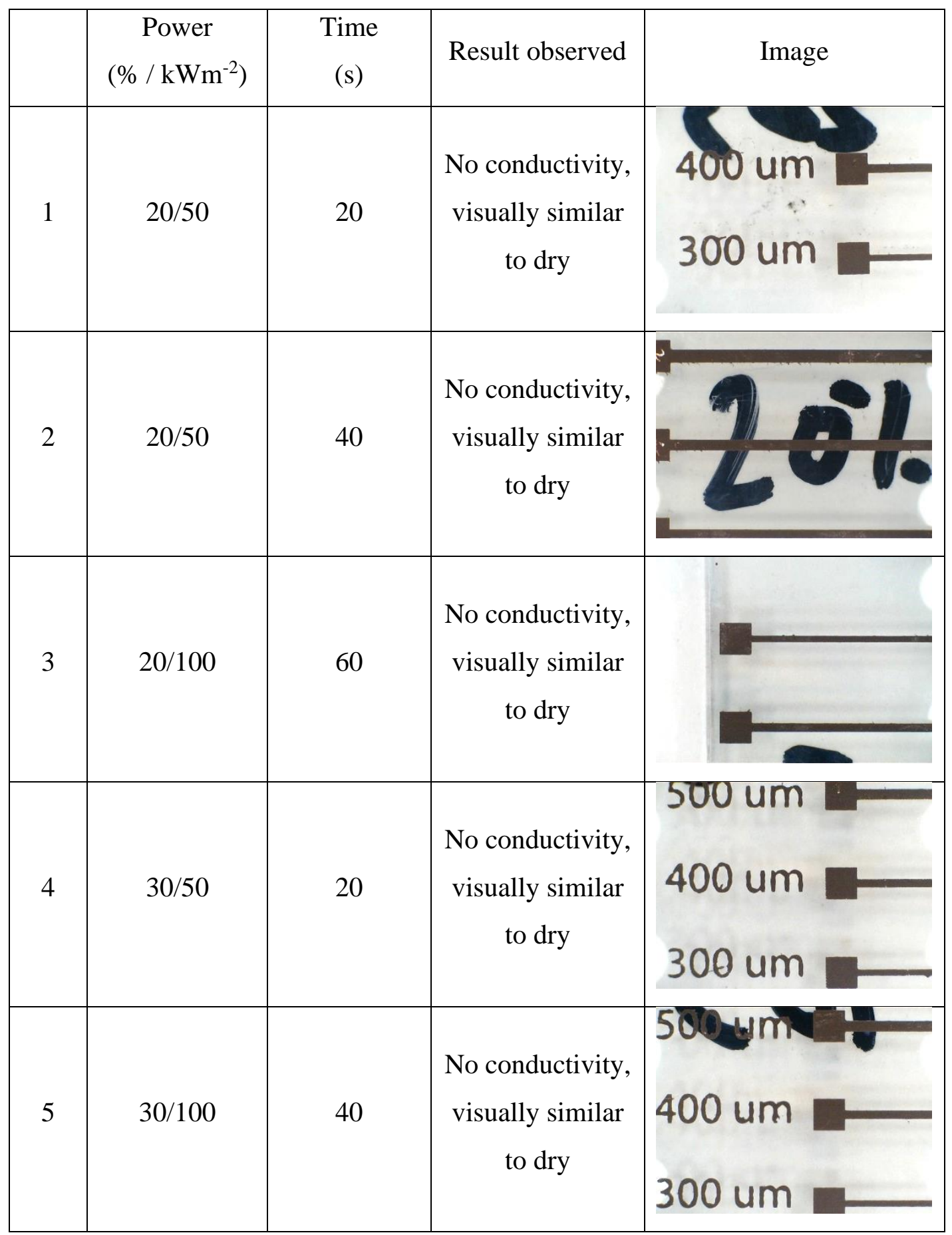




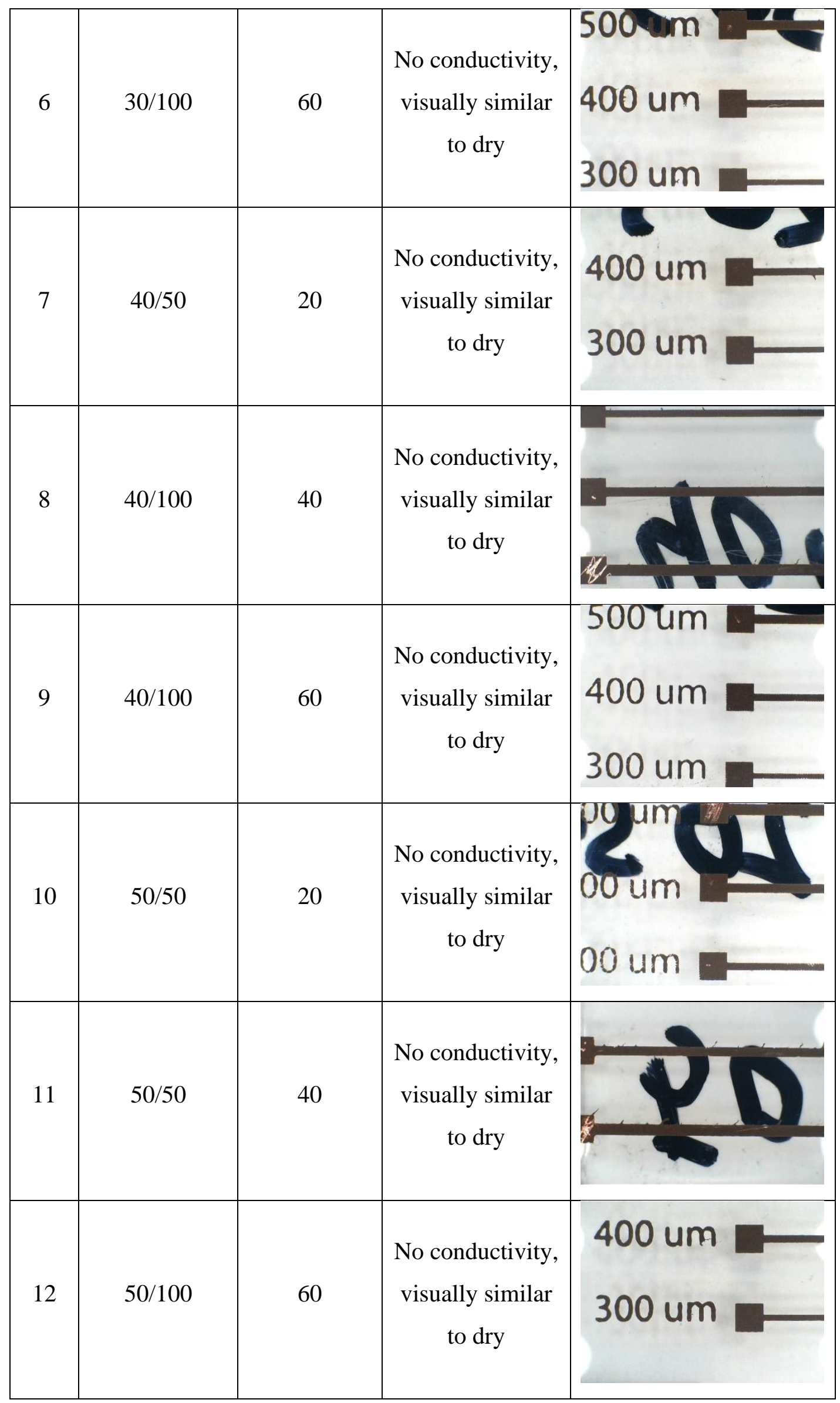




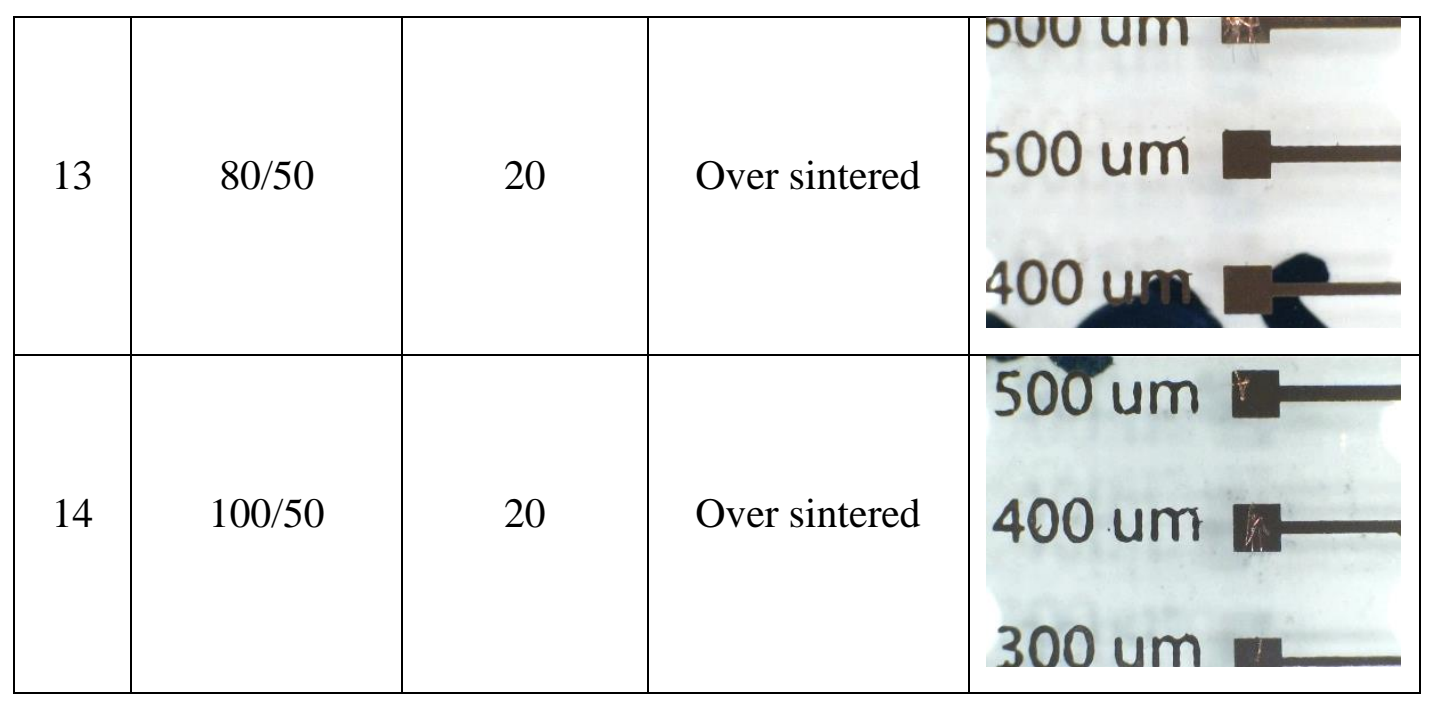

Figure 5.2 shows the SEM images of the sintered samples with NIR (at 50\% for 40 seconds), which introduced no significant change of the printed copper films. Overall, there is no noticeable change in the microstructure (Fig. 5.2 a, b and c) which explains the poor conductivity obtained with NIR sintering. For the $100 \mathrm{~N}$ ink, there is no sign of intra nano-particle sintering, but there is a clear fracture in the film, which would have a detrimental impact on cross film conductivity. The 50N50M and 20N80M inks also show similar microstructure with no evidence of the nano -particles sintering to the micro particles. The attempt to NIR sinter the films has therefore been unsuccessful as the exposure to the NIR sintering has negligible impact on the film microstructure. 
Chapter 5

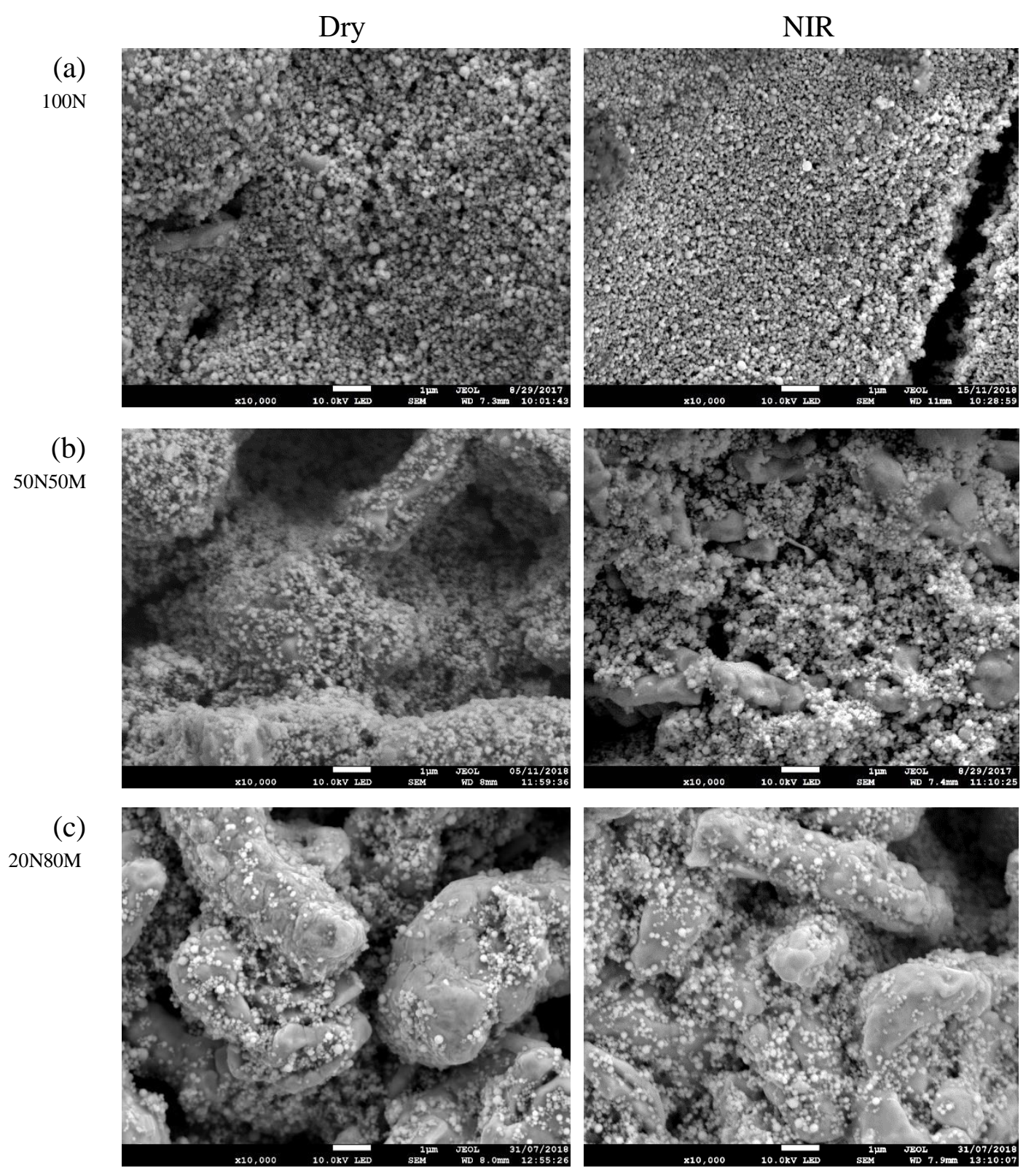

Figure 5. 2: SEM images of Dry only vs NIR sintering at $50 \%$ \& $40 \mathrm{~s}$. 


\subsection{Discussion}

The experimental study has been unable to identify a set of conditions where either laser or NIR sintering can be used to sinter any of the three materials rapidly. In some respects, this a failure of the hypothesis that it is possible to sinter nano-micro copper inks using alternative energy sources. This source would provide processes which sit between the ultra-rapid, but challenging to control, broad-spectrum white light sintering and the reliable but slow thermal sintering under controlled conditions.

In terms of laser sintering, there is evidence of success in the literature when sintering thin films of pure nano-copper materials on polymer substrates, but this has not been achieved in this instance with thick films and glass substrates. At the lower powers possible, the film is unaffected. Still, the laser has a detrimental impact on film integrity once the power is increased with the film exhibiting cracking, blistering and delamination. The limited number of samples did not allow the study to be expanded to other laser sources and timescales, but this should be examined further in order to develop the possibility of sintering via laser.

The large quantity of energy to which the film is exposed causes localised surface melting faster than the energy can be conducted into the film in order to sinter through the film. Increasing the timescales and lowering the power should allow the localised surface energy absorption to be reduced with greater conduction into the bulk of the film. These longer timescales would need to be balanced with the timescales of oxidation, but as most laser pulses are in the pico-second to milli-second range [167] then this will be minimised. The broad-spectrum white light sintering which occurred over 100s of milliseconds could produce some sintering with minimal oxidation.

The negative results for the near infra-red sintering show there is a lower energy density which is required to sinter the materials at the relevant timescales. The lack of changes in microstructure which were observed suggests that the constant lamp output energy density of $360 \mathrm{~kW} / \mathrm{m}^{2}$ in the infra-red region is insufficient to cause sintering. 


\section{5: Conclusion}

The study has shown that the laser system used is not conducive to sintering any of the thick film copper inks on the glass substrate. In most instances, a non-conductive film is produced, which exhibits severe damage when the power of the laser is increased. Further work is recommended on using lower intensity, longer pulse width laser systems.

This NIR technique was found ineffective to sinter the copper inks used in this study. The ideal NIR exposure conditions are linked to the actual power, the engineering power and film thickness, and this imposes a critical interaction between the processing and the materials techniques as the copper inks are highly responsive to any of the sintering parameters. 


\section{Chapter 6: Blended conductive materials}

\subsection{Introduction}

This chapter will present the work on mixed ink of copper nano-micro particles and sliver nano-particles with low sintering temperatures. There were two primary drivers in this work, one practical and one conceptually interesting. The practical reason was that with the demise of Intrinsiq materials ltd, further supplies of inks were not possible, so the volume of the stock was limited. The silver nano-particle inks were supplied by AGFA, the ORGACON nano-Silver inks feature very high conductivity with a low deposition and support high-resolution patterning.

The concept behind mixed inks was that since inks containing the lowest nano-particle ink $(20 \mathrm{~N} 80 \mathrm{M})$ seemed to less sensitive to the sintering environment, it was postulated that silver nano-particles could be used to fuse the micro copper particles together and form the conductive path through the copper/silver mixture. With sliver dominating the nano-particles sizes $30-80 \mathrm{~nm}$, their insensitivity to silver would allow a more stable matrix to be used. Literature has also highlighted work on mixing copper and silver where the surface copper atoms serve as reducing agents for the silver ions, in a simple term creating a silver shell on the copper core [168].

\section{2: Optimization of mixed inks}

The 20N80M copper ink exhibited the least oxidation under $\mathrm{H}_{2} / \mathrm{N}_{2}$ atmosphere sintering, Chapter 4 . In addition, the good conductivity this material was significantly stable and showed no drift in resistance after four weeks of sintering, as it contained the lower ratio nano to micro copper particles compared to the other materials in this study this was chosen as the base copper ink

The sintering time and temperature are to set within the operational window of 20N80M copper ink and silver nano-ink. The mixing was carried out manually with ratios of silver- copper ink. Figure 6.1, Table 6.1.

Table 6. 1 Mixed ink ratio

\begin{tabular}{|c|c|c|c|c|}
\hline$\%(\mathrm{~g})$ & $\mathrm{Ag}(\mathrm{g})$ & $\mathrm{Cu}(\mathrm{g})$ & $\begin{array}{c}\text { Total } \\
\mathrm{W}\end{array}$ & $\% \mathrm{Ag}-\mathrm{Cu}$ \\
\hline $25 \mathrm{Ag} 75 \mathrm{Cu}$ & 7.59 & 22.71 & 30.30 & 25 \\
\hline $54 \mathrm{Ag} 46 \mathrm{Cu}$ & 20.37 & 17.24 & 37.61 & 54 \\
\hline $75 \mathrm{Ag} 25 \mathrm{Cu}$ & 30.17 & 9.85 & 40.02 & 75 \\
\hline
\end{tabular}


$25 \mathrm{Ag} 75 \mathrm{Cu}$

$\mathrm{wt} \%$

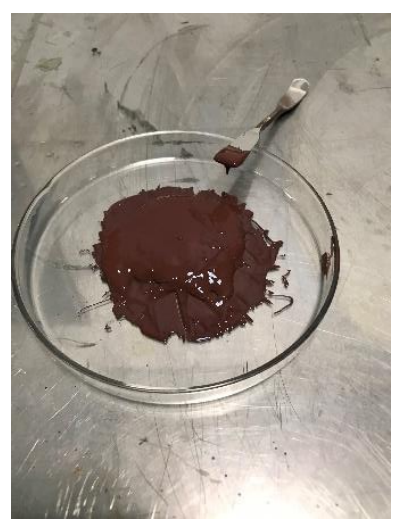

$54 \mathrm{Ag} 46 \mathrm{Cu}$

$\mathrm{wt} \%$

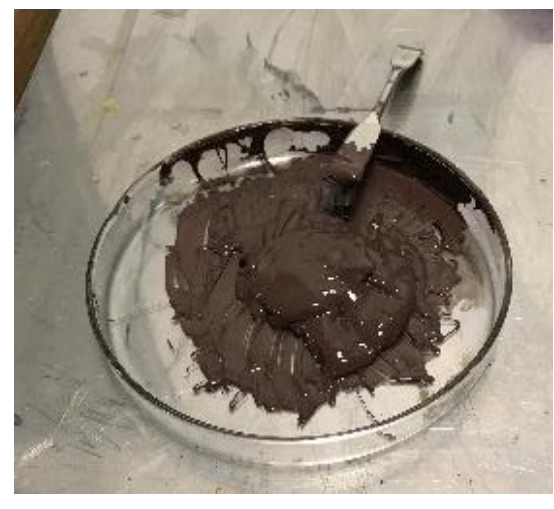

$75 \mathrm{Ag} 25 \mathrm{Cu}$

$\mathrm{wt} \%$

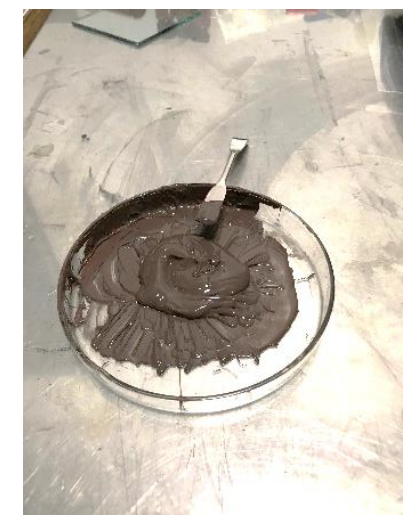

Figure 6. 1: Mixed ink (silver-copper)

\section{3: Resistance measurement (mixed film conductivity)}

The line resistance was measured after sintering at each temperature under the $\mathrm{H}_{2} / \mathrm{N}_{2}$ atmosphere. Figure 6.2 shows the line resistance performance of each blended ink and the silver and copper $(20 \mathrm{~N} 80 \mathrm{M})$ individually. The printed were initially processed at the optimum which had been found for copper material, namely 30 minutes at $250{ }^{\circ} \mathrm{C}$. The samples were also processed at the silver ink supplier recommended a sintering temperature of $275^{\circ} \mathrm{C}$ for 10 minutes.

In all instances, increasing silver content results in a lower line resistance, Figure 6.2. The copper 600 -micron line resistance is $4.21 \Omega$ while the silver is $0.16 \Omega$, and there is a linear reduction in the resistance as the silver content increases. Part of this may be related to the intrinsically lower resistivity of the silver compared to the copper $\left(\rho_{\mathrm{Ag}}=\right.$ $\left.1.59 \times 10^{-8} \Omega \mathrm{m}, \rho_{\mathrm{cu}}=1.68 \times 10^{-8} \Omega \mathrm{m}\right)$, but this represents only a $5 \%$ increase in resistance. The inference which can be drawn from this is that the sintering of the copper provides a less conductive feature than which is produced from the silver ink and this is likely to be a result of the oxidation of the copper material in the wet, during the drying or during the sintering process. This is independent of the printed line structure size, Figure 6.3 


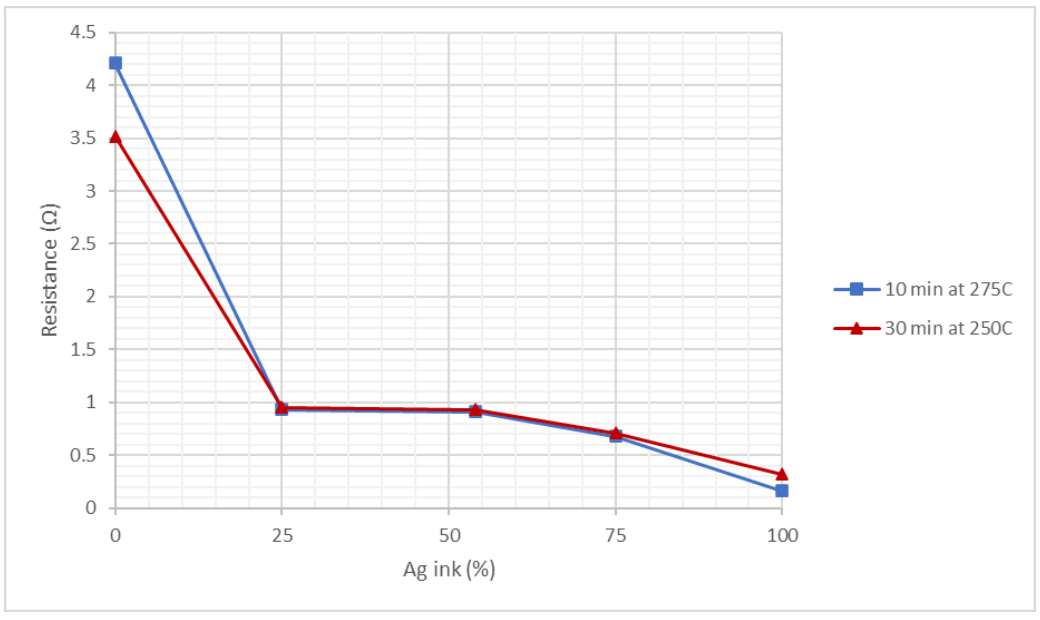

Figure 6. 2: The impact of silver content on the resistance of the 600-micron line

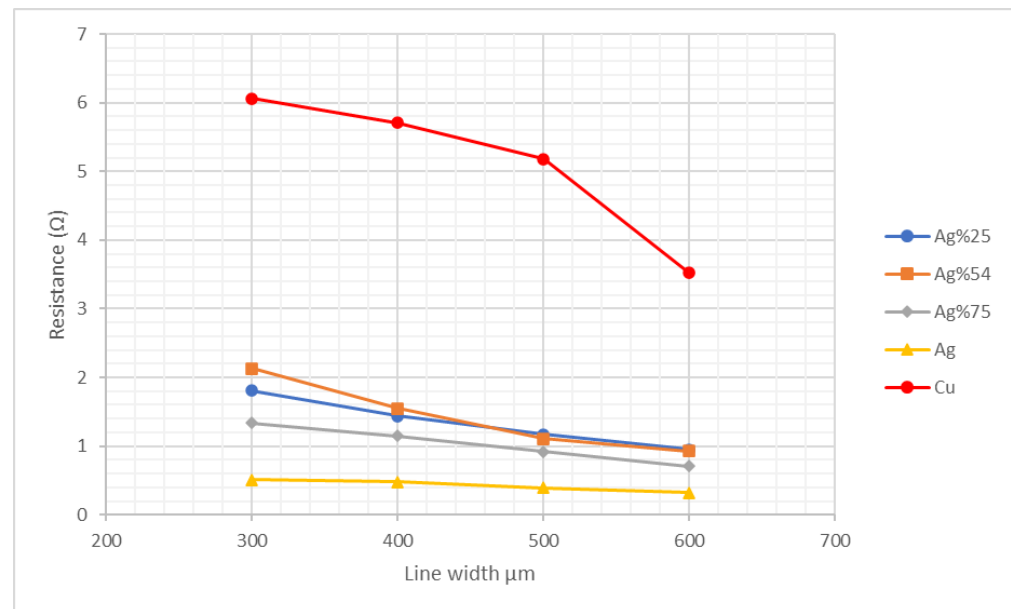

(a) $250^{\circ} \mathrm{C}$ for 30 mins

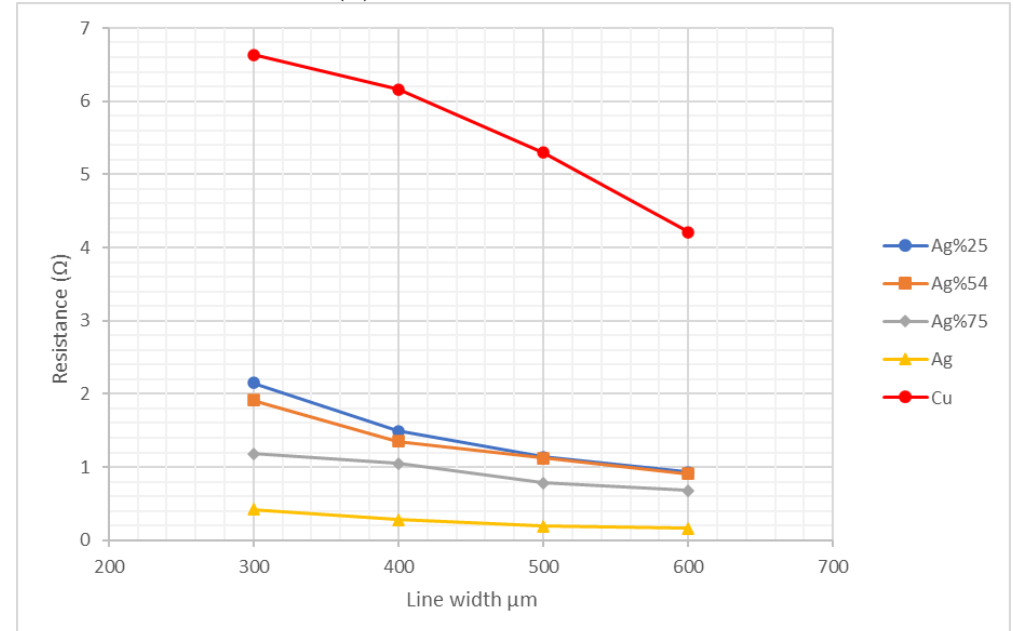

(b) $275^{\circ} \mathrm{C}$ for $10 \mathrm{mins}$

Figure 6. 3: Mixed ink line resistance $\mathrm{H}_{2} / \mathrm{N}_{2}$ condition compare to $\mathrm{Ag}$ and $\mathrm{Cu}$. 
The absolute line resistivity was also calculated after measuring the film thickness by employing Taylor Hobson S-28 profilometer. The absolute resistivity obtained with the mixed ink for the $600-300 \mu \mathrm{m}$ line width is summarized in Figure 6.4.

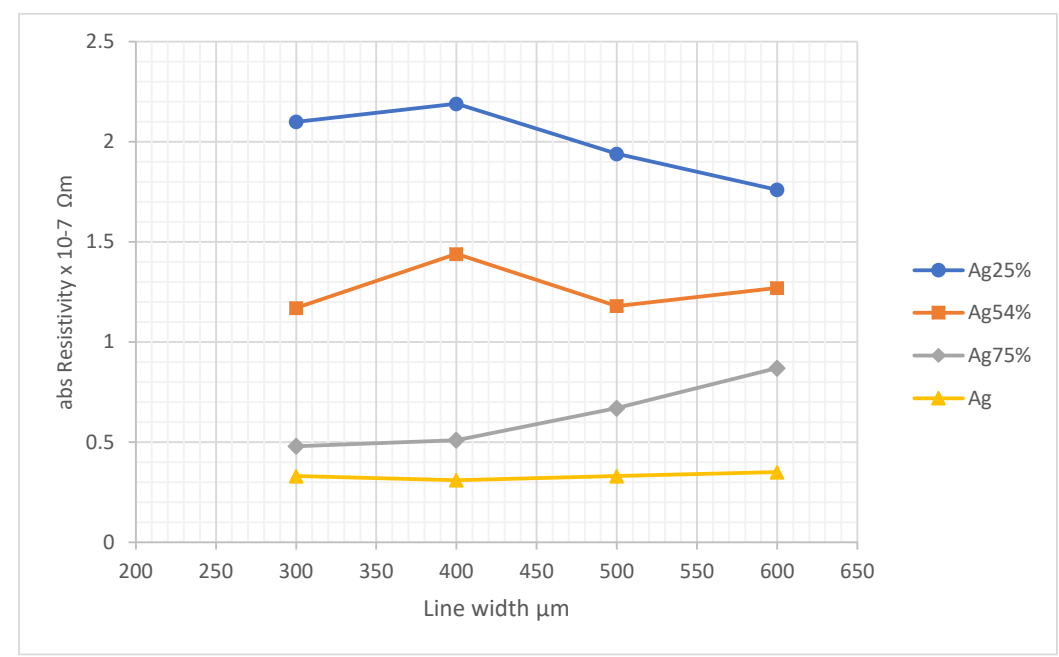

Figure 6. 4: Mixed ink absolute resistivity sintered under $\mathrm{H}_{2} / \mathrm{N}_{2}$ condition compare to silver nano-particle resistivity

The absolute resistivity obtained with blended ink compared to the bulk silver resistivity and also compared to the best resistivity obtained with the thermal condition in Table 6.2. The measured absolute resistivity is between 10-12 times higher for the blended ink with \%25 of silver, than the bulk silver and around 2-3 times higher with $\% 75$ silver. The inclusion of the copper, therefore, tends to have a detrimental impact on the conductivity of the sintered film.

Table 6. 2: Absolute resistivity relationship for each material for the $500 \mu \mathrm{m}$ line (Mixed ink related to bulk Silver and best obtained with thermal)

\begin{tabular}{|l|c|c|c|}
\hline \multicolumn{1}{|c|}{$\begin{array}{c}\text { Ink } \\
\text { wt\% }\end{array}$} & $\begin{array}{c}\rho \text { (abs Resistivity) } \\
* 10^{-7} \Omega \mathrm{m}\end{array}$ & $\begin{array}{c}\text { Related to Bulk Silver } \rho \\
1.59 * 10^{-8} \Omega \mathrm{m}\end{array}$ & Related to the best thermal $\rho$ \\
\hline $\mathrm{Ag} 25$ & 1.94 & $10-12$ & $0.5-1$ \\
\hline $\mathrm{Ag} \mathrm{54}$ & 1.18 & 8 & $0.4-1$ \\
\hline $\mathrm{Ag} 75$ & 0.671 & $2-3$ & $0.1-1$ \\
\hline
\end{tabular}




\section{4: Contact resistance of the sintered samples}

All samples remained intact during the sintering process, and therefore the contact resistance was measured using the TLM procedure for mixed ink samples sintered under $\mathrm{H}_{2} / \mathrm{N}_{2}$. The trends observed in sheet and contact resistance in the thermal sintering section are generally repeated with mixed ink samples, Figure 6.4. The similarly in contact resistance $\mathrm{Ag} \% 54$ and $\mathrm{Ag} \% 75$ ink resulted according to the high percentage of silver - copper which generally provided the lowest contact resistance at the sintering temperature of $250^{\circ} \mathrm{C}$ for 30 minutes and higher contact resistance with the higher percentage of copper-silver (Ag \%25).

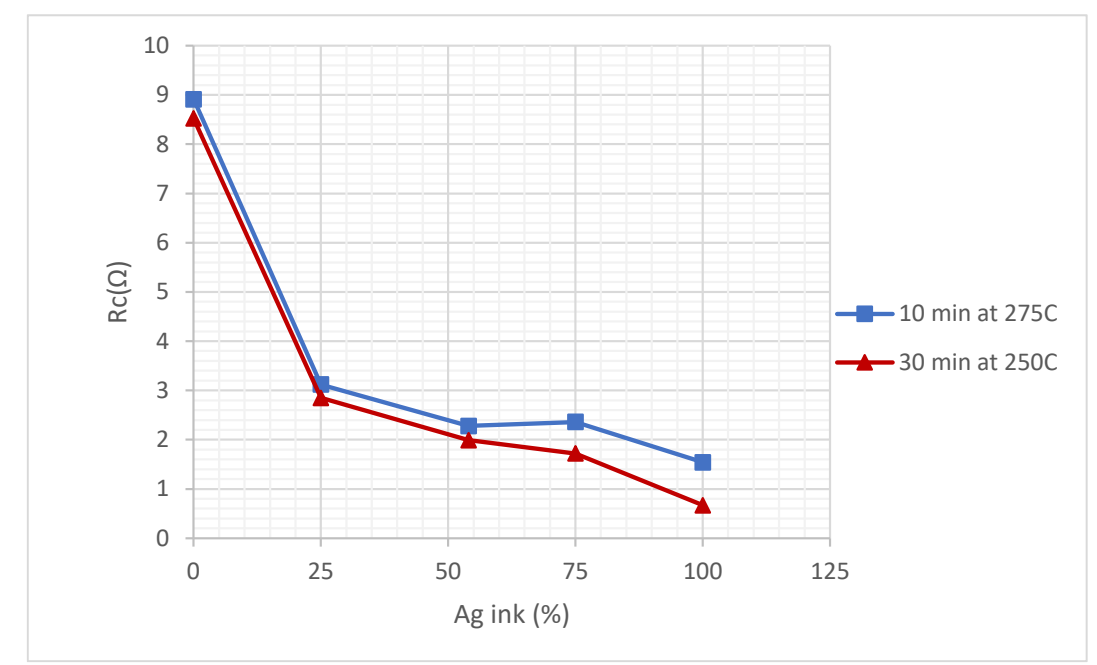

Figure 6. 5: Mixed ink contact resistance $\mathrm{H}_{2} / \mathrm{N}_{2}$ sintered related to pure silver and 20N80M copper.

Contact resistances for the micro/nano-particle copper inks are around six times higher than observed with the nano-silver, Figure 6.5, with lower silver content leads to higher contact resistance. Even the lowest silver content $(\mathrm{Ag} \% 25)$ lowers the contact resistance appreciably with further smaller reductions as the silver content is increased ( $\mathrm{Ag} \% 54$ and $\mathrm{Ag} \% 75)$. It is postulated that the lower contact resistance achieved with increasing silver content is attributed to the particle size $(30-50 \mathrm{~nm})$ and this is likely to be due to the reduced contact area at the ink/substrate interface [21]. 


\section{5: Sheet resistance by 4-point probe $\mathrm{H}_{2} / \mathrm{N}_{2}$ sintered samples}

The sheet resistance obtained from the mixed ink process is displayed in Figure 6.6. These results show similar trends to those observed with the contact resistance and line resistance. The inclusion of the smallest percentage of silver results in a significant improvement in the conductive performance of the material, while higher percentages tend towards the conductive properties of the pure silver ink.

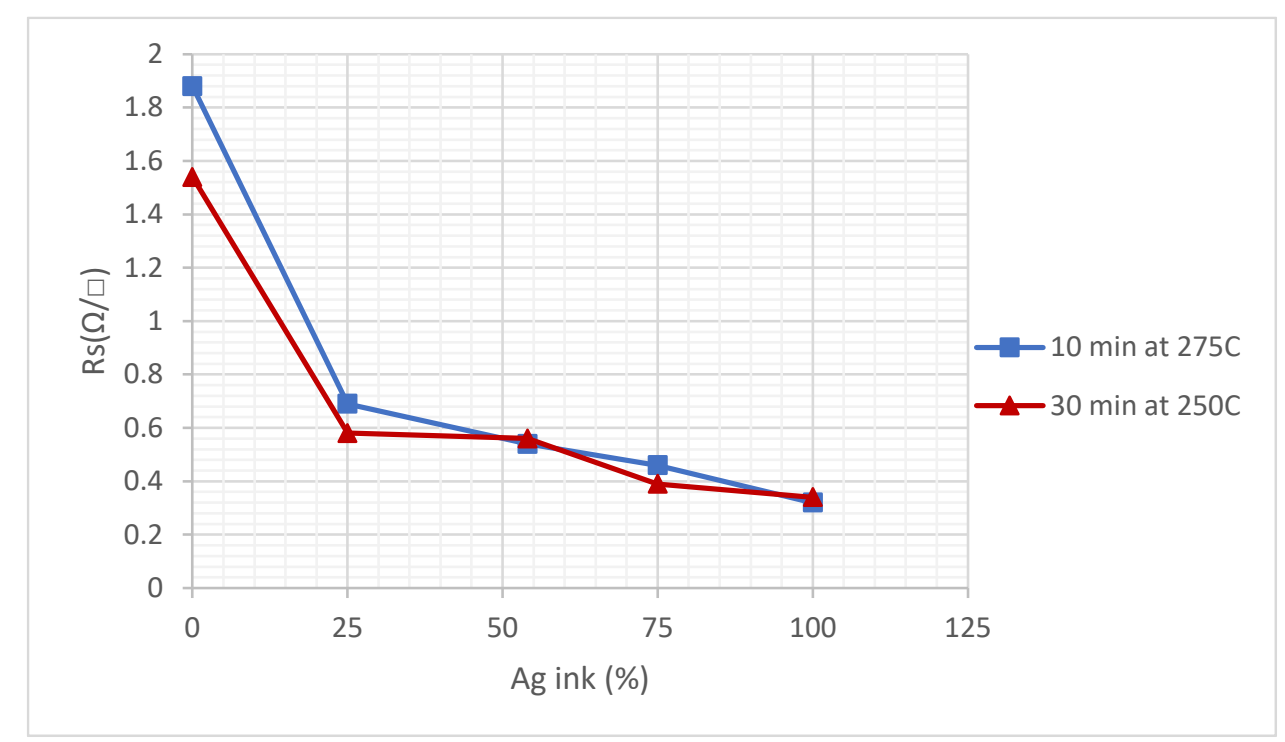

Figure 6. 6: Mixed ink, sheet resistance $\mathrm{H}_{2} / \mathrm{N}_{2}$ sintered related to pure silver and 20N80M copper

The lowest sheet resistance achieved with the blended inks was $(0.39 \Omega / \square)$ at $250^{\circ} \mathrm{C}$ for 30 minutes with ink contains $\mathrm{Ag} \% 75$, which is only slightly higher than that of the pure silver ink.

\section{6: Adhesion test}

The introduction of the silver particles into the ink have clearly shown an improvement in the conductive performance of the sintered film. This improvement in electrical performance, however, comes at the expense of the physical adhesion characteristics of the film, Table 6.3. 
Table 6. 3: Adhesion tape test results for each mixed metal ink sintered at $250^{\circ} \mathrm{C}$ for 30 minutes

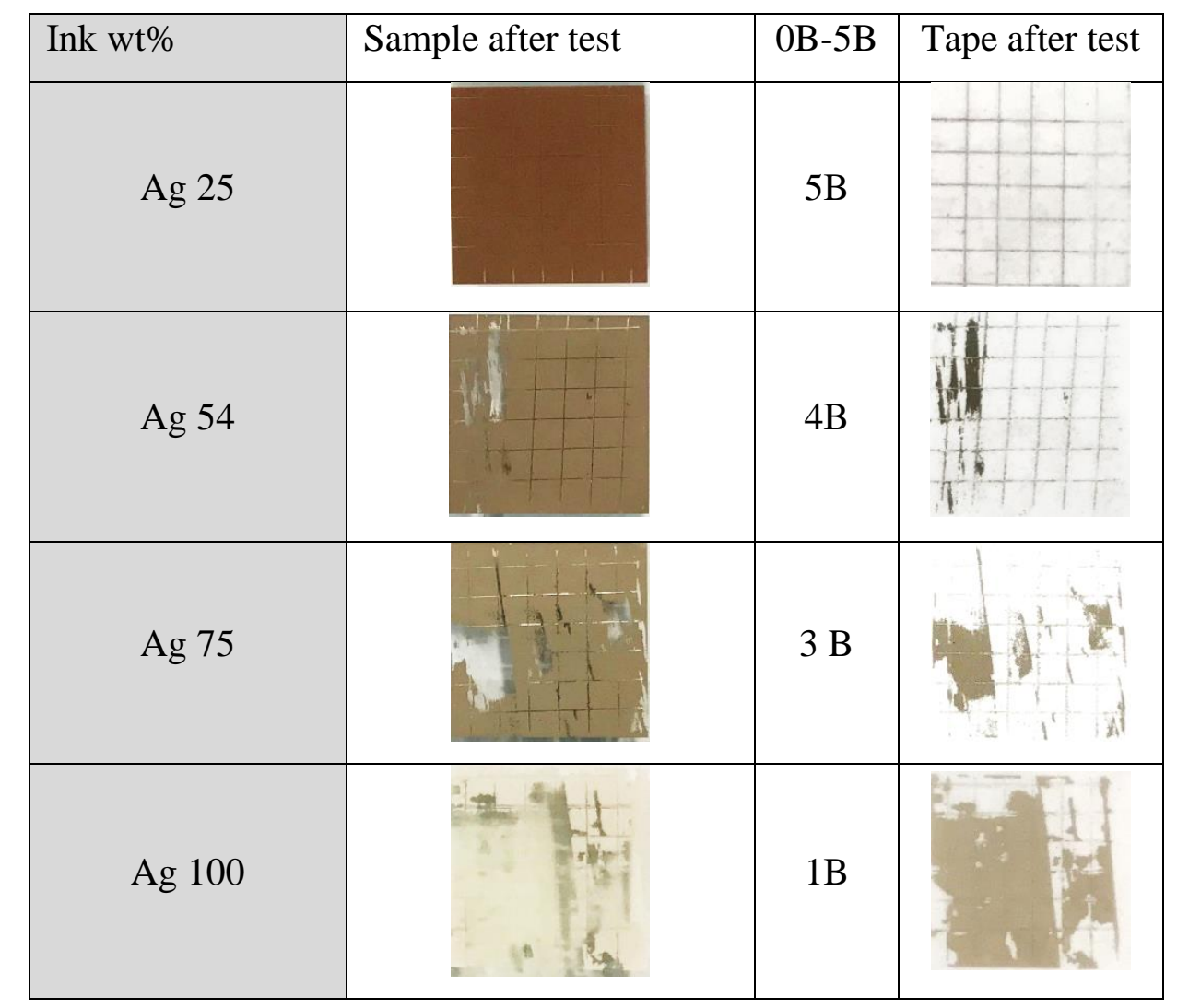

At $25 \%$ silver content, the adhesive performance of the ink is mostly unaffected with a small increase in the area removed as the silver content is increased to $54 \%$ silver. As the silver content is further increased to $75 \%$, more of the film is removed by the tape until the pure silver film exhibits more or less complete delamination under testing. Where delamination occurs, the sintered film is removed, ultimately indicating failure at the film / FTO interface and not within the film. This behaviour is similar to that observed with the nano copper ink where the sintering of the copper nano-particles results in a reduction in adhesion, Table 3.2. It was therefore postulated that the sintering of the nano-particles and the formation of a denser conductive film has a detrimental impact on the ink substrate interface. In order to further examine this, the film microstructure was investigated. 


\section{7: Mixed ink material microstructure}

Selected samples were examined using SEM to examine the surface morphologies. The sintered silver provides a uniform distribution of nano-particles across the surface of the film, and the interparticle melding is clearly observed at zooms into the nanoscale, Figure 6.7 (a). As previously seen, the sintered film produced 20N80M copper ink is characterized by large micro particles to which the nano-particles are attached then there exist voids in the film due to the lower packing density of the micro particles, Figure 6.7 (b). The introduction of $25 \% \mathrm{Ag}$ into the ink results in a denser film with fewer large voids in the film as the nano silver fills the inter-micro particle gaps. This decrease in porosity results in the increase in conductivity observed. As with the nano copper, closer inspection shows that the nano-particles do not appear melted into the micro particles but remain as discrete nano-particles on the surface of the micro particles, Figure 6.7 (c).

$\mathrm{Ag}$

(a)

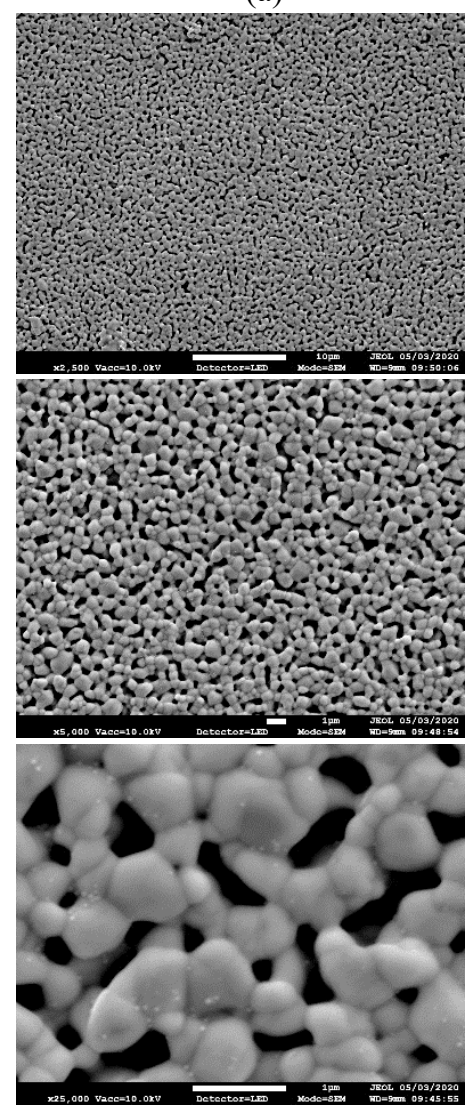

$\mathrm{Cu}(20 \mathrm{~N} 80 \mathrm{M})$

(b)

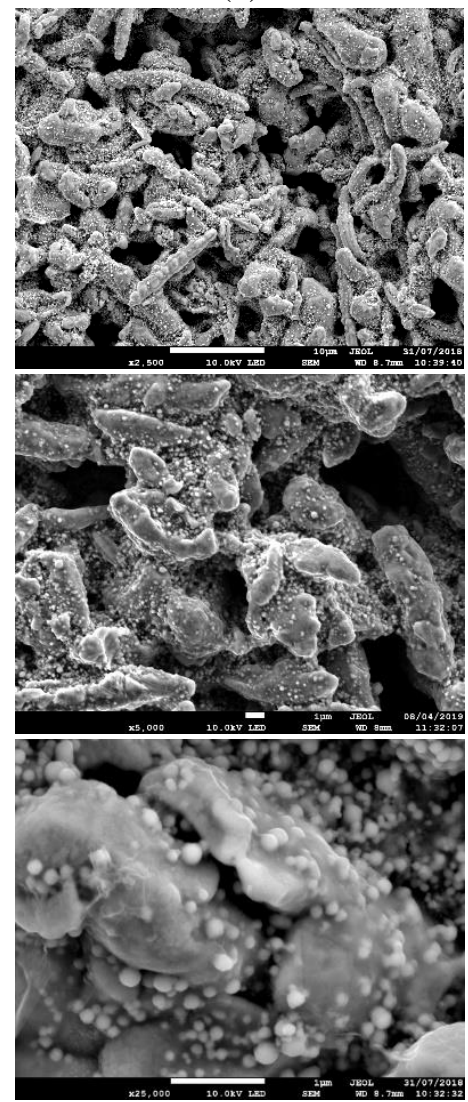

$\operatorname{Ag} 25 \%$

(c)

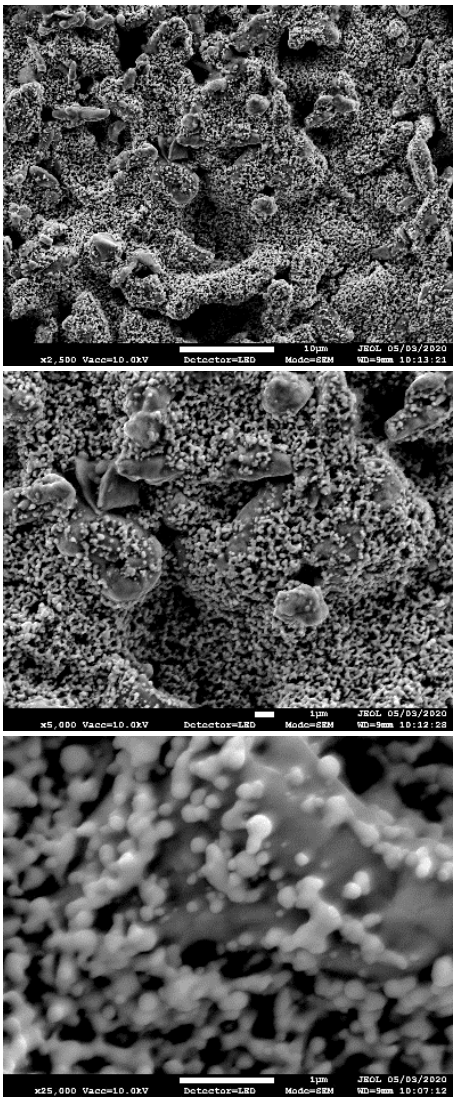

Figure 6. 7 .SEM Nano-silver, 20N80M copper with the mixed ink of Ag 25\% 
Increasing the proportion of nano-silver in the ink results in fewer voids in the sintered film with the surface of the copper micro particles, being further covered by the silver nano-particles, Figure 6.8. With the increase in the number of nano-silver particles, there is evidence of interparticle necking which would readily allow for particleparticle conduction. The reduction in voids and the silver nano-particle sintering would both provide a positive impact on the conductivity. Even at the highest nanosilver proportion, a continuous film is not present, and thus it can be concluded that charge transfer occurs through both the silver and copper

(a) $25 \% \mathrm{Ag}$
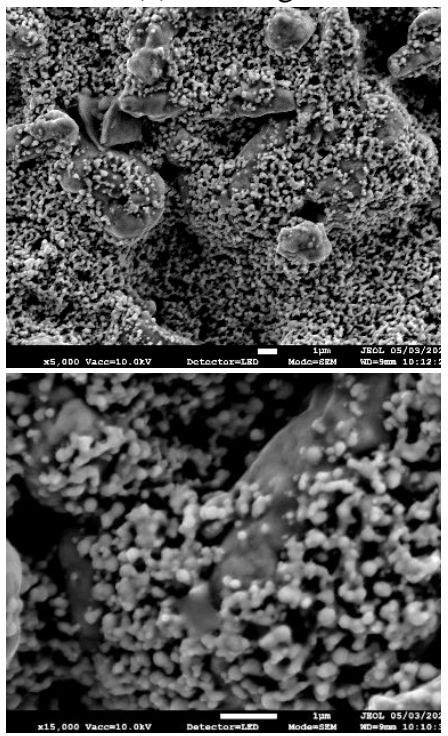

Figure 6. 8: SEM Nano-silver, 20N80M copper ink blended with (a) 25\%, (b) 54\% (b) $54 \% \mathrm{Ag}$

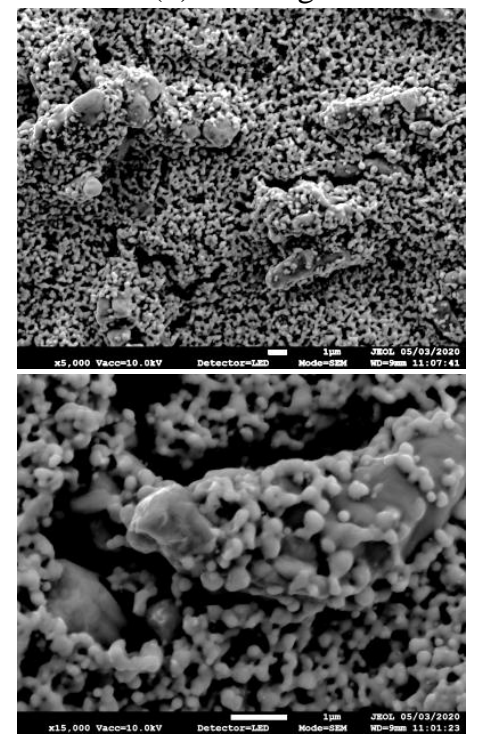

(c) $75 \% \mathrm{Ag}$

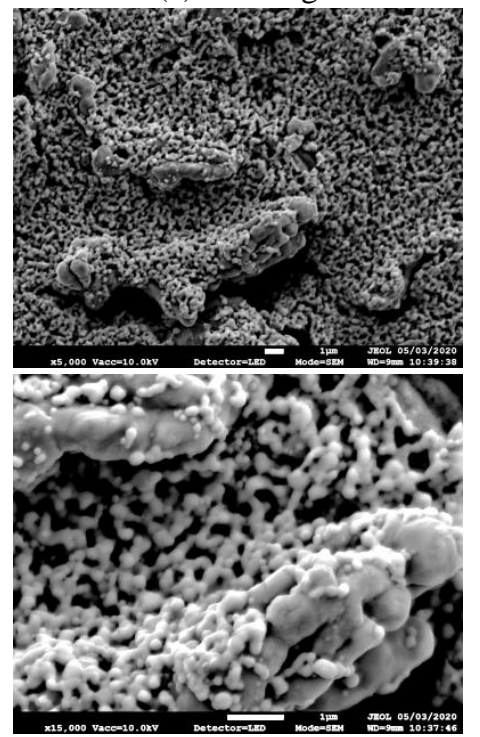

and (c) $75 \% \mathrm{Ag}$

\subsection{Mixed ink Energy-dispersive X-ray spectroscopy (EDS) examination}

An EDS of the sintered films was used as a means of investigating the atomic composition of the films. When the silver nano-particles are sintered under ambient air conditions, there is little oxidation observed on the film with the spectra being dominated by the presence of silver as expected, Figure 6.9. There is a small quantity of carbon which is associated with contamination and any residual binder which may still be present post the sintering process. 


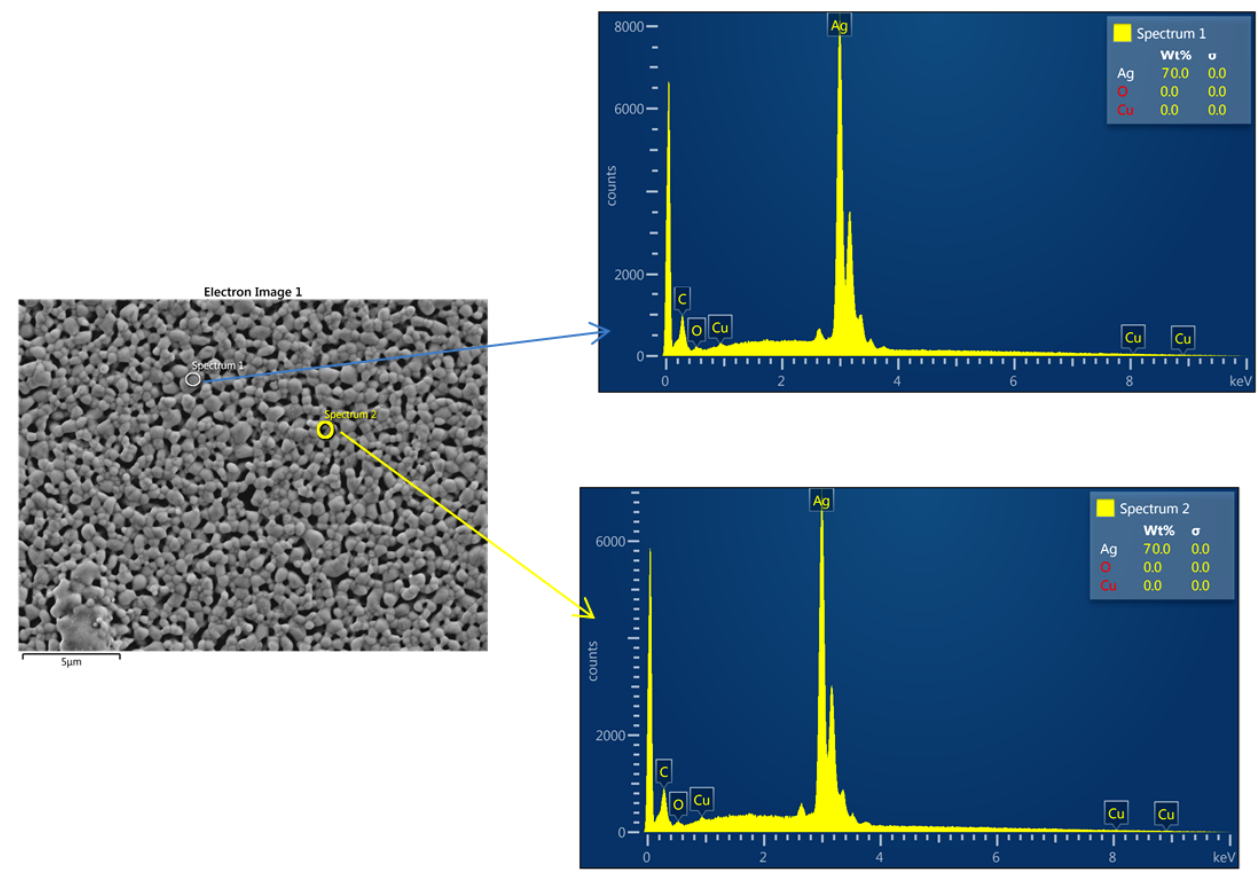

Figure 6. 9 EDS: Ag ink sintered under ambient condition

With the $25 \% \mathrm{Ag}$ film, both copper and silver are present, although the relative quantity of each varies considerably due to the distribution for the nano silver on the micro copper particles. Figure 6.10.

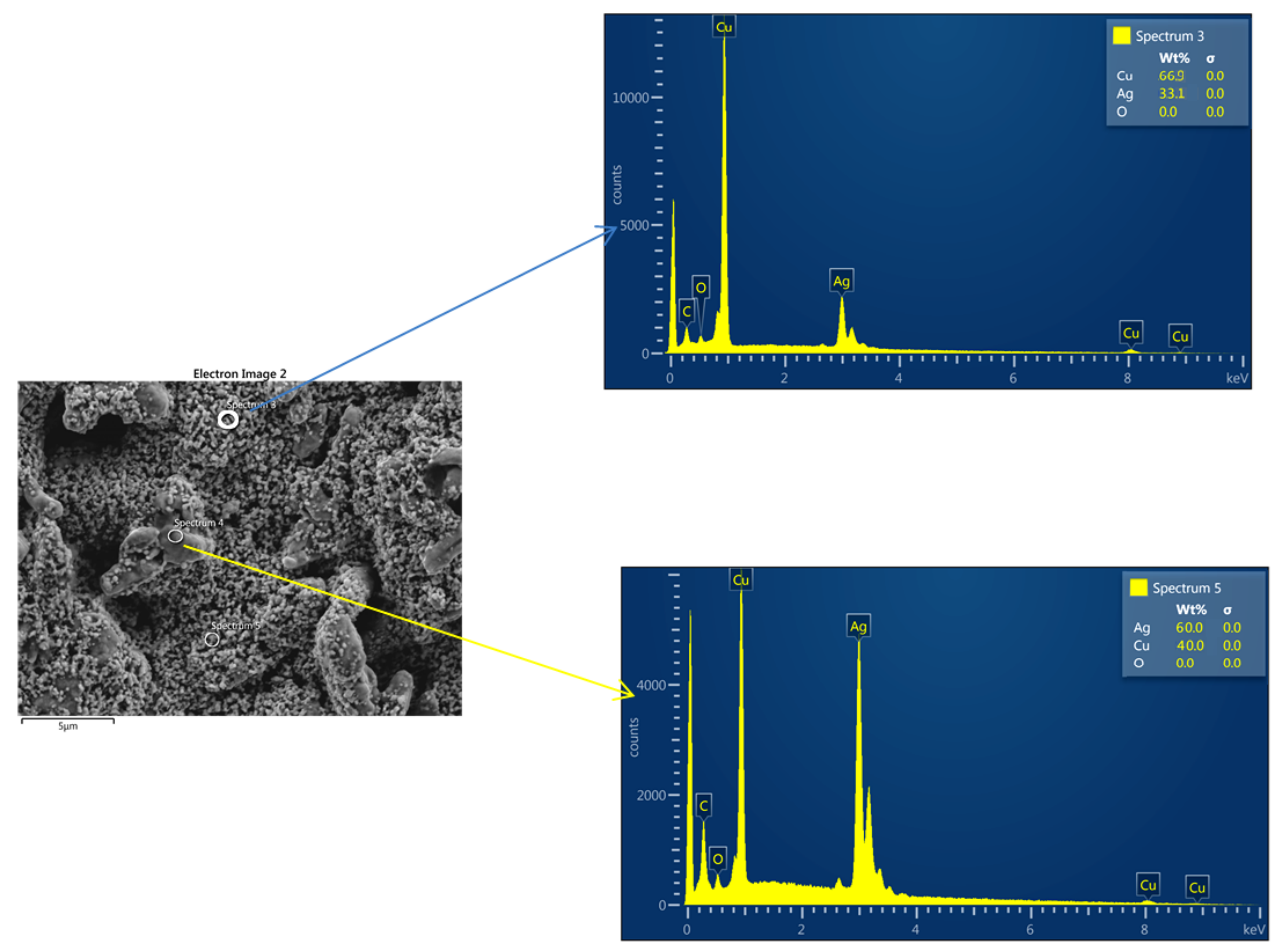

Figure 6. 10: EDS of $\mathrm{Ag} \% 25$ ink sintered under $\mathrm{H}_{2} / \mathrm{N}_{2}$ condition 
Increasing the silver content to $54 \%$ results in similar distributions of silver and copper with the discrete particulate nature of the metals, creating localised differences in relative quantity, Figure 6.11. Again, the oxygen levels are negligible which suggests that the copper has not been oxidised with a subsequent adverse effect on the oxygen

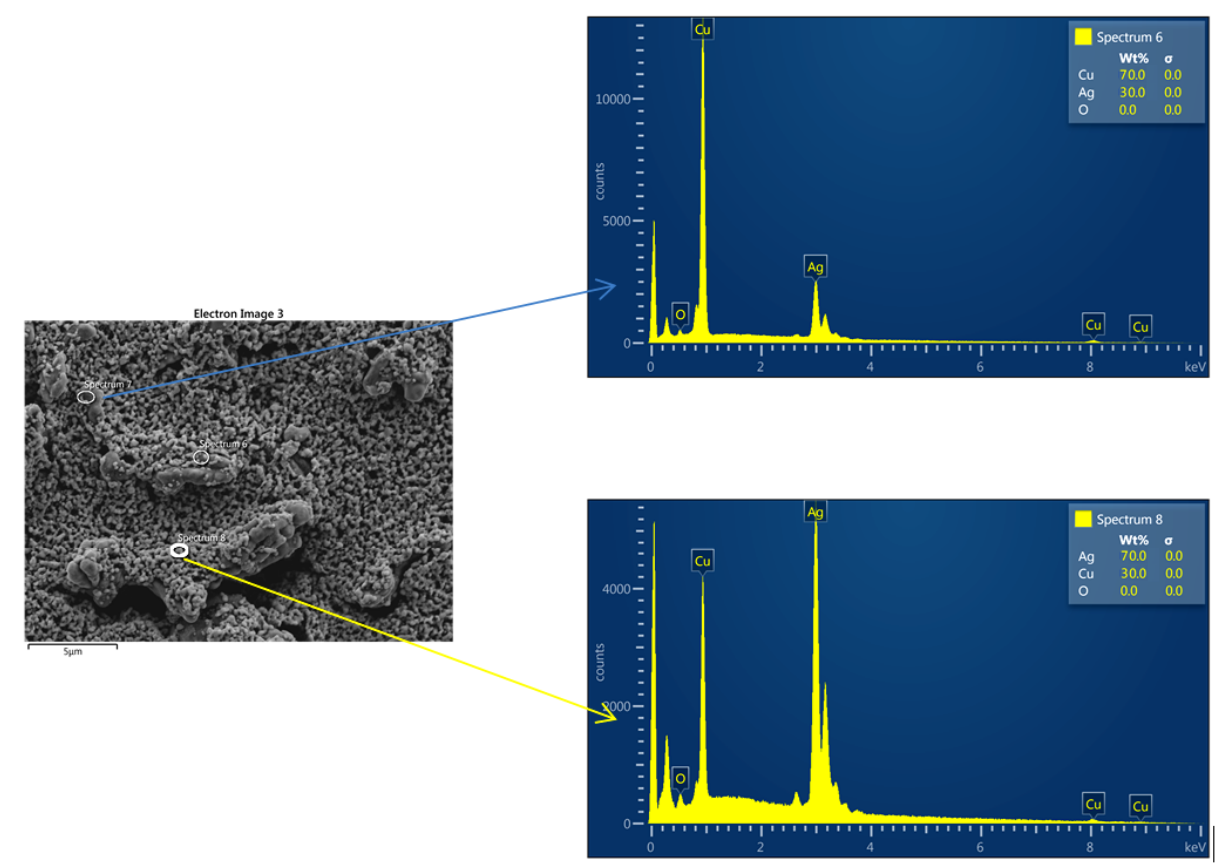

Figure 6. 11: EDS of $\mathrm{Ag} \% 54$ ink sintered under $\mathrm{H}_{2} / \mathrm{N}_{2}$ condition

As the silver content increases to $75 \%$, the distribution mix of the silver nano-particles and micro copper becomes more even and there remains little observed surface oxygen, Figure 6.12

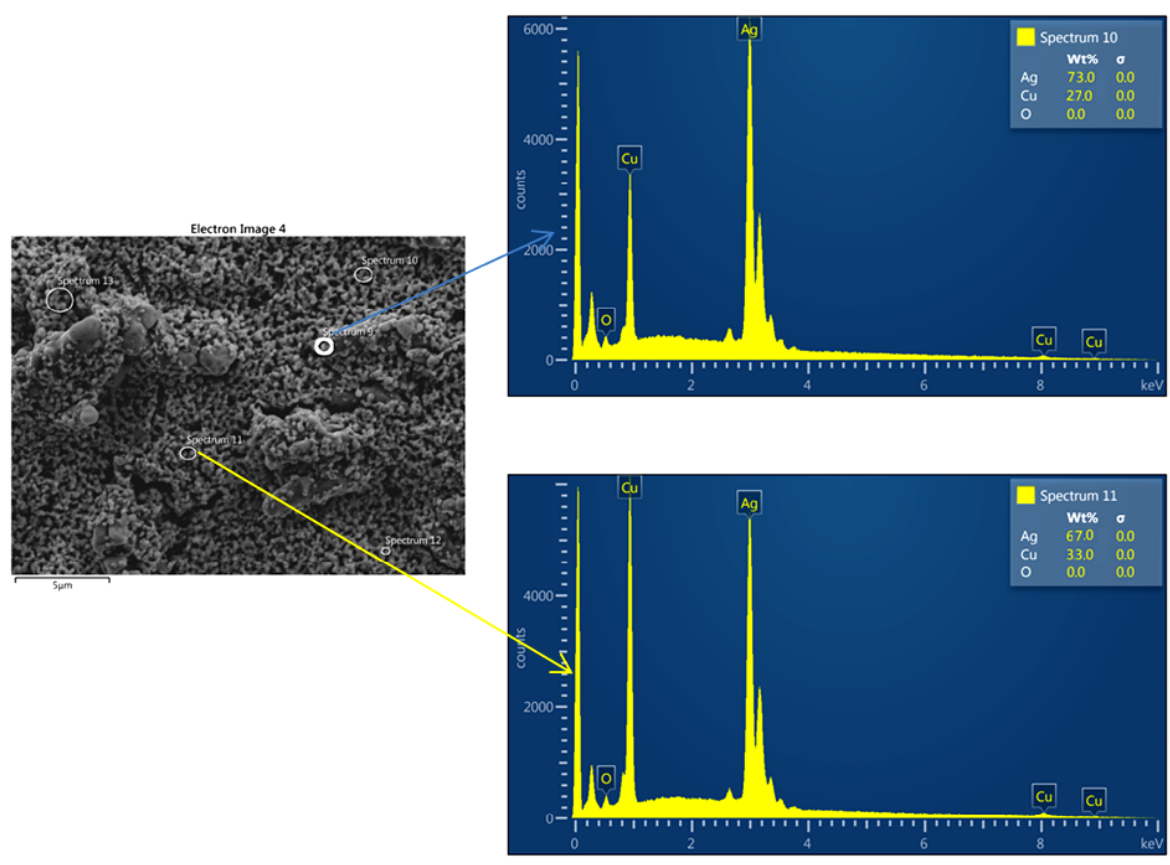

Figure 6. 12: EDS of $\mathrm{Ag} \% 75$ ink sintered under $\mathrm{H}_{2} / \mathrm{N}_{2}$ condition 


\section{9 (XRD) examination of mixed ink}

$\mathrm{X}$-ray diffraction was used to identify changes in the structure and possible oxidation due to the sintering process. Figure 6.13 shows the diffraction peaks appearing at angles corresponding $2 \Theta 111,200$ and 220 for all mixed ink, highlighting the presence of copper and silver. The relative intensities of the peaks are in line with the ratio of the silver and copper components. No additional peaks are visible due to the sintering process, indicating little chemical change during the sintering process.

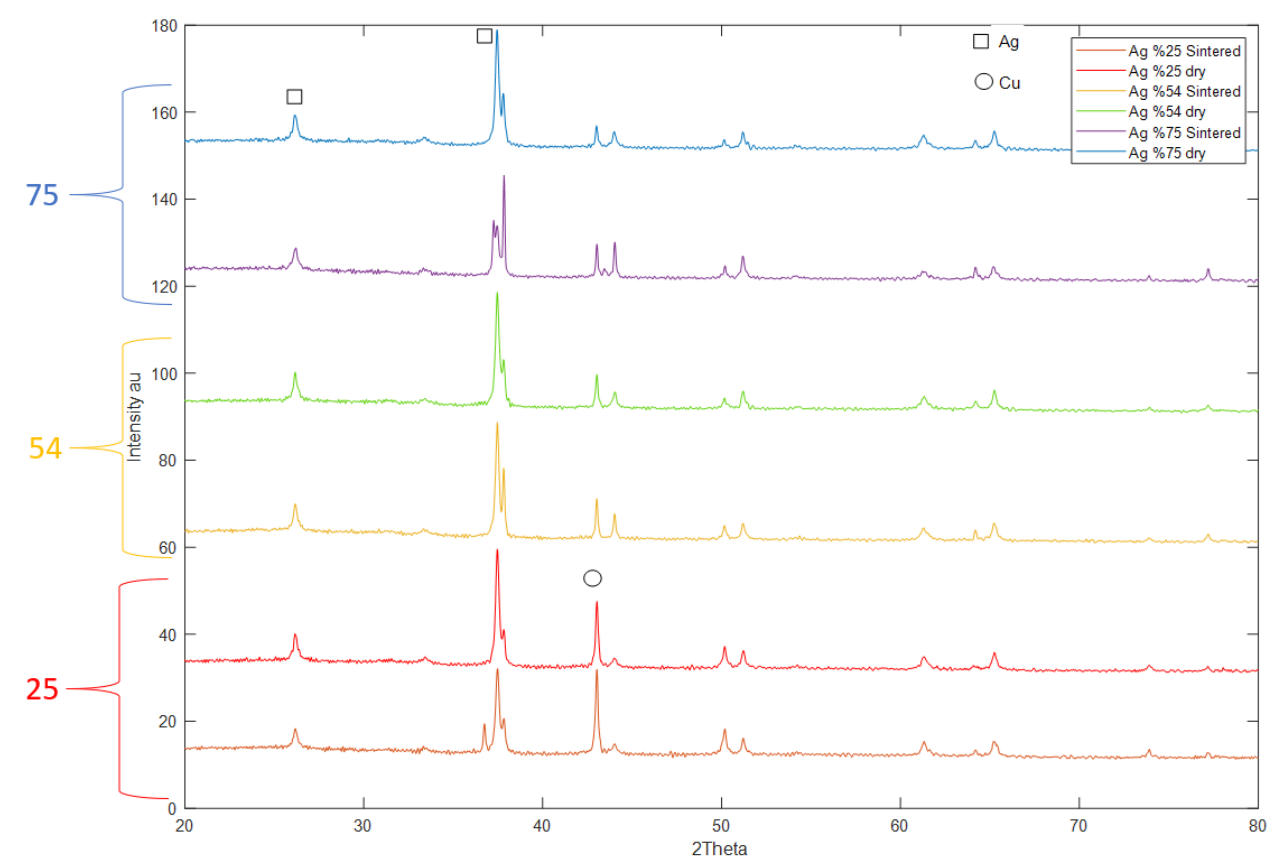

Figure 6. 13: XRD response of each mixed metal ink before (dry) and after the sintering process for 30 mins in a $\mathrm{H}_{2} / \mathrm{N}_{2}$ environment for $250{ }^{\circ} \mathrm{C}$.

Examining the XRD of mixed copper-silver sintered films, highlights detailed film characteristics, Figure 6.14. Clearly present are the three characteristic copper peaks at $2 \theta$ values of $43.27^{\circ}, 53.42$ and 74.12 corresponding to (111), (200) and (220), respectively. These characteristic peaks confirm that a face-centered cubic (FCC) copper phase is present. There is also no evidence of the $\mathrm{Cu}_{2} \mathrm{O}$ phase at $36.6^{\circ}$, which indicates that minimal oxidation has occurred. Also, shown peaks at $2 \theta$ values of $26.2^{\circ}, 38.19^{\circ}$ and $44.22^{\circ}$, which indicative of silver, the layer of silver should provide extra protection to the copper against oxidation [168]. 


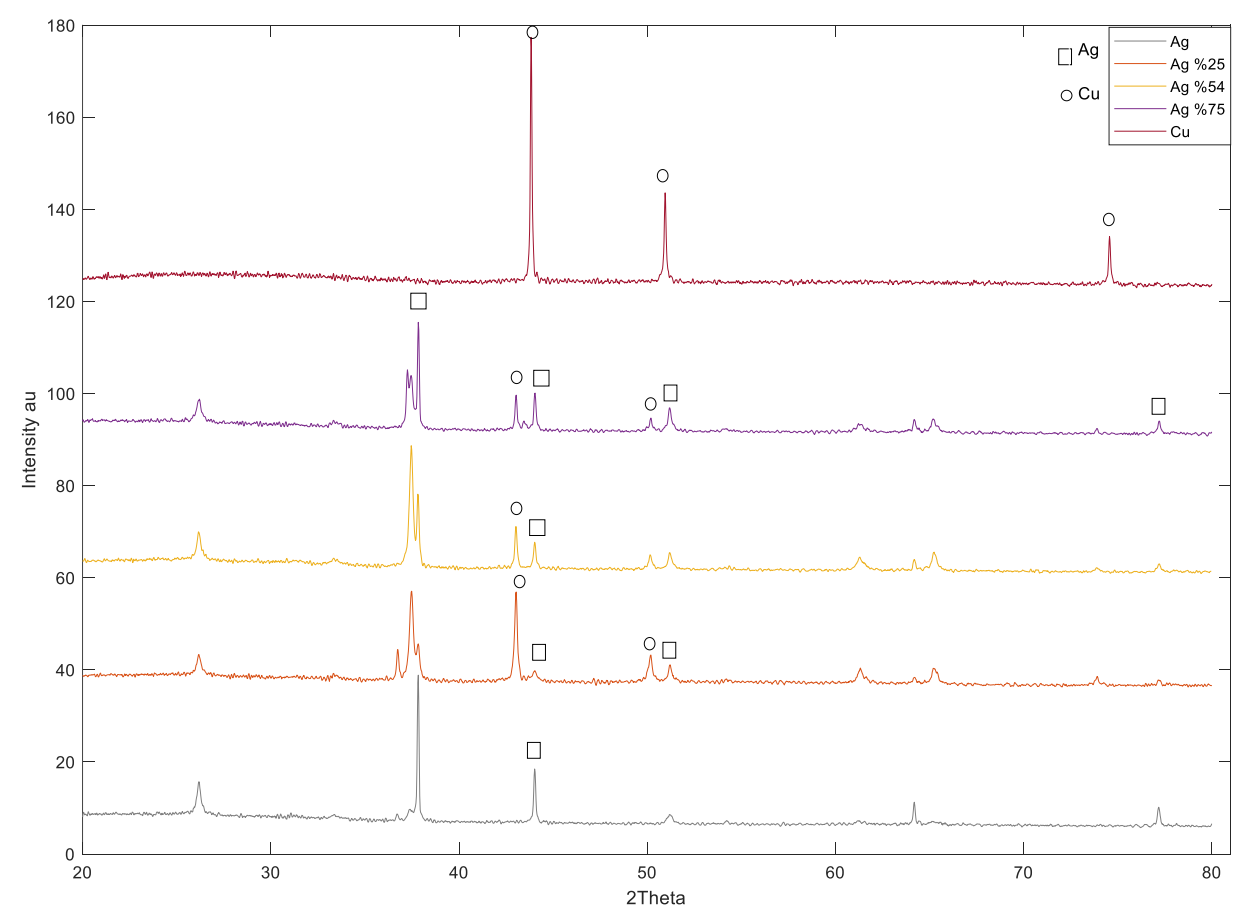

Figure 6. 14: XRD comparison of each film for each film sintered at $5 \% \mathrm{H}_{2} / \mathrm{N}_{2}$ for 30 min@250 ${ }^{\circ} \mathrm{C}$ in comparison to the unmixed material

\subsection{Discussion}

This chapter's study raises a number of discussion points which have scientific and application impact. Firstly, it has been demonstrated that it is possible to achieve a reduction in resistance, an improvement in the conductive performance, of a film by blending a relatively small quantity of nano silver ink into the micro/nano copper ink. The inclusion of $25 \%$ nano silver reduces the sheet and line resistance up to three-fold compared to the original micro/nano copper (Figure 6.2 to 6.5) without any apparent loss in adhesion. Thus, adding silver nano-particles provides an enhancement in conductivity without a penalty of loss of adhesion on FTO coated glass substrate. With the potential raw material cost reduction, then this provides a significant outcome of the study. There may be potential to further optimize the trade-off between conductivity and adhesion through further refinement, of the relative proportions, but this initial finding represents a successful outcome. 
In the copper micro/nano blends the presence of micro particles prevented visible sintering of the nano-particles to the micro particles. This was also observed with the copper ink where the individual nano-particles remained as discrete spherical particles on the micro particles as a result of sintering. However, when the nano-particle density increased such that they touch the nano-particles then meld into each other, Figure 6.7. Thus, the presence of the micro particles does not impede the sintering of the silver particles as was apparent with the nano copper blend ink. It is

The lack of oxygen/copper oxide observed in the EDS and XRD respectively raises the question of whether the presence of the silver plays a role in helping to prevent oxidation of the copper during sintering. This occurs even at low silver contents, and therefore it is unlikely that it is providing a protective layer since the nano-particle silver film on the surface of the copper micro particles is non-continuous, Figure 6.7. Although copper lies above silver in the reactivity series and would, therefore, be more likely to oxidize the more likely hypothesis is that the inherent reactivity of the nanoparticles means that they may react preferentially with any oxygen inhibiting any copper oxide formation.

\subsection{Conclusion}

The work completed in this chapter has shown the potential of copper/silver mixtures as a means for providing high conductivity, physically robust conductive structures on FTO coated glass. The study has been successful in creating a blended ink where a $25 \%$ addition of nano silver reduces the conductivity significantly while maintaining the potentially lower cost of the essential conductive material copper 


\section{Chapter 7: Discussion}

This thesis has concentrated on studying copper inks materials and their interaction with the sintering process. The study has highlighted many scientific and practical interesting issues which arise from sintering of thick copper films to FTO coated glass. This chapter brings the individual chapter discussions together in order to gather the overall implications of the findings.

\subsection{Performance of nano vs micro particle inks}

There is a clear indication that the $100 \%$ pure nano-particle copper ink shows improved electrical performance compared to the inks with a blend of micro and nano particles. Such a trend can be explained by examining the micro structure of the materials.

While the $100 \mathrm{~N}$ ink showed extensive inter particle melding during sintering, the relatively poor performance of the 50:50 nano-particle/ micro-particle blend (highest sheet resistance, largest ageing effect) suggests that there is an optimum blending ratio of nano / micro particles which balances the performance benefit of each constituent. It is postulated, and backed by the SEMs of the 20N80M materials, that the presence of an excessive quantity of micro particles disrupts the natural sintering tendency of the nano-particles as they are rapidly bound to larger surface area micro particles, leaving them open to oxidation.

There are a number of possible explanations for the improved adhesion observed with the micro / nano-particle inks compared to the pure nano particle ink. It could be due to greater physical interlocking between the micro particles and the FTO crystals on the substrate surface. Some surface oxidation of the larger micro particles may be beneficial in terms of providing oxide - oxide bonding between the copper oxide and the FTO. It cannot be attributed to any organic binder being present as the TGA profile (Figure 3.1) clearly shows that the TGA shows no further change in mass beyond $225^{\circ} \mathrm{C}$ for the micro particle materials. 


\subsection{Thermal sintering conditions}

There is considerable interaction between the ideal thermal sintering conditions (time environment and temperature) and the nano - micro particle blend. Where the nano particles form a significant contributor to the continuous conductive (50N50M) pathways, this impairs charge transfer through the particles. At lower concentrations of nano particles, a conductive pathway is ensured through micro particle contact and thus the impact of any nano particle oxidation is minimized. Evidence for this hypothesis can be seen in the absolute conductivity and in the increase in resistance seen with ageing, suggesting that there are some nano particles which are not fully sintered which are subject to oxidation. While the short-term stability of the copper inks has been shown to be good when sintered under the correct conditions, this needs to be examined over a longer timescale.

In terms of the performance under $\mathrm{N}_{2}$ environment compared to that in a reducing environment, it is encouraging that sintering can occur in a purely inert atmosphere, albeit with a penalty in terms of conductive performance. In some instances, this performance penalty may be tolerable given the relative ease of installation and operation of inter / reducing atmospheres.

\subsection{Photonic sintering}

The better performance of the photonic sintering with the thinner film is likely related to two primary mechanisms the depth of energy penetration and the thermal mass [169]. With the thinner film, the initial transformation to a highly reflective copper surface then becomes an optical barrier to the absorption of the energy of subsequent layers. The process thus becomes self-limiting in terms of its ability to transfer energy to the material at substrate interface with a detrimental effect on the upper surface of the film or delamination of the film.

With the thicker film, the lower portions of the film act as a thermal sink taking that energy which is absorbed in the upper surface. If the energy taken does not raise the nano-copper beyond its sintering temperature, then there is no subsequent change in 
structure. The lower sintering visible at the substrate / ink interface will also likely have a significant effect on the contact resistance as intimate contact between the FTO, and a conductive copper cannot be guaranteed.

It was found that the photonically sintered features have a consistently higher resistance than the conventional oven sintered lines. Evidence for the change in sintering behaviour is clear from the images of the microstructure of the surface and within the film where the photonically processed film shows a lower level of interparticle sintering. Since some sintering is observed, this would suggest that the peak temperature required for sintering has been achieved. It is postulated that this level of sintering has some time-related element where the temperature has not been held for a sufficient amount of time for the sintering to complete. This hypothesis is reinforced when one examines Figure 4.3 and Table 4.1, where the near-identical levels of conductivity are achieved even though the energy level of the two lamp regimes is doubled. The pulse width of each is a similar case, and the net result is a similar result. This result suggests a mechanism of heating during the pulse, followed by rapid cooling at the end of the pulse and the time at maximum intensity is more of a control variable than the absolute amount of energy. More material would have allowed further validation of this hypothesis but may not have provided mechanistic conformation. Ultimately a model of the behaviour would benefit the process based on those which have been developed for thin-film silver photonic sintering [170].

The interaction between the line width and the degree of sintering has implication for circuit design and processing. The proposed explanation is that the thermal mass of the line alters the degree of sintering within the line structure (as explained in the previous paragraph). However, photonic uniformity over a larger area shows the maximum illumination area for the equipment used is $300 \mathrm{~mm} \times 150 \mathrm{~mm}$. Placement of the $50 \mathrm{~mm} \times 50 \mathrm{~mm}$ sample within this area such that edge effects are minimized was easily possible. Given that illumination edge effects are present and the sensitivity of the material to sintering conditions for larger areas envisaged within the thesis (500 $\mathrm{mm} \times 500 \mathrm{~mm}$ ) will require lamps dimensions which are beyond that currently available. 
The laser \& NIR techniques were found ineffective to sinter the copper ink examined in this thesis, severe damage caused into the copper film with a major ablation led to inferior adhesion. The damage is attributed to the high-power technique employed to sinter the copper ink.

\subsection{Silver blending}

The silver blending provided a positive impact on the electrical performance, which is beyond that predicted simply by its lower resistivity $\left(1.59 \mu \Omega \mathrm{cm}^{-1}\right.$ compared to 1.68 $\left.\mu \Omega \mathrm{cm}^{-1}\right)$. The silver therefore has a positive impact on creating the conductive structure and pathways through the film.

The introduction of silver as a blending material into the copper ink provides a realistic means of increasing the conductivity of the material while minimizing costs. Historically, copper has been around 150 times cheaper than silver [171], [172], [173] and is a driver for this thesis. Even if the particle manufacturing costs reduced this to a factor of 20 , the bill of materials by using a $25 \% \mathrm{Ag}, 75 \% \mathrm{Cu}$ blend instead of pure silver would reduce by a factor of 3.5. Even with fixed manufacturing and sales cost, there remains an opportunity for this material blend. This reduced material costs would have to be negated against the lower sintering temperatures that are generally needed for silver and the freedom to sinter in any environment 


\section{Chapter 8: Conclusion \& Future work}

An experimental study has been carried out on the sintering of thick film printing of copper inks to FTO coated glass substrates for a range of applications where printed tracks enhance the conductivity/functionality of the transparent surface. As a result of the study, the following can be stated.

1. The lowest resistances can be obtained using the pure nano copper ink with optimum performance being $1 / 3$ bulk resistivity. They also offer the advantage of a lower sintering temperature but a longer sintering time to achieve the lowest resistance. The superior electrical performance of the pure nano materials comes at the expense of poor adhesion performance.

2. Blending nano particles with micro particles leads to an increase in resistivity, typically $3-5$ times with higher sintering temperatures, but adhesion is greatly improved. There is an additional benefit of a wider temperature range where acceptable performance is achieved.

3. Thermal sintering provides a robust sintering method and there is a small penalty in sintering the micro/nano particle blend inks in an inert atmosphere instead of a reducing atmosphere. This provides a lower cost and safer manufacturing route.

4. There is a narrow operational window where broad spectrum photonic sintering can be used. Low lamp energies do not result in conductive structures while excessive lamp energy results in failure of the conductive structures by complete delamination. The optimum lamp energy is dependent on the size of the feature that is printed. These factors make photonic sintering challenging to adopt in any possible application.

5. It has not been possible to sinter the copper inks with near infra-red energy or laser. The laser produces an excessive localized energy absorption which causes failure in the printed film. The operational limits for NIR are limited in a similar manner to that seen with broad spectrum photonic sintering.

6. Blending copper nano-micro inks with silver nano particles can be advantageous in the way of maintaining the adhesion on FTO substrates while simultaneously enhancing the material conductivity. A $25 \%$ blend of nano- 
silver ink is optimum in terms of improving conductivity while also maintaining adhesion and limiting cost. This silver / copper blend provided the best overall balanced performance of any of the materials tested.

\section{1: Future work}

There are a number of elements which have been raised by this thesis where a lack of material supply limited the possible investigations, and these should be examined in further studies.

1. The work has focussed on thick film screen printing as low resistance was the primary goal. Still, there are other applications where absolute conductivity is not a primary consideration, so the examination of sintering technologies for thinner films should be investigated.

2. The thesis has demonstrated that there are an optimum time, temperature, and gaseous environment for sintering each nano / micro ink. The impact on this for economic production, particularly for larger substrate sizes, needs to be established.

3. There may be an opportunity for refining the operational window of the broadspectrum photonic sintering to fully establish whether the limits of operation are practical for a given application. Establishing models for the photonic sintering could also be enhanced through such an experimental study where the necessary physical / electrical capabilities do not exist.

4. There is scope for additional work in establishing whether a longer, but lower laser pulse technology could be used to successfully sinter a thick film on the FTO coated glass substrate. As with point 3 above, this could provide additional material constants which could be incorporated into numerical models of the laser sintering process.

5. There is an opportunity for expanding the understanding of the underlying fundamental mechanism by which the copper micro particles show greater adhesion to the FTO coated substrates compared to the nano particles. 


\section{Appendix A: Error Mitigation}

\begin{tabular}{|c|c|c|}
\hline Error & Impact & Mitigation \\
\hline $\begin{array}{l}\text { Screen printing process } \\
\text { varies }\end{array}$ & $\begin{array}{l}\text { Resistance will } \\
\text { change, Ideal sintering } \\
\text { conditions may vary }\end{array}$ & $\begin{array}{l}\text { Visual inspection of every } \\
\text { sample printed and comparison } \\
\text { with master }\end{array}$ \\
\hline $\begin{array}{l}\text { Oxidation during thermal } \\
\text { sintering }\end{array}$ & $\begin{array}{l}\text { - } \mathrm{CuO} \text { forms, } \\
\text { - } \quad \text { variability in data }\end{array}$ & $\begin{array}{l}\text { Oven flooded with } \mathrm{N}_{2}, \\
\mathrm{~N}_{2} \mathrm{H}_{2} \text { gas prior to thermal ramp }\end{array}$ \\
\hline Ink ages and changes & $\begin{array}{l}\text { Screen printing process } \\
\text { varies }\end{array}$ & Materials inspection \\
\hline $\begin{array}{l}\text { Substrate is } \\
\text { contaminated }\end{array}$ & $\begin{array}{l}\text { Poor adhesion, through } \\
\text { film / substrate } \\
\text { conductivity reduced }\end{array}$ & $\begin{array}{l}4 \text { stage cleaning } \\
\text { process adopted. }\end{array}$ \\
\hline $\begin{array}{l}\text { Damage from } \\
\text { measurement using } \\
\text { probes }\end{array}$ & $\begin{array}{l}\text { Poor adhesion, at the edge } \\
\text { of film }\end{array}$ & Use spring test probe \\
\hline $\begin{array}{l}\text { Sample changing due to } \\
\text { atmospheric exposure }\end{array}$ & Variability in data & $\begin{array}{l}\text { Samples sealed in } \\
\text { (kept in a vacuumed bag) }\end{array}$ \\
\hline Photonic Power varies & Variability in data & $\begin{array}{l}\text { Trial test, replacing the lamp if } \\
\text { necessary }\end{array}$ \\
\hline
\end{tabular}




\section{Chapter 9: Works Cited}

[1] W. G. Davenport, M. King, M. Schlesinger, and A. K. Biswas, "Costs of Copper Production,” Extr. Metall. Copp., pp. 385-399, 2002, doi: 10.1016/b978-0080440293/50026-5.

[2] V. A. Korada, V. K. R. R, K. Ps, and S. P. Singh, "Copper Conductive Inks: Synthesis and its Utilization in Flexible Electronics," RSC Adv., vol. 5, pp. 6398564030, 2015, doi: 10.1039/C5RA08205F.

[3] G. Di. M. R. Dabera, M. Walker, A. M. Sanchez, H. J. Pereira, R. Beanland, and R. A. Hatton, "Retarding oxidation of copper nanoparticles without electrical isolation and the size dependence of work function," Nat. Commun., vol. 8, no. 1, 2017, doi: 10.1038/s41467-017-01735-6.

[4] Y. Kim, B. Lee, S. Yang, I. Byun, I. Jeong, and S. M. Cho, "Use of copper ink for fabricating conductive electrodes and RFID antenna tags by screen printing," Curr. Appl. Phys., vol. 12, no. 2, pp. 473-478, 2012, doi: 10.1016/j.cap.2011.08.003.

[5] D. A. van den Ende, R. Hendriks, R. Cauchois, and W. A. Groen, "Large area photonic flash soldering of thin chips on flex foils for flexible electronic systems: In situ temperature measurements and thermal modelling," Electron. Mater. Lett., vol. 10, no. 6, pp. 1175-1183, 2014, doi: 10.1007/s13391-014-4222-3.

[6] S. Norita, D. Kumaki, Y. Kobayashi, T. Sato, K. Fukuda, and S. Tokito, "Inkjetprinted copper electrodes using photonic sintering and their application to organic thin-film transistors," Org. Electron., vol. 25, pp. 131-134, 2015, doi: 10.1016/j.orgel.2015.06.026.

[7] S.-H. Park, W.-H. Chung, and H.-S. Kim, “Temperature changes of copper nanoparticle ink during flash light sintering," J. Mater. Process. Technol., vol. 214, no. 11, pp. 2730-2738, 2014, doi: 10.1016/j.jmatprotec.2014.06.007. 
[8] A. Yabuki and S. Tanaka, "Electrically conductive copper film prepared at low temperature by thermal decomposition of copper amine complexes with various amines," Mater. Res. Bull., vol. 47, no. 12, pp. 4107-4111, 2012, doi: 10.1016/j.materresbull.2012.08.052.

[9] B. Lee, Y. Kim, S. Yang, I. Jeong, and J. Moon, "A low-cure-temperature copper nano ink for highly conductive printed electrodes," Curr. Appl. Phys., vol. 9, no. 2 SUPPL., pp. e157-e160, 2009, doi: 10.1016/j.cap.2009.03.008.

[10] W. H. Chung, H. J. Hwang, and H. S. Kim, "Flash light sintered copper precursor/nanoparticle pattern with high electrical conductivity and low porosity for printed electronics," Thin Solid Films, vol. 580, no. February 2016, pp. 61-70, 2015, doi: 10.1016/j.tsf.2015.03.004.

[11] S. Magdassi, M. Grouchko, and A. Kamyshny, "Copper nanoparticles for printed electronics: Routes towards achieving oxidation stability," Materials (Basel)., vol. 3, no. 9, pp. 4626-4638, 2010, doi: 10.3390/ma3094626.

[12] D. Deng, Y. Jin, Y. Cheng, T. Qi, and F. Xiao, “Copper Nanoparticles: Aqueous Phase Synthesis and Conductive Films Fabrication at Low Sintering Temperature," ACS Appl. Mater. Interfaces, vol. 5, no. 9, pp. 3839-3846, 2013, doi: 10.1021/am400480k

[13] C. Liu et al., "Synthesis and size control of nano/submicron copper particles by feeding strategies," 8th Annu. IEEE Int. Conf. Nano/Micro Eng. Mol. Syst. IEEE NEMS 2013, vol. 1, pp. 718-721, 2013, doi: 10.1109/NEMS.2013.6559829.

[14] K. Ida et al., "Behavior of $\mathrm{Cu}$ nanoparticles ink under reductive calcination for fabrication of $\mathrm{Cu}$ conductive film," Thin Solid Films, vol. 520, no. 7, pp. 2789-2793, 2012, doi: 10.1016/j.tsf.2011.12.024.

[15] P. F. M. De Oliveira, R. M. Torresi, F. Emmerling, and P. H. C. Camargo, 
"Challenges and opportunities in the bottom-up mechanochemical synthesis of noble metal nanoparticles," J. Mater. Chem. A, vol. 8, no. 32, pp. 16114-16141, 2020, doi: $10.1039 / \mathrm{d} 0 \mathrm{ta} 05183 \mathrm{~g}$.

[16] M. Joo, B. Lee, S. Jeong, and M. Lee, "Comparative studies on thermal and laser sintering for highly conductive $\mathrm{Cu}$ films printable on plastic substrate," Thin Solid Films, vol. 520, no. 7, pp. 2878-2883, 2012, doi: 10.1016/j.tsf.2011.11.078.

[17] H. J. Hwang, K. H. Oh, and H. S. Kim, "All-photonic drying and sintering process via flash white light combined with deep-UV and near-infrared irradiation for highly conductive copper nano-ink," Sci. Rep., vol. 6, no. May 2015, pp. 1-10, 2016, doi: 10.1038/srep19696.

[18] C.-H. Ryu, S.-J. Joo, and H.-S. Kim, “Two-step flash light sintering of copper nanoparticle ink to remove substrate warping," Appl. Surf. Sci., vol. 384, pp. 182191, 2016, doi: 10.1016/j.apsusc.2016.05.025.

[19] A. Koşar, "Effect of substrate thickness and material on heat transfer in microchannel heat sinks," Int. J. Therm. Sci., vol. 49, no. 4, pp. 635-642, 2010, doi: 10.1016/j.jithermalsci.2009.11.004.

[20] Y. Wang et al., "A facile process combined with inkjet printing, surface modification and electroless deposition to fabricate adhesion-enhanced copper patterns on flexible polymer substrates for functional flexible electronics," Electrochim. Acta, vol. 218, pp. 24-31, 2016, doi: 10.1016/j.electacta.2016.08.143.

[21] B. Abbas, Y. Mohammad, E. Jewell, and J. Searle, "Thermal sintering of printable copper for enhanced conductivity of FTO coated glass substrates," J. Mater. Sci. Mater. Electron., no. 0123456789, pp. 17-19, 2019, doi: 10.1007/s10854-01902358-x.

[22] F. De Rossi et al., "All Printable Perovskite Solar Modules with $198 \mathrm{~cm}^{2}$ Active 
Area and Over 6\% Efficiency," Adv. Mater. Technol., vol. 3, no. 11, p. 1800156 , Nov. 2018, doi: 10.1002/admt.201800156.

[23] Y. Galagan et al., "Evaluation of ink-jet printed current collecting grids and busbars for ITO-free organic solar cells,” Sol. Energy Mater. Sol. Cells, vol. 104, pp. 32-38, 2012, doi: 10.1016/j.solmat.2012.04.039.

[24] E. H. Jewell, S. M. Hamblyn, T. C. Claypole, and D. T. Gethin, "The impact of carbon content and mesh on the characteristics of screen printed conductive structures," Circuit World, vol. 39, no. 1, pp. 13-21, 2013, doi: 10.1108/03056121311298918.

[25] W. A. ANDERSON, W. A. Johnson, and F. N. RHINES, "Rates of high-temperature oxidation of dilute copper alloys," Aime Trans, vol. 147, no. 1368, pp. 205-221, 1942.

[26] E. Lamprecht, G. M. Watkins, and M. E. Brown, "The thermal decomposition of copper(II) oxalate revisited," Thermochim. Acta, vol. 446, no. 1-2, pp. 91-100, 2006, doi: 10.1016/j.tca.2006.03.008.

[27] K. Do Kim and H. T. Kim, "Comparison of the effect of reaction parameters on particle size in the formation of $\mathrm{SiO} 2, \mathrm{TiO} 2$, and $\mathrm{ZrO} 2$ nanoparticles," Mater. Lett., vol. 57, no. 21, pp. 3211-3216, 2003, doi: 10.1016/S0167-577X(03)00037-5.

[28] S. Yoda, Y. Takebayashi, K. Sue, T. Furuya, and K. Otake, "Thermal Decomposition of Copper (II) Acetylacetonate in Supercritical Carbon Dioxide: In situ Observation via UV-vis Spectroscopy,” J. Supercrit. Fluids, vol. 123, pp. 82-91, 2016, doi: 10.1016/j.supflu.2016.12.017.

[29] Z. Su et al., "Fabrication of Cu2ZnSnS4 solar cells with 5.1\% efficiency via thermal decomposition and reaction using a non-toxic sol-gel route," J. Mater. Chem. A, vol. 2, no. 2, pp. 500-509, 2014, doi: 10.1039/c3ta13533k. 
[30] M. Fukuda and N. Koga, "Kinetics and Mechanisms of the Thermal Decomposition of Copper(II) Hydroxide: A Consecutive Process Comprising Induction Period, Surface Reaction, and Phase Boundary-Controlled Reaction," J. Phys. Chem. C, vol. 122, no. 24, pp. 12869-12879, 2018, doi: 10.1021/acs.jpcc.8b03260.

[31] D. W. Bridges, J. P. Baur, G. S. Baur, and W. M. J. Fassell, "Oxidation of Copper to Cu2 O and CuO,” J. Electrochem. Soc., vol. 4, no. 1956, pp. 475-478, 1956, doi: 10.1149/1.2430387.

[32] S. Choudhary et al., "Oxidation mechanism of thin Cu films: A gateway towards the formation of single oxide phase," AIP Adv., vol. 8, no. 5, 2018, doi: 10.1063/1.5028407.

[33] S. Choudhary, J. V. N. Sarma, and S. Gangopadhyay, "Growth and characterization of single phase $\mathrm{Cu} 2 \mathrm{O}$ by thermal oxidation of thin copper films," AIP Conf. Proc., vol. 1724, no. April 2016, 2016, doi: 10.1063/1.4945236.

[34] M. Nolan and S. D. Elliott, "The p-type conduction mechanism in Cu2O: A first principles study," Phys. Chem. Chem. Phys., vol. 8, no. 45, pp. 5350-5358, 2006, doi: 10.1039/b611969g.

[35] Y. Zhu, K. Mimura, and M. Isshiki, “Oxidation Mechanism of Copper at 623-1073 K.," Mater. Trans., vol. 43, no. 9, pp. 2173-2176, 2002, doi: 10.2320/matertrans.43.2173

[36] S.-K. Lee, H.-C. Hsu, and W.-H. Tuan, "Oxidation Behavior of Copper at a Temperature below $300^{\circ} \mathrm{C}$ and the Methodology for Passivation," Mater. Res., vol. 19, no. 1, pp. 51-56, 2016, doi: 10.1590/1980-5373-MR-2015-0139.

[37] R. Haugsrud, "The Influence of Water Vapor on the Oxidation of Copper at Intermediate Temperatures," J. Electrochem. Soc., vol. 149, no. 1, pp. B14-B21, 2002, doi: 10.1149/1.1427076. 
[38] M. Yin et al., "Copper oxide nanocrystals," J. Am. Chem. Soc., vol. 127, no. 26, pp. 9506-9511, 2005, doi: 10.1021/ja050006u.

[39] E. Jewell, S. Hamblyn, T. Claypole, and D. Gethin, "Deposition of High Conductivity Low Silver Content Materials by Screen Printing," Coatings, vol. 5, no. 2, pp. 172-185, 2015, doi: 10.3390/coatings5020172.

[40] L. Meng, L. Shi, Y. Ge, J. Tang, Y. Chen, and H. Zhong, "Photon management of combining nanostructural antireflection and perovskite down-shifting composite films for improving the efficiency of silicon solar cells," Sol. Energy Mater. Sol. Cells, vol. 220, no. November 2020, p. 110856, 2021, doi: 10.1016/j.solmat.2020.110856.

[41] S. Cell, "Comparative Study of Perovskite and Silicon,” vol. III, no. Iv, pp. 16-19, 2014.

[42] M. Orrill, D. Abele, M. Wagner, and S. LeBlanc, "Ink synthesis and inkjet printing of electrostatically stabilized multilayer graphene nanoshells," J. Colloid Interface Sci., vol. 566, pp. 454-462, 2020, doi: 10.1016/j.jcis.2020.01.095.

[43] J. Hoornstra, A. W. Weeber, H. H. C. de Moor, and W. C. Sinke, "The importance of paste rheology in improving fine line, thick film screen printing of front side metallization," 14th Eur. Photovolt. Sol. energy Conf. Exhib., 1997, doi: 10.1016/0254-0584(96)80091-5.

[44] E. Khaled, G. G. Mohamed, and T. Awad, "Disposal screen-printed carbon paste electrodes for the potentiometric titration of surfactants," Sensors Actuators, $B$ Chem., vol. 135, no. 1, pp. 74-80, 2008, doi: 10.1016/j.snb.2008.07.027.

[45] L. Nayak, S. Mohanty, S. K. Nayak, and A. Ramadoss, "A review on inkjet printing of nanoparticle inks for flexible electronics," J. Mater. Chem. C, vol. 7, no. 29, pp. 8771-8795, 2019, doi: 10.1039/c9tc01630a. 
[46] F. Abbasi-Kesbi, A. M. Rashidi, and B. Astinchap, "Preparation of ultrafine grained copper nanoparticles via immersion deposit method," Appl. Nanosci., vol. 8, no. 3, pp. 221-230, 2018, doi: 10.1007/s13204-018-0646-7.

[47] R. Marković et al., "Copper-sulfate pentahydrate as a product of the waste sulfuric acid solution treatment," Metall. Mater. Trans. B Process Metall. Mater. Process. Sci., vol. 43, no. 6, pp. 1388-1392, 2012, doi: 10.1007/s11663-012-9721-8.

[48] F. J. Justel, D. M. Camacho, M. E. Taboada, and K. J. Roberts, "Crystallisation of copper sulphate pentahydrate from aqueous solution in absence and presence of sodium chloride," J. Cryst. Growth, vol. 525, no. May, p. 125204, 2019, doi: 10.1016/j.jcrysgro.2019.125204.

[49] M. Schmeißer, "Bachelor Thesis Reduction of Copper Oxide by Formic Acid," no. September, 2011.

[50] W. Li et al., "The rise of conductive copper inks: challenges and perspectives," Appl. Mater. Today, vol. 18, p. 100451, 2020, doi: 10.1016/j.apmt.2019.100451.

[51] M. Blosi, S. Albonetti, M. Dondi, C. Martelli, and G. Baldi, "Microwave-assisted polyol synthesis of Cu nanoparticles," J. Nanoparticle Res., vol. 13, no. 1, pp. 127138, 2011, doi: 10.1007/s11051-010-0010-7.

[52] Y. Zuo, J. Shen, H. Xu, and R. Gao, "Effect of different sizes of Cu nanoparticles on the shear strength of Cu-Cu joints," Mater. Lett., vol. 199, pp. 13-16, 2017, doi: 10.1016/j.matlet.2017.03.166.

[53] C. Cheng et al., "A novel method of synthesizing antioxidative copper nanoparticles for high performance conductive ink," J. Mater. Sci. Mater. Electron., vol. 28, no. 18, pp. 13556-13564, 2017, doi: 10.1007/s10854-017-7195-9.

[54] N. K. Roy, C. S. Foong, and M. A. Cullinan, "Effect of size, morphology, and synthesis method on the thermal and sintering properties of copper nanoparticles for 
use in microscale additive manufacturing processes," Addit. Manuf., vol. 21, no. September 2017, pp. 17-29, 2018, doi: 10.1016/j.addma.2018.02.008.

[55] J. P. Smith and W. W. Wendlandt, “COMPLEXES--XII,” vol. 26, no. Ii, 1964.

[56] T. M. D. Dang, T. T. T. Le, E. Fribourg-Blanc, and M. C. Dang, "Synthesis and optical properties of copper nanoparticles prepared by a chemical reduction method," Adv. Nat. Sci. Nanosci. Nanotechnol., vol. 2, no. 1, 2011, doi: 10.1088/2043$6262 / 2 / 1 / 015009$.

[57] S. Chandra, A. Kumar, and P. K. Tomar, "Synthesis and characterization of copper nanoparticles by reducing agent," J. Saudi Chem. Soc., vol. 18, no. 2, pp. 149-153, 2014, doi: 10.1016/j.jscs.2011.06.009.

[58] S. Jeong et al., "Controlling the thickness of the surface oxide layer on $\mathrm{Cu}$ nanoparticles for the fabrication of conductive structures by ink-jet printing," Adv. Funct. Mater., vol. 18, no. 5, pp. 679-686, 2008, doi: 10.1002/adfm.200700902.

[59] R. Baran, J. M. Krafft, T. Onfroy, T. Grzybek, and S. Dzwigaj, "Influence of the nature and environment of cobalt on the catalytic activity of Co-BEA zeolites in selective catalytic reduction of NO with ammonia," Microporous Mesoporous Mater., vol. 225, pp. 515-523, 2016, doi: 10.1016/j.micromeso.2015.12.061.

[60] H. V. Tartar and W. L. Semon, "The reaction between copper and nitrogen peroxide," J. Am. Chem. Soc., vol. 43, no. 3, pp. 494-500, 1921, doi: $10.1021 / \mathrm{ja} 01436 \mathrm{a} 012$.

[61] D. Ji et al., "Nitrogen-doped graphene wrapped around silver nanowires for enhanced catalysis in oxygen reduction reaction," J. Solid State Electrochem., vol. 22, no. 7, pp. 2287-2296, 2018, doi: 10.1007/s10008-018-3914-2.

[62] I. Kim and J. Kim, "The effect of reduction atmospheres on the sintering behaviors of inkjet-printed Cu interconnectors," J. Appl. Phys., vol. 108, no. 10, 2010, doi: 
10.1063/1.3511688.

[63] Y. Hokita, M. Kanzaki, T. Sugiyama, R. Arakawa, and H. Kawasaki, "HighConcentration Synthesis of Sub-10-nm Copper Nanoparticles for Application to Conductive Nanoinks," ACS Appl. Mater. Interfaces, vol. 7, no. 34, pp. 19382 19389, 2015, doi: 10.1021/acsami.5b05542.

[64] T. Yonezawa, H. Tsukamoto, Y. Yong, M. T. Nguyen, and M. Matsubara, "Low temperature sintering process of copper fine particles under nitrogen gas flow with

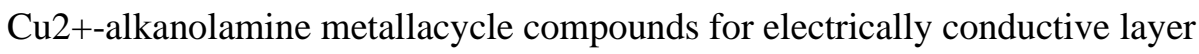
formation," RSC Adv., vol. 6, no. 15, pp. 12048-12052, 2016, doi: $10.1039 / \mathrm{c} 5 \mathrm{ra} 25058 \mathrm{~g}$.

[65] Y. Kamikoriyama, H. Imamura, A. Muramatsu, and K. Kanie, “Ambient AqueousPhase Synthesis of Copper Nanoparticles and Nanopastes with Low-Temperature Sintering and Ultra-High Bonding Abilities," Sci. Rep., vol. 9, no. 1, pp. 1-10, 2019, doi: 10.1038/s41598-018-38422-5.

[66] A. Yabuki and N. Arriffin, "Electrical conductivity AR of copper nanoparticle thin films annealed at low temperature," Thin Solid Films, vol. 518, no. 23, pp. 70337037, 2010, doi: 10.1016/j.tsf.2010.07.023.

[67] M. Schneegans and M. Heinrici, “(12) Patent Application Publication (10) Pub. No.: US 2015/0214095 A1,” vol. 1, no. 19, 2015.

[68] J. Liu, H. Ji, S. Wang, and M. Li, “The low temperature exothermic sintering of formic acid treated $\mathrm{Cu}$ nanoparticles for conductive ink," J. Mater. Sci. Mater. Electron., 2016, doi: 10.1007/s10854-016-5476-3.

[69] W. Yang, X. Li, X. Du, Y. Deng, and H. Dai, "Effective low-temperature hydrogenolysis of lignin using carbon-supported ruthenium and formic acid as reducing agent," Catal. Commun., vol. 126, no. February, pp. 30-34, 2019, doi: 
10.1016/j.catcom.2019.04.025.

[70] Y. Farraj, M. Grouchko, and S. Magdassi, "Self-reduction of a copper complex MOD ink for inkjet printing conductive patterns on plastics," Chem. Commun., vol. 51, no. 9, pp. 1587-1590, 2015, doi: 10.1039/c4cc08749f.

[71] F. Hermerschmidt et al., "Truly Low Temperature Sintering of Printed Copper Ink Using Formic Acid," Adv. Mater. Technol., vol. 3, no. 12, pp. 1-6, 2018, doi: 10.1002/admt.201800146.

[72] Y. Lee, J.-R. Choi, K. J. Lee, N. E. Stott, and D. Kim, "Large-scale synthesis of copper nanoparticles by chemically controlled reduction for applications of inkjetprinted electronics," Nanotechnology, vol. 19, no. 41, p. 415604, 2008, doi: 10.1088/0957-4484/19/41/415604.

[73] S. J. Kim, J. Lee, Y. H. Choi, D. H. Yeon, and Y. Byun, "Effect of copper concentration in printable copper inks on film fabrication," Thin Solid Films, vol. 520, no. 7, pp. 2731-2734, 2012, doi: 10.1016/j.tsf.2011.11.056.

[74] G. Egels, R. Fussik, S. Weber, and W. Theisen, "On the role of nitrogen on hydrogen environment embrittlement of high-interstitial austenitic $\mathrm{CrMnC}(\mathrm{N})$ steels," Int. J. Hydrogen Energy, vol. 44, no. 60, pp. 32323-32331, 2019, doi: 10.1016/j.ijhydene.2019.10.109.

[75] D. Ding, H. Peng, W. Peng, Y. Yu, G. Wu, and J. Zhang, "Isothermal hydrogen reduction of oxide scale on hot-rolled steel strip in 30 pct H2-N2 atmosphere," Int. J. Hydrogen Energy, vol. 42, no. 50, pp. 29921-29928, 2017, doi: 10.1016/j.ijhydene.2017.07.020.

[76] J. Liu, H. Chen, H. Ji, and M. Li, "Highly Conductive $\mathrm{Cu}-\mathrm{Cu}$ Joint Formation by Low-Temperature Sintering of Formic Acid-Treated Cu Nanoparticles,” 2016, doi: 10.1021/acsami.6b10280. 
[77] K. A. Schroder, S. C. Mccool, and W. F. Furlan, "Broadcast Photonic Curing of Metallic Nanoparticle Films Basic Process Research and Development System,” Nanotechnol. Conf. Trade Show, vol. 3, no. 512, pp. 198-201, 2006.

[78] D. Lopez-Espiricueta, E. Fearon, S. Edwardson, and G. Dearden, "Direct write processing of multi-micron thickness copper nano-particle paste on flexible substrates with 532 nm laser wavelength," Phys. Procedia, vol. 83, pp. 194-203, 2016, doi: 10.1016/j.phpro.2016.08.008.

[79] M. H. Yu, S. J. Joo, and H. S. Kim, "Multi-pulse flash light sintering of bimodal $\mathrm{Cu}$ nanoparticle-ink for highly conductive printed Cu electrodes," Nanotechnology, vol. 28, no. 20, 2017, doi: 10.1088/1361-6528/aa6cda.

[80] S. Das, D. Cormier, and S. Williams, "Potential for Multi-functional Additive Manufacturing Using Pulsed Photonic Sintering," Procedia Manuf., vol. 1, pp. 366377, 2015, doi: 10.1016/j.promfg.2015.09.043.

[81] M. H. Yu, S. J. Joo, and H. S. Kim, "Multi-pulse flash light sintering of bimodal Cu nanoparticle-ink for highly conductive printed Cu electrodes," Nanotechnology, vol. 28, no. 20, 2017, doi: 10.1088/1361-6528/aa6cda.

[82] W.-S. Han, J.-M. Hong, H.-S. Kim, and Y.-W. Song, "Multi-pulsed white light sintering of printed Cu nanoinks.," Nanotechnology, vol. 22, no. 39, p. 395705, 2011, doi: 10.1088/0957-4484/22/39/395705.

[83] S. R. Dhage and H. T. Hahn, "Rapid treatment of CIGS particles by intense pulsed light," J. Phys. Chem. Solids, vol. 71, no. 10, pp. 1480-1483, 2010, doi: 10.1016/j.jpcs.2010.07.016.

[84] T. Echániz, R. B. Pérez-Sáez, and M. J. Tello, “Optical properties of metals: Infrared emissivity in the anomalous skin effect spectral region," J. Appl. Phys., vol. 116, no. 9, 2014, doi: 10.1063/1.4894169. 
[85] H. Ennaceri, D. Barrit, A. Khaldoun, A. Benyoussef, and A. Ennaoui, "Deposition of transparent Aluminum Oxide (A12O3) films on silvered CSP mirrors,” Proc. 2014 Int. Renew. Sustain. Energy Conf. IRSEC 2014, no. 1, pp. 114-119, 2014, doi: 10.1109/IRSEC.2014.7059915.

[86] A. V. Kalenskii, A. A. Zvekov, A. P. Nikitin, and M. V. Anan'eva, “Optical Properties of Copper Nanoparticles," Russ. Phys. J., vol. 58, no. 8, pp. 1098-1104, 2015, doi: 10.1007/s11182-015-0618-2.

[87] Z. N. Kayani and H. Aslam, "Investigation of structural, optical, antibacterial, and dielectric properties of V-doped copper oxide thin films: Comparison with undoped copper oxide thin films," Adv. Powder Technol., vol. 32, no. 7, pp. 2345-2358, 2021, doi: 10.1016/j.apt.2021.05.026.

[88] E.-B. Jeon, S.-J. Joo, H. Ahn, and H.-S. Kim, “Two-step flash light sintering process for enhanced adhesion between copper complex ion/silane ink and a flexible substrate," Thin Solid Films, vol. 603, pp. 382-390, 2016, doi: 10.1016/j.tsf.2016.02.033.

[89] C. H. Ryu, S. J. Joo, and H. S. Kim, "Two-step flash light sintering of copper nanoparticle ink to remove substrate warping," Appl. Surf. Sci., vol. 384, pp. 182191, 2016, doi: 10.1016/j.apsusc.2016.05.025.

[90] D. Salamon, Flash Sintering and Other Rapid Sintering Techniques. Elsevier Ltd., 2020.

[91] E. Halonen, E. Heinonen, and M. Mäntysalo, "The Effect of Laser Sintering Process Parameters on Cu Nanoparticle Ink in Room Conditions," Opt. Photonics J., vol. 03, no. 04, pp. 40-44, 2013, doi: 10.4236/opj.2013.34A007.

[92] M. D. Bilandžić, S. Wollgarten, J. Stollenwerk, R. Poprawe, M. Esteves-Oliveira, and H. Fischer, "Glass-ceramic coating material for the $\mathrm{CO} 2$ laser based sintering of 
thin films as caries and erosion protection," Dent. Mater., vol. 33, no. 9, pp. 9951003, 2017, doi: 10.1016/j.dental.2017.06.008.

[93] J. Živčák, M. Šarik, and R. Hudák, "FEA simulation of thermal processes during the direct metal laser sintering of Ti64 titanium powder," Meas. J. Int. Meas. Confed., vol. 94, pp. 893-901, 2016, doi: 10.1016/j.measurement.2016.07.072.

[94] D. Drummer, S. Greiner, M. Zhao, and K. Wudy, "A novel approach for understanding laser sintering of polymers," Addit. Manuf., vol. 27, no. March, pp. 379-388, 2019, doi: 10.1016/j.addma.2019.03.012.

[95] Y. Yang, Z. Li, S. Yang, Y. Li, and J. Huang, "Multiscale simulation study of laser sintering of inkjet-printed silver nanoparticle inks," Int. J. Heat Mass Transf., vol. 159, p. 120110, 2020, doi: 10.1016/j.ijheatmasstransfer.2020.120110.

[96] O. Koritsoglou et al., "Copper micro-electrode fabrication using laser printing and laser sintering processes for on-chip antennas on flexible integrated circuits," Opt. Mater. Express, vol. 9, no. 7, p. 3046, 2019, doi: 10.1364/ome.9.003046.

[97] J. Kwon et al., "Low-Temperature Oxidation-Free Selective Laser Sintering of Cu Nanoparticle Paste on a Polymer Substrate for the Flexible Touch Panel Applications," ACS Appl. Mater. Interfaces, vol. 8, no. 18, pp. 11575-11582, 2016, doi: $10.1021 /$ acsami.5b12714.

[98] M. Joo, B. Lee, S. Jeong, and M. Lee, "Laser sintering of Cu paste film printed on polyimide substrate," Appl. Surf. Sci., vol. 258, no. 1, pp. 521-524, 2011, doi: 10.1016/j.apsusc.2011.08.076.

[99] M. Zenou, O. Ermak, A. Saar, and Z. Kotler, "Laser sintering of copper nanoparticles," J. Phys. D. Appl. Phys., vol. 47, no. 2, p. 025501, 2014, doi: 10.1088/0022-3727/47/2/025501.

[100] C. W. Cheng and J. K. Chen, "Femtosecond laser sintering of copper nanoparticles," 
Appl. Phys. A, vol. 122, no. 4, p. 289, 2016, doi: 10.1007/s00339-016-9814-3.

[101] J. Her, D. Cho, and C. S. Lee, "Synthesis of Conductive Nano Ink Using 1Octanethiol Coated Copper Nano Powders in 1-Octanol for Low Temperature Sintering Process," Mater. Trans., vol. 54, no. 4, pp. 599-602, 2013, doi: 10.2320/matertrans.M2012363.

[102] S. Hong et al., "Rapid surface kinetics enhancement via flash light sintering for lowtemperature solid oxide fuel cells," J. Alloys Compd., vol. 778, pp. 337-344, 2019, doi: 10.1016/j.jallcom.2018.11.178.

[103] B. G. Park, C. J. Lee, and S. B. Jung, "Enhancing adhesion strength of photonic sintered screen-printed Ag circuit by atmospheric pressure plasma," Microelectron. Eng., vol. 202, no. September, pp. 37-41, 2018, doi: 10.1016/j.mee.2018.10.006.

[104] N. M. Twyman, K. Tetzner, T. D. Anthopoulos, D. J. Payne, and A. Regoutz, "Rapid photonic curing of solution-processed In 2 O 3 layers on flexible substrates," Appl. Surf. Sci., vol. 479, no. November 2018, pp. 974-979, 2019, doi: 10.1016/j.apsusc.2019.02.038.

[105] E. Drahi, S. Blayac, A. Borbely, and P. Benaben, "Impact of ink synthesis on processing of inkjet-printed silicon nanoparticle thin films: A comparison of Rapid Thermal Annealing and photonic sintering,” Thin Solid Films, vol. 574, pp. 169-176, 2015, doi: 10.1016/j.tsf.2014.11.079.

[106] R. Alias and S. Shapee, "Rheological Behaviors and Their Correlation with Printing Performance of Silver Paste for LTCC Tape," Cdn.Intechopen.Com, 2010, doi: $10.5772 / 35004$.

[107] B. Abbas, "Examination of the effect of ultrasound on electroless copper plating Examination of the effect of ultrasound on electroless copper plating A thesis submitted in partial fulfilment of the University 's requirements," Coventry Univ., 
no. October, 2013.

[108] A. J. Cobley, I. Tudela, B. Abbas, and B. Mkhlef, "Low-frequency 'delay time' ultrasound and its effect on electroless $\mathrm{Cu}$ metallisation of a Pd activated dielectric material," Thin Solid Films, vol. 597, pp. 226-230, 2015, doi:

10.1016/j.tsf.2015.11.068.

[109] R. Dharmadasa, M. Jha, D. a Amos, and T. Druffel, "Room temperature synthesis of a copper ink for the intense pulsed light sintering of conductive copper films.," ACS Appl. Mater. Interfaces, vol. 5, pp. 13227-34, 2013, doi: 10.1021/am404226e.

[110] F. Meng, J. Huang, T. Cao, P. Zhao, and P. Li, "Multi-sensor feedback control for the flash sintering of printed nano-silver ink," J. Manuf. Process., vol. 53, no. January, pp. 356-363, 2020, doi: 10.1016/j.jmapro.2020.02.025.

[111] F. Maccarrone and G. Paffuti, "Capacitance and forces for two square electrodes," $J$. Electrostat., vol. 89, pp. 20-29, 2017, doi: 10.1016/j.elstat.2017.06.007.

[112] P. Dianat, Unconventional photo capacitor with giant light induced capacitance enhancement. Elsevier Ltd, 2015.

[113] W. H. Chung, H. J. Hwang, S. H. Lee, and H. S. Kim, "In situ monitoring of a flash light sintering process using silver nano-ink for producing flexible electronics," Nanotechnology, vol. 24, no. 3, 2013, doi: 10.1088/0957-4484/24/3/035202.

[114] L. Amplification, "Aside from the energetically loaded laser-active medium - the crystal, liquid or gas - it is necessary to feed back the generated photons into the medium us- ing a," Science (80-. )., 1960.

[115] K. Kuroda, H. Nakako, M. Inada, T. Noudou, and Y. Kumashiro, "Development of Copper Materials and Processing for Printed Electronics," vol. 5, no. 1, pp. 20-25, 2012.

[116] J. Venegas, "PROBLEM SET 5," 2004. 
[117] S. Loganathan, R. B. Valapa, R. Mishra, and G. Pugazhenthi, Copyright, no. March 2018. 2017.

[118] C. Branca and C. Di Blasi, "Self-heating effects in the thermogravimetric analysis of wood char oxidation," Fuel, vol. 276, no. April 2019, p. 118012, 2020, doi: 10.1016/j.fuel.2020.118012.

[119] L. Soares and A. Alves, "Analysis of colorimetry using the CIE-L*a*b* system and the photocatalytic activity of photochromic films," Mater. Res. Bull., vol. 105, no. January, pp. 318-321, 2018, doi: 10.1016/j.materresbull.2018.05.012.

[120] A. Mansourian, H. Saidpour, and S. H. Mannan, "Conductive pattern, electrical and mechanical characterisation of gold nanorods inside porous alumina template," Appl. Surf. Sci., vol. 532, no. June, p. 147426, 2020, doi: 10.1016/j.apsusc.2020.147426.

[121] M. Fang, D. Li, H. Lin, C. Luo, R. Qi, and H. Peng, "Flexible and recyclable conductive composite based on few-layered graphene with excellent self-healing capability," Eur. Polym. J., vol. 108, no. September, pp. 536-541, 2018, doi: 10.1016/j.eurpolymj.2018.09.005.

[122] S. Son, S. Lee, and J. Choi, "Fine metal line patterning on hydrophilic nonconductive substrates based on electrohydrodynamic printing and laser sintering," $J$. Electrostat., vol. 72, no. 1, pp. 70-75, 2014, doi: 10.1016/j.elstat.2013.12.002.

[123] S. Grover, "Effect of Transmission Line Measurement ( TLM ) Geometry on Specific Contact Resistivity Determination Effect of Transmission Line Measurement," p. $112,2016$.

[124] F. M. Smits, "Measurement the of Sheet Resistivities Four-Point Probe," pp. 711$718,1957$.

[125] W. Han, H. Jiao, and D. Fox, Scanning electron microscopy, vol. 272. Tsinghua University Press Limited, 2018. 
[126] M. de Assumpção Pereira-da-Silva and F. A. Ferri, 1 - Scanning Electron Microscopy. Elsevier Inc., 2017.

[127] M. Joshi, A. Bhattacharyya, and S. W. Ali, "Characterization techniques for nanotechnology applications in textiles," Indian J. Fibre Text. Res., vol. 33, no. 3, pp. 304-317, 2008.

[128] D. D. NGUYEN, J.-H. TORTAI, M. ABAIDI, and P. SCHIAVONE, “FEM simulation of charging effect during SEM metrology," 2018, p. 42, doi: $10.1117 / 12.2326609$.

[129] J. M. Bijvoet, J. D. Bernal, and A. L. Patterson, "Forty Years of X-Ray diffraction," Nature, vol. 169, no. 4310, pp. 949-950, 1952, doi: 10.1038/169949a0.

[130] G. M. Hansford, "X-ray diffraction without sample preparation: Proof-of-principle experiments," Nucl. Instruments Methods Phys. Res. Sect. A Accel. Spectrometers, Detect. Assoc. Equip., vol. 728, pp. 102-106, 2013, doi: 10.1016/j.nima.2013.06.065.

[131] H. Nozaki, H. Matsuo, Y. Hayashi, H. Kadoura, and S. Fujita, "Microstructure of a solid oxide fuel cell by microbeam X-ray diffraction," Mater. Lett., vol. 277, p. 128383, 2020, doi: 10.1016/j.matlet.2020.128383.

[132] D. S. Mast et al., "Equation of state for technetium from X-ray diffraction and firstprinciple calculations," J. Phys. Chem. Solids, vol. 95, pp. 6-11, 2016, doi: 10.1016/j.jpcs.2016.03.007.

[133] E. H. Jewell, S. M. Hamblyn, T. C. Claypole, and D. T. Gethin, "The impact of carbon content and mesh on the characteristics of screen printed conductive structures," Circuit World, vol. 39, no. 1, pp. 13-21, Feb. 2013, doi: $10.1108 / 03056121311298918$.

[134] H. Lin, C. Chang, W. Hwu, and M. Ger, "The rheological behaviors of screenprinting pastes," J. Mater. Process. Technol., vol. 197, no. 1-3, pp. 284-291, Feb. 
2008, doi: 10.1016/j.jmatprotec.2007.06.067.

[135] T. Kant et al., "Flexible printed paper electrode with silver nano-ink for electrochemical applications," Microchem. J., vol. 155, no. November 2019, p. 104687, 2020, doi: 10.1016/j.microc.2020.104687.

[136] S. Y. Lee et al., "The development and investigation of highly stretchable conductive inks for 3-dimensional printed in-mold electronics," Org. Electron., vol. 85, no. June, p. 105881, 2020, doi: 10.1016/j.orgel.2020.105881.

[137] H. Y. Jun, E. J. Lee, and S. O. Ryu, "Synthesis and characterization of copper ink and direct printing of copper patterns by inkjet printing for electronic devices," Curr. Appl. Phys., vol. 20, no. 7, pp. 853-861, 2020, doi: 10.1016/j.cap.2020.04.003.

[138] X. F. Tang, Z. G. Yang, and W. J. Wang, “A simple way of preparing highconcentration and high-purity nano copper colloid for conductive ink in inkjet printing technology," Colloids Surfaces A Physicochem. Eng. Asp., vol. 360, no. 1-3, pp. 99-104, 2010, doi: 10.1016/j.colsurfa.2010.02.011.

[139] V. I. Levitas, "Mechanochemical mechanism for reaction of aluminium particles Subject Areas : Author for correspondence :," 2013.

[140] A. Kumar, A. Saxena, A. De, R. Shankar, and S. Mozumdar, "Facile synthesis of size-tunable copper and copper oxide nanoparticles using reverse microemulsions," RSC Adv., vol. 3, no. 15, pp. 5015-5021, 2013, doi: 10.1039/c3ra23455j.

[141] Y. Zhu, K. Mimura, J. W. Lim, M. Isshiki, and Q. Jiang, "Brief review of oxidation kinetics of copper at $350{ }^{\circ} \mathrm{C}$ to $1050{ }^{\circ} \mathrm{C}$," Metall. Mater. Trans. A Phys. Metall. Mater. Sci., vol. 37, no. 4, pp. 1231-1237, 2006, doi: 10.1007/s11661-006-1074-y.

[142] C. D. S. Tuck, C. A. Powell, and J. Nuttall, "Corrosion of copper and its alloys," Shreir's Corros., pp. 1937-1973, 2010, doi: 10.1016/B978-044452787-5.00094-9.

[143] A. Salc1 and R. Solmaz, "Fabrication of rhodanine self-assembled monolayer thin 
films on copper: Solvent optimization and corrosion inhibition studies," Prog. Org. Coatings, vol. 125, no. September, pp. 516-524, 2018, doi:

10.1016/j.porgcoat.2018.09.020.

[144] D. Mott, J. Galkowski, L. Wang, J. Luo, and C. J. Zhong, "Synthesis of sizecontrolled and shaped copper nanoparticles," Langmuir, vol. 23, no. 10, pp. 57405745, 2007, doi: 10.1021/la0635092.

[145] Z. Huang et al., "3D printing of ceramics and graphene circuits-on-ceramics by thermal bubble inkjet technology and high temperature sintering," Ceram. Int., vol. 46, no. 8, pp. 10096-10104, 2020, doi: 10.1016/j.ceramint.2019.12.278.

[146] X. Wu, S. Shao, Z. Chen, and Z. Cui, "Printed highly conductive Cu fi lms with strong adhesion enabled by low-energy photonic sintering on low-Tg fl exible plastic substrate."

[147] C. L. Lee, K. C. Chang, and C. M. Syu, "Silver nanoplates as inkjet ink particles for metallization at a low baking temperature of $100^{\circ} \mathrm{C}$," Colloids Surfaces A Physicochem. Eng. Asp., vol. 381, no. 1-3, pp. 85-91, 2011, doi: 10.1016/j.colsurfa.2011.03.034.

[148] J. Y. Kim, J. A. Rodriguez, J. C. Hanson, A. I. Frenkel, and P. L. Lee, "Reduction of $\mathrm{CuO}$ and $\mathrm{Cu} 2 \mathrm{O}$ with $\mathrm{H} 2: \mathrm{H}$ embedding and kinetic effects in the formation of suboxides," J. Am. Chem. Soc., vol. 125, no. 35, pp. 10684-10692, 2003, doi: 10.1021/ja0301673.

[149] M. G. Helander, M. T. Greiner, Z. B. Wang, W. M. Tang, and Z. H. Lu, "Work function of fluorine doped tin oxide," J. Vac. Sci. Technol. A Vacuum, Surfaces, Film., vol. 29, no. 1, p. 011019, 2011, doi: 10.1116/1.3525641.

[150] P. R. Huang, Y. He, C. Cao, and Z. H. Lu, "The origin of the high work function of chlorinated indium tin oxide," NPG Asia Mater., vol. 5, no. 8, pp. 1-5, 2013, doi: 
10.1038/am.2013.33.

[151] Y. H. Son, J. Y. Jang, M. K. Kang, S. Ahn, and C. S. Lee, “Application of flash-light sintering method to flexible inkjet printing using anti-oxidant copper nanoparticles," Thin Solid Films, vol. 656, no. September 2017, pp. 61-67, 2018, doi: 10.1016/j.tsf.2018.04.034.

[152] S.-J. Joo, H.-J. Hwang, and H.-S. Kim, "Highly conductive copper nano/microparticles ink via flash light sintering for printed electronics.," Nanotechnology, vol. 25, no. 26, p. 265601, 2014, doi: 10.1088/0957$4484 / 25 / 26 / 265601$.

[153] J. M. Home-Ortiz, R. Vargas, L. H. Macedo, and R. Romero, "Joint reconfiguration of feeders and allocation of capacitor banks in radial distribution systems considering voltage-dependent models," Int. J. Electr. Power Energy Syst., vol. 107, no. July 2018, pp. 298-310, 2019, doi: 10.1016/j.ijepes.2018.11.035.

[154] L. R. de Araujo, D. R. R. Penido, S. Carneiro, and J. L. R. Pereira, “Optimal unbalanced capacitor placement in distribution systems for voltage control and energy losses minimization,” Electr. Power Syst. Res., vol. 154, pp. 110-121, 2018, doi: 10.1016/j.epsr.2017.08.012.

[155] Y. S. Rosen, A. Yakushenko, A. Offenhäusser, and S. Magdassi, "Self-Reducing Copper Precursor Inks and Photonic Additive Yield Conductive Patterns under Intense Pulsed Light,” ACS Omega, vol. 2, no. 2, pp. 573-581, 2017, doi: 10.1021/acsomega.6b00478.

[156] J. W. Youn, S. J. Lee, K. S. Kim, and D. U. Kim, "Adhesion characteristics of VO 2 ink film sintered by intense pulsed light for smart window," Appl. Surf. Sci., vol. 441, pp. 508-514, 2018, doi: 10.1016/j.apsusc.2018.02.061.

[157] J. Troughton et al., "Photonic flash-annealing of lead halide perovskite solar cells in 
1 ms," J. Mater. Chem. A, vol. 4, no. 9, pp. 3471-3476, 2016, doi:

$10.1039 / \mathrm{c} 5 \mathrm{ta} 09431 \mathrm{c}$.

[158] H. Hannebauer, T. Dullweber, T. Falcon, X. Chen, and R. Brendel, "Record low ag paste consumption of $67.7 \mathrm{mg}$ with dual print," Energy Procedia, vol. 43, no. April, pp. 66-71, 2013, doi: 10.1016/j.egypro.2013.11.089.

[159] E. Seok et al., "A Comparative Study of Two Different SnO 2: F-coated Glass Substrates for CdTe Solar Cells," vol. 5, no. 1, pp. 1-8, 2017.

[160] K. Hooper, M. Carnie, C. Charbonneau, and T. Watson, "Near infrared radiation as a rapid heating technique for TiO2 films on glass mounted dye-sensitized solar cells," Int. J. Photoenergy, vol. 2014, no. 1, 2014, doi: 10.1155/2014/953623.

[161] J. V. Oboňa et al., "Surface melting of copper by ultrashort laser pulses," WIT Trans. Eng. Sci., vol. 71, pp. 171-179, 2011, doi: 10.2495/SECM110151.

[162] M. S. Rafique et al., "XRD and SEM analysis of a laser-irradiated cadmium," Laser Phys., vol. 17, no. 9, pp. 1138-1145, 2007, doi: 10.1134/s1054660x0709006x.

[163] J. Hermann, S. Noël, T. E. Itina, E. Axente, and M. E. Povarnitsyn, “Correlation between ablation efficiency and nanoparticle generation during the short-pulse laser ablation of metals," Laser Phys., vol. 18, no. 4, pp. 374-379, 2008, doi: $10.1134 / \mathrm{s} 11490-008-4002-6$.

[164] H. Jeong, J. Y. Kim, B. Koo, H. J. Son, D. Kim, and M. J. Ko, "Rapid sintering of MoS2 counter electrode using near-infrared pulsed laser for use in highly efficient dye-sensitized solar cells," J. Power Sources, vol. 330, pp. 104-110, 2016, doi: 10.1016/j.jpowsour.2016.09.002.

[165] Y. Wu et al., "Near-infrared long-persistent phosphor of Zn3Ga2Ge2O10: Cr3+ sintered in different atmosphere," Spectrochim. Acta - Part A Mol. Biomol. Spectrosc., vol. 151, pp. 385-389, 2015, doi: 10.1016/j.saa.2015.06.117. 
[166] F. Gaspar, J. Lopes, H. Cruz, M. Schwanninger, and J. Rodrigues, “Application of near infrared spectroscopy and multivariate data analysis for the evaluation of glue lines of untreated and copper azole treated laminated timber before and after ageing," Polym. Degrad. Stab., vol. 94, no. 7, pp. 1061-1071, 2009, doi: 10.1016/j.polymdegradstab.2009.04.011.

[167] A. H. Hamad, "Effects of Different Laser Pulse Regimes (Nanosecond, Picosecond and Femtosecond) on the Ablation of Materials for Production of Nanoparticles in Liquid Solution," High Energy Short Pulse Lasers, 2016, doi: 10.5772/63892.

[168] M. Grouchko, A. Kamyshny, and S. Magdassi, "Formation of air-stable copper-silver core-shell nanoparticles for inkjet printing," J. Mater. Chem., vol. 19, no. 19, pp. 3057-3062, 2009, doi: 10.1039/b821327e.

[169] K. H. Jung, J. Kim, B. G. Park, C. J. Lee, H. J. Sung, and S. B. Jung, "Fabrication of Ag circuit embedded in PDMS substrate and its mechanical and electrical property with variations of photonic energy," J. Alloys Compd., vol. 748, pp. 898-904, 2018, doi: 10.1016/j.jallcom.2018.03.171.

[170] L. Mo et al., "Silver nanoparticles based ink with moderate sintering in flexible and printed electronics," Int. J. Mol. Sci., vol. 20, no. 9, 2019, doi: 10.3390/ijms20092124.

[171] M. Singh and S. Rana, "Silver and copper nanowire films as cost-effective and robust transparent electrode in energy harvesting through photovoltaic: A review," Mater. Today Commun., vol. 24, no. June, 2020, doi: 10.1016/j.mtcomm.2020.101317.

[172] M. Jacoby, “The future of solar cells?," Chem. Eng. News, vol. 94, no. 18, pp. 30-35, 2016, doi: 10.1016/s1369-7021(04)00087-2. 
Chapter 9

[173] M. Cai, Y. Wu, H. Chen, X. Yang, Y. Qiang, and L. Han, “Cost-Performance Analysis of Perovskite Solar Modules," Adv. Sci., vol. 4, no. 1, 2017, doi: 10.1002/advs.201600269. 